\title{
A SEARCH FOR STARS OF VERY LOW METAL ABUNDANCE. VI. DETAILED ABUNDANCES OF 313 METAL-POOR STARS*
}

\author{
Ian U. Roederer ${ }^{1,3}$, George W. Preston ${ }^{1}$, Ian B. Thompson ${ }^{1}$, Stephen A. Shectman ${ }^{1}$, \\ Christopher Sneden ${ }^{2}$, Gregory S. Burley ${ }^{1}$, And Daniel D. Kelson ${ }^{1}$ \\ ${ }^{1}$ Carnegie Observatories, 813 Santa Barbara Street, Pasadena, CA 91101, USA; iur@ umich.edu \\ ${ }^{2}$ Department of Astronomy, University of Texas at Austin, 1 University Station, C1400, Austin, TX 78712, USA \\ Received 2013 August 20; accepted 2014 March 7; published 2014 May 2
}

\begin{abstract}
We present radial velocities, equivalent widths, model atmosphere parameters, and abundances or upper limits for 53 species of 48 elements derived from high resolution optical spectroscopy of 313 metal-poor stars. A majority of these stars were selected from the metal-poor candidates of the HK Survey of Beers, Preston, and Shectman. We derive detailed abundances for $61 \%$ of these stars for the first time. Spectra were obtained during a $10 \mathrm{yr}$ observing campaign using the Magellan Inamori Kyocera Echelle spectrograph on the Magellan Telescopes at Las Campanas Observatory, the Robert G. Tull Coudé Spectrograph on the Harlan J. Smith Telescope at McDonald Observatory, and the High Resolution Spectrograph on the Hobby-Eberly Telescope at McDonald Observatory. We perform a standard LTE abundance analysis using MARCS model atmospheres, and we apply line-by-line statistical corrections to minimize systematic abundance differences arising when different sets of lines are available for analysis. We identify several abundance correlations with effective temperature. A comparison with previous abundance analyses reveals significant differences in stellar parameters, which we investigate in detail. Our metallicities are, on average, lower by $\approx 0.25$ dex for red giants and $\approx 0.04$ dex for subgiants. Our sample contains 19 stars with $[\mathrm{Fe} / \mathrm{H}] \leqslant-3.5,84$ stars with $[\mathrm{Fe} / \mathrm{H}] \leqslant-3.0$, and 210 stars with $[\mathrm{Fe} / \mathrm{H}] \leqslant-2.5$. Detailed abundances are presented here or elsewhere for $91 \%$ of the 209 stars with $[\mathrm{Fe} / \mathrm{H}] \leqslant-2.5$ as estimated from medium resolution spectroscopy by Beers, Preston, and Shectman. We will discuss the interpretation of these abundances in subsequent papers.
\end{abstract}

Key words: Galaxy: halo - nuclear reactions, nucleosynthesis, abundances - stars: abundances - stars: atmospheres - stars: Population II

Online-only material: color figures, machine-readable and VO tables

\section{INTRODUCTION}

Observations of the high redshift universe reveal carbon, magnesium, and other metals in the clouds of hydrogen that fueled the rising star formation rate density during the first several Gyr after the big bang (e.g., Sargent et al. 1988; Cooke et al. 2011; Matejek \& Simcoe 2012). Surely some of those gas clouds evolve into galaxies like today's Milky Way, whose stellar halos retain a chemical memory of those early epochs of star formation and metal production. If, however, one is interested in studying the early nucleosynthesis of less abundant metals, like holmium (Sneden et al. 1996) or uranium (Cayrel et al. 2001), stars in the Milky Way are the only practical targets. For this and many other reasons, the importance of expanding the inventory of halo stars whose heavy metal abundances are known in great detail has long been recognized.

\subsection{Previous Surveys of Metal-poor Stars}

Noteworthy in regard to the storyline of this study are the surveys of Bond $(1970,1980)$ and Bidelman \& MacConnell (1973). The photographic plates for their objective-prism surveys were taken with the University of Michigan's $0.61 \mathrm{~m}$ Curtis Schmidt

\footnotetext{
* This paper includes data gathered with the $6.5 \mathrm{~m}$ Magellan Telescopes located at Las Campanas Observatory, Chile, and The McDonald Observatory of The University of Texas at Austin. The Hobby-Eberly Telescope is a joint project of the University of Texas at Austin, the Pennsylvania State University, Stanford University, Ludwig-Maximilians-Universität München, and Georg-August-Universität Göttingen.

3 Current address: Department of Astronomy, University of Michigan, 500 Church Street, Ann Arbor, MI 48109, USA.
}

Telescope. This telescope, initially located near Ann Arbor, was relocated in 1966 to Cerro Tololo Inter-American Observatory (CTIO). Most of the observations for Bond (1970) were made in Michigan, while Bidelman \& MacConnell and Bond (1980) made their observations at CTIO. Bond also used Strömgren photometry to assign spectral types and luminosity classes to his candidates, and he measured radial velocities from followup coudé spectroscopy when possible.

Many of the well-known and bright metal-poor stars in the Henry Draper (HD), Bonner Durchmusterung (BD), or Córdoba Durchmusterung (CD) Catalogs were identified during this period by these surveys. Other metal-poor stars were found among the high proper motion stars in the Lowell Proper Motion Survey (Giclas et al. 1971, 1978; these stars are identified with a "G" prefix before their catalog designation) and the New Luyten Two Tenths Catalog (Luyten 1979). Ryan (1989) discusses the methods used to identify metal-poor stars in these surveys.

\subsection{The HK Objective-Prism Survey}

Our own work on the subject began with an objectiveprism survey at the Curtis Schmidt (CS) Telescope at CTIO, initiated by G. Preston and S. Shectman, in 1978-1979. The key advancement was the use of an interference filter to expose only the region around the stellar $\mathrm{Ca} I \mathrm{H}$ and $\mathrm{K}$ absorption lines at a spectral resolution of $\approx 5 \AA(R \equiv \lambda / \Delta \lambda \sim 800)$. This interference filter reduced crowding and sky fog and allowed longer exposure times (90 minutes) than had been practical previously. The "HK" Survey plates reach $B \approx 15$, several magnitudes fainter than the surveys of Bond $(1970,1980)$ and 
Bidelman \& MacConnell (1973). Visual inspection of the plates using a low-power binocular microscope yielded about 1800 metal-poor candidate stars on 72 plates.

Broadband $U B V$ photometry and followup medium resolution $(1 \AA ; R \sim 4000)$ spectroscopy covering the $3700-4500 \AA$ wavelength range were obtained for 450 candidates. Beers et al. (1985) presented metallicity estimates for 134 metal-poor candidates with $[\mathrm{Fe} / \mathrm{H}]<-2.0$. Using a revised metallicity calibration (Beers et al. 1990), Beers et al. (1992, hereafter BPS92) published metallicity estimates, radial velocity (RV) measurements, and distances for 1044 dwarfs and giants with subsolar $[\mathrm{Fe} / \mathrm{H}]$ from 135 unique fields covering 3375 square degrees (8\% of the sky).

The relocation of Case Western Reserve University's Burrell Schmidt (BS) Telescope from Cleveland to Kitt Peak National Observatory in 1979 enabled a similar survey in the Northern Hemisphere. Followup medium resolution spectroscopy for these candidates was not yet available in 1992. Beers (2013) describes the impressive worldwide network of 2-4 m class telescopes involved in the subsequent medium resolution spectroscopic followup of metal-poor candidates from both the northern and southern portions of the HK Survey. Detailed abundances of metal-poor candidates from the northern portion of the HK Survey have been published elsewhere (e.g., Honda et al. 2004a, 2004b; Lai et al. 2004, 2008) and will not be considered in the sample presented here.

An analysis of digital scans of the original HK Survey plates was made by Rhee (2001) and in subsequent unpublished work by J. Rhee, T. Beers, and coworkers (see also Section 3.3.1 of Beers \& Christlieb 2005). The goal of this "HK-II" Survey was to identify metal-poor red giant stars that may have been overlooked in the original visual scans of the plates due to the unavoidable temperature bias against cool stars. These candidates will not be considered in the sample presented here.

\subsection{Subsequent Surveys}

The Hamburg/ESO (HE) Survey (Wisotzki et al. 2000) introduced quantitative methods to identify metal-poor candidates from digitized spectra (Christlieb et al. 2008). These techniques increase the effective yields of genuine metal-poor stars, especially among giants, when color information is included as part of the selection criteria. This survey also reaches several magnitudes deeper than the HK Survey.

Recent surveys have built on these quantitative techniques to identify even greater numbers of candidate metal-poor stars from low resolution spectroscopy, including the Radial Velocity Experiment (RAVE; see Fulbright et al. 2010), the Sloan Digital Sky Survey (SDSS), and the Sloan Extensions for Galactic Understanding and Exploration (SEGUE-1 and SEGUE-2; Yanny et al. 2009; Rockosi 2012). Many metal-poor candidates are also expected to be found among ongoing surveys by the Large sky Area Multi-Object fiber Spectroscopic Telescope (e.g., Deng et al. 2012); and the SkyMapper Telescope (Keller et al. 2012).

\subsection{Detailed Abundance Followup of Metal-poor Candidates}

Bright stars identified by the surveys of Bond (1970, 1980) and Bidelman \& MacConnell (1973) have been analyzed in great chemical detail by numerous investigators, including Luck \& Bond (1981, 1985), Hartmann \& Gehren (1988), Gilroy et al. (1988), Gratton \& Sneden (1988, 1991, 1994), Magain (1989), Peterson et al. (1990), Zhao \& Magain (1990), and Johnson (2002). Ryan et al. (1991), Fulbright (2000, 2002), Stephens
\& Boesgaard (2002), and Ishigaki et al. (2010) studied the compositions of stars selected from the early objective prism and proper motion surveys. Detailed abundance studies of large samples of stars from the HE Survey have been conducted by Carretta et al. (2002), Cohen et al. (2002, 2004, 2008), Barklem et al. (2005), Aoki et al. (2007), Hollek et al. (2011), Norris et al. (2013), and Yong et al. (2013). Candidates from the HK-II Survey have been observed as part of the Chemical Abundances of Stars in the Halo project at the University of Texas (Frebel et al. 2008a; Roederer et al. 2008a). Detailed chemical followup of large numbers of stars from the SDSS and SEGUE have been performed by Aoki et al. (2008, 2013), Caffau et al. (2011), and Bonifacio et al. (2012).

Over the last several decades, high resolution optical spectroscopic followup of candidates from BPS92 has confirmed hundreds of them as genuine metal-poor stars. Chemical abundances of handfuls of stars from Beers et al. (1985) were presented by Molaro \& Bonifacio (1990), Molaro \& Castelli (1990), Norris et al. (1993), and Primas et al. (1994). McWilliam et al. (1995a, 1995b), Norris et al. (1996), and Ryan et al. (1996) were the first to analyze larger samples of stars (34 stars between them) from BPS92. Since then, the number of detailed abundance studies conducted on candidates from BPS92 has grown tremendously, and there are far too many excellent ones to list here individually. There have been several dedicated observing campaigns to obtain high resolution spectroscopy of substantial numbers of stars (typically $\approx 10-30$ ), including analyses by Aoki et al. (2005, 2007), Honda et al. (2004a, 2004b), Lai et al. (2008), the "First Stars" team (Cayrel et al. 2004; Spite et al. 2005; François et al. 2007; Bonifacio et al. 2009), and a reanalysis of the published values from many of these studies by Yong et al. (2013).

The detailed chemical analysis performed by McWilliam et al. (1995a, 1995b) launched our efforts to use these stars as probes of the earliest epoch of metal enrichment in the Galaxy. Our subsequent abundance studies based on high resolution spectroscopy of metal-poor candidates from the HK Survey have examined carbon rich metal-poor stars (Preston \& Sneden 2001; Sneden et al. 2003; Roederer et al. 2014), individual stars of interest (Sneden et al. 1994; Ivans et al. 2005; Preston et al. 2006b; Thompson et al. 2008), stars on the horizontal branch (HB; Preston et al. 2006a), and stars with kinematics indicative of a cold stellar stream (Roederer et al. 2010).

In this paper we present abundance results for 313 stars, including 217 stars from the HK Survey, using high resolution spectroscopy obtained from 2003-2013 at the Magellan Telescopes at Las Campanas Observatory. As of 2013 July, detailed abundances for $91 \%(191 / 209)$ of the stars with estimated $[\mathrm{Fe} / \mathrm{H}] \leqslant-2.5$ in BPS92 are presented here or have been published elsewhere previously. Sixty-one percent (132/217) of the stars from the HK Survey presented in this work are analyzed in such a manner for the first time. Abundances in the other 85 stars have been examined previously by the studies above or others named below. A limited selection of stars from the BD, $\mathrm{CD}, \mathrm{G}, \mathrm{HD}$, and $\mathrm{HE}$ catalogs are also (re)analyzed in the present study.

\section{ABUNDANCE NOTATION}

We adopt standard definitions of elemental abundances and ratios. For element $X$, the logarithmic absolute abundance is defined as the number of atoms of element $\mathrm{X}$ per $10^{12}$ hydrogen atoms, $\log \epsilon(\mathrm{X}) \equiv \log _{10}\left(N_{\mathrm{X}} / N_{\mathrm{H}}\right)+12.0$. For elements $\mathrm{X}$ and $\mathrm{Y}$, 
the logarithmic abundance ratio relative to the solar ratio is defined as $[\mathrm{X} / \mathrm{Y}] \equiv \log _{10}\left(N_{\mathrm{X}} / N_{\mathrm{Y}}\right)-\log _{10}\left(N_{\mathrm{X}} / N_{\mathrm{Y}}\right)_{\odot}$.

Abundances or ratios denoted with the ionization state are defined to be the total elemental abundance as derived from transitions of that particular ionization state after ionization corrections, assuming Saha (1921) equilibrium, have been applied. For example, $\log \epsilon(\mathrm{Fe}$ II $)$ denotes the number density of all iron atoms as derived from Fe II lines.

When reporting relative abundance ratios for a specific element $\mathrm{X}$ (e.g., $[\mathrm{X} / \mathrm{Fe}]$ ), these ratios are constructed by comparing total abundances derived from species in the same ionization state. For example, if $\mathrm{X}$ is a neutral species, the ratio $[\mathrm{X} / \mathrm{Fe}]$ is calculated using the total abundance of element $\mathrm{X}$ derived from the neutral species with the total iron abundance derived from Fe I. Similarly, if $\mathrm{X}$ is an ionized species, the ratio $[\mathrm{X} / \mathrm{Fe}]$ is calculated using the total abundance of element $\mathrm{X}$ derived from the ionized species with the total iron abundance derived from Fe II.

\section{OBSERVATIONS}

\subsection{Target Selection}

Over the course of this program we have observed $88 \%$ $(184 / 209)$ of the stars with estimated $[\mathrm{Fe} / \mathrm{H}] \leqslant-2.5$ in BPS92, excluding three duplicate identifications in Table 5 of BPS92. Abundances derived from previous moderate or high resolution spectroscopy of seven of the remaining 25 stars have been presented elsewhere by Norris et al. (1993), Spite et al. (2000), Preston \& Sneden (2001), Lai et al. (2004), and T. Masseron et al. (unpublished; see Masseron et al. 2010). The 18 remaining unobserved candidates are faint by our standards, $V>14.5$, and most are fainter than $V>15.0$.

We also observed other metal-poor stars to expand the sample to higher metallicity and into the northern hemisphere. These additional targets include higher metallicity candidates from BPS92 and bright stars from the catalogs discussed in Section 1. Some of these stars have been previously analyzed elsewhere. Our final sample includes observations of 217 stars from the HK Survey and 96 stars from other sources.

All stars in our sample are present in the SIMBAD database, and their coordinates may be found there. During the course of the objective-prism survey, portions of some fields were observed multiple times with different plates. This led to multiple identification numbers for a few candidates. In such cases we adopt the number associated with the earliest observation. Table 1 lists the stars in our survey that received multiple identification numbers. Several of these stars were also rediscovered during the course of the HE Survey, and the HE designations are listed in Table 1. The HK Survey itself rediscovered stars with previous catalog names, and these are listed in Table 6 and on page 2033 of BPS92.

\subsection{High Resolution Spectroscopy}

Observations conducted at Las Campanas Observatory were made with the Magellan Inamori Kyocera Echelle (MIKE) spectrograph (Bernstein et al. 2003). This instrument is currently mounted on the $f / 11$ Nasmyth platform at the $6.5 \mathrm{~m}$ Landon Clay Telescope (Magellan II). Early observations for our program were taken while MIKE was mounted on the $6.5 \mathrm{~m}$ Walter Baade Telescope (Magellan I) in 2003. The MIKE spectra were taken with the $0 ! 7 \times 55^{\prime \prime} 0$ slit, yielding a resolving power of $R \sim 41,000$ in the blue and $R \sim 35,000$ in the red as measured from isolated ThAr lines. A dichroic splits the two arms around
Table 1

Repeat Star Identifications

\begin{tabular}{lc}
\hline \hline Primary Name & Additional Designations \\
\hline CS 22169-035 & HE 0409-1212 \\
CS 22172-029 & HE 0328-1047 \\
CS 22185-007 & HE 0315-1528 \\
CS 22189-009 & HE 0239-1340 \\
CS 22873-128 & HE 2002-5843 \\
CS 22886-003 & CS 29512-030 \\
CS 22886-012 & CS 29512-015 \\
CS 22886-013 & CS 29512-013 \\
CS 22888-014 & CS 30493-023 \\
CS 22890-064 & CS 30306-117 \\
CS 22892-052 & HE 2214-1654 \\
CS 22894-023 & CS 22952-011 \\
CS 22937-072 & CS 29501-051 \\
CS 22942-002 & CS 30492-102 \\
CS 22948-066 & CS 30044-2459 $343-064$ \\
CS 22949-037 & HE 2323-0256 \\
CS 22952-015 & HE 2334-0604 \\
CS 22954-015 & HE 0236-0242 \\
CS 22957-027 & HE 2356-0410 \\
CS 22968-014 & HE 0305-5442 \\
CS 29517-042 & CS 31060-052 \\
CS 30339-069 & HE 0027-3613 \\
\hline
\end{tabular}

$4950 \AA$, although the wavelength at the split is bluer in earlier observations. This setup gives approximately 2.4 pixels per resolution element (RE) in the blue and 2.1 pixels $\mathrm{RE}^{-1}$ in the red. Many of the observations taken in 2003 and 2004 were made using the double aperture $0.7 \times 2$ ". 0 slit ("A-B mode"). In this mode, exposures are made with the star in aperture $\mathrm{A}$ and the sky in aperture B simultaneously; the pattern is then reversed for the subsequent exposure. This observing procedure was adopted to optimize sky subtraction for faint targets. This strategy proved unnecessary for our targets, which are not skynoise limited, and all subsequent MIKE observations were made using the single $0.7 \times 55^{\prime \prime} 0$ slit. This setup achieves complete wavelength coverage from 3350-9150 $\AA$, although some of the early exposures only extend to $7250 \AA$ in the red.

For the exposures made in the A-B mode, bias subtraction, flat-fielding, sky and scattered light subtraction, cosmic ray removal, and correction of the slit tilt were accomplished with software written by S.A.S. Aperture extraction, wavelength calibration (derived from ThAr frames taken before or after each stellar integration), co-addition of separate observations, and continuum normalization were performed within the IRAF environment. For the exposures made using the single $0.7 \times 55^{\prime \prime} .0$ slit, data reduction, extraction, and wavelength calibration were performed using the MIKE data reduction pipeline written by D. Kelson (see also Kelson 2003). Observations were often broken into several sub-exposures (not longer than $2400 \mathrm{~s}$ per subexposure). We refer to the addition of these sub-exposures as one observation. Coaddition of repeat observations and continuum normalization were performed within the IRAF environment.

Other observations were made with the Robert G. Tull Coudé Spectrograph (Tull et al. 1995) on the $2.7 \mathrm{~m}$ Harlan J. Smith Telescope at McDonald Observatory. These spectra were taken with the $2^{\prime \prime} .4 \times 8^{\prime \prime} .0$ slit, yielding a resolving power $R \sim$ 33,000 and approximately 4.0 pixels $\mathrm{RE}^{-1}$. This setup delivers complete wavelength coverage from $3700-5700 \AA$, with small gaps between the echelle orders further to the red. For our abundance analysis we only use the spectra blueward of $8000 \AA$. 
Table 2

Log of Observations

\begin{tabular}{|c|c|c|c|c|c|c|c|}
\hline Star & $\begin{array}{l}\text { Telescope/ } \\
\text { Instrument }\end{array}$ & Observer & $\begin{array}{c}\text { Exp. } \\
\text { Time (s) }\end{array}$ & Date & $\begin{array}{l}\text { UT Mid- } \\
\text { exposure }\end{array}$ & $\begin{array}{l}\text { Heliocentric } \\
\text { JD }\end{array}$ & $\begin{array}{l}\text { Heliocentric } \\
V_{\mathrm{r}}\left(\mathrm{km} \mathrm{s}^{-1}\right)\end{array}$ \\
\hline CS 22166-016 & Magellan-Clay/MIKE & GWP & 1600 & 2004 Aug 12 & $10: 19$ & 2453229.934 & -210.0 \\
\hline CS 22169-008 & Magellan-Clay/MIKE & IBT & 4000 & 2004 Sep 23 & $08: 55$ & 2453271.874 & +184.2 \\
\hline CS 22169-035 & Magellan-Baade/MIKE & GWP & 1400 & 2003 Jan 18 & $04: 22$ & 2452657.684 & +15.4 \\
\hline CS 22169-035 & Magellan-Baade/MIKE & GWP & 2400 & 2003 Jan 20 & 02:05 & 2452659.589 & +14.1 \\
\hline CS 22169-035 & Magellan-Clay/MIKE & GWP & 1700 & 2007 Aug 24 & 09:56 & 2454336.914 & +14.1 \\
\hline CS 22171-031 & Magellan-Clay/MIKE & GWP & 2800 & 2008 Sep 10 & 07:59 & 2454719.837 & +38.8 \\
\hline CS 22171-037 & Magellan-Clay/MIKE & IBT & 7200 & 2003 Nov 1 & $05: 53$ & 2452944.750 & -261.4 \\
\hline CS 22171-037 & Magellan-Clay/MIKE & IUR & 7200 & 2012 Aug 26 & $06: 12$ & 2456165.762 & -261.1 \\
\hline
\end{tabular}

(This table is available in its entirety in machine-readable and Virtual Observatory (VO) forms in the online journal. A portion is shown here for guidance regarding its form and content.)

Table 3

Observational Stellar Data

\begin{tabular}{|c|c|c|c|c|c|c|c|c|c|}
\hline Star & $\begin{array}{c}\langle\mathrm{RV}\rangle \\
\left(\mathrm{km} \mathrm{s}^{-1}\right)\end{array}$ & $\begin{array}{l}\text { Binary } \\
\text { Flag }^{\mathrm{a}}\end{array}$ & $\begin{array}{c}\text { Literature } \\
\text { RV Ref. }\end{array}$ & $\begin{array}{c}\text { Total Exp. } \\
\text { Time (s) }\end{array}$ & $\begin{array}{l}\text { No. } \\
\text { Obs. }\end{array}$ & $\begin{array}{c}\mathrm{S} / \mathrm{N} \\
3950 \AA\end{array}$ & $\begin{array}{c}\mathrm{S} / \mathrm{N} \\
4550 \AA\end{array}$ & $\begin{array}{c}\text { S/N } \\
5200 \AA\end{array}$ & $\begin{array}{c}\mathrm{S} / \mathrm{N} \\
6750 \AA\end{array}$ \\
\hline CS 22166-016 & -210.0 & 1 & 1 & 1600 & 1 & 115 & 175 & 95 & 140 \\
\hline CS 22169-008 & +184.2 & 0 & $\ldots$ & 4000 & 1 & 100 & 135 & 70 & 95 \\
\hline CS 22169-035 & +14.5 & 1 & 2 & 5500 & 3 & 75 & 135 & 170 & 285 \\
\hline CS 22171-031 & +38.8 & 0 & $\ldots$ & 2800 & 1 & 60 & 80 & 70 & 115 \\
\hline CS 22171-037 & -261.3 & 1 & $\ldots$ & 14400 & 2 & 95 & 125 & 125 & 180 \\
\hline CS 22172-029 & $\ldots$ & 2 & 3 & 7600 & 2 & 80 & 110 & 105 & 180 \\
\hline
\end{tabular}

Notes.

a Binary flags: (0) unknown binary status; (1) no RV variations detected in multiple epochs; (2) RV variations detected, suspected binary, no systemic velocity listed; (3) spectroscopic binary confirmed by other studies, systemic velocity listed.

References. (1) Giridhar et al. 2001; (2) Bonifacio et al. 2009; (3) Barklem et al. 2005; (4) Honda et al. 2004a; (5) McWilliam et al. 1995b; (6) Lai et al. 2008; (7) Aoki et al. 2002a; (8) Tsangarides et al. 2004; (9) Cohen et al. 2002; (10) Lai et al. 2004; (11) Preston 2009; (12) Preston et al. 2006a; (13) Hollek et al. 2011; (14) Preston \& Sneden 2001; (15) Primas et al. 1994; (16) Norris et al. 2001; (17) Depagne et al. 2002; (18) Preston \& Sneden 2000; (19) Norris et al. 1997; (20) Bonifacio et al. 1998; (21) Roederer et al. 2014; (22) Sivarani et al. 2006; (23) Aoki et al. 2007; (24) Sneden et al. 2003; (25) Aoki et al. 2002b; (26) Sbordone et al. 2010; (27) Aoki et al. 2009; (28) Hill et al. 2002; (29) Carney et al. 2003; (30) Zhang et al. 2009; (31) Latham et al. 2002; (32) Latham et al. 1991; (33) Ito et al. 2013; (34) Carney et al. 2008; (35) Pourbaix et al. 2004; (36) Bonifacio et al. 1999; (37) Norris 1986; (38) Nordström et al. 2004; (39) Gratton \& Sneden 1994; (40) Roederer et al. 2008b; (41) Ryan \& Norris 1991; (42) Aoki et al. 2002c; (43) Van Eck et al. 2003; (44) Lucatello et al. 2005; (45) Cohen et al. 2008; (46) Fulbright 2002.

(This table is available in its entirety in machine-readable and Virtual Observatory (VO) forms in the online journal. A portion is shown here for guidance regarding its form and content.)

Additional observations were made with the High Resolution Spectrograph (HRS; Tull 1998) on the 9.2 m Hobby-Eberly Telescope (Ramsey et al. 1998) at McDonald Observatory. These exposures were taken during the standard queue observing mode (Shetrone et al. 2007). Using the 1".0 slit yields a resolving power $R \sim 30,000$ and approximately 3.1 pixels $\mathrm{RE}^{-1}$. In the bluemost cross-dispersion setting this instrument delivers complete wavelength coverage from 3900-6800 A.

For the data obtained with the Tull Spectrograph and HRS, reduction, extraction, sky and scattered light removal, and wavelength calibration (derived from ThAr exposures taken before or after each stellar exposure) of the spectra were accomplished using the REDUCE software package (Piskunov $\&$ Valenti 2002). These observations were also broken into several sub-exposures with exposure times typically not longer than $1800 \mathrm{~s}$. Coaddition and continuum normalization were performed within the IRAF environment.

We have observed 250 stars with Magellan+MIKE, 52 stars with Smith + Tull, and 19 stars with HET + HRS. After accounting for duplicate observations of the same star and three double-lined spectroscopic binaries whose abundances are not examined in this paper, we are left with a total of 313 stars. These 532 individual observations account for nearly $495 \mathrm{hr}$ of integration time. In Table 2 we present a record of all observations. A full version of Table 2 is available in the online edition of the journal.

Signal-to-noise ratio (S/N) estimates, listed in Table 3, are based on Poisson statistics for the number of photons collected in the continuum at several reference wavelengths once all observations of a given target have been coadded together. Spectra obtained with different instruments were not coadded. These $\mathrm{S} / \mathrm{N}$ estimates are illustrated in Figure 1. We have succeeded in achieving a relatively high $\mathrm{S} / \mathrm{N}$ at $3950 \AA$, between the $\mathrm{Ca}$ II $\mathrm{H}$ and $\mathrm{K}$ lines, for most stars observed with the MIKE and Tull spectrographs ( $\mathrm{S} / \mathrm{N} \gtrsim 50$ and 40 , respectively). The HRS is not optimized for blue response, and similar $\mathrm{S} / \mathrm{N}$ levels were not practical in stars observed with this setup. The $\mathrm{S} / \mathrm{N}$ continues to increase when moving toward the red for stars observed with all three instruments. High $\mathrm{S} / \mathrm{N}$ levels in the blue region of the spectrum are essential for deriving abundances of many species whose most promising transitions are found in this spectral region.

\section{RADIAL VELOCITIES}

To measure the RV of each of our target stars, we cross correlate our spectra against standard template stars using the fxcor task in IRAF. The RV with respect to the ThAr lamp 

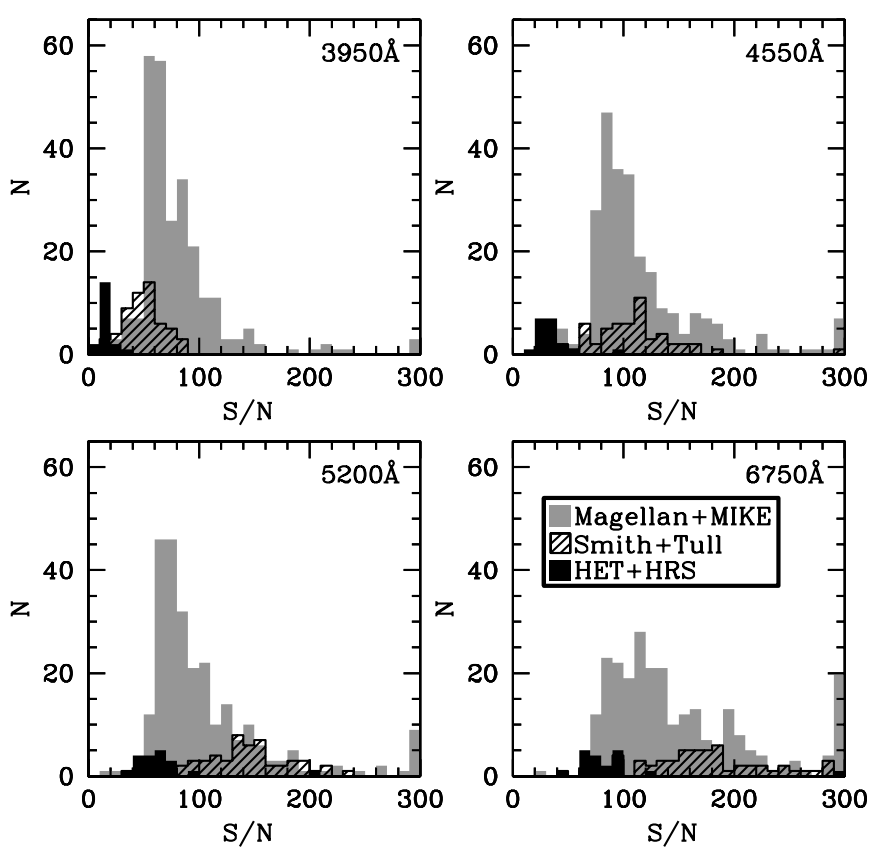

Figure 1. $\mathrm{S} / \mathrm{N}$ estimates at four wavelengths. The gray shaded histogram represents spectra obtained with the MIKE spectrograph, the hatched histogram represents spectra obtained with the Tull spectrograph, and the black shaded histogram represents spectra obtained with the HRS. The last bar to the right in each panel indicates the number of stars with $\mathrm{S} / \mathrm{N}>300 / 1$. is found by cross correlating the echelle order containing the Mg I b lines. For spectra taken with the MIKE and Tull spectrographs, we also cross correlate the echelle order containing the telluric $\mathrm{O}_{2} B$ band near $6900 \AA$ with a template to remove any velocity shifts resulting from thermal and mechanical motions in the spectrographs. We use empirical $\mathrm{O}_{2}$ wavelengths from Griffin \& Griffin (1973) to create this zero-velocity template from one spectrum with high $\mathrm{S} / \mathrm{N}$ ratios obtained with each instrument. The HRS spectra do not contain this band, and we cross-correlate against the telluric $\mathrm{O}_{2} \alpha$ band near $6300 \AA$ using laboratory wavelengths from Babcock \& Herzberg (1948). These measurements are consistent with no shift. Heliocentric corrections are computed using the IRAF rvcorrect task. Heliocentric RV measurements for each observation are listed in Table 2.

Table 3 lists the mean RV derived for each of our targets. We have searched the literature for previous RV measurements to identify stars that are members of binary or multiple star systems. We compare our heliocentric RV measurements to those measured by previous investigators. These references are listed in Table 3. Our measurements for known RV-constant stars are all in good agreement with previous investigations. Typical RV uncertainties for previous studies are $\approx 1 \mathrm{~km} \mathrm{~s}^{-1}$ when the previous measurement was made from high resolution spectra, and RV uncertainties are often several $\mathrm{km} \mathrm{s}^{-1}$ for lower resolution data. Panel A of Figure 2 displays the RV differences that arise from comparison of our mean measurements to those obtained by previous investigations. We only make this comparison when the previous study reported an error less than
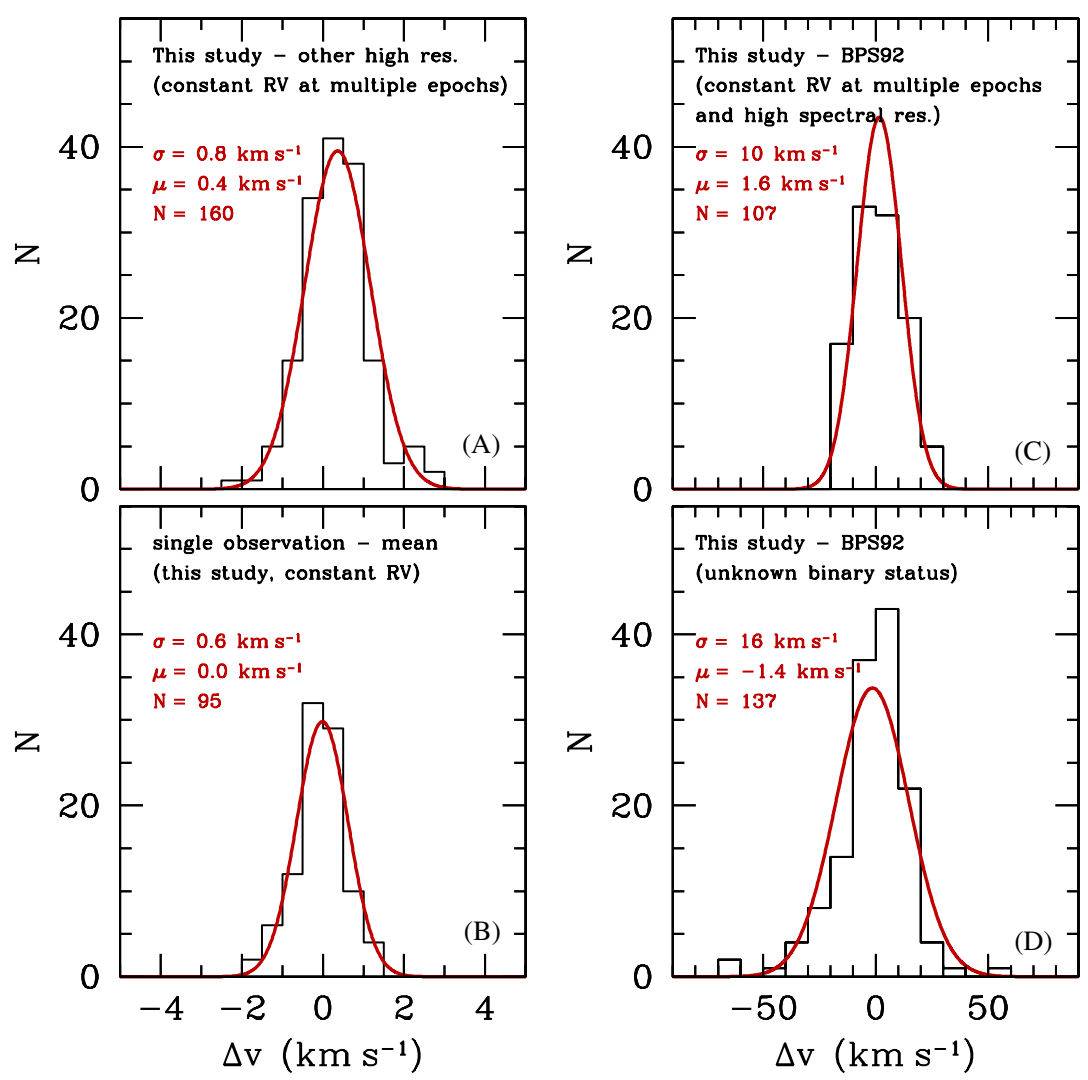

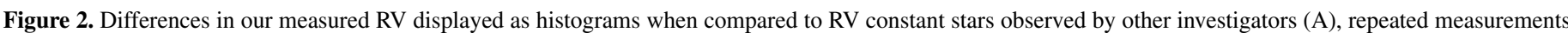

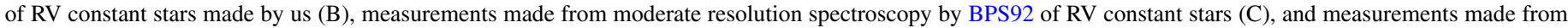

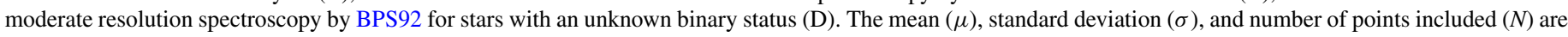
listed in each panel.

(A color version of this figure is available in the online journal.) 
Table 4

Stars with Deviant RV Measurements in BPS92

\begin{tabular}{lccc}
\hline \hline Star & $\begin{array}{c}\text { BPS92 RV } \\
\left(\mathrm{km} \mathrm{s}^{-1}\right)\end{array}$ & $\begin{array}{c}\text { High Res. RV } \\
\left(\mathrm{km} \mathrm{s}^{-1}\right)\end{array}$ & Other Ref. $^{\mathrm{a}}$ \\
\hline CS 22892-052 & -75 & +13.0 & 1,2 \\
CS 22945-058 & -36 & +23.4 & $\ldots$ \\
CS 22949-037 & -79 & -125.4 & 1,3 \\
CS 22949-048 & -95 & -160.8 & 1 \\
CS 22954-004 & +28 & -10.5 & $\ldots$ \\
CS 22956-081 & +210 & +244.6 & $\ldots$ \\
CS 22957-022 & +25 & -31.6 & $\ldots$ \\
CS 22957-024 & -37 & -67.4 & $\ldots$ \\
CS 22957-026 & +14 & -18.9 & $\ldots$ \\
CS 22957-036 & -88 & -154.5 & $\ldots$ \\
\hline
\end{tabular}

\section{Notes.}

a The RV listed in Column 3 is derived from this study. In all cases this value agrees with previous investigations, referenced in Column 4, within $2 \mathrm{~km} \mathrm{~s}^{-1}$. References. (1) McWilliam et al. 1995b; (2) Bonifacio et al. 2009; (3) Depagne et al. 2002; (4) Lai et al. 2008.

$1 \mathrm{~km} \mathrm{~s}^{-1}$; many of these stars also have been observed at many epochs (i.e., $\gg 3$ ), so it is probable that they are indeed RV constant. For the 160 measurements presented in Panel A, $\sigma=$ $0.81 \mathrm{~km} \mathrm{~s}^{-1}$. Panel B compares the differences when a single measurement is compared to the mean of other measurements made by us of the same star for stars that show no RV variations in excess of $2 \mathrm{~km} \mathrm{~s}^{-1}$. This subset has $\sigma=0.64 \mathrm{~km} \mathrm{~s}^{-1}$. For a sample of 95 observations, the total shown in Panel B, and a normal distribution with a dispersion of $0.64 \mathrm{~km} \mathrm{~s}^{-1}$, we would expect less than one observation to deviate by more than $2 \mathrm{~km} \mathrm{~s}^{-1}$. This suggests that $2 \mathrm{~km} \mathrm{~s}^{-1}$ is a reasonable discriminant for identifying RV constant stars at the $\approx 3 \sigma$ level. Based on these tests, we estimate a total uncertainty in each RV measurement of $\approx 0.6-0.8 \mathrm{~km} \mathrm{~s}^{-1}$.

Column 3 of Table 3 indicates the binary status of each star. A flag of " 0 " indicates that the star has only been observed during a single epoch (e.g., one observation, or several observations separated in time by less than one week). For stars lacking $\mathrm{RV}$ variations during this limited time, we assume that we cannot distinguish between a single star or a multiple star system with a relatively long orbital period, so we classify the binary nature of these stars as unknown. A flag of " 1 " indicates that the star has been observed in multiple epochs, either by us or in combination with previous investigators, and does not exhibit any RV variations beyond the mutual uncertainties of the measurements. A flag of " 2 " indicates that the star exhibits $\mathrm{RV}$ variations, either in our measurements or in combination with previous investigators. These stars are likely in binary or multiple star systems, but we lack sufficient information to determine an orbital solution or systemic RV. A flag of " 3 " indicates that the star has previously been identified as a member of a binary or multiple star system. For these stars, we list the systemic velocity as determined by Preston \& Sneden (2000, 2001), Latham et al. (2002), Carney et al. (2003), Sneden et al. (2003), Pourbaix et al. (2004), or Roederer et al. (2014).

Many of our targets from the HK Survey have not been observed previously at high spectral resolution, but BPS92 made RV estimates from moderate resolution $(\sim 1 \AA)$ spectroscopy. Figure 2 also displays the differences in RV measured from the moderate resolution and high resolution spectroscopy. Panel $\mathrm{C}$ shows the differences for stars that have been observed at high spectral resolution at multiple epochs (either by us or in
Table 5

Sample Table of EW Measurements

\begin{tabular}{lcccrc}
\hline \hline Star & $\begin{array}{c}\text { Wavelength } \\
(\AA)\end{array}$ & Species & $\begin{array}{c}\text { E.P. } \\
(\mathrm{eV})\end{array}$ & $\log g f$ & $\begin{array}{c}\text { EW }^{\mathrm{a}} \\
(\mathrm{m} \AA)\end{array}$ \\
\hline CS 22166-016 & 6707.80 & $\mathrm{Li} \mathrm{I}_{\mathrm{I}}$ & 0.00 & 0.17 & Limit \\
CS 22166-016 & 6300.30 & {$\left[\mathrm{O}_{\mathrm{I}}\right]$} & 0.00 & -9.78 & $\ldots$ \\
CS 22166-016 & 7771.94 & $\mathrm{O}_{\mathrm{I}}$ & 9.14 & 0.37 & $\ldots$ \\
CS 22166-016 & 7774.17 & $\mathrm{O}_{\mathrm{I}}$ & 9.14 & 0.22 & $\ldots$ \\
CS 22166-016 & 7775.39 & $\mathrm{O}_{\mathrm{I}}$ & 9.14 & 0.00 & $\ldots$ \\
CS 22166-016 & 4057.51 & $\mathrm{Mg}_{\mathrm{I}}$ & 4.35 & -0.89 & 24.5 \\
CS 22166-016 & 4167.27 & $\mathrm{Mg}_{\mathrm{I}}$ & 4.35 & -0.71 & 39.4 \\
CS 22166-016 & 4702.99 & $\mathrm{Mg}_{\mathrm{I}}$ & 4.33 & -0.38 & $\ldots$ \\
CS 22166-016 & 5172.68 & $\mathrm{Mg}_{\mathrm{I}}$ & 2.71 & -0.45 & 180.1 \\
CS 22166-016 & 5183.60 & $\mathrm{Mg}_{\mathrm{I}}$ & 2.72 & -0.24 & 195.4 \\
CS 22166-016 & 5528.40 & $\mathrm{Mg}_{\mathrm{I}}$ & 4.34 & -0.50 & 58.8 \\
CS 22166-016 & 5711.09 & $\mathrm{Mg}_{\mathrm{I}}$ & 4.34 & -1.72 & $\ldots$ \\
CS 22166-016 & 3943.99 & $\mathrm{Al} \mathrm{I}_{\mathrm{I}}$ & 0.00 & -0.64 & $\ldots$ \\
CS 22166-016 & 3961.52 & $\mathrm{Al} \mathrm{I}_{\mathrm{I}}$ & 0.01 & -0.34 & Synth \\
\hline
\end{tabular}

Notes.

a "Synth" indicates an abundance was derived from spectrum synthesis; "limit" indicates that an upper limit on the abundance was derived from the line.

(This table is available in its entirety in machine-readable and Virtual Observatory (VO) forms in the online journal. A portion is shown here for guidance regarding its form and content.)

combination with other investigators) and do not exhibit any RV variations as measured from the high resolution spectroscopy. The standard deviation, $10 \mathrm{~km} \mathrm{~s}^{-1}$, is identical to the uncertainty estimate reported by BPS92, and the mean offset is only $1.6 \mathrm{~km} \mathrm{~s}^{-1}$. This indicates that their estimates are generally reliable. Panel D shows the differences for stars that have only been observed at high spectral resolution at a single epoch. The larger $\sigma$ of this sample, $18 \mathrm{~km} \mathrm{~s}^{-1}$, leads us to speculate that at least some of these stars may be in binary or multiple star systems; however, caution is warranted regarding this conclusion. Several of the RV estimates reported in BPS92 deviate significantly from the RV measurements derived from high resolution spectroscopy. Stars whose RV estimate in BPS92 differs by more than $30 \mathrm{~km} \mathrm{~s}^{-1}$ from our high resolution RV measurements are listed in Table 4 and are not shown in Figure 2. Barring any misidentifications in this survey, we attribute these discrepancies to irregularities of unknown origin that occurred during the course of the BPS92 survey. In light of this, we refrain from assigning a binary status classification to any star with only a single high resolution RV measurement, even if the star has an RV estimate from BPS92.

Finally, our observations reveal two new double-lined spectroscopic binary stars, CS 22884-033 and HE 2047-5612. We also reaffirm earlier work by Masseron et al. (2012) showing that the spectrum of CS 22949-008 exhibits two sets of lines. We present the observational data for these three stars in Table 2, but we omit them from the subsequent analysis.

\section{EQUIVALENT WIDTHS}

We measure equivalent widths (EWs) from our spectra using a semi-automatic routine that fits Voigt absorption line profiles to continuum-normalized spectra. This routine presents a plot of every fit to the user for final approval or modification. The local continuum is identified automatically by an iterative clipping procedure using a region of $3.5 \AA$ on either side of the line of interest, but the user can identify a different continuum level for each line when necessary. Typically $7-11$ points surrounding the 

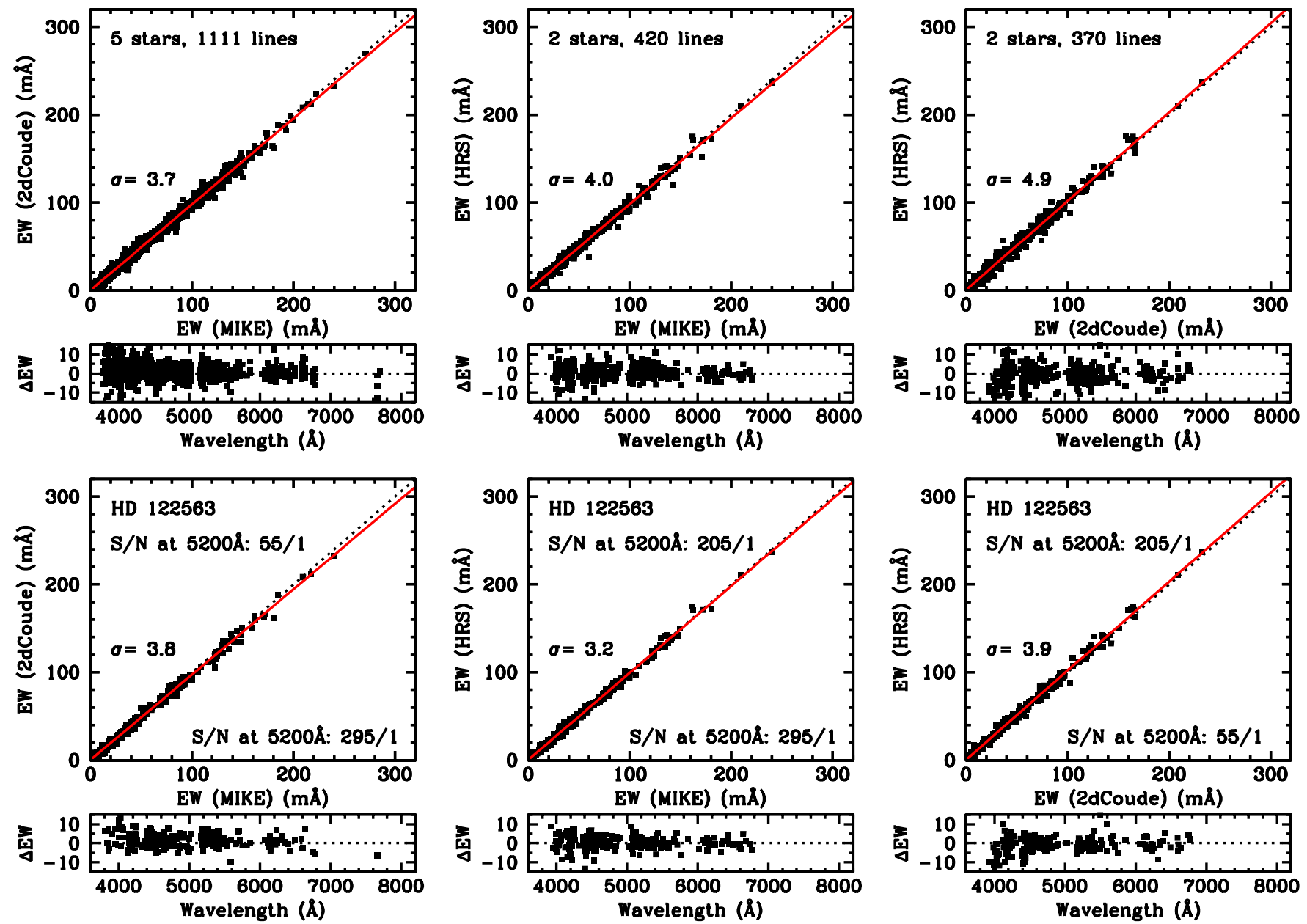

Figure 3. Comparison of EWs measured from spectra of the same stars obtained with different spectrographs. The 1:1 correlation is indicated as a dotted line, and the least-squares fit is indicated by the solid red line. The standard deviation $(\sigma)$ is computed relative to the 1:1 correlation, not the least-squares fit. The top row of panels examines the combined measurement for stars observed with both the MIKE and Tull ("2dCoude") spectrographs (HD 106373, HD 108317, HD 122563, HD 132475, and HE 1320-1339), MIKE and HRS (G015-010 and HD 122563), and the Tull and HRS (G025-024 and HD 122563). The bottom row of panels examines the measurements for one star observed with all three instruments, HD 122563.

(A color version of this figure is available in the online journal.)

line center are used for the fit, covering 3-4 times the Gaussian FWHM depth of the line for weak lines. For stronger lines with clearly visible wings and no obvious blending features, the number of fitting points is increased. We choose to fit Voigt profiles to the absorption lines to simultaneously account for the Gaussian core and the dispersion wings present in some stronger lines; for weak lines, the Voigt profile effectively resembles a Gaussian profile.

The complete list of 47,744 EW measurements for all stars analyzed is given in Table 5. Other lines fit by spectrum synthesis (9268 lines) or used to derive upper limits (12,279 lines) are indicated using "synth" or "limit," respectively. The complete version of Table 5 is available in the online edition of the journal, and only a sample is shown in the printed edition to demonstrate its form and content.

\subsection{Comparison of Equivalent Widths Measured from Spectra Obtained with Different Spectrographs}

We have deliberately observed a small number of bright stars with more than one instrument to check the reliability of the reduction processes at handling cosmic rays, scattered light, flatfielding, etc. We have five stars in common to Magellan + MIKE and Smith + Tull, two stars in common to Magellan + MIKE and HET + HRS, and two stars in common to HET + HRS and Smith + Tull; one star, HD 122563, was observed with all three setups. In Figure 3 we compare the EWs measured from each of these spectra. The standard deviation of each set of comparisons is driven strongly by the $\mathrm{S} / \mathrm{N}$ of the individual spectra. The largest deviations regularly arise from strong lines that suffer from blending and lower $\mathrm{S} / \mathrm{N}$ in the blue. We find no evidence of differences in the spectra beyond the EW measurement uncertainties, typically 3-4 mA. It is reassuring that the spectra obtained with the different instruments are effectively interchangeable in the wavelength regions where they overlap with adequate $\mathrm{S} / \mathrm{N}$.

\subsection{Comparison of Equivalent Widths with Those Measured by Other Investigators}

Our sample has many stars in common with earlier studies by other investigators. Figure 4 compares EWs for 12 of the stars in common with the First Stars sample (Cayrel et al. 2004). For 1808 lines in common with $3700 \AA<\lambda<6500 \AA$ and $0 \mathrm{~m} \AA<\mathrm{EW}<220 \mathrm{~m} \AA$, we find a standard deviation $(\sigma)$ of $4.7 \mathrm{~m} \AA$ and a very slight trend for the strongest lines (EW $\gtrsim$ $150 \mathrm{m \AA}$ ), where our EW measurements are larger by $\approx 4 \%-5 \%$ on average. The weakest lines $(\mathrm{EW}<100 \mathrm{m \AA})$ show very 
Table 6

Comparison of Equivalent Width Measurements

\begin{tabular}{|c|c|c|c|c|c|c|c|}
\hline Study & Line Subset & $N_{\text {stars }}$ & $N_{\text {lines }}$ & Slope & Zeropoint & Mean Offset & $\sigma(\mathrm{m} \AA)$ \\
\hline \multirow[t]{2}{*}{ Carretta et al. (2002) } & All lines & 2 & 155 & 0.88 & 5.2 & +2.3 & 7.0 \\
\hline & $\mathrm{EW}<100 \mathrm{m \AA}$ & 2 & 123 & 0.88 & 5.2 & +0.3 & 5.3 \\
\hline \multirow[t]{2}{*}{ Cayrel et al. (2004) } & All lines & 12 & 1808 & 0.95 & 1.5 & +1.8 & 4.7 \\
\hline & $\mathrm{EW}<100 \mathrm{m \AA}$ & 12 & 1415 & 0.96 & 0.8 & +0.7 & 3.6 \\
\hline \multicolumn{8}{|l|}{ Honda et al. (2004a) } \\
\hline \multirow[t]{2}{*}{ Bright targets } & All lines & 2 & 276 & 0.95 & 1.2 & +1.7 & 3.7 \\
\hline & $\mathrm{EW}<100 \mathrm{m \AA}$ & 2 & 231 & 0.95 & 1.2 & +1.0 & 3.0 \\
\hline \multirow[t]{2}{*}{ Faint targets } & All lines & 2 & 176 & 0.85 & 7.0 & +1.9 & 9.6 \\
\hline & $\mathrm{EW}<100 \mathrm{m \AA}$ & 2 & 137 & 0.86 & 6.6 & -0.9 & 7.2 \\
\hline \multirow[t]{2}{*}{ Ivans et al. (2003) } & All lines & 2 & 262 & 0.96 & 1.5 & +0.3 & 4.2 \\
\hline & $\mathrm{EW}<100 \mathrm{m \AA}$ & 2 & 241 & 0.94 & 1.8 & +0.1 & 3.8 \\
\hline \multirow[t]{2}{*}{ Johnson (2002) } & All lines & 3 & 763 & 0.93 & 1.3 & +1.9 & 5.0 \\
\hline & $\mathrm{EW}<100 \mathrm{m \AA}$ & 3 & 693 & 0.95 & 0.6 & +1.3 & 3.4 \\
\hline \multirow[t]{2}{*}{ Lai et al. (2008) } & All lines & 3 & 397 & 0.97 & 0.5 & +1.3 & 4.2 \\
\hline & $\mathrm{EW}<100 \mathrm{m \AA}$ & 3 & 355 & 0.97 & 0.4 & +1.0 & 3.6 \\
\hline \multirow[t]{2}{*}{ McWilliam et al. (1995b) } & All lines & 8 & 1350 & 0.93 & 5.1 & -0.3 & 11.3 \\
\hline & $\mathrm{EW}<100 \mathrm{m \AA}$ & 8 & 1033 & 0.94 & 4.7 & -1.6 & 9.6 \\
\hline
\end{tabular}
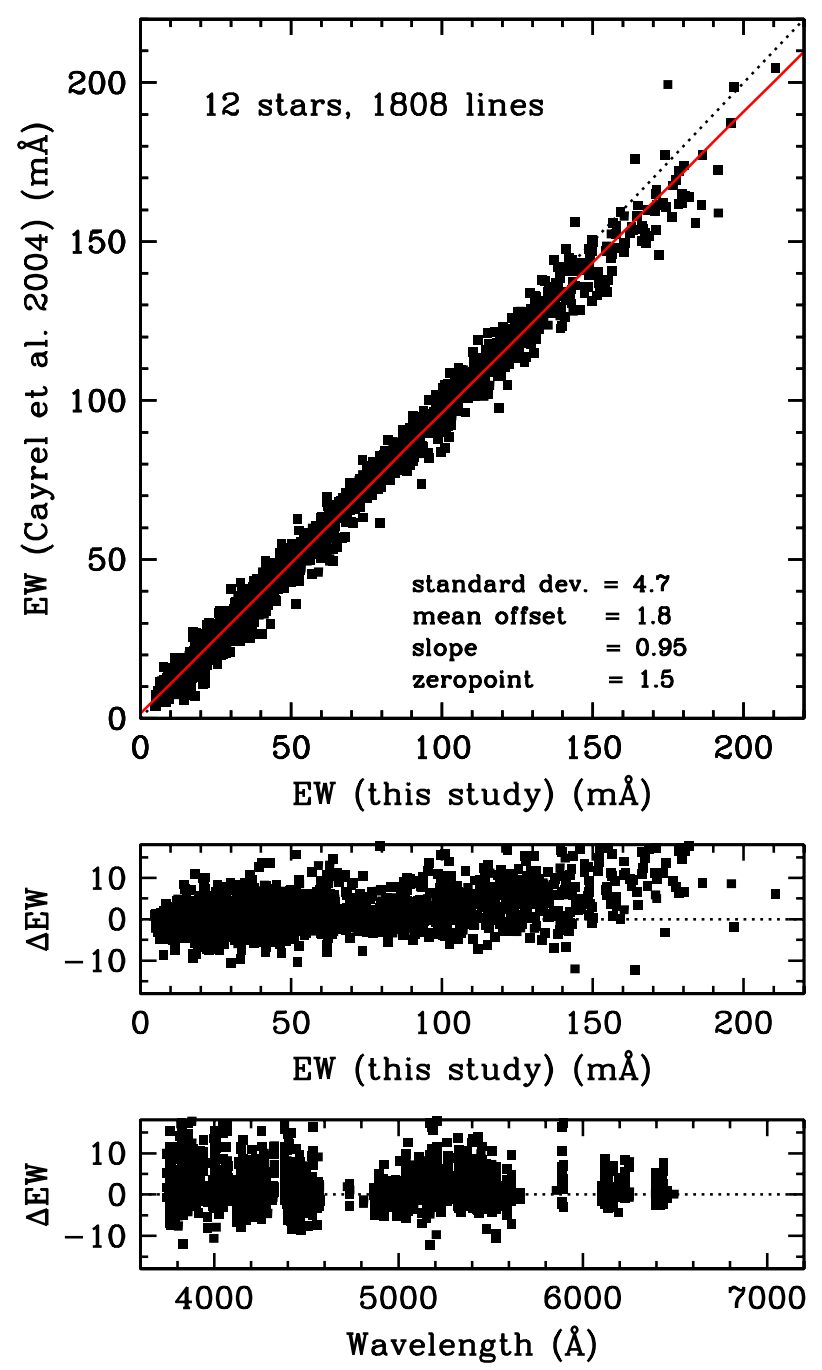

Figure 4. Comparison of EW measurements with those of Cayrel et al. (2004). The 1:1 correlation is indicated as a dotted line, and the least-squares fit is indicated by the solid red line in the top panel. The standard deviation is computed relative to the 1:1 correlation, not the least-squares fit. The differences are in the sense of this study minus Cayrel et al.

(A color version of this figure is available in the online journal.) good agreement and have a smaller $\sigma, 3.6 \mathrm{~m} \AA$. This standard deviation is the same as found in comparisons of EWs measured by us using spectra from different instruments. No significant variations from these values are found when EWs from these 12 stars are considered on a star-by-star basis. Similar results are obtained when we compare our EWs with those of other investigators. Table 6 lists the standard deviation found when our EWs are compared with those of stars in common for six other studies. Table 6 also lists the slope and zeropoint of the linear regression (where 1.0 and 0.0 represent the slope and zeropoint for perfect agreement) and the mean offset. The slopes range from 0.88 to 0.97 with positive zeropoints; our EWs are systematically larger for the strongest lines. The mean offsets are negligible. The large standard deviation with respect to McWilliam et al. (1995b) is a result of the relatively low $\mathrm{S} / \mathrm{N}$ of the spectra in that study (typical $\mathrm{S} / \mathrm{N}$ of $30-45$ pixel $^{-1}$ ). For most other studies, $\sigma$ for all lines is $\approx 4.0-5.0 \mathrm{~m} \AA$; when only weak lines are considered (EW $<100 \mathrm{~mA}), \sigma$ drops to $\approx 3.5-4.0 \mathrm{~m} \AA$.

To further investigate the nature of the discrepancy for the strong lines, we examine in detail 20 of the strongest lines commonly used for abundance analyses in the bright, metal-poor giant HD 128279 from a high S/N MIKE spectrum. Figure 5 compares our EW measurements for these lines with measurements made by fitting Voigt and Gaussian profiles in IRAF, by fitting a Voigt profile in SPECTRE (Fitzpatrick \& Sneden 1987), and by direct integration in SPECTRE via Simpson's rule. We find good agreement between the EW measurements from the Voigt profiles and by direct integration, even for the strongest lines with EW > $250 \mathrm{~m} \AA$. Gaussian profiles clearly underestimate absorption in the wings of the strongest lines. Other investigations do account for extra absorption in the line wings, even if the formal Voigt profile is not explicitly invoked. We conclude that the small systematic difference for the strongest lines results from the different methods used to account for this extra absorption.

\section{PHOTOMETRY}

Optical and infrared broadband photometry is available for most stars in our sample, but these data are only used to calculate initial guesses for the stellar temperatures. Reddening values are taken from the Schlegel et al. (1998) maps, and altered 

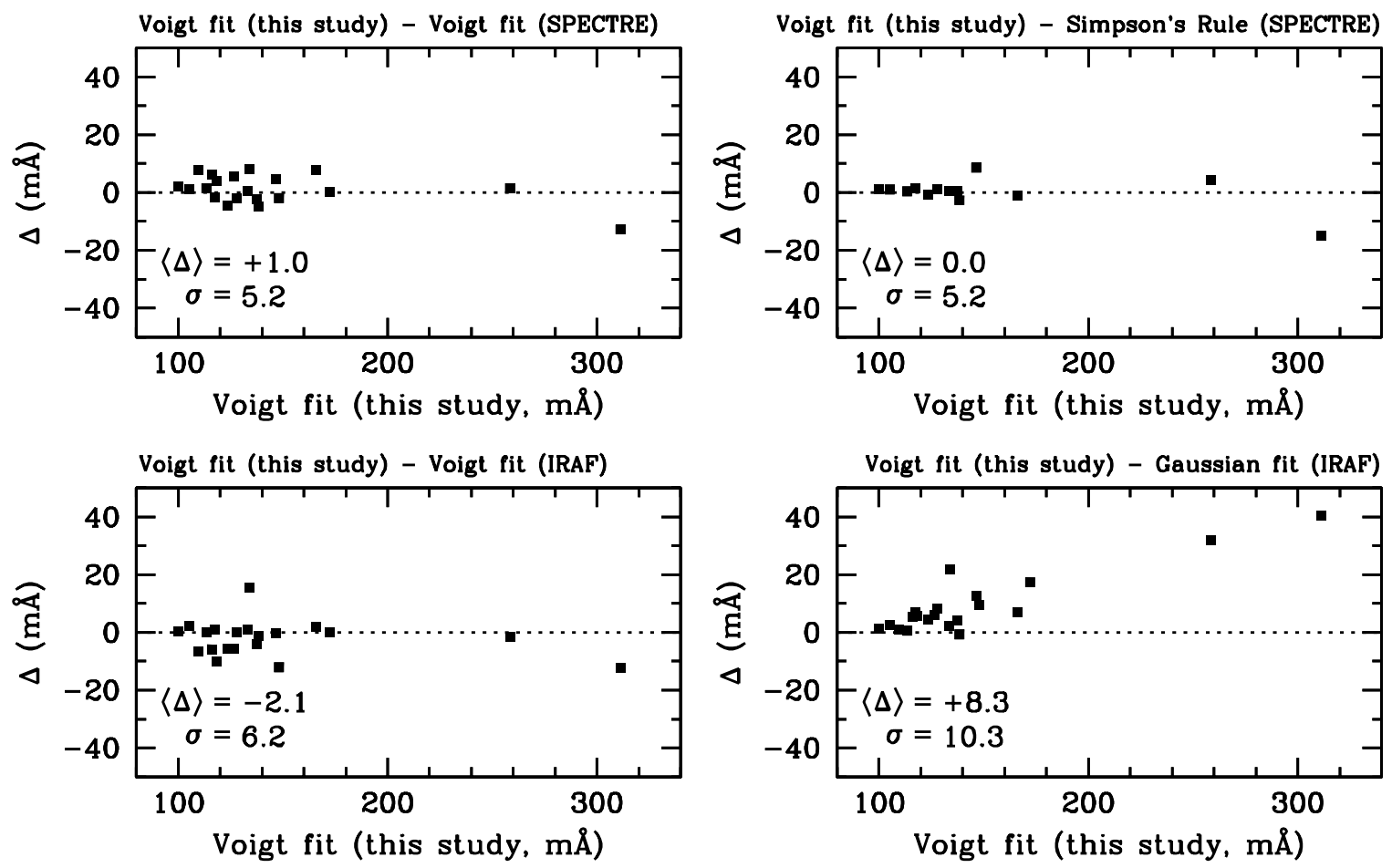

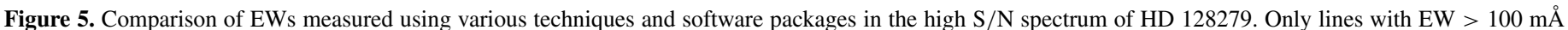

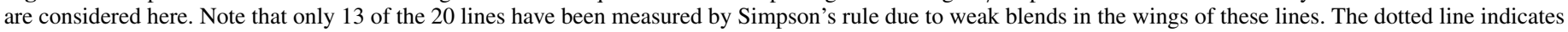
a difference of zero. The mean offset and standard deviation of the residuals are shown.

in the case of high reddening according to the prescription of Bonifacio et al. (2000). We adopt the extinction coefficients of Cardelli et al. (1989). We employ the Ramírez \& Meléndez (2005) color transformations to place the Two Micron All Sky Survey (2MASS; Skrutskie et al. 2006) infrared photometry on the Telescopio Carlos Sánchez scale of Alonso et al. (1999b). The optical photometry originates from many different sources, including Harris \& Upgren (1964), Sandage (1969), Nicolet (1978), Carney (1979, 1983), Carney \& Aaronson (1979), Bond (1980), Norris et al. (1985, 1999), Carney \& Latham (1986), Sandage \& Kowal (1986), Lazauskaite \& Tautvaisiene (1990), Preston et al. (1991, 1994, 2006a), BPS92, Carney et al. (1994), Beers \& Sommer-Larsen (1995), Beers et al. (2000, 2007), Schuster et al. (2004), Rossi et al. (2005), and Christlieb et al. (2008). The $V$ magnitudes, adopted reddenings, and dereddened $B-V$ and $V-K$ colors are listed in Table 7 .

\section{ATOMIC DATA}

We start with a list of 474 lines to be considered for each star, but all lines are not observed, detected, and unblended in each star. This list is found in Table 8. The full version of Table 8 is available only in the online edition of the journal. This table includes the line wavelength, species identification, excitation potential (E.P.) of the lower level of the transition, $\log g f$ value, and references to the source for the $\log g f$ value. We perform spectral synthesis for lines broadened by hyperfine splitting (hfs) or in cases where a significant isotope shift (IS) may be present (see Section 9). The references for these data are also included in Table 8. When available, we use damping constants from Barklem et al. (2000) and Barklem \& AspelundJohansson (2005). In all other cases we resort to the standard Unsöld (1955) approximation.

\section{MODEL ATMOSPHERES}

Numerous challenges in modeling stellar atmospheres persist, and they limit the accuracy of the derived abundances. These challenges include departures from local thermodynamic equilibrium (LTE) in the stellar atmosphere, time-dependent three-dimensional (3D) versus static one-dimensional (1D) representations of the atmosphere, inadequate treatment of convection, inclusion and proper calculation of all relevant sources of continuous opacity, and inaccurate or incomplete atomic and molecular data. Significant progress has been made to improve the situation in recent years, but it is still impractical to analyze large data sets except by means of the general procedures in common use for decades: namely, EW analysis or spectral synthesis of lines assuming 1D, plane-parallel, static model atmospheres in LTE throughout the line-forming layers. We make use of a recent set of MARCS models (Gustafsson et al. 2008), and we demonstrate in Section 8.2 that very similar results are obtained from the ATLAS9 grid (Castelli \& Kurucz 2003).

\subsection{Model Atmosphere Parameters}

The inhomogeneous nature of the optical photometry (Section 6) leads us to adopt model atmosphere parameters that are primarily derived from our spectra. Effective temperatures $\left(T_{\text {eff }}\right)$ are derived by requiring that abundances derived from Fe I lines show no trend with the E.P. of the lower level of the transition. In many stars the $\mathrm{Fe}$ I lines span a range of 0.0 to $4.5 \mathrm{eV}$, a far broader range than is available for any other species.

Microturbulence velocities $\left(v_{\mathrm{t}}\right)$ are derived by requiring that abundances derived from Fe I lines show no trend with line strength, expressed as the unitless quantity $\log (\mathrm{EW} / \lambda)$. Lines with $\log (\mathrm{EW} / \lambda) \gtrsim-5.0$ (e.g., $\mathrm{EW} \gtrsim 40 \mathrm{~m} \AA$ at $\lambda=4000 \AA$ ) are sensitive to $v_{\mathrm{t}}$, and the sensitivity to $v_{\mathrm{t}}$ diminishes on the damping portion of the curve of growth, with $\log (\mathrm{EW} / \lambda) \gtrsim-4.4$ 
Table 7

Magnitudes, Reddenings, Colors, Atmospheric Parameters, and Random Uncertainties

\begin{tabular}{|c|c|c|c|c|c|c|c|c|c|c|c|c|c|}
\hline Class $^{\mathrm{a}}$ & Star & $V$ & $E(B-V)$ & $(B-V)_{0}$ & $(V-K)_{0}$ & $\begin{array}{l}T_{\text {eff }} \\
(\mathrm{K})\end{array}$ & $\log g$ & $\begin{array}{c}v_{\mathrm{t}} \\
\left(\mathrm{km} \mathrm{s}^{-1}\right)\end{array}$ & {$[\mathrm{M} / \mathrm{H}]$} & $\begin{array}{l}\sigma_{T_{\text {eff }}} \\
(\mathrm{K})\end{array}$ & $\sigma_{\log g}$ & $\begin{array}{c}\sigma_{v_{\mathrm{t}}} \\
\left(\mathrm{km} \mathrm{s}^{-1}\right)\end{array}$ & $\sigma_{[\mathrm{M} / \mathrm{H}]^{\mathrm{b}}}$ \\
\hline RG & CS 22166-016 & 12.75 & 0.025 & 0.63 & 1.92 & 4900 & 1.75 & 1.50 & -3.09 & 37 & 0.14 & 0.06 & 0.06 \\
\hline SG & CS 22169-008 & 14.99 & 0.057 & 0.37 & 1.27 & 5810 & 3.60 & 1.30 & -2.59 & 54 & 0.16 & 0.06 & 0.06 \\
\hline RG & CS 22169-035 & 12.88 & 0.043 & 0.89 & 2.41 & 4480 & 0.50 & 1.70 & -3.12 & 34 & 0.14 & 0.06 & 0.06 \\
\hline SG & CS 22171-031 & 13.93 & 0.023 & 0.37 & 1.22 & 6010 & 3.70 & 1.35 & -2.42 & 53 & 0.21 & 0.06 & 0.06 \\
\hline SG & CS 22171-037 & 14.93 & 0.020 & 0.33 & 1.11 & 6110 & 3.65 & 1.60 & -3.35 & 40 & 0.15 & 0.06 & 0.09 \\
\hline RG & CS 22172-029 & 14.36 & 0.056 & $\ldots$ & 1.81 & 4960 & 1.90 & 1.30 & -2.70 & 37 & 0.16 & 0.06 & 0.06 \\
\hline SG & CS 22174-020 & 15.06 & 0.040 & 0.36 & 1.18 & 5840 & 3.55 & 1.40 & -3.02 & 58 & 0.16 & 0.06 & 0.06 \\
\hline SG & CS 22177-009 & 14.27 & 0.044 & 0.36 & 1.20 & 5940 & 3.55 & 1.25 & -3.37 & 38 & 0.16 & 0.06 & 0.08 \\
\hline SG & CS 22177-010 & 14.31 & 0.050 & 0.35 & 1.25 & 6050 & 3.70 & 1.50 & -2.88 & 46 & 0.18 & 0.06 & 0.07 \\
\hline SG & CS 22180-014 & 13.58 & 0.021 & 0.41 & 1.38 & 5780 & 3.55 & 1.50 & -2.90 & 43 & 0.16 & 0.06 & 0.07 \\
\hline SG & CS 22182-033 & 14.67 & 0.033 & 0.41 & 1.41 & 5810 & 3.60 & 1.30 & -2.47 & 47 & 0.16 & 0.06 & 0.06 \\
\hline SG & CS 22182-047 & 13.24 & 0.047 & 0.43 & 1.43 & 5640 & 3.60 & 1.05 & -1.99 & 48 & 0.17 & 0.06 & 0.06 \\
\hline RG & CS 22183-031 & 13.62 & 0.039 & 0.63 & 1.93 & 4850 & 1.60 & 1.55 & -3.50 & 36 & 0.14 & 0.06 & 0.06 \\
\hline RG & CS 22185-007 & 13.34 & 0.047 & 0.64 & 2.08 & 4730 & 1.30 & 1.55 & -3.02 & 38 & 0.15 & 0.06 & 0.06 \\
\hline SG & CS 22186-002 & 13.77 & 0.014 & 0.40 & 1.33 & 5500 & 3.35 & 1.15 & -2.50 & 38 & 0.18 & 0.06 & 0.06 \\
\hline SG & CS 22186-017 & 13.53 & 0.021 & 0.41 & 1.35 & 5770 & 3.55 & 1.30 & -2.90 & 50 & 0.15 & 0.06 & 0.07 \\
\hline RG & CS 22186-023 & 12.84 & 0.022 & 0.66 & 1.96 & 4820 & 1.55 & 1.60 & -2.89 & 34 & 0.12 & 0.06 & 0.06 \\
\hline RG & CS 22189-009 & 14.06 & 0.025 & 0.73 & 2.19 & 4540 & 0.60 & 1.65 & -3.85 & 34 & 0.14 & 0.06 & 0.06 \\
\hline $\mathrm{HB}$ & CS 22191-029 & 14.05 & 0.019 & 0.39 & 1.39 & 5810 & 1.55 & 2.80 & -2.94 & 44 & 0.36 & 0.06 & 0.06 \\
\hline SG & CS 22871-104 & 14.91 & 0.116 & 0.31 & 1.04 & 6370 & 4.00 & 1.40 & -2.06 & 48 & 0.48 & 0.06 & 0.06 \\
\hline SG & CS 22872-102 & 13.65 & 0.231 & 0.36 & 1.40 & 6020 & 3.65 & 1.40 & -2.82 & 49 & 0.16 & 0.06 & 0.07 \\
\hline SG & CS 22873-072 & 14.64 & 0.066 & 0.34 & 1.14 & 6030 & 3.70 & 1.40 & -2.86 & 42 & 0.15 & 0.06 & 0.06 \\
\hline RG & CS 22873-128 & 13.05 & 0.036 & 0.66 & 2.08 & 4710 & 1.20 & 1.65 & -3.24 & 34 & 0.14 & 0.06 & 0.06 \\
\hline SG & CS 22874-123 & 14.70 & 0.105 & 0.34 & 1.24 & 6240 & 3.80 & 1.65 & -2.61 & 40 & 0.20 & 0.06 & 0.06 \\
\hline HB & CS 22875-029 & 13.69 & 0.013 & 0.39 & 1.39 & 5990 & 1.85 & 2.80 & -2.69 & 44 & 0.27 & 0.06 & 0.06 \\
\hline SG & CS 22876-040 & 15.09 & 0.015 & 0.39 & 1.20 & 6090 & 3.80 & 1.45 & -2.21 & 50 & 0.18 & 0.06 & 0.06 \\
\hline RG & CS 22877-001 & 12.16 & 0.057 & 0.71 & 2.01 & 4790 & 1.45 & 1.55 & -3.24 & 34 & 0.14 & 0.06 & 0.06 \\
\hline RG & CS 22877-011 & 13.86 & 0.038 & 0.56 & 1.88 & 4950 & 1.90 & 1.45 & -3.12 & 37 & 0.14 & 0.06 & 0.06 \\
\hline SG & CS 22877-015 & 13.23 & 0.040 & 0.36 & 1.23 & 6150 & 3.85 & 1.30 & -2.00 & 43 & 0.22 & 0.06 & 0.06 \\
\hline SG & CS 22877-051 & 14.26 & 0.051 & 0.29 & 1.19 & 6050 & 3.70 & 1.50 & -2.77 & 46 & 0.17 & 0.06 & 0.07 \\
\hline SG & CS 22878-002 & 14.36 & 0.076 & 0.37 & 1.24 & 5850 & 3.55 & 1.30 & -3.10 & 41 & 0.18 & 0.06 & 0.07 \\
\hline SG & CS 22878-027 & 14.41 & 0.067 & 0.37 & 1.26 & 5820 & 3.55 & 1.20 & -2.97 & 51 & 0.15 & 0.06 & 0.07 \\
\hline RG & CS 22878-101 & 13.73 & 0.100 & 0.75 & 2.21 & 4650 & 1.05 & 1.90 & -3.30 & 35 & 0.14 & 0.06 & 0.06 \\
\hline SG & CS 22879-012 & 14.72 & 0.035 & 0.35 & 1.22 & 6020 & 3.65 & 1.25 & -2.81 & 49 & 0.14 & 0.06 & 0.07 \\
\hline SG & CS 22879-029 & 14.43 & 0.042 & 0.38 & 1.19 & 5920 & 3.70 & 1.30 & -2.35 & 41 & 0.17 & 0.06 & 0.06 \\
\hline SG & CS 22879-051 & 13.89 & 0.039 & 0.35 & 1.16 & 6190 & 3.75 & 1.50 & -2.85 & 51 & 0.22 & 0.06 & 0.07 \\
\hline SG & CS 22879-094 & 15.20 & 0.046 & 0.37 & 1.33 & 5820 & 3.60 & 1.35 & -2.81 & 51 & 0.14 & 0.06 & 0.06 \\
\hline $\mathrm{HB}$ & CS 22879-097 & 14.22 & 0.048 & 0.41 & 1.47 & 5640 & 1.55 & 2.55 & -2.64 & 56 & 0.36 & 0.06 & 0.06 \\
\hline HB & CS 22879-103 & 14.30 & 0.045 & 0.44 & 1.52 & 5720 & 1.60 & 2.55 & -2.15 & 44 & 0.22 & 0.06 & 0.05 \\
\hline SG & CS 22880-067 & 15.08 & 0.083 & 0.43 & 1.36 & 5570 & 3.35 & 1.05 & -3.37 & 57 & 0.20 & 0.08 & 0.07 \\
\hline RG & CS 22880-086 & 14.41 & 0.067 & 0.57 & 1.94 & 4960 & 1.90 & 1.40 & -3.25 & 39 & 0.15 & 0.06 & 0.06 \\
\hline SG & CS 22881-032 & 15.23 & 0.015 & 0.36 & 1.25 & 5430 & 3.20 & 1.25 & -3.10 & 36 & 0.18 & 0.06 & 0.06 \\
\hline SG & CS 22881-036 & 13.93 & 0.014 & 0.44 & 1.21 & 5940 & 3.70 & 1.10 & -2.37 & 45 & 0.16 & 0.06 & 0.06 \\
\hline HB & CS 22881-039 & 15.14 & 0.014 & 0.38 & 1.46 & 6170 & 2.05 & 2.80 & -2.79 & 40 & 0.29 & 0.06 & 0.06 \\
\hline SG & CS 22881-070 & 14.39 & 0.011 & 0.36 & 1.19 & 6020 & 3.70 & 1.35 & -2.73 & 49 & 0.19 & 0.06 & 0.07 \\
\hline HB & CS 22882-001 & 14.82 & 0.018 & 0.38 & 1.46 & 5930 & 1.90 & 3.00 & -2.62 & 52 & 0.32 & 0.06 & 0.05 \\
\hline SG & CS 22882-006 & 14.14 & 0.023 & 0.39 & 1.35 & 5800 & 3.60 & 1.35 & -2.66 & 44 & 0.18 & 0.06 & 0.07 \\
\hline SG & CS 22882-012 & 15.26 & 0.015 & 0.46 & 1.18 & 6290 & 3.80 & 1.40 & -2.75 & 48 & 0.17 & 0.06 & 0.07 \\
\hline SG & CS 22882-030 & 14.83 & 0.017 & 0.36 & 1.34 & 5580 & 3.40 & 1.10 & -3.14 & 45 & 0.14 & 0.06 & 0.06 \\
\hline HB & CS 22883-037 & 14.73 & 0.028 & 0.52 & 1.28 & 5800 & 1.50 & 3.15 & -1.97 & 44 & 0.25 & 0.06 & 0.05 \\
\hline SG & CS 22884-020 & 14.95 & 0.110 & 0.35 & 1.12 & 6040 & 3.75 & 1.45 & -2.27 & 49 & 0.19 & 0.06 & 0.06 \\
\hline SG & CS 22884-108 & 14.24 & 0.135 & 0.37 & 1.28 & 6320 & 3.75 & 1.20 & -3.21 & 52 & 0.17 & 0.06 & 0.10 \\
\hline SG & CS 22885-040 & 15.24 & 0.056 & 0.34 & 1.29 & 6160 & 3.75 & 1.45 & -2.60 & 47 & 0.17 & 0.06 & 0.06 \\
\hline RG & CS 22885-096 & 13.33 & 0.056 & 0.63 & 2.09 & 4580 & 0.75 & 1.75 & -4.21 & 34 & 0.15 & 0.06 & 0.07 \\
\hline SG & CS 22885-203 & 14.23 & 0.046 & 0.36 & 1.27 & 5820 & 3.60 & 1.30 & -2.57 & 44 & 0.16 & 0.06 & 0.06 \\
\hline SG & CS 22886-003 & 14.60 & 0.043 & 0.37 & 1.18 & 5970 & 3.65 & 1.40 & -2.87 & 38 & 0.16 & 0.06 & 0.09 \\
\hline SG & CS 22886-012 & 14.52 & 0.049 & 0.42 & 1.37 & 5650 & 3.50 & 1.45 & -2.61 & 42 & 0.15 & 0.06 & 0.06 \\
\hline HB & CS 22886-013 & 13.65 & 0.050 & 0.19 & 0.73 & 6200 & 1.75 & 2.95 & -2.60 & 40 & 0.22 & 0.06 & 0.06 \\
\hline SG & CS 22886-044 & 14.23 & 0.050 & 0.46 & 1.45 & 5730 & 3.75 & 1.20 & -1.65 & 43 & 0.19 & 0.06 & 0.06 \\
\hline SG & CS 22888-002 & 14.70 & 0.018 & 0.40 & 1.36 & 5700 & 3.50 & 1.30 & -2.91 & 46 & 0.15 & 0.06 & 0.07 \\
\hline SG & CS 22888-014 & 14.44 & 0.021 & 0.38 & 1.21 & 6020 & 3.70 & 1.35 & -2.69 & 45 & 0.19 & 0.06 & 0.06 \\
\hline SG & CS 22888-031 & 14.90 & 0.014 & 0.40 & 1.28 & 5810 & 3.50 & 1.25 & -3.41 & 44 & 0.20 & 0.06 & 0.08 \\
\hline HB & CS 22888-047 & 14.61 & 0.019 & 0.38 & 1.44 & 5950 & 1.90 & 3.00 & -2.54 & 46 & 0.27 & 0.06 & 0.05 \\
\hline SG & CS 22889-050 & 14.28 & 0.055 & 0.32 & 1.19 & 5920 & 3.60 & 1.40 & -2.84 & 50 & 0.19 & 0.06 & 0.06 \\
\hline SG & CS 22890-011 & 14.61 & 0.045 & 0.33 & 1.15 & 6010 & 3.70 & 1.25 & -2.47 & 57 & 0.18 & 0.06 & 0.06 \\
\hline
\end{tabular}


Table 7

(Continued)

\begin{tabular}{|c|c|c|c|c|c|c|c|c|c|c|c|c|c|}
\hline Class $^{\mathrm{a}}$ & Star & $V$ & $E(B-V)$ & $(B-V)_{0}$ & $(V-K)_{0}$ & $\begin{array}{l}T_{\text {eff }} \\
(\mathrm{K})\end{array}$ & $\log g$ & $\begin{array}{c}v_{\mathrm{t}} \\
\left(\mathrm{km} \mathrm{s}^{-1}\right)\end{array}$ & {$[\mathrm{M} / \mathrm{H}]$} & $\begin{array}{l}\sigma_{T_{\text {eff }}} \\
(\mathrm{K})\end{array}$ & $\sigma_{\log g}$ & $\begin{array}{c}\sigma_{v_{\mathrm{t}}} \\
\left(\mathrm{km} \mathrm{s}^{-1}\right)\end{array}$ & $\sigma_{[\mathrm{M} / \mathrm{H}]^{\mathrm{b}}}$ \\
\hline RG & CS 22890-024 & 13.41 & 0.054 & 0.52 & 1.82 & 5320 & 2.95 & 1.25 & -2.58 & 37 & 0.17 & 0.06 & 0.06 \\
\hline SG & CS 22890-064 & 14.70 & 0.043 & 0.35 & 1.16 & 5960 & 3.60 & 1.30 & -3.20 & 52 & 0.16 & 0.06 & 0.06 \\
\hline HB & CS 22891-184 & 13.83 & 0.071 & 0.43 & 1.45 & 5530 & 1.60 & 2.05 & -2.67 & 65 & 0.45 & 0.06 & 0.05 \\
\hline RG & CS 22891-200 & 13.93 & 0.079 & 0.78 & 2.30 & 4490 & 0.50 & 1.70 & -3.88 & 33 & 0.12 & 0.06 & 0.06 \\
\hline RG & CS 22891-209 & 12.17 & 0.078 & 0.75 & 2.33 & 4620 & 0.95 & 1.80 & -3.26 & 34 & 0.13 & 0.06 & 0.05 \\
\hline RG & CS 22891-221 & 14.43 & 0.076 & 0.48 & 1.62 & 5290 & 2.80 & 1.85 & -3.48 & 42 & 0.25 & 0.06 & 0.07 \\
\hline SG & CS 22892-025 & 14.03 & 0.032 & 0.36 & 1.21 & 6140 & 3.75 & 1.45 & -2.66 & 43 & 0.19 & 0.06 & 0.06 \\
\hline RG & CS 22892-052 & 13.21 & 0.031 & 0.77 & 2.19 & 4690 & 1.15 & 1.50 & -3.16 & 37 & 0.15 & 0.06 & 0.07 \\
\hline RG & CS 22893-005 & 14.22 & 0.041 & 0.49 & 1.66 & 5260 & 2.75 & 1.30 & -2.93 & 44 & 0.22 & 0.06 & 0.07 \\
\hline RG & CS 22893-010 & 14.74 & 0.038 & 0.55 & $\ldots$ & 5150 & 2.45 & 1.35 & -2.93 & 44 & 0.20 & 0.06 & 0.07 \\
\hline SG & CS 22893-011 & 14.54 & 0.041 & 0.39 & 1.29 & 6010 & 3.75 & 1.40 & -2.27 & 49 & 0.19 & 0.06 & 0.07 \\
\hline SG & CS 22894-004 & 14.17 & 0.043 & 0.37 & 1.25 & 5920 & 3.65 & 1.50 & -2.65 & 41 & 0.19 & 0.06 & 0.07 \\
\hline SG & CS 22894-019 & 13.92 & 0.035 & 0.41 & 1.32 & 5930 & 3.60 & 1.25 & -3.04 & 48 & 0.19 & 0.06 & 0.10 \\
\hline SG & CS 22894-023 & 13.75 & 0.035 & 0.37 & 1.27 & 5980 & 3.65 & 1.40 & -2.82 & 42 & 0.17 & 0.06 & 0.07 \\
\hline MS & CS 22894-049 & 14.46 & 0.030 & 0.42 & 1.35 & 5870 & 3.90 & 1.15 & -2.83 & 36 & 0.22 & 0.09 & 0.08 \\
\hline RG & CS 22896-015 & 14.85 & 0.058 & 0.35 & $\ldots$ & 5080 & 2.25 & 1.25 & -2.84 & 38 & 0.16 & 0.06 & 0.07 \\
\hline HB & CS 22896-055 & 13.53 & 0.050 & 0.38 & 1.44 & 5970 & 1.85 & 3.15 & -2.51 & 48 & 0.28 & 0.06 & 0.06 \\
\hline HB & CS 22896-110 & 13.56 & 0.060 & 0.47 & 1.62 & 5380 & 1.15 & 2.25 & -2.86 & 48 & 0.39 & 0.06 & 0.06 \\
\hline SG & CS 22896-115 & 14.13 & 0.058 & 0.36 & 1.27 & 5910 & 3.65 & 1.20 & -2.72 & 45 & 0.14 & 0.06 & 0.08 \\
\hline SG & CS 22896-136 & 14.71 & 0.059 & 0.35 & 1.14 & 6190 & 3.85 & 1.40 & -2.19 & 47 & 0.21 & 0.06 & 0.07 \\
\hline RG & CS 22897-008 & 13.33 & 0.033 & 0.66 & 2.24 & 4550 & 0.70 & 1.70 & -3.73 & 36 & 0.14 & 0.06 & 0.07 \\
\hline HB & CS 22898-043 & 14.06 & 0.050 & 0.38 & 1.28 & 6160 & 2.15 & 3.10 & -2.80 & 48 & 0.30 & 0.06 & 0.07 \\
\hline RG & CS 22898-047 & 14.24 & 0.059 & 0.50 & 1.74 & 5150 & 2.40 & 1.40 & -3.45 & 37 & 0.18 & 0.06 & 0.09 \\
\hline HB & CS 22937-072 & 14.02 & 0.041 & 0.49 & 1.69 & 5500 & 1.75 & 2.05 & -2.71 & 44 & 0.22 & 0.06 & 0.06 \\
\hline HB & CS 22940-070 & 14.87 & 0.055 & 0.42 & 1.41 & 6130 & 1.85 & 3.00 & -1.46 & 84 & 0.47 & 0.06 & 0.06 \\
\hline HB & CS 22940-077 & 14.13 & 0.070 & 0.46 & 1.72 & 5350 & 1.35 & 2.00 & -3.10 & 44 & 0.22 & 0.06 & 0.06 \\
\hline HB & CS 22940-121 & 14.16 & 0.053 & 0.50 & 1.75 & 5200 & 1.15 & 1.95 & -3.13 & 48 & 0.30 & 0.06 & 0.07 \\
\hline RG & CS 22941-017 & 15.00 & 0.021 & 0.54 & 1.84 & 5070 & 2.20 & 1.45 & -3.08 & 40 & 0.17 & 0.06 & 0.08 \\
\hline HB & CS 22941-027 & 14.05 & 0.016 & 0.33 & 1.26 & 6050 & 1.70 & 3.15 & -2.71 & 48 & 0.30 & 0.06 & 0.06 \\
\hline RG & CS 22942-002 & 13.79 & 0.021 & 0.59 & 1.77 & 5010 & 2.00 & 1.55 & -3.53 & 38 & 0.17 & 0.06 & 0.10 \\
\hline RG & CS 22942-011 & 12.94 & 0.017 & 0.61 & 2.01 & 4930 & 1.85 & 1.25 & -2.83 & 39 & 0.17 & 0.06 & 0.07 \\
\hline RG & CS 22942-035 & 14.56 & 0.021 & 0.59 & 1.96 & 4940 & 1.85 & 1.50 & -2.99 & 38 & 0.16 & 0.06 & 0.06 \\
\hline SG & CS 22943-059 & 14.40 & 0.041 & & 0.97 & 5810 & 3.60 & 1.15 & -2.64 & 54 & 0.15 & 0.06 & 0.07 \\
\hline SG & CS 22943-095 & 11.71 & 0.033 & 0.32 & 1.16 & 6140 & 3.80 & 1.35 & -2.44 & 51 & 0.18 & 0.06 & 0.07 \\
\hline SG & CS 22943-132 & 13.31 & 0.043 & 0.37 & 1.25 & 5850 & 3.60 & 1.40 & -2.67 & 41 & 0.16 & 0.06 & 0.08 \\
\hline RG & CS 22943-137 & 14.45 & 0.038 & 0.37 & 1.42 & 5400 & 3.10 & 1.20 & -3.39 & 48 & 0.27 & 0.06 & 0.08 \\
\hline HB & CS 22943-201 & 15.98 & 0.037 & 0.33 & 2.12 & 5970 & 2.45 & 1.60 & -2.69 & 52 & 0.39 & 0.06 & 0.06 \\
\hline SG & CS 22944-014 & 14.18 & 0.057 & 0.37 & 1.29 & 6020 & 3.70 & 1.30 & -2.62 & 45 & 0.20 & 0.06 & 0.08 \\
\hline RG & CS 22944-032 & 13.28 & 0.043 & 0.55 & 1.84 & 5090 & 2.30 & 1.45 & -3.15 & 36 & 0.14 & 0.06 & 0.08 \\
\hline SG & CS 22944-061 & 14.35 & 0.046 & 0.38 & 1.34 & 5920 & 3.60 & 1.50 & -2.95 & 45 & 0.21 & 0.06 & 0.08 \\
\hline SG & CS 22945-017 & 14.43 & 0.020 & 0.37 & 1.12 & 6080 & 3.70 & 1.25 & -2.73 & 54 & 0.19 & 0.06 & 0.08 \\
\hline RG & CS 22945-024 & 14.36 & 0.026 & 0.69 & 1.86 & 5120 & 2.35 & 1.15 & -2.59 & 50 & 0.24 & 0.07 & 0.06 \\
\hline RG & CS 22945-028 & 14.64 & 0.025 & 0.66 & 1.92 & 4900 & 1.75 & 1.50 & -2.89 & 41 & 0.18 & 0.06 & 0.06 \\
\hline SG & CS 22945-031 & 14.77 & 0.022 & 0.36 & 1.18 & 5820 & 3.55 & 1.35 & -3.03 & 47 & 0.19 & 0.06 & 0.08 \\
\hline HB & CS 22945-056 & 14.09 & 0.020 & 0.38 & 1.42 & 6000 & 1.75 & 3.45 & -2.83 & 48 & 0.25 & 0.06 & 0.06 \\
\hline SG & CS 22945-058 & 14.96 & 0.023 & 0.34 & 1.21 & 5990 & 3.65 & 1.55 & -2.71 & 45 & 0.17 & 0.06 & 0.08 \\
\hline HB & CS 22945-063 & 14.55 & 0.022 & $\ldots$ & 1.55 & 5730 & 1.70 & 2.55 & -2.84 & 48 & 0.31 & 0.06 & 0.06 \\
\hline HB & CS 22947-187 & 12.96 & 0.064 & 0.58 & 1.76 & 5300 & 1.40 & 1.85 & -2.58 & 52 & 0.37 & 0.06 & 0.06 \\
\hline RG & CS 22948-066 & 13.47 & 0.025 & 0.61 & 1.96 & 4830 & 1.55 & 2.00 & -3.18 & 34 & 0.15 & 0.06 & 0.07 \\
\hline SG & CS 22948-093 & 15.18 & 0.016 & 0.34 & 1.14 & 6540 & 3.85 & 1.15 & -3.42 & 51 & 0.15 & 0.06 & 0.13 \\
\hline SG & CS 22949-030 & 13.85 & 0.045 & 0.38 & 1.21 & 5890 & 3.60 & 1.40 & -2.88 & 48 & 0.16 & 0.06 & 0.07 \\
\hline RG & CS 22949-037 & 14.36 & 0.051 & 0.74 & 2.14 & 4630 & 0.95 & 1.70 & -4.20 & 34 & 0.13 & 0.06 & 0.07 \\
\hline RG & CS 22949-048 & 13.67 & 0.036 & 0.80 & 2.29 & 4620 & 0.95 & 1.70 & -3.37 & 35 & 0.14 & 0.06 & 0.07 \\
\hline RG & CS 22950-046 & 14.22 & 0.062 & & 2.46 & 4380 & 0.50 & 1.80 & -3.64 & 32 & 0.12 & 0.06 & 0.07 \\
\hline SG & CS 22951-005 & 14.90 & 0.017 & 0.37 & 1.15 & 5950 & 3.65 & 1.30 & -2.67 & 48 & 0.16 & 0.06 & 0.07 \\
\hline RG & CS 22951-059 & 14.44 & 0.017 & 0.54 & 1.70 & 5120 & 2.35 & 1.50 & -2.83 & 42 & 0.19 & 0.06 & 0.07 \\
\hline HB & CS 22951-077 & 13.61 & 0.016 & 0.48 & 1.72 & 5290 & 1.45 & 1.85 & -2.54 & 52 & 0.43 & 0.06 & 0.06 \\
\hline RG & CS 22952-015 & 13.27 & 0.035 & 0.78 & 2.26 & 4500 & 0.55 & 1.75 & -3.68 & 33 & 0.13 & 0.06 & 0.07 \\
\hline RG & CS 22953-003 & 13.77 & 0.017 & 0.67 & 2.08 & 4860 & 1.65 & 1.45 & -3.00 & 37 & 0.17 & 0.06 & 0.07 \\
\hline SG & CS 22953-037 & 13.64 & 0.028 & 0.34 & 1.11 & 6150 & 3.70 & 1.65 & -3.05 & 47 & 0.17 & 0.06 & 0.10 \\
\hline SG & CS 22954-004 & 14.27 & 0.028 & 0.39 & 1.26 & 5810 & 3.55 & 1.30 & -2.78 & 51 & 0.18 & 0.06 & 0.08 \\
\hline SG & CS 22954-015 & 13.03 & 0.031 & 0.42 & 1.23 & 5930 & 3.75 & 1.30 & -1.97 & 42 & 0.17 & 0.06 & 0.06 \\
\hline BS & CS 22955-110 & 13.61 & 0.052 & 0.29 & 0.92 & 6710 & 3.45 & 1.90 & -1.39 & 36 & 0.29 & 0.06 & 0.06 \\
\hline HB & CS 22955-174 & 14.38 & 0.049 & 0.45 & 1.48 & 5520 & 1.35 & 2.20 & -3.10 & 48 & 0.27 & 0.06 & 0.07 \\
\hline SG & CS 22956-015 & 14.19 & 0.042 & 0.35 & 1.21 & 5960 & 3.70 & 1.35 & -2.48 & 48 & 0.15 & 0.06 & 0.07 \\
\hline
\end{tabular}


Table 7

(Continued)

\begin{tabular}{|c|c|c|c|c|c|c|c|c|c|c|c|c|c|}
\hline Class $^{\mathrm{a}}$ & Star & $V$ & $E(B-V)$ & $(B-V)_{0}$ & $(V-K)_{0}$ & $\begin{array}{l}T_{\text {eff }} \\
(\mathrm{K})\end{array}$ & $\log g$ & $\begin{array}{c}v_{\mathrm{t}} \\
\left(\mathrm{km} \mathrm{s}^{-1}\right)\end{array}$ & {$[\mathrm{M} / \mathrm{H}]$} & $\begin{array}{l}\sigma_{T_{\text {eff }}} \\
(\mathrm{K})\end{array}$ & $\sigma_{\log g}$ & $\begin{array}{c}\sigma_{v_{\mathrm{t}}} \\
\left(\mathrm{km} \mathrm{s}^{-1}\right)\end{array}$ & $\sigma_{[\mathrm{M} / \mathrm{H}]^{\mathrm{b}}}$ \\
\hline SG & CS 22956-021 & 14.70 & 0.038 & 0.34 & 1.19 & 6020 & 3.70 & 1.20 & -2.51 & 49 & 0.18 & 0.06 & 0.08 \\
\hline RG & CS 22956-050 & 14.25 & 0.034 & 0.74 & 2.14 & 4640 & 1.00 & 1.75 & -3.57 & 36 & 0.14 & 0.06 & 0.07 \\
\hline SG & CS 22956-062 & 14.84 & 0.033 & 0.44 & 1.40 & 5540 & 3.40 & 1.65 & -2.76 & 42 & 0.15 & 0.06 & 0.07 \\
\hline SG & CS 22956-081 & 15.18 & 0.029 & 0.35 & 1.26 & 6310 & 3.80 & 1.65 & -2.80 & 57 & 0.20 & 0.06 & 0.07 \\
\hline SG & CS 22956-102 & 15.10 & 0.026 & 0.41 & 1.26 & 6220 & 3.85 & 1.50 & -2.21 & 60 & 0.23 & 0.06 & 0.07 \\
\hline SG & CS 22956-106 & 14.47 & 0.032 & 0.35 & 1.12 & 6410 & 3.90 & 1.75 & -2.60 & 53 & 0.21 & 0.06 & 0.07 \\
\hline HB & CS 22956-110 & 14.79 & 0.027 & 0.36 & 1.25 & 6010 & 1.85 & 2.85 & -3.14 & 56 & 0.39 & 0.06 & 0.07 \\
\hline RG & CS 22956-114 & 14.04 & 0.027 & 0.57 & $\ldots$ & 4900 & 1.75 & 1.85 & -2.86 & 39 & 0.16 & 0.06 & 0.06 \\
\hline RG & CS 22957-013 & 14.08 & 0.030 & 0.70 & 2.14 & 4620 & 0.95 & 1.55 & -3.01 & 35 & 0.15 & 0.06 & 0.06 \\
\hline SG & CS 22957-019 & 13.71 & 0.035 & 0.38 & 1.30 & 6070 & 3.75 & 1.25 & -2.42 & 42 & 0.19 & 0.06 & 0.07 \\
\hline RG & CS 22957-022 & 13.34 & 0.033 & 0.58 & 1.94 & 4860 & 1.65 & 1.55 & -3.17 & 37 & 0.15 & 0.06 & 0.07 \\
\hline SG & CS 22957-024 & 14.30 & 0.032 & 0.35 & 1.19 & 6160 & 3.75 & 1.35 & -2.81 & 55 & 0.20 & 0.06 & 0.09 \\
\hline SG & CS 22957-026 & 13.16 & 0.040 & 0.40 & 1.25 & 6120 & 3.90 & 1.35 & -1.89 & 47 & 0.25 & 0.06 & 0.07 \\
\hline RG & CS 22957-027 & 13.60 & 0.039 & 0.79 & 1.99 & 5220 & 2.65 & 1.45 & -3.00 & 39 & 0.23 & 0.06 & 0.07 \\
\hline SG & CS 22957-036 & 14.43 & 0.038 & 0.38 & 1.21 & 5970 & 3.75 & 1.25 & -2.09 & 45 & 0.19 & 0.06 & 0.06 \\
\hline HB & CS 22958-037 & 14.91 & 0.019 & 0.51 & 1.66 & 5770 & 2.10 & 2.30 & -2.51 & 40 & 0.29 & 0.06 & 0.06 \\
\hline SG & CS 22958-041 & 15.07 & 0.024 & 0.40 & 1.29 & 6010 & 3.65 & 1.55 & -2.88 & 39 & 0.30 & 0.06 & 0.08 \\
\hline SG & CS 22958-042 & 14.52 & 0.025 & 0.45 & 1.24 & 5760 & 3.55 & 0.95 & -2.99 & 57 & 0.18 & 0.08 & 0.11 \\
\hline SG & CS 22958-052 & 14.22 & 0.024 & 0.41 & 1.14 & 6090 & 3.75 & 1.95 & -2.42 & 46 & 0.18 & 0.06 & 0.07 \\
\hline SG & CS 22958-065 & 14.48 & 0.023 & 0.44 & 1.24 & 6020 & 3.75 & 1.30 & -2.24 & 57 & 0.20 & 0.06 & 0.07 \\
\hline SG & CS 22958-074 & 14.80 & 0.025 & 0.47 & 1.31 & 5800 & 3.60 & 1.40 & -2.62 & 44 & 0.18 & 0.06 & 0.08 \\
\hline RG & CS 22958-083 & 14.42 & 0.036 & 0.63 & 1.93 & 4900 & 1.75 & 1.40 & -3.04 & 35 & 0.13 & 0.06 & 0.07 \\
\hline RG & CS 22959-074 & 14.22 & 0.093 & 0.56 & 1.95 & 4940 & 1.90 & 1.35 & -2.49 & 35 & 0.17 & 0.06 & 0.06 \\
\hline SG & CS 22959-139 & 14.29 & 0.059 & 0.34 & 1.25 & 6090 & 3.75 & 1.55 & -2.45 & 46 & 0.17 & 0.06 & 0.07 \\
\hline MS & CS 22960-010 & 13.81 & 0.011 & 0.45 & 1.21 & 5280 & 4.00 & 1.20 & -3.03 & 44 & 0.36 & 0.13 & 0.08 \\
\hline SG & CS 22960-029 & 15.05 & 0.016 & 0.39 & 1.36 & 5890 & 3.60 & 1.45 & -2.77 & 52 & 0.14 & 0.06 & 0.08 \\
\hline RG & CS 22960-048 & 14.96 & 0.018 & 0.60 & 1.95 & 4770 & 1.40 & 1.55 & -3.78 & 36 & 0.14 & 0.06 & 0.07 \\
\hline RG & CS 22960-053 & 14.95 & 0.012 & 0.74 & 2.16 & 4860 & 1.65 & 1.60 & -3.33 & 40 & 0.18 & 0.06 & 0.08 \\
\hline RG & CS 22960-064 & 13.94 & 0.017 & 0.60 & 1.87 & 5060 & 2.20 & 1.40 & -2.77 & 36 & 0.14 & 0.06 & 0.07 \\
\hline RG & CS 22963-004 & 14.98 & 0.052 & 0.48 & 1.64 & 5060 & 2.15 & 1.50 & -3.85 & 42 & 0.16 & 0.06 & 0.08 \\
\hline SG & CS 22964-115 & 14.94 & 0.083 & 0.35 & 1.08 & 6090 & 3.70 & 1.40 & -2.70 & 54 & 0.15 & 0.06 & 0.07 \\
\hline SG & CS 22964-176 & 15.33 & 0.078 & 0.36 & 1.15 & 6000 & 3.70 & 1.40 & -2.46 & 49 & 0.19 & 0.06 & 0.07 \\
\hline SG & CS 22964-183 & 14.45 & 0.079 & 0.26 & 1.20 & 6010 & 3.65 & 1.45 & -2.87 & 42 & 0.15 & 0.06 & 0.08 \\
\hline SG & CS 22964-214 & 13.66 & 0.088 & 0.31 & 1.11 & 6180 & 3.75 & 1.45 & -2.84 & 47 & 0.17 & 0.06 & 0.08 \\
\hline RG & CS 22965-016 & 14.05 & 0.044 & 0.71 & 2.16 & 4770 & 1.40 & 1.85 & -2.93 & 33 & 0.12 & 0.06 & 0.06 \\
\hline SG & CS 22965-054 & 15.07 & 0.130 & 0.37 & 1.27 & 6050 & 3.70 & 1.60 & -2.89 & 53 & 0.15 & 0.06 & 0.07 \\
\hline SG & CS 22968-001 & 14.74 & 0.018 & 0.37 & 1.25 & 5940 & 3.65 & 1.30 & -2.84 & 52 & 0.21 & 0.06 & 0.09 \\
\hline RG & CS 22968-014 & 13.68 & 0.012 & 0.74 & 2.18 & 4540 & 0.60 & 1.75 & -3.85 & 34 & 0.14 & 0.06 & 0.08 \\
\hline SG & CS 22968-026 & 14.24 & 0.033 & 0.40 & 1.39 & 5850 & 3.65 & 1.20 & -2.33 & 44 & 0.18 & 0.06 & 0.07 \\
\hline SG & CS 22968-029 & 14.29 & 0.016 & 0.39 & 1.35 & 5760 & 3.50 & 1.40 & -3.11 & 47 & 0.15 & 0.06 & 0.09 \\
\hline SG & CS 29493-023 & 14.59 & 0.031 & 0.35 & 1.22 & 5980 & 3.65 & 1.25 & -2.66 & 52 & 0.15 & 0.06 & 0.08 \\
\hline SG & CS 29493-050 & 14.39 & 0.033 & 0.35 & 1.10 & 6270 & 3.80 & 1.45 & -2.89 & 48 & 0.17 & 0.06 & 0.08 \\
\hline SG & CS 29493-062 & 13.17 & 0.034 & 0.43 & 1.53 & 5520 & 3.40 & 1.15 & -2.59 & 44 & 0.18 & 0.06 & 0.08 \\
\hline SG & CS 29493-094 & 14.12 & 0.025 & 0.35 & 1.19 & 6130 & 3.80 & 1.55 & -2.44 & 43 & 0.19 & 0.06 & 0.07 \\
\hline SG & CS 29495-005 & 14.32 & 0.035 & 0.38 & 1.26 & 5990 & 3.75 & 1.15 & -2.26 & 38 & 0.19 & 0.06 & 0.08 \\
\hline RG & CS 29495-042 & 13.61 & 0.033 & 0.59 & 1.65 & 5180 & 2.55 & 1.45 & -2.18 & 46 & 0.26 & 0.06 & 0.06 \\
\hline BS & CS 29497-030 & 12.66 & 0.017 & 0.28 & 0.88 & 7000 & 4.00 & 1.60 & -2.52 & 44 & 0.39 & 0.06 & 0.08 \\
\hline RG & CS 29498-043 & 13.72 & 0.104 & 1.08 & 2.78 & 4440 & 0.50 & 1.75 & -3.85 & 20 & 0.13 & 0.06 & 0.08 \\
\hline SG & CS 29499-003 & 14.29 & 0.017 & 0.35 & 1.09 & 6080 & 3.75 & 1.25 & -2.35 & 46 & 0.18 & 0.06 & 0.07 \\
\hline SG & CS 29499-058 & 13.76 & 0.021 & 0.37 & 1.26 & 6060 & 3.70 & 1.45 & -2.61 & 42 & 0.17 & 0.06 & 0.08 \\
\hline SG & CS 29499-060 & 13.03 & 0.020 & 0.35 & 1.12 & 6280 & 3.80 & 1.30 & -2.75 & 52 & 0.18 & 0.06 & 0.09 \\
\hline SG & CS 29501-032 & 14.53 & 0.093 & 0.38 & 1.28 & 5950 & 3.65 & 0.60 & -2.71 & 48 & 0.14 & 0.07 & 0.09 \\
\hline RG & CS 29502-092 & 11.87 & 0.097 & 0.67 & 2.00 & 4820 & 1.50 & 1.50 & -3.20 & 34 & 0.14 & 0.06 & 0.07 \\
\hline HB & CS 29504-004 & 14.34 & 0.019 & 0.16 & 0.58 & 7500 & 2.50 & 1.95 & -2.35 & 44 & 0.18 & 0.06 & 0.06 \\
\hline SG & CS 29504-006 & 14.39 & 0.018 & 0.35 & 0.99 & 6150 & 3.70 & 1.60 & -2.91 & 68 & 0.22 & 0.06 & 0.07 \\
\hline SG & CS 29506-007 & 14.18 & 0.045 & 0.33 & 1.19 & 5940 & 3.60 & 1.50 & -2.94 & 42 & 0.15 & 0.06 & 0.08 \\
\hline SG & CS 29506-090 & 14.33 & 0.045 & 0.36 & 1.14 & 5940 & 3.60 & 1.35 & -3.08 & 42 & 0.20 & 0.06 & 0.10 \\
\hline BS & CS 29509-027 & 12.44 & 0.022 & 0.29 & 0.91 & 6850 & 3.50 & 1.65 & -2.42 & 41 & 0.22 & 0.06 & 0.07 \\
\hline SG & CS 29512-073 & 14.14 & 0.051 & 0.52 & 1.49 & 5650 & 3.60 & 0.90 & -1.93 & 52 & 0.23 & 0.07 & 0.07 \\
\hline HB & CS 29513-003 & 13.52 & 0.014 & 0.48 & 1.67 & 5480 & 1.90 & 1.75 & -2.46 & 48 & 0.34 & 0.06 & 0.06 \\
\hline HB & CS 29513-014 & 13.80 & 0.013 & 0.70 & 1.50 & 5440 & 1.55 & 2.00 & -2.32 & 52 & 0.38 & 0.06 & 0.06 \\
\hline SG & CS 29513-015 & 14.25 & 0.017 & 0.35 & 1.17 & 6110 & 3.75 & 1.35 & -2.51 & 46 & 0.16 & 0.06 & 0.07 \\
\hline SG & CS 29513-031 & 15.09 & 0.020 & 0.33 & 0.98 & 6600 & 4.00 & 0.75 & -2.59 & 61 & 0.28 & 0.07 & 0.08 \\
\hline SG & CS 29513-032 & 14.47 & 0.019 & 0.44 & 1.37 & 6080 & 3.85 & 1.30 & -1.91 & 50 & 0.21 & 0.06 & 0.06 \\
\hline SG & CS 29514-007 & 13.97 & 0.023 & 0.37 & 1.25 & 6400 & 3.85 & 1.75 & -2.81 & 45 & 0.19 & 0.06 & 0.07 \\
\hline
\end{tabular}


Table 7

(Continued)

\begin{tabular}{|c|c|c|c|c|c|c|c|c|c|c|c|c|c|}
\hline Class $^{\mathrm{a}}$ & Star & $V$ & $E(B-V)$ & $(B-V)_{0}$ & $(V-K)_{0}$ & $\begin{array}{l}T_{\text {eff }} \\
(\mathrm{K})\end{array}$ & $\log g$ & $\begin{array}{c}v_{\mathrm{t}} \\
\left(\mathrm{km} \mathrm{s}^{-1}\right)\end{array}$ & {$[\mathrm{M} / \mathrm{H}]$} & $\begin{array}{l}\sigma_{T_{\text {eff }}} \\
(\mathrm{K})\end{array}$ & $\sigma_{\log g}$ & $\begin{array}{c}\sigma_{v_{\mathrm{t}}} \\
\left(\mathrm{km} \mathrm{s}^{-1}\right)\end{array}$ & $\sigma_{[\mathrm{M} / \mathrm{H}]^{\mathrm{b}}}$ \\
\hline RG & CS 29514-017 & 13.55 & 0.015 & 0.62 & 1.62 & 5270 & 2.80 & 1.20 & -2.34 & 45 & 0.26 & 0.06 & 0.06 \\
\hline SG & CS 29514-018 & 13.34 & 0.017 & 0.48 & 1.17 & 5990 & 3.70 & 1.25 & -2.58 & 45 & 0.15 & 0.06 & 0.07 \\
\hline SG & CS 29514-037 & 13.95 & 0.014 & 0.49 & 1.22 & 5970 & 3.70 & 1.15 & -2.47 & 45 & 0.20 & 0.06 & 0.08 \\
\hline SG & CS 29517-018 & 14.50 & 0.025 & 0.41 & 1.39 & 5680 & 3.50 & 1.55 & -2.94 & 37 & 0.16 & 0.06 & 0.09 \\
\hline SG & CS 29517-042 & 14.10 & 0.026 & 0.36 & 1.20 & 6120 & 3.75 & 1.40 & -2.54 & 47 & 0.17 & 0.06 & 0.08 \\
\hline SG & CS 29529-054 & 14.85 & 0.039 & 0.49 & 1.52 & 5710 & 3.55 & 1.65 & -2.75 & 40 & 0.18 & 0.06 & 0.07 \\
\hline RG & CS 30312-044 & 13.34 & 0.083 & 0.70 & 2.12 & 4660 & 1.05 & 1.65 & -3.19 & 35 & 0.14 & 0.06 & 0.06 \\
\hline RG & CS 30312-059 & 13.10 & 0.114 & 0.69 & 2.09 & 4780 & 1.40 & 1.65 & -3.26 & 36 & 0.14 & 0.06 & 0.07 \\
\hline HB & CS 30312-062 & 12.58 & 0.119 & 0.21 & 0.95 & 6600 & 2.45 & 3.00 & -2.65 & 40 & 0.33 & 0.06 & 0.07 \\
\hline RG & CS 30312-100 & 12.92 & 0.083 & 0.63 & 1.81 & 5040 & 2.15 & 1.30 & -2.68 & 38 & 0.17 & 0.06 & 0.07 \\
\hline RG & CS 30314-067 & 11.85 & 0.065 & 1.13 & 2.82 & 4320 & 0.50 & 1.85 & -3.01 & 12 & 0.10 & 0.06 & 0.06 \\
\hline SG & CS 30339-015 & 15.28 & 0.017 & 0.38 & 1.27 & 5840 & 3.60 & 1.45 & -2.86 & 38 & 0.15 & 0.06 & 0.07 \\
\hline HB & CS 30339-046 & 15.31 & 0.016 & 0.37 & 1.20 & 7000 & 2.55 & 3.15 & -2.69 & 48 & 0.49 & 0.06 & 0.07 \\
\hline HB & CS 30339-052 & 15.30 & 0.012 & 0.48 & 1.70 & 5580 & 2.35 & 2.10 & -2.67 & 65 & 0.45 & 0.06 & 0.06 \\
\hline SG & CS 30339-069 & 14.74 & 0.009 & 0.15 & 1.27 & 5900 & 3.55 & 1.15 & -3.17 & 52 & 0.20 & 0.06 & 0.08 \\
\hline RG & CS 30339-073 & 14.75 & 0.012 & 0.53 & 1.95 & 4830 & 1.55 & 1.50 & -3.80 & 38 & 0.15 & 0.06 & 0.08 \\
\hline SG & CS 30492-001 & 14.20 & 0.031 & 0.42 & 1.96 & 5790 & 3.65 & 0.85 & -2.35 & 50 & 0.15 & 0.07 & 0.07 \\
\hline SG & CS 30492-016 & 13.89 & 0.033 & 0.42 & 1.40 & 5570 & 3.35 & 1.50 & -3.40 & 45 & 0.15 & 0.06 & 0.10 \\
\hline RG & CS 30492-110 & 14.63 & 0.088 & 0.67 & 2.13 & 4660 & 1.05 & 1.80 & -3.16 & 40 & 0.19 & 0.06 & 0.06 \\
\hline SG & CS 30493-071 & 13.21 & 0.021 & 0.40 & 1.38 & 5700 & 3.55 & 1.30 & -2.46 & 40 & 0.14 & 0.06 & 0.07 \\
\hline RG & CS 30494-003 & 12.33 & 0.051 & 0.63 & 1.80 & 4930 & 1.85 & 1.35 & -2.97 & 37 & 0.15 & 0.06 & 0.07 \\
\hline RG & CS 31082-001 & 11.67 & 0.016 & 0.75 & 2.16 & 4650 & 1.05 & 1.55 & -3.03 & 35 & 0.14 & 0.06 & 0.07 \\
\hline RG & BD-20 6008 & 9.84 & 0.043 & 0.83 & 2.32 & 4540 & 0.65 & 1.70 & -3.05 & 33 & 0.16 & 0.06 & 0.06 \\
\hline RG & BD-18 5550 & 9.25 & 0.165 & 0.75 & 2.24 & 4660 & 1.05 & 1.60 & -3.15 & 34 & 0.14 & 0.06 & 0.07 \\
\hline RG & BD-15 5781 & 10.72 & 0.041 & $\ldots$ & 2.32 & 4550 & 0.70 & 1.70 & -2.87 & 34 & 0.14 & 0.06 & 0.06 \\
\hline RG & BD-01 2582 & 9.66 & 0.022 & $\ldots$ & 2.00 & 4920 & 1.80 & 1.50 & -2.65 & 40 & 0.17 & 0.06 & 0.07 \\
\hline RG & BD+10 2495 & 9.72 & 0.022 & $\ldots$ & 2.09 & 4890 & 1.85 & 1.50 & -2.14 & 34 & 0.13 & 0.06 & 0.06 \\
\hline MS & $\mathrm{BD}+191185 \mathrm{~A}$ & 9.32 & 1.127 & $\ldots$ & $\ldots$ & 5440 & 4.30 & 1.05 & -1.25 & 41 & 0.24 & 0.12 & 0.06 \\
\hline SG & BD+24 1676 & 10.82 & 0.057 & $\ldots$ & 1.13 & 6140 & 3.75 & 1.45 & -2.54 & 43 & 0.18 & 0.06 & 0.08 \\
\hline SG & $\mathrm{BD}+263578$ & 9.36 & 0.671 & $\ldots$ & $\ldots$ & 6060 & 3.75 & 1.30 & -2.41 & 42 & 0.17 & 0.06 & 0.08 \\
\hline RG & BD+29 2356 & 11.35 & 0.013 & $\ldots$ & 2.26 & 4710 & 1.75 & 1.50 & -1.62 & 42 & 0.20 & 0.06 & 0.06 \\
\hline RG & $\mathrm{BD}+44493$ & 9.04 & 0.092 & $\ldots$ & 1.59 & 5040 & 2.10 & 1.35 & -4.26 & 36 & 0.14 & 0.06 & 0.12 \\
\hline RG & $\mathrm{BD}+80245^{\mathrm{c}}$ & 10.07 & 0.026 & 0.51 & 1.74 & 5360 & 3.15 & 1.20 & -2.01 & 34 & 0.22 & 0.06 & 0.07 \\
\hline RG & CD-38 245 & 11.97 & 0.012 & $\ldots$ & 2.29 & 4520 & 0.65 & 1.80 & -4.50 & 34 & 0.13 & 0.06 & 0.08 \\
\hline HB & CD-36 1052 & 9.94 & 0.025 & 0.44 & 1.36 & 6030 & 2.05 & 3.30 & -1.86 & 44 & 0.24 & 0.06 & 0.05 \\
\hline RG & CD-30 298 & 10.78 & 0.024 & $\ldots$ & 1.89 & 4810 & 1.50 & 1.55 & -3.72 & 38 & 0.15 & 0.06 & 0.07 \\
\hline RG & CD-24 1782 & 9.65 & 0.020 & 0.59 & 1.64 & 4950 & 1.90 & 1.50 & -3.02 & 36 & 0.14 & 0.06 & 0.06 \\
\hline SG & G004-036 & 11.49 & 0.099 & 0.38 & 1.21 & 5810 & 3.65 & 1.35 & -2.22 & 40 & 0.17 & 0.06 & 0.07 \\
\hline SG & G004-037 & 11.43 & 0.153 & 0.32 & 1.04 & 6110 & 3.75 & 1.35 & -2.67 & 46 & 0.18 & 0.06 & 0.08 \\
\hline MS & G009-027 & 12.11 & 0.022 & 0.57 & 1.74 & 5360 & 4.15 & 1.00 & -1.81 & 47 & 0.27 & 0.15 & 0.07 \\
\hline MS & G010-054 & 12.58 & 0.016 & 0.58 & 1.77 & 5210 & 4.40 & 0.80 & -2.30 & 41 & 0.53 & 0.12 & 0.08 \\
\hline MS & G015-010 & 12.06 & 0.088 & 0.56 & 1.68 & 5050 & 4.05 & 0.90 & -2.54 & 41 & 0.30 & 0.12 & 0.09 \\
\hline MS & G016-025 & 13.34 & 0.053 & 0.54 & 1.57 & 5250 & 4.10 & 1.10 & -2.07 & 53 & 0.31 & 0.17 & 0.09 \\
\hline MS & G025-022 & 12.32 & 0.088 & 0.47 & 1.38 & 5350 & 3.95 & 1.15 & -2.33 & 41 & 0.25 & 0.12 & 0.09 \\
\hline SG & G025-024 & 11.63 & 0.056 & 0.44 & 1.35 & 5670 & 3.55 & 1.70 & -2.28 & 45 & 0.15 & 0.06 & 0.08 \\
\hline SG & G026-001 & 11.27 & 0.062 & 0.43 & 1.33 & 5860 & 3.75 & 1.10 & -1.94 & 41 & 0.17 & 0.06 & 0.06 \\
\hline SG & G064-012 & 11.49 & 0.030 & 0.35 & 1.21 & 6030 & 3.60 & 1.20 & -3.39 & 36 & 0.18 & 0.06 & 0.10 \\
\hline SG & G071-055 & 10.78 & 0.026 & $\ldots$ & $\ldots$ & 5550 & 3.55 & 1.15 & -1.82 & 39 & 0.18 & 0.06 & 0.07 \\
\hline SG & G075-056 & 11.94 & 0.069 & 0.38 & 1.20 & 6190 & 3.85 & 1.45 & -2.30 & 51 & 0.20 & 0.06 & 0.09 \\
\hline MS & G088-023 & 12.04 & 0.056 & 0.43 & 1.34 & 5640 & 3.95 & 1.30 & -1.94 & 44 & 0.28 & 0.13 & 0.08 \\
\hline SG & G090-003 & 10.43 & 0.066 & 0.41 & 1.38 & 5680 & 3.60 & 1.20 & -2.24 & 37 & 0.15 & 0.06 & 0.07 \\
\hline MS & G090-025 & 8.30 & 0.050 & 0.56 & 1.63 & 5150 & 4.05 & 0.90 & -2.04 & 41 & 0.17 & 0.12 & 0.07 \\
\hline SG & G107-050 & 11.81 & 0.077 & 0.41 & 1.39 & 6030 & 3.85 & 1.20 & -1.89 & 49 & 0.20 & 0.06 & 0.08 \\
\hline MS & G115-049 & 11.60 & 0.022 & 0.48 & 1.45 & 5420 & 3.80 & 1.30 & -2.60 & 44 & 0.30 & 0.13 & 0.10 \\
\hline SG & G122-069 & 12.42 & 0.020 & 0.41 & 1.30 & 6010 & 3.70 & 1.65 & -2.50 & 42 & 0.22 & 0.06 & 0.09 \\
\hline SG & G126-062 & 9.48 & 0.053 & 0.39 & 1.26 & 5970 & 3.85 & 1.05 & -1.70 & 38 & 0.18 & 0.06 & 0.07 \\
\hline SG & G137-086 & 13.06 & 0.043 & 0.44 & 1.23 & 5820 & 3.75 & 1.15 & -1.76 & 40 & 0.16 & 0.06 & 0.07 \\
\hline MS & G146-056 & 13.24 & 0.017 & 0.61 & 1.72 & 5290 & 4.05 & 1.20 & -1.57 & 38 & 0.22 & 0.10 & 0.07 \\
\hline RG & G146-076 & 10.49 & 0.010 & 0.66 & 1.95 & 4980 & 2.10 & 1.30 & -2.13 & 37 & 0.17 & 0.06 & 0.07 \\
\hline MS & G153-064 & 11.44 & 0.237 & 0.46 & 1.34 & 5630 & 4.15 & 0.90 & -1.09 & 44 & 0.27 & 0.13 & 0.06 \\
\hline SG & G161-073 & 10.84 & 0.052 & 0.45 & 1.36 & 5680 & 3.90 & 1.05 & -1.07 & 49 & 0.22 & 0.06 & 0.06 \\
\hline SG & G166-047 & 12.04 & 0.016 & 0.39 & 1.29 & 5960 & 3.70 & 1.50 & -2.28 & 52 & 0.19 & 0.06 & 0.09 \\
\hline RG & G170-047 & 8.95 & 0.064 & 0.58 & 1.82 & 4930 & 1.85 & 1.50 & -2.90 & 35 & 0.12 & 0.06 & 0.07 \\
\hline MS & G180-058 & 11.31 & 0.010 & 0.68 & 1.89 & 4980 & 4.40 & 0.80 & -2.62 & 41 & 0.25 & 0.12 & 0.09 \\
\hline SG & G186-026 & 10.82 & 0.294 & 0.11 & 0.43 & 5950 & 3.65 & 1.45 & -2.90 & 45 & 0.15 & 0.06 & 0.08 \\
\hline
\end{tabular}


Table 7

(Continued)

\begin{tabular}{|c|c|c|c|c|c|c|c|c|c|c|c|c|c|}
\hline Class $^{\mathrm{a}}$ & Star & $V$ & $E(B-V)$ & $(B-V)_{0}$ & $(V-K)_{0}$ & $\begin{array}{l}T_{\text {eff }} \\
(\mathrm{K})\end{array}$ & $\log g$ & $\begin{array}{c}v_{\mathrm{t}} \\
\left(\mathrm{km} \mathrm{s}^{-1}\right)\end{array}$ & {$[\mathrm{M} / \mathrm{H}]$} & $\begin{array}{c}\sigma_{T_{\text {eff }}} \\
(\mathrm{K})\end{array}$ & $\sigma_{\log g}$ & $\begin{array}{c}\sigma_{v_{\mathrm{t}}} \\
\left(\mathrm{km} \mathrm{s}^{-1}\right)\end{array}$ & $\sigma_{[\mathrm{M} / \mathrm{H}]^{\mathrm{b}}}^{\mathrm{b}}$ \\
\hline SG & G188-022 ${ }^{\mathrm{c}}$ & 10.04 & 0.084 & 0.41 & 1.17 & 5890 & 3.90 & 0.95 & -1.44 & 48 & 0.22 & 0.06 & 0.07 \\
\hline MS & $\mathrm{G} 188-030^{\mathrm{c}}$ & 11.03 & 0.109 & 0.54 & 1.63 & 5090 & 4.30 & 0.85 & -2.07 & 44 & 0.20 & 0.13 & 0.07 \\
\hline MS & G190-010 & 11.22 & 0.237 & 0.37 & 1.15 & 5230 & 4.35 & 0.70 & -1.94 & 47 & 0.29 & 0.15 & 0.07 \\
\hline MS & G190-015 & 11.04 & 0.125 & 0.54 & 1.61 & 4950 & 3.85 & 1.50 & -3.13 & 41 & 0.40 & 0.12 & 0.09 \\
\hline SG & G199-066 & 12.68 & 0.018 & 0.48 & 1.46 & 5560 & 3.45 & 1.60 & -2.34 & 42 & 0.14 & 0.06 & 0.09 \\
\hline SG & G201-044 & 10.51 & 0.013 & 0.44 & 1.41 & 6000 & 3.90 & 1.05 & -1.63 & 42 & 0.19 & 0.06 & 0.06 \\
\hline SG & G206-034 & 11.40 & 0.155 & 0.28 & 0.94 & 5930 & 3.60 & 1.35 & -3.13 & 38 & 0.16 & 0.06 & 0.09 \\
\hline MS & G214-001 & 12.08 & 0.137 & 0.43 & 1.31 & 5370 & 4.05 & 0.95 & -2.29 & 44 & 0.23 & 0.13 & 0.09 \\
\hline SG & G234-028 & 11.10 & 0.040 & 0.44 & 1.34 & 5870 & 3.75 & 1.20 & -1.78 & 41 & 0.18 & 0.06 & 0.07 \\
\hline SG & G238-030 & 12.91 & 0.022 & 0.45 & 1.38 & 5740 & 3.50 & 1.65 & -3.40 & 53 & 0.14 & 0.06 & 0.12 \\
\hline MS & G238-032 & 12.66 & 0.016 & 0.74 & 1.67 & 5380 & 4.35 & 0.75 & -1.58 & 47 & 0.28 & 0.15 & 0.08 \\
\hline RG & HD 6268 & 8.08 & 0.017 & 0.84 & 2.32 & 4570 & 0.70 & 1.85 & -2.69 & 34 & 0.16 & 0.06 & 0.06 \\
\hline RG & HD 11582 & 9.57 & 0.016 & 0.64 & 1.88 & 5020 & 2.20 & 1.45 & -2.03 & 40 & 0.17 & 0.06 & 0.07 \\
\hline RG & HD 13979 & 9.18 & 0.014 & $\ldots$ & 2.05 & 4830 & 1.60 & 1.60 & -2.72 & 38 & 0.16 & 0.06 & 0.07 \\
\hline SG & HD $16031^{c}$ & 9.77 & 0.022 & 0.42 & 1.26 & 5870 & 3.75 & 0.90 & -1.91 & 38 & 0.15 & 0.06 & 0.07 \\
\hline SG & HD 19445 & 8.08 & 0.148 & 0.30 & 1.04 & 5820 & 3.65 & 1.25 & -2.40 & 38 & 0.15 & 0.06 & 0.07 \\
\hline RG & HD 21581 & 8.72 & 0.123 & 0.63 & 1.97 & 4940 & 2.10 & 1.50 & -1.82 & 40 & 0.20 & 0.06 & 0.06 \\
\hline RG & HD 26169 & 8.76 & 0.077 & 0.58 & 1.91 & 4750 & 1.35 & 1.60 & -2.81 & 34 & 0.12 & 0.06 & 0.06 \\
\hline RG & HD 26297 & 7.47 & 0.032 & 1.05 & 2.74 & 4400 & 1.10 & 1.75 & -1.72 & 38 & 0.26 & 0.06 & 0.06 \\
\hline SG & HD $31128^{c}$ & 9.14 & 0.037 & 0.45 & 1.31 & 5630 & 3.60 & 1.10 & -1.92 & 42 & 0.16 & 0.06 & 0.06 \\
\hline RG & HD 45282 & 8.02 & 0.567 & $\ldots$ & $\ldots$ & 5230 & 2.90 & 1.35 & -1.73 & 41 & 0.25 & 0.06 & 0.06 \\
\hline RG & HD 88609 & 8.57 & 0.009 & 0.93 & 2.54 & 4430 & 0.50 & 1.70 & -3.08 & 35 & 0.16 & 0.06 & 0.07 \\
\hline SG & HD $94028^{c}$ & 8.22 & 0.027 & 0.44 & 1.32 & 5730 & 3.70 & 1.00 & -1.81 & 43 & 0.20 & 0.06 & 0.07 \\
\hline $\mathrm{HB}$ & HD 106373 & 8.92 & 0.063 & $\ldots$ & 1.31 & 6040 & 1.85 & 2.90 & -2.60 & 40 & 0.29 & 0.06 & 0.07 \\
\hline RG & HD $108317^{c}$ & 8.05 & 0.018 & $\ldots$ & 1.85 & 5030 & 2.10 & 1.45 & -2.60 & 38 & 0.16 & 0.06 & 0.07 \\
\hline HB & HD 119516 & 9.09 & 0.032 & $\ldots$ & 1.64 & 5660 & 1.90 & 1.90 & -1.93 & 40 & 0.29 & 0.06 & 0.06 \\
\hline SG & HD $122196^{c}$ & 8.73 & 0.092 & 0.37 & 1.21 & 5880 & 3.80 & 1.20 & -1.79 & 45 & 0.22 & 0.06 & 0.06 \\
\hline RG & HD $122563^{c}$ & 6.20 & 0.025 & 0.88 & 2.39 & 4500 & 0.55 & 1.95 & -2.93 & 34 & 0.15 & 0.06 & 0.07 \\
\hline RG & HD $126238^{c}$ & 7.66 & 0.115 & 0.69 & 2.00 & 4750 & 1.65 & 1.55 & -1.96 & 37 & 0.23 & 0.06 & 0.06 \\
\hline RG & HD 126587 & 9.12 & 0.100 & 0.73 & 2.18 & 4640 & 1.00 & 1.75 & -3.21 & 34 & 0.13 & 0.06 & 0.07 \\
\hline RG & HD $128279^{c}$ & 8.02 & 0.100 & 0.54 & 1.68 & 5050 & 2.15 & 1.55 & -2.64 & 38 & 0.16 & 0.06 & 0.07 \\
\hline SG & HD $132475^{c}$ & 8.55 & 0.107 & 0.44 & 1.35 & 5410 & 3.50 & 1.00 & -1.65 & 40 & 0.25 & 0.06 & 0.07 \\
\hline RG & HD 175305 & 7.18 & 0.105 & 0.64 & 1.83 & 4920 & 2.30 & 1.40 & -1.56 & 42 & 0.22 & 0.06 & 0.06 \\
\hline SG & HD 175606 & 9.78 & 0.063 & 0.38 & 1.23 & 5920 & 3.70 & 1.20 & -2.24 & 45 & 0.18 & 0.06 & 0.07 \\
\hline $\mathrm{HB}$ & HD 178443 & 9.98 & 0.090 & 0.63 & 1.82 & 5170 & 1.45 & 1.85 & -2.02 & 48 & 0.32 & 0.06 & 0.06 \\
\hline $\mathrm{HB}$ & HD 184266 & 7.60 & 0.128 & 0.45 & 1.42 & 5780 & 1.85 & 2.55 & -1.74 & 44 & 0.24 & 0.06 & 0.06 \\
\hline RG & HD 186478 & 9.15 & 0.114 & 0.86 & 2.39 & 4540 & 0.65 & 1.75 & -2.78 & 34 & 0.14 & 0.06 & 0.06 \\
\hline MS & HD 188510 & 8.82 & 0.217 & $\ldots$ & $\ldots$ & 5210 & 4.10 & 0.80 & -1.88 & 44 & 0.27 & 0.13 & 0.07 \\
\hline MS & HD 193901 & 8.65 & 0.060 & 0.49 & 1.35 & 5580 & 4.05 & 1.10 & -1.28 & 47 & 0.24 & 0.15 & 0.06 \\
\hline SG & HD 196892 & 8.24 & 0.044 & 0.46 & 1.30 & 5670 & 3.85 & 1.05 & -1.24 & 49 & 0.17 & 0.06 & 0.06 \\
\hline HB & HD 196944 & 8.38 & 0.042 & $\ldots$ & 1.74 & 5310 & 1.75 & 1.65 & -2.39 & 48 & 0.33 & 0.06 & 0.06 \\
\hline RG & HD 200654 & 9.08 & 0.029 & 0.61 & 1.85 & 5080 & 2.25 & 1.35 & -3.06 & 36 & 0.14 & 0.06 & 0.08 \\
\hline MS & HD 201891 & 7.37 & 0.111 & 0.40 & 1.14 & 5840 & 4.10 & 1.10 & -1.21 & 41 & 0.32 & 0.12 & 0.07 \\
\hline HB & HD 214362 & 9.09 & 0.027 & 0.47 & 1.48 & 5760 & 1.60 & 2.25 & -2.04 & 48 & 0.26 & 0.06 & 0.06 \\
\hline SG & HD 219617 & 8.16 & 0.033 & 0.44 & 1.30 & 5730 & 3.70 & 1.30 & -1.83 & 37 & 0.16 & 0.06 & 0.07 \\
\hline RG & HD 220127 & 10.15 & 0.012 & 0.65 & 1.93 & 5060 & 2.40 & 1.30 & -1.85 & 40 & 0.19 & 0.06 & 0.07 \\
\hline RG & HD 237846 & 9.96 & 0.011 & $\ldots$ & 2.11 & 4730 & 1.30 & 1.45 & -3.14 & 34 & 0.13 & 0.06 & 0.07 \\
\hline RG & HE $0454-4758$ & 13.48 & 0.010 & 0.56 & 1.78 & 5140 & 2.40 & 1.35 & -3.32 & 40 & 0.18 & 0.06 & 0.08 \\
\hline SG & HE $0938+0114$ & 10.21 & 0.101 & 0.17 & 0.67 & 6030 & 3.65 & 1.20 & -2.92 & 42 & 0.17 & 0.06 & 0.10 \\
\hline RG & HE $1012-1540$ & 14.04 & 0.070 & $\ldots$ & 1.72 & 5230 & 2.65 & 1.70 & -3.76 & 32 & 0.20 & 0.06 & 0.14 \\
\hline RG & HE $1124-2335$ & 14.63 & 0.047 & 0.65 & 2.08 & 4870 & 1.65 & 1.45 & -3.26 & 23 & 0.14 & 0.06 & 0.07 \\
\hline RG & HE $1320-1339$ & 10.26 & 0.066 & 1.01 & 1.70 & 4690 & 1.20 & 1.75 & -2.93 & 34 & 0.14 & 0.06 & 0.06 \\
\hline SG & HIP 99423 & 8.88 & 0.577 & $\ldots$ & $\ldots$ & 5520 & 3.70 & 1.05 & -1.42 & 41 & 0.20 & 0.06 & 0.07 \\
\hline
\end{tabular}

Notes.

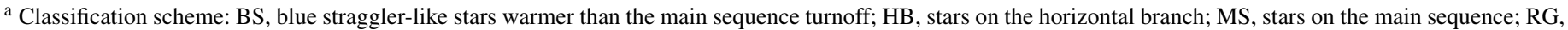
stars on the red giant branch; SG, stars on the subgiant branch.

${ }^{\mathrm{b}} \sigma_{[\mathrm{M} / \mathrm{H}]}=\sigma_{\mathrm{Fe} \mathrm{I}}$.

${ }^{c}$ Star with reliable parallax and $E(B-V)<0.12$.

(e.g., EW > $160 \mathrm{~m} \AA$ near $4000 \AA$ ). In many stars the strongest optical Fe I lines used have $\log (\mathrm{EW} / \lambda)>-4.5(\mathrm{EW}>130 \mathrm{~m} \AA$ near $4000 \AA$ ), which is on the saturated portion of the curve of growth in both dwarfs and giants. The weakest $\mathrm{Fe}_{\mathrm{I}}$ lines used often have $\log (\mathrm{EW} / \lambda)<-6.0(\mathrm{EW}<4 \mathrm{~m} \AA$ near $4000 \AA)$, but the weakest lines detected are a strong function of $\mathrm{S} / \mathrm{N}$. 
Table 8

Atomic Data

\begin{tabular}{|c|c|c|c|c|}
\hline $\begin{array}{l}\lambda \\
(\AA)\end{array}$ & Species & $\begin{array}{l}\text { E.P. } \\
(\mathrm{eV})\end{array}$ & $\log g f$ & Ref. \\
\hline \multirow[t]{4}{*}{6707.80} & Li I & 0.00 & +0.17 & 1 \\
\hline & \multirow{3}{*}{\multicolumn{3}{|c|}{$\begin{array}{c}\mathrm{CH} A^{2} \Delta-X^{2} \Pi G \text { band } \\
\text { NH } A^{3} \Pi-X^{3} \Sigma \text { band } \\
\text { CN } B^{2} \Sigma-X^{2} \Sigma \text { band }\end{array}$}} & 2 \\
\hline & & & & 3 \\
\hline & & & & 3 \\
\hline 6300.30 & [O I] & 0.00 & -9.78 & 4 \\
\hline 7771.94 & $\mathrm{O}_{\mathrm{I}}$ & 9.15 & +0.37 & 4 \\
\hline 7774.17 & $\mathrm{O}_{\mathrm{I}}$ & 9.15 & +0.22 & 4 \\
\hline 7775.39 & $\mathrm{O}_{\mathrm{I}}$ & 9.15 & +0.00 & 4 \\
\hline
\end{tabular}

References. (1) Smith et al. 1998; (2) B. Plez (2007, private communication); (3) Kurucz \& Bell 1995; (4) Fuhr \& Wiese 2009; (5) Chang \& Tang 1990; (6) Aldenius et al. 2007; (7) Aldenius et al. 2009; (8) Lawler \& Dakin 1989, using hfs from Kurucz \& Bell 1995; (9) Lawler et al. 2013; (10) Wood et al. 2013; (11) Pickering et al. 2001, with corrections given in Pickering et al. 2002; (12) Doerr et al. 1985, using hfs from Kurucz \& Bell 1995; (13) Biémont et al. 1989; (14) Sobeck et al. 2007; (15) Nilsson et al. 2006; (16) Den Hartog et al. 2011 for both $\log (g f)$ value and hfs; (17) O'Brian et al. 1991; (18) Meléndez \& Barbuy 2009; (19) Cardon et al. 1982, using hfs from Kurucz \& Bell 1995; (20) Nitz et al. 1999, using hfs from Kurucz \& Bell 1995; (21) Fuhr \& Wiese 2009, using hfs from Kurucz \& Bell 1995; (22) Roederer \& Lawler 2012; (23) Biémont et al. 2011; (24) Ljung et al. 2006; (25) Nilsson et al. 2010; (26) Whaling \& Brault 1988; (27) Palmeri et al. 2005; (28) Wickliffe et al. 1994; (29) Fuhr \& Wiese 2009, using hfs/IS from McWilliam 1998 when available; (30) Lawler et al. 2001a, using hfs from Ivans et al. 2006; (31) Lawler et al. 2009; (32) Li et al. 2007, using hfs from Sneden et al. 2009; (33) Den Hartog et al. 2003, using hfs/IS from Roederer et al. 2008b when available; (34) Lawler et al. 2006, using hfs/IS from Roederer et al. 2008b when available; (35) Lawler et al. 2001c, using hfs/ IS from Ivans et al. 2006; (36) Den Hartog et al. 2006; (37) Lawler et al. 2001b, using hfs from Lawler et al. 2001d; (38) Wickliffe et al. 2000; (39) Lawler et al. 2004 for both $\log (g f)$ value and hfs; (40) Lawler et al. 2008; (41) Wickliffe \& Lawler 1997; (42) Sneden et al. 2009 for both $\log (g f)$ value and hfs/IS; (43) Lawler et al. 2007; (44) Ivarsson et al. 2003, using hfs/IS from Cowan et al. 2005; (45) Biémont et al. 2000, using hfs/IS from Roederer et al. 2012; (46) Nilsson et al. 2002.

(This table is available in its entirety in machine-readable and Virtual Observatory (VO) forms in the online journal. A portion is shown here for guidance regarding its form and content.)

Overionization is the name given to the effect that occurs when the local mean radiation intensity exceeds that predicted by the Planck source function, and overionization leads to a potentially significant underestimate of the number density of minority species. This non-LTE effect can lead to systematic underestimates of the Fe I number density when using the LTE approximation for the source function in metal-poor stars (e.g., Thévenin \& Idiart 1999, Asplund 2005). To reduce the impact of this effect on the ionization balance between Fe I and Fe II, in most stars we derive surface gravities ( $\log g$, in cgs units) from theoretical isochrones in the $\mathrm{Y}^{2}$ grid (Demarque et al. 2004). For stars between the main sequence turnoff (MSTO) and the tip of the red giant branch, we interpolate the $\mathrm{Y}^{2}$ isochrones in $T_{\text {eff }}$ for the appropriate metallicity of each star. We assume an input age of 12 Gyr for all stars. Stars with ages $12.0 \pm$ $1.5 \mathrm{Gyr}$ were formed at redshifts $z>2$, so this range includes the ages of most metal-poor Milky Way globular clusters (e.g., Dotter et al. 2010), the ages of individual halo stars as computed from radioactive decay of heavy elements (e.g., Roederer et al. 2009), and the redshifts of the rising inferred star formation rate density in galaxies that may grow to the size of the Milky Way (e.g., Bouwens et al. 2007). For stars on the HB, below the isochrone inflection point (i.e., the main sequence; MS), or hotter than the MSTO, we derive surface gravities from the usual method of requiring the ionization balance between Fe I and Fe II. Uncertainties inherent to each of these methods are discussed below.

$\mathrm{Fe}$ II is the dominant ionization state, so overionization has little impact on the iron abundance derived from Fe II lines in stars of types F-G-K. We assume that the overall metallicity, $[\mathrm{M} / \mathrm{H}]$, is represented by the iron abundance derived from Fe II lines. The trade off, of course, is that the iron abundance derived from Fe II lines is more sensitive to the surface gravity of the model atmosphere than the iron abundance derived from $\mathrm{Fe}$ I lines.

We use the following procedure to converge to a final set of model parameters. Broadband photometry is used only to produce an initial estimate of $T_{\text {eff }}$ from dereddened $V-K$ or $J-K$ colors and the Alonso et al. (1999b) color $-T_{\text {eff }}$ relations. We have had to extrapolate the Alonso et al. calibrations to stars with $[\mathrm{Fe} / \mathrm{H}]<-3$ in our sample, but the metallicity sensitivity of such relations decreases with decreasing metallicity and these extrapolations are used for initial guesses only. Other relations, e.g., those of González Hernández \& Bonifacio (2009), are calibrated directly to the 2MASS photometric system and are valid at $[\mathrm{Fe} / \mathrm{H}]<-3$. These relations are used less frequently in prior abundance surveys, so we adopt the Alonso et al. relations for the sake of comparison. We estimate the initial $\log g$ by interpolating (in $V-K$ or $J-K$ ) the appropriate $\mathrm{Y}^{2}$ isochrones. We estimate the initial $v_{\mathrm{t}}$ from $\log g$ using the relation of Gratton et al. (1996). We compile metallicity estimates from many of the literature sources listed above, but they have relatively little impact on the initial estimates of the other parameters.

We use a recent version (c. 2010) of the spectral line analysis code MOOG $^{4}$ (Sneden 1973) to derive abundances of iron from Fe I and Fe II lines using our EW measurements and a MARCS model atmosphere interpolated for our initial model atmosphere parameters. We refine our initial $T_{\text {eff }}, v_{\mathrm{t}}$, and $[\mathrm{Fe} / \mathrm{H}]$ estimates to enforce no trend of derived iron abundance (from $\mathrm{Fe}$ I) with each line's E.P. or $\log (\mathrm{EW} / \lambda)$ value, and we require that the input model metallicity agrees with the iron abundance derived from Fe II lines. We maintain $\log g$ fixed through these iterations. Then, for stars along the subgiant branch and red giant branch, the revised $T_{\text {eff }}$ and $[\mathrm{Fe} / \mathrm{H}]$ estimates are used to refine $\log g$ from the isochrones. For stars on the HB, MS, or warmer than the MSTO, iron ionization equilibrium is enforced to derive $\log g$. Lines whose inferred abundance deviates by more than $2 \sigma$ from the mean are discarded. We repeat this process until all four parameters converge, and this usually occurs within two to four iterations. We consider convergence to mean that the model atmosphere parameters are stable from one iteration to the next to the nearest $10 \mathrm{~K}$ in $T_{\text {eff }}, 0.05$ dex in $\log g, 0.05 \mathrm{~km} \mathrm{~s}^{-1}$ in $v_{\mathrm{t}}$, and 0.01 dex in metallicity. We perform this iteration scheme using a version of the batch mode capabilities of MOOG, and all steps are supervised by the user.

Table 7 lists the final $T_{\text {eff }}, \log g, v_{\mathrm{t}}$, and $[\mathrm{Fe} / \mathrm{H}]$ for all 313 stars in the survey. Figure 6 illustrates these values. Different colors represent different metallicity ranges. The size of each point corresponds to the size of the microturbulence velocity parameter. Open circles represent stars whose gravity is derived from Fe ionization balance, while filled circles represent stars whose gravity is derived from the $\mathrm{Y}^{2}$ grid of isochrones. This approach leads to the narrow width of the subgiant and red giant branches and the non-physical gap between the red giant branch

\footnotetext{
4 http://www.as.utexas.edu/ chris/moog.html
} 


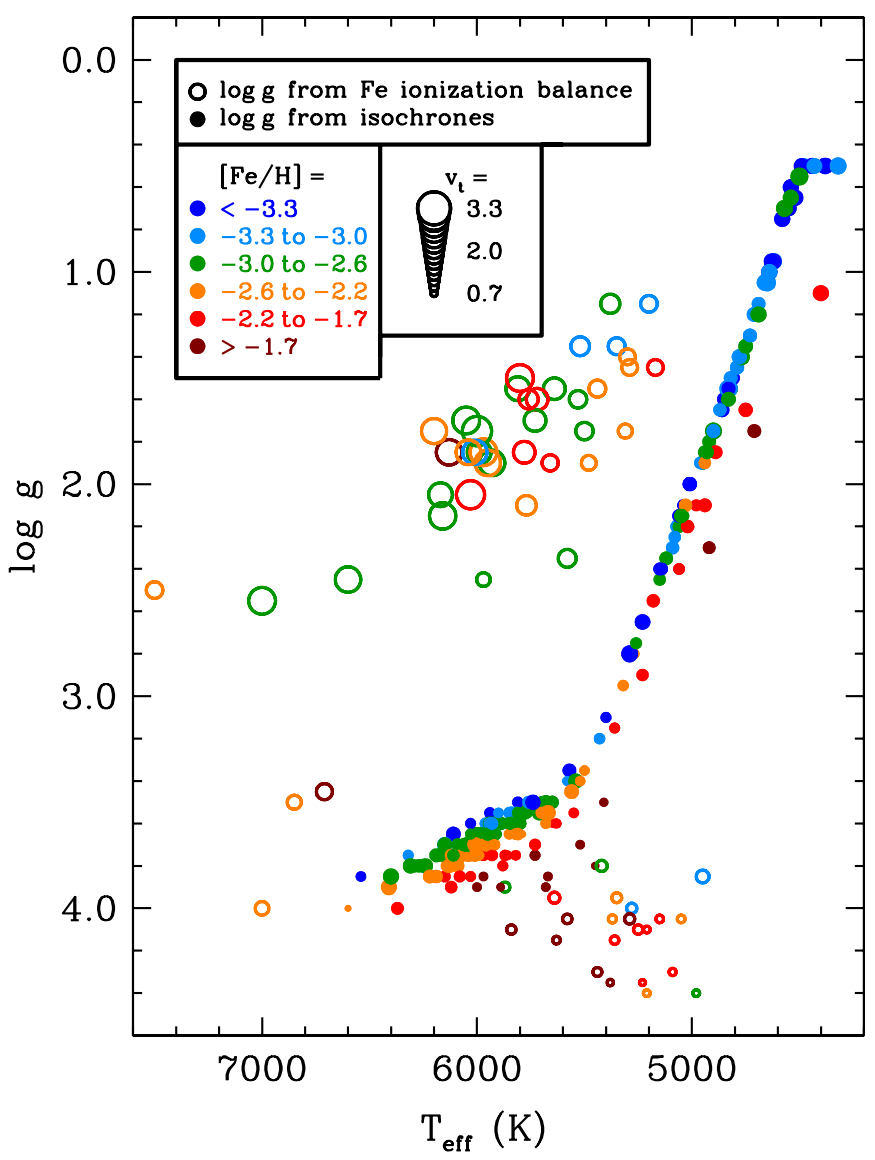

Figure 6. $T_{\text {eff }}$ vs. $\log g$ diagram for all 313 stars in our sample. Color-coding indicates the metallicity (from Fe II), and the point size indicates $v_{\mathrm{t}}$. Open circles represent stars whose $\log g$ was derived from Fe ionization balance, and closed circles represent stars whose $\log g$ was calculated from isochrones. The "bend" observed for few stars at the top of the red giant branch is a result of encountering the edge of the grid of model atmospheres at $\log g=0.5$ at low metallicity.

(A color version of this figure is available in the online journal.)

and the HB. The latter effect is reminiscent of the difference between absolute magnitudes of field red giants and the fiducial of globular cluster M92 found in Figure 4 of Luck \& Bond (1985). The bulk of the stars in our sample are subgiants (151 stars, denoted "SG" in Table 7) or red giants (98 stars, denoted "RG"). Smaller numbers of stars are on the HB (39 stars, denoted "HB"), MS (22 stars, denoted "MS"), or are warmer than the MSTO (3 stars, denoted "BS" in analogy with the blue straggler stars found in globular clusters).

\subsection{Statistical Uncertainties}

We use the statistical (internal) uncertainty in the derived $\mathrm{Fe}$ I and Fe II abundances ( $\sigma_{\mathrm{Fe}}$ and $\sigma_{\mathrm{Fe} \text { II }}$, respectively) to assess the statistical uncertainties in the model parameters. We relate the sensitivity of $T_{\text {eff }}$ and $v_{\mathrm{t}}$ to their correlation with E.P. and $\log (\mathrm{EW} / \lambda)$ through the statistical uncertainty in $\sigma_{\mathrm{Fe} \text { I }}$. Our tests for several stars spanning the range of the SG and RG classes suggest that simple linear relations with $T_{\text {eff }}$ and $v_{\mathrm{t}}$ are appropriate and lead to the relations $\sigma_{T_{\text {eff }}}^{2}=\left(-0.17 T_{\text {eff }}+\right.$ $540)^{2} \times \sigma_{\mathrm{Fe} \text { I }}^{2}$ and $\sigma_{v_{\mathrm{t}}}^{2}=\left(0.32 v_{\mathrm{t}}-0.90\right)^{2} \times \sigma_{\mathrm{FeI}}^{2}$. For other stars in our sample we adopt relationships that are independent of $T_{\text {eff }}$ and $v_{\mathrm{t}}$. For stars on the MS, we find $\sigma_{T_{\text {eff }}}^{2}=\left(400 \sigma_{\mathrm{Fe} \mathrm{I}}\right)^{2}$ and $\sigma_{v_{\mathrm{t}}}^{2}=\left(1.5 \sigma_{\mathrm{Fe}}\right)^{2}$. For stars on the HB, we find $\sigma_{T_{\mathrm{eff}}}^{2}=\left(520 \sigma_{\mathrm{Fe}}\right)^{2}$

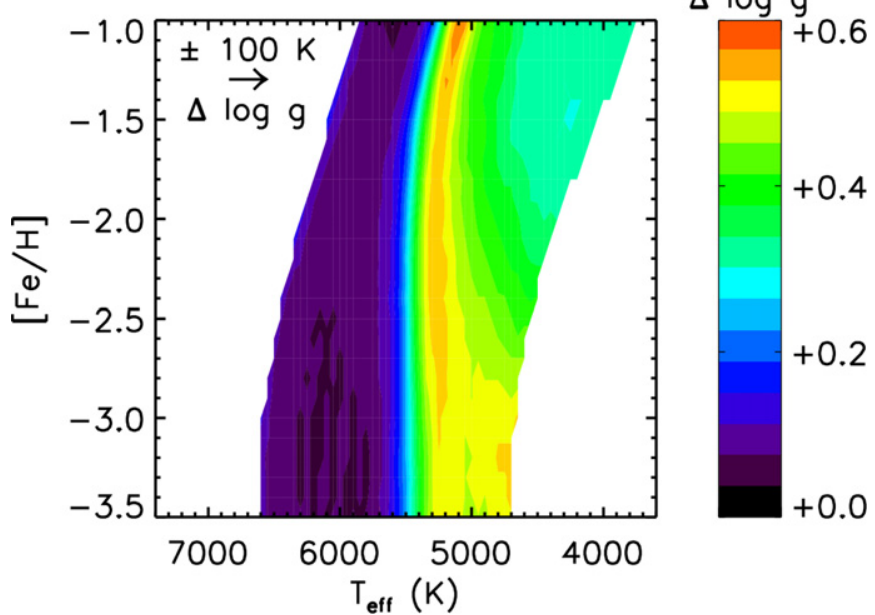

Figure 7. Variations in $\log g$ from the $\mathrm{Y}^{2}$ grid of isochrones corresponding to uncertainties in $T_{\text {eff }}$. At given values of $T_{\text {eff }}$ and $[\mathrm{Fe} / \mathrm{H}]$, the corresponding uncertainty in $\log g$ can be read off from the plot. The color bar on the right indicates the magnitude of the uncertainty in $\log g$ for a change in $T_{\text {eff }}$ of $100 \mathrm{~K}$. (A color version of this figure is available in the online journal.)

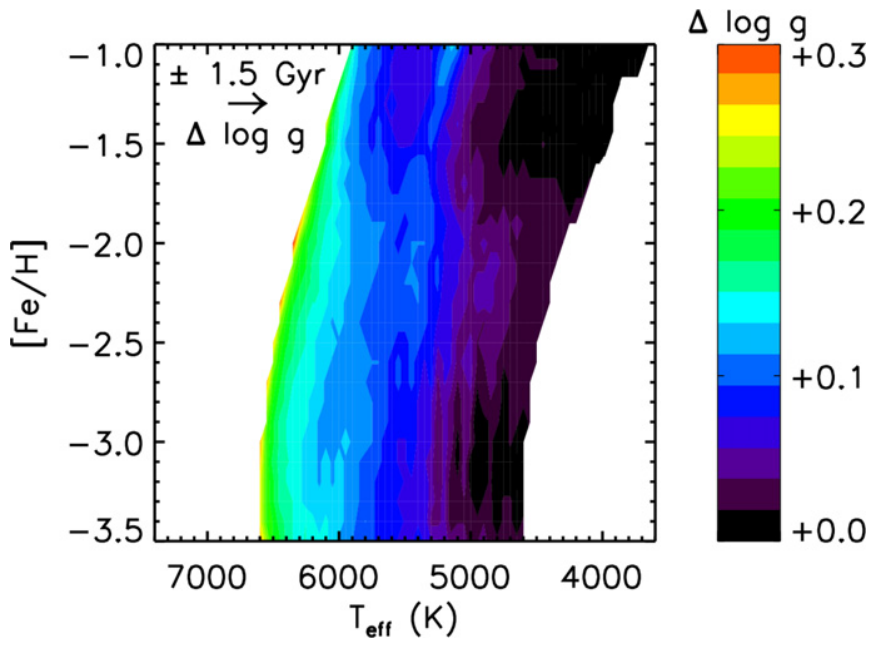

Figure 8. Variations in $\log g$ from the $\mathrm{Y}^{2}$ grid of isochrones corresponding to uncertainties in age. At given values of $T_{\text {eff }}$ and $[\mathrm{Fe} / \mathrm{H}]$, the corresponding uncertainty in $\log g$ can be read off from the plot. The color bar on the right indicates the magnitude of the uncertainty in $\log g$ if the isochrone age is varied by 1.5 Gyr (relative to the 12 Gyr isochrones).

(A color version of this figure is available in the online journal.)

and $\sigma_{v_{\mathrm{t}}}^{2}=\left(0.36 \sigma_{\mathrm{Fe}}\right)^{2}$. For stars warmer than the MSTO (BS), we find $\sigma_{T_{\text {eff }}}^{2}=\left(400 \sigma_{\mathrm{Fe}}\right)^{2}$ and $\sigma_{v_{\mathrm{t}}}^{2}=\left(0.48 \sigma_{\mathrm{Fe}}\right)^{2}$.

For stars in the $\mathrm{SG}$ and $\mathrm{RG}$ classes, we assess statistical uncertainties in $\log g$ by varying $T_{\text {eff }}$ and $[\mathrm{Fe} / \mathrm{H}]$ as input parameters to the grid of $\mathrm{Y}^{2}$ isochrones. In Figure 7 we illustrate the change in $\log g$ when $T_{\text {eff }}$ is varied by $\pm 100 \mathrm{~K}$. Stars along the giant branch are most sensitive because of its steep slope, with $\Delta \log g$ approaching 0.50 per $100 \mathrm{~K}$, whereas stars in the SG class and near the MSTO show $\Delta \log g$ ranging from only 0.05 to 0.20 per $100 \mathrm{~K}$. Figures 8 and 9 illustrate the change in $\log g$ when the isochrone age is varied by $\pm 1.5 \mathrm{Gyr}$, our assumed age uncertainty, or the input metallicity is varied by \pm 0.10 dex. (Recall that we assume an age of 12 Gyr in the isochrones.) The gravity is most sensitive to age near the MSTO, whereas stars on the red giant branch have almost no age sensitivity in old stellar populations. The gravity is most 


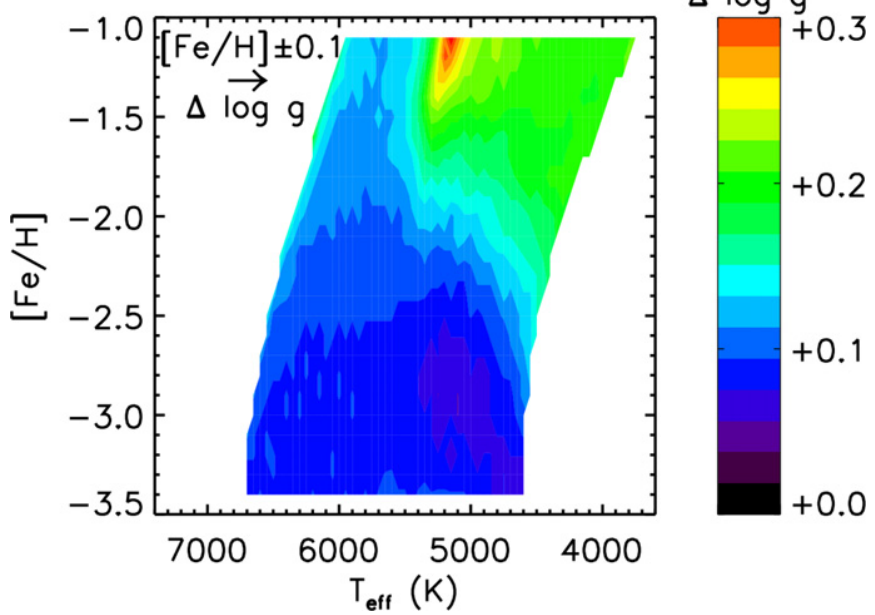

Figure 9. Variations in $\log g$ from the $\mathrm{Y}^{2}$ grid of isochrones corresponding to uncertainties in metallicity. At given values of $T_{\text {eff }}$ and $[\mathrm{Fe} / \mathrm{H}]$, the corresponding uncertainty in $\log g$ can be read off from the plot. The color bar on the right indicates the magnitude of the uncertainty in $\log g$ for a change in $[\mathrm{Fe} / \mathrm{H}]$ of 0.1 dex.

(A color version of this figure is available in the online journal.)

sensitive to the isochrone metallicity in more metal-rich stars where line blanketing has a significant impact on the temperature and color. For most of the stars in our sample, the uncertainty in $\log g$ resulting from the uncertainty in metallicity is very small relative to other sources of uncertainty. For stars on the MS, HB, and warmer than the MSTO, we assess the statistical uncertainty in $\log g$ by varying the gravity such that the iron abundances derived from Fe I and Fe II remain in agreement within $\pm\left(\sigma_{\mathrm{Fe} \text { I }}^{2}+\sigma_{\mathrm{Fe} \text { II }}^{2}\right)^{1 / 2}$. We consider statistical uncertainties in $[\mathrm{M} / \mathrm{H}]$ equal to the standard deviation of $\mathrm{Fe} \mathrm{I}$, since that species is used in deriving $v_{\mathrm{t}}$, its uncertainties, and the cross terms discussed in Section 9.1. The statistical uncertainties in $\log g$ listed in Table 7 include the uncertainty from each of these factors. All statistical uncertainties include a "softening" factor to guard against unreasonably small uncertainties (10 K in $T_{\mathrm{eff}}$; 0.05 dex in $\log g ; 0.05 \mathrm{~km} \mathrm{~s}^{-1}$ in $v_{\mathrm{t}}$; and $0.02 \mathrm{dex}$ in $\left.[\mathrm{M} / \mathrm{H}]\right)$.

To empirically test the sensitivity of the derived model parameters to the choice of model grid, we select two subsets of stars from the full sample and rederive model parameters using the ATLAS9 grid of $\alpha$-enhanced models with convective overshooting turned off. We begin the derivation from the original (unculled) set of EW measurements for each of these stars and follow the same set of procedures. One subset of stars is comprised of 32 subgiants with $5800 \mathrm{~K} \leqslant T_{\text {eff }} \leqslant$ $6000 \mathrm{~K}$ (as derived from the MARCS grid) and $-3.5 \leqslant[\mathrm{Fe} / \mathrm{H}]$ $\leqslant-2.5$. The other subset is comprised of 22 giants with $4790 \mathrm{~K}$ $\leqslant T_{\text {eff }} \leqslant 5090 \mathrm{~K}$ (as derived from the MARCS grid) and $-3.5 \leqslant$ $[\mathrm{Fe} / \mathrm{H}] \leqslant-2.5$. The model parameters derived for the subset of subgiants are nearly identical, with $\Delta T_{\text {eff }}=-8 \mathrm{~K}(\sigma=29 \mathrm{~K})$, $\Delta \log g=-0.005(\sigma=0.027), \Delta v_{\mathrm{t}}=+0.002 \mathrm{~km} \mathrm{~s}^{-1}(\sigma=$ $\left.0.039 \mathrm{~km} \mathrm{~s}^{-1}\right)$, and $\Delta[\mathrm{Fe} / \mathrm{H}]=-0.006 \mathrm{dex}(\sigma=0.023 \mathrm{dex})$. Differences are in the sense of MARCS minus ATLAS9. The model parameters derived for the subset of giants are similar but not identical, with $\Delta T_{\text {eff }}=+39 \mathrm{~K}(\sigma=26 \mathrm{~K}), \Delta \log g=+0.11$ $(\sigma=0.08), \Delta v_{\mathrm{t}}=-0.07 \mathrm{~km} \mathrm{~s}^{-1}\left(\sigma=0.03 \mathrm{~km} \mathrm{~s}^{-1}\right)$, and $\Delta[\mathrm{Fe} / \mathrm{H}]=+0.04 \mathrm{dex}(\sigma=0.03 \mathrm{dex})$. Thus, the ATLAS 9 grid tends to move stars slightly up the red giant branch to cooler temperatures, lower gravities, higher microturbulence velocities, and lower metallicities. Fortunately, the magnitude of the effect is rather small. The standard deviations derived in each of these tests also provide an estimate of the dispersion in model parameters that could be expected in the convergence routine. These uncertainties are comparable to the size of the statistical uncertainties. We include a model convergence uncertainty of $30 \mathrm{~K}$ in $T_{\text {eff }}, 0.08 \mathrm{dex}$ in $\log g$, and $0.04 \mathrm{~km} \mathrm{~s}^{-1}$ in $v_{\mathrm{t}}$ in the statistical uncertainties listed in Table 7.

\subsection{Comparison with Previous Surveys}

We compare our model atmosphere parameters with those determined by studies from the last $30 \mathrm{yr}$ that analyzed at least six stars in common with us: Luck \& Bond (1985), McWilliam et al. (1995a), Ryan et al. (1996), Fulbright (2000), Johnson (2002), Stephens \& Boesgaard (2002), Gratton et al. (2003), Cayrel et al. (2004), Honda et al. (2004b), Preston et al. (2006a), Lai et al. (2008), Bonifacio et al. (2009), Ishigaki et al. (2010), and Roederer et al. (2010). These studies can be divided into two general categories based on the primary means by which $T_{\text {eff }}$ has been determined. One group (group "b" in Table 9) uses the usual spectroscopic technique of determining $T_{\text {eff }}$ by minimizing the dependence of Fe I abundance with E.P., as we have done. The other group (group "a" in Table 9) relies on color $-T_{\text {eff }}$ relations or fits to the wings of Balmer series lines to calculate $T_{\text {eff }}$. Some of these studies use a hybrid of these techniques, and we have made our best attempt to divide them into one of the two categories for purposes of this comparison.

The differences and standard deviations in $T_{\text {eff }}, \log g, v_{\mathrm{t}}$, and metallicity (expressed as $[\mathrm{Fe} \mathrm{II} / \mathrm{H}]$; i.e., the iron abundance as derived from Fe II lines) are listed in Table 9 and illustrated in Figure 10. As has long been known (e.g., Luck \& Bond 1985), photometric $T_{\text {eff }}$ determinations consistently predict warmer temperatures and higher gravities than purely spectroscopic $T_{\text {eff }}$ predictions. The values listed in Table 9 reaffirm this situation.

There are no significant temperature differences between our study and previous ones that used the abundance versus E.P. approach, with a mean $\Delta T_{\text {eff }}=-28 \mathrm{~K}(\sigma=161 \mathrm{~K})$ from 80 stars. Differences in the gravities $(\Delta \log g=-0.24, \sigma=$ $0.41)$, microturbulence parameters $\left(\Delta v_{\mathrm{t}}=-0.11 \mathrm{~km} \mathrm{~s}^{-1}, \sigma=\right.$ $\left.0.36 \mathrm{~km} \mathrm{~s}^{-1}\right)$, and derived metallicities $(\Delta[\mathrm{Fe} / \mathrm{H}]=-0.13$, $\sigma=0.22 \mathrm{dex}$ ) are negative but not statistically significant. These offsets show only slight variations if the stellar evolutionary status is considered.

We find significant differences in the derived stellar parameters when we compare with studies that compute $T_{\text {eff }}$ by other methods. For the 110 stars in common, our temperatures are cooler $\left(\Delta T_{\text {eff }}=-185 \mathrm{~K}, \sigma=154 \mathrm{~K}\right)$, our gravities are lower $(\Delta \log g=-0.57, \sigma=0.42)$, and our microturbulence parameters are smaller $\left(\Delta v_{\mathrm{t}}=-0.26 \mathrm{~km} \mathrm{~s}^{-1}, \sigma=0.35 \mathrm{~km} \mathrm{~s}^{-1}\right)$. These offsets conspire to lower our derived mean metallicities by $0.21 \mathrm{dex}(\sigma=0.18 \mathrm{dex})$. These offsets show some dependence on evolutionary state. The most pronounced offsets are in red giants, where our mean metallicities are lower by 0.27 dex $(\sigma=0.17 \mathrm{dex})$. Except for $v_{\mathrm{t}}$, these differences are moderately significant.

Given the present state of modeling of the line-forming regions of stellar atmospheres, it is unclear whether one approach is preferable to another. Both are likely inadequate at some level. Abundance studies often adopt a technique based on the information available about the stellar sample or the quality of the spectra themselves (reliable photometry, knowledge of stellar distance, interstellar reddening, spectral coverage, etc.). Most studies of stars with $[\mathrm{Fe} / \mathrm{H}]<-2.5$ selected from BPS92 have adopted temperatures calculated from color $-T_{\text {eff }}$ relations 
Table 9

Comparison of Atmospheric Parameters with Previous Surveys

\begin{tabular}{|c|c|c|c|c|c|}
\hline Comparison & $N$ & $\begin{array}{c}\Delta T_{\text {eff }}(\sigma) \\
\quad(\mathrm{K})\end{array}$ & $\Delta \log g(\sigma)$ & $\begin{array}{c}\Delta v_{\mathrm{t}}(\sigma) \\
\left(\mathrm{km} \mathrm{s}^{-1}\right)\end{array}$ & $\Delta \mathrm{Fe}$ II $(\sigma)$ \\
\hline Luck \& Bond (1985) ${ }^{\mathrm{b}}$ & 11 & $-169(140)$ & $-0.17(0.44)$ & $-0.57(0.46)$ & $-0.49(0.18)$ \\
\hline McWilliam et al. (1995a) ${ }^{a}$ & 26 & $-174(117)$ & $-0.44(0.50)$ & $-0.56(0.26)$ & $-0.25(0.15)$ \\
\hline Ryan et al. $(1996)^{\mathrm{a}}$ & 10 & $-233(203)$ & $-0.82(0.41)$ & $-0.22(0.41)$ & $-0.38(0.20)$ \\
\hline Fulbright $(2000)^{\mathrm{b}}$ & 17 & $-43(140)$ & $-0.33(0.33)$ & $-0.08(0.28)$ & $-0.14(0.12)$ \\
\hline Johnson $(2002)^{\mathrm{b}}$ & 6 & $+12(45)$ & $-0.12(0.26)$ & $-0.27(0.28)$ & $-0.16(0.06)$ \\
\hline Stephens \& Boesgaard (2002) ${ }^{b}$ & 6 & $-44(218)$ & $-0.17(0.16)$ & $+0.18(0.37)$ & $-0.01(0.19)$ \\
\hline Gratton et al. $(2003)^{\mathrm{a}}$ & 6 & $-210(98)$ & $-0.41(0.24)$ & $+0.00(0.22)$ & $-0.10(0.15)$ \\
\hline Cayrel et al. (2004) ${ }^{\mathrm{a}}$ & 18 & $-236(104)$ & $-0.68(0.41)$ & $-0.22(0.14)$ & $-0.20(0.13)$ \\
\hline Honda et al. $(2004 b)^{a}$ & 10 & $-192(130)$ & $-0.76(0.29)$ & $-0.20(0.31)$ & $-0.31(0.23)$ \\
\hline Simmerer et al. $(2004)^{\mathrm{a}}$ & 19 & $-82(155)$ & $-0.47(0.35)$ & $-0.29(0.43)$ & $-0.22(0.17)$ \\
\hline Preston et al. $(2006 a)^{b}$ & 19 & $-58(124)$ & $-0.59(0.28)$ & $-0.02(0.27)$ & $-0.15(0.12)$ \\
\hline Lai et al. $(2008)^{\mathrm{a}}$ & 12 & $-233(204)$ & $-0.50(0.39)$ & $-0.10(0.08)$ & $-0.08(0.18)$ \\
\hline Bonifacio et al. (2009) ${ }^{\mathrm{a}}$ & 9 & $-184(198)$ & $-0.56(0.43)$ & $+0.07(0.30)$ & $-0.09(0.13)$ \\
\hline Ishigaki et al. $(2010)^{\mathrm{b}}$ & 10 & $+86(164)$ & $-0.13(0.31)$ & $+0.00(0.35)$ & $+0.00(0.20)$ \\
\hline Roederer et al. $(2010)^{\mathrm{b}}$ & 11 & $+75(163)$ & $+0.24(0.35)$ & $-0.04(0.14)$ & $+0.10(0.16)$ \\
\hline \multicolumn{6}{|c|}{ All stars } \\
\hline All & 190 & $-118(175)$ & $-0.43(0.44)$ & $-0.20(0.36)$ & $-0.18(0.20)$ \\
\hline Group (a) & 110 & $-185(154)$ & $-0.57(0.42)$ & $-0.26(0.35)$ & $-0.21(0.18)$ \\
\hline Group (b) & 80 & $-28(161)$ & $-0.24(0.41)$ & $-0.11(0.36)$ & $-0.13(0.22)$ \\
\hline \multicolumn{6}{|c|}{ Stars in class "RG" } \\
\hline All & 108 & $-154(160)$ & $-0.48(0.49)$ & $-0.30(0.35)$ & $-0.25(0.20)$ \\
\hline Group (a) & 73 & $-215(125)$ & $-0.65(0.43)$ & $-0.33(0.31)$ & $-0.27(0.17)$ \\
\hline Group (b) & 35 & $-27(151)$ & $-0.13(0.40)$ & $-0.23(0.41)$ & $-0.21(0.24)$ \\
\hline \multicolumn{6}{|c|}{ Stars in class "SG" } \\
\hline All & 40 & $-94(211)$ & $-0.30(0.37)$ & $-0.04(0.38)$ & $-0.04(0.18)$ \\
\hline Group (a) & 24 & $-144(199)$ & $-0.41(0.36)$ & $-0.08(0.41)$ & $-0.06(0.15)$ \\
\hline Group (b) & 16 & $-18(211)$ & $-0.14(0.34)$ & $+0.01(0.33)$ & $+0.01(0.22)$ \\
\hline \multicolumn{6}{|c|}{ Stars in class "HB" } \\
\hline All & 28 & $-7(160)$ & $-0.47(0.42)$ & $-0.11(0.31)$ & $-0.12(0.17)$ \\
\hline Group (a) & 5 & +44 (189) & $-0.52(0.47)$ & $-0.38(0.37)$ & $-0.17(0.18)$ \\
\hline Group (b) & 23 & $-19(156)$ & $-0.46(0.42)$ & $-0.05(0.26)$ & $-0.10(0.16)$ \\
\hline \multicolumn{6}{|c|}{ Stars in class "MS" } \\
\hline All & 14 & $-138(99)$ & $-0.30(0.19)$ & $-0.05(0.25)$ & $-0.15(0.08)$ \\
\hline Group (a) & 8 & $-172(108)$ & $-0.29(0.21)$ & $-0.09(0.22)$ & $-0.14(0.10)$ \\
\hline Group (b) & 6 & $-92(71)$ & $-0.31(0.16)$ & $+0.01(0.30)$ & $-0.15(0.07)$ \\
\hline
\end{tabular}

Notes. Differences are in the sense of this study minus other study. Quantities in parenthesis refer to the standard deviation.

a Studies that do not use Fe I abundance versus E.P. as the primary means of determining $T_{\text {eff }}$.

b Studies that use Fe I abundance versus E.P. as the primary means of determining $T_{\text {eff }}$.

or Balmer line profiles (e.g., McWilliam et al. 1995a, Ryan et al. 1996, Cayrel et al. 2004, Lai et al. 2008, Bonifacio et al. 2009), and so on average our derived metallicities will always be lower than theirs. Nevertheless, despite these differences, Figure 10 demonstrates that the relative metallicities are in good agreement for all but the most metal-poor giants. We discuss this point in more detail in Section 8.5.

Lind et al. (2012) have considered how line-by-line departures from LTE on Fe I and Fe II lines can act to influence the derivation of stellar parameters. For all parameter ranges included in our sample, and for the choice of the hydrogen collision parameter adopted by Lind et al., their study finds that LTE calculations of the Fe II abundance reflect that of non-LTE calculations to within 0.02 dex. This principle underlies our decision to adopt the iron abundance derived from Fe II lines as our metallicity indicator. For the metallicity range of our sample, Lind et al. also suggest that temperatures derived by our method will underestimate $T_{\text {eff }}$ by less than $30 \mathrm{~K}$ for red giants, overestimate $T_{\text {eff }}$ by less than $30 \mathrm{~K}$ for stars on the subgiant branch and MSTO, overestimate $T_{\text {eff }}$ by $40-120 \mathrm{~K}$ for stars on the $\mathrm{HB}$, and overestimate $T_{\text {eff }}$ by less than $20 \mathrm{~K}$ for stars on the MS. In summary, it seems unlikely that departures from LTE alone can account for the differences between the photometric and spectroscopic temperatures estimated for our stellar sample.

As illustrated in Figure 11, our derived $v_{\mathrm{t}}$ values for the most luminous giants are slightly lower that our initial guesses calculated from the relation given by Gratton et al. (1996). The Gratton et al. relation is shown by the dotted line. The solid line shows a linear least-squares fit to the giants in our sample, given by $v_{\mathrm{t}}=-0.20 \log g+1.88(\sigma=0.13)$. For less luminous giants, the two relations agree well. Despite the shallower slope, $\log g$ and $v_{\mathrm{t}}$ show a tight correlation for the giants in our sample. The difference for the most luminous giants can be attributed mostly to the lower $\log g$ values derived by our methods. Shifting the $\log g$ values for our RG stars an average of 0.65 dex to the right (Table 9, group "a") would bring the $v_{\mathrm{t}}$ values for 

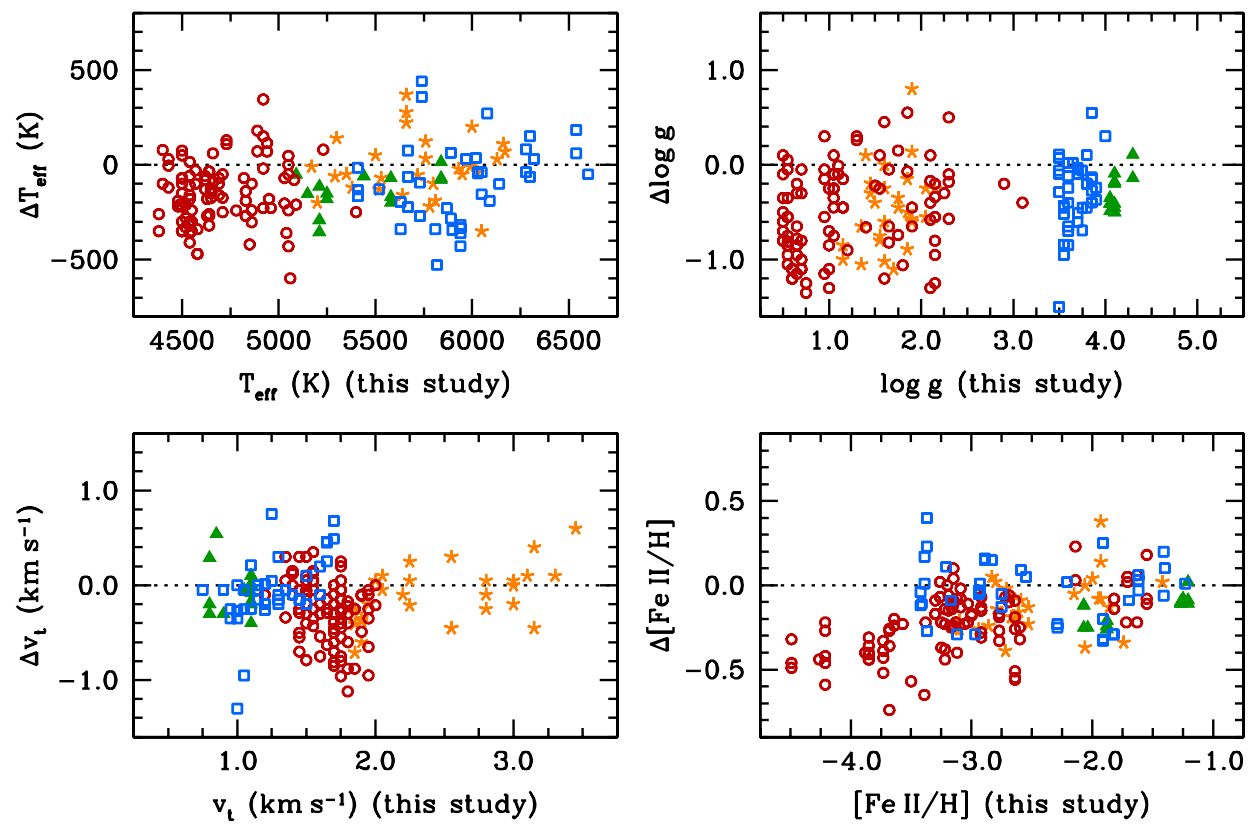

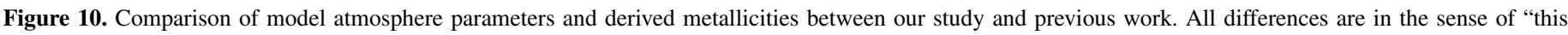

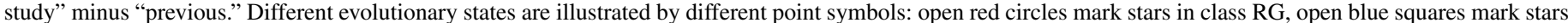
in class SG, orange starred symbols mark stars in class HB, and filled green triangles mark stars in class MS. The dotted lines indicate a difference of zero.

(A color version of this figure is available in the online journal.)

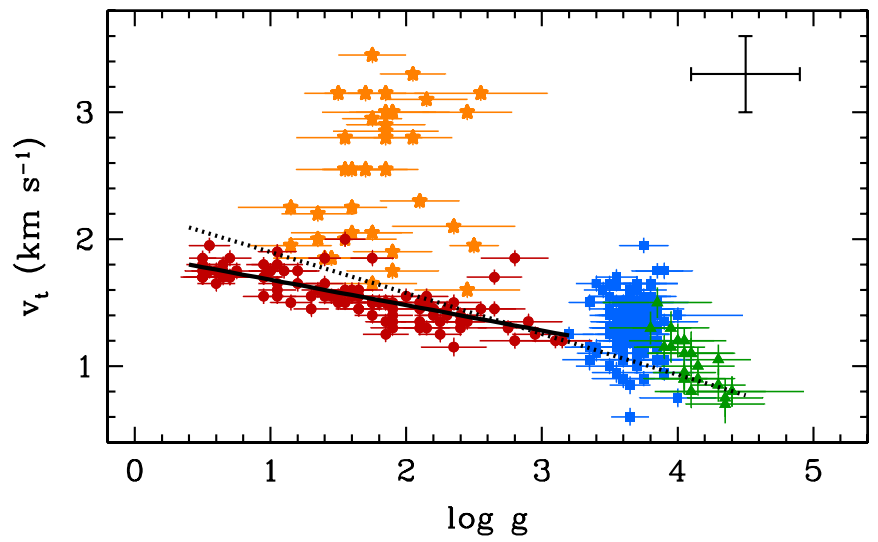

Figure 11. Relationship between $\log g$ and $v_{\mathrm{t}}$. Different evolutionary states are illustrated by different point symbols: red circles mark stars in class RG, blue squares mark stars in class SG, orange starred symbols mark stars in class $\mathrm{HB}$, and green triangles mark stars in class MS. The solid line represents the relationship defined by the RG stars in our sample. The dotted line represents the relationship found by Gratton et al. (1996). Statistical uncertainties are shown on the points, and the black cross in the upper right corner illustrates a typical systematic uncertainty.

(A color version of this figure is available in the online journal.)

the two relations into better agreement overall. No significant relationship appears between $\log g$ and $v_{\mathrm{t}}$ for the stars in our SG, MS, or HB classes.

The standard deviations reported in Table 9 are considerably larger than the statistical uncertainties reported in Table 7, and the difference is likely due to systematic effects. By definition, these are not included in the statistical error budget. Zeropoint differences in $[\mathrm{Fe} / \mathrm{H}]$ are generally insignificant considering the magnitude of the dispersions. We address the source of the remaining zeropoint differences in Section 8.4, and systematic uncertainties are discussed in Section 8.5.

\subsection{The Impact of EW Measurements, Line Lists, Model Grids, and Analysis Codes}

We assess the impact that the EW measurements, line list, $\log g f$ values, and general machinery (i.e., grid of model atmospheres and line analysis software) have on the derived metallicities when compared with previous work. To do this, we rederive iron abundances for stars in common with other studies using published EW measurements, line lists, and model atmosphere parameters. Five recent sets of studies have several stars in common with us: Johnson (2002), the "OZ" project (Carretta et al. 2002; Cohen et al. 2006, 2008), the First Stars project (including Cayrel et al. 2004, Spite et al. 2005, François et al. 2007, and others), Honda et al. (2004a, 2004b), and Lai et al. (2008).

The results of several comparisons are listed in Table 10. In comparison "A," we rederive the iron abundances using published EW measurements, line lists, and model atmosphere parameters from another study, but we use the ATLAS9 grid of model atmospheres and MOOG to perform the calculations. We may think of comparison " $\mathrm{A}$ " as representing the differences in $[\mathrm{Fe} / \mathrm{H}]$ we would have derived if we had used the EW measurements, line lists, and temperature/gravity scales of other studies. In comparison "B," we rederive the iron abundances using the published EW measurements and line lists from other studies, our grid of MARCS models, and MOOG. We may think of comparison "B" as representing the differences in $[\mathrm{Fe} / \mathrm{H}]$ we would have derived if we had adopted other EW measurements and line lists for our analysis. In comparison "C," we rederive the iron abundances using our EW measurements and line list, the model atmosphere parameters from other studies, the ATLAS9 model grid, and MOOG. We may think of comparison " $C$ " as representing the differences in $[\mathrm{Fe} / \mathrm{H}]$ that would have been derived by other studies using our EW measurements and line list.

The final column in Table 10 ("C"-" $\mathrm{C}$ ") lists the differences in derived iron abundances when using stellar parameters from other studies if only the EW measurements and line list are 
Table 10

Comparison of Derived Iron Abundances Using Different Equivalent Widths, Line Lists, and Analysis Tools

\begin{tabular}{|c|c|c|c|c|c|}
\hline $\begin{array}{l}\text { Abundance } \\
\text { Ratio }\end{array}$ & $\begin{array}{c}\text { No. Stars } \\
\text { in Common }\end{array}$ & $\begin{array}{c}\Delta(\sigma) \\
\text { "A" }\end{array}$ & $\begin{array}{c}\Delta(\sigma) \\
\text { "B" }\end{array}$ & $\begin{array}{l}\Delta(\sigma) \\
" C "\end{array}$ & $\begin{array}{c}\Delta(\sigma) \\
\text { "C"-"A" }\end{array}$ \\
\hline \multicolumn{6}{|c|}{ Johnson (2002) } \\
\hline$[\mathrm{Fe} \mathrm{I} / \mathrm{H}]$ & 7 & $-0.114(0.021)$ & $-0.054(0.039)$ & $-0.067(0.032)$ & $+0.047(0.031)$ \\
\hline$[\mathrm{Fe} \mathrm{II} / \mathrm{H}]$ & 7 & $-0.064(0.028)$ & $-0.063(0.032)$ & $-0.010(0.034)$ & $+0.054(0.034)$ \\
\hline \multicolumn{6}{|c|}{ OZ: Carretta et al. (2002); Cohen et al. (2006, 2008) } \\
\hline$[\mathrm{Fe} \mathrm{I} / \mathrm{H}]$ & 5 & $-0.154(0.055)$ & $+0.066(0.046)$ & $-0.214(0.051)$ & $-0.060(0.070)$ \\
\hline$[\mathrm{Fe} \mathrm{II} / \mathrm{H}]$ & 5 & $-0.066(0.055)$ & $-0.142(0.207)$ & $+0.042(0.156)$ & $+0.108(0.172)$ \\
\hline \multicolumn{6}{|c|}{ First stars: Cayrel et al. (2004) } \\
\hline$[\mathrm{Fe} \mathrm{I} / \mathrm{H}]$ & 18 & $-0.027(0.008)$ & $+0.016(0.039)$ & $-0.032(0.046)$ & $-0.005(0.046)$ \\
\hline$[\mathrm{Fe} \mathrm{II} / \mathrm{H}]$ & 18 & $-0.004(0.033)$ & $-0.058(0.068)$ & $+0.039(0.064)$ & $+0.043(0.017)$ \\
\hline \multicolumn{6}{|c|}{ Honda et al. (2004a, 2004b) } \\
\hline$[\mathrm{Fe} \mathrm{I} / \mathrm{H}]$ & 10 & $-0.260(0.021)$ & $-0.037(0.078)$ & $-0.189(0.090)$ & $+0.071(0.082)$ \\
\hline$[\mathrm{Fe}$ II $/ \mathrm{H}]$ & $\ldots$ & $\ldots$ & $\ldots$ & $\ldots$ & $\ldots$ \\
\hline \multicolumn{6}{|c|}{ Lai et al. (2008) } \\
\hline$[\mathrm{Fe} \mathrm{I} / \mathrm{H}]$ & 12 & $-0.007(0.016)$ & $+0.022(0.025)$ & $-0.029(0.025)$ & $-0.023(0.023)$ \\
\hline$[\mathrm{Fe} \mathrm{II} / \mathrm{H}]$ & 12 & $-0.008(0.017)$ & $-0.139(0.075)$ & $+0.125(0.091)$ & $+0.133(0.083)$ \\
\hline
\end{tabular}

Notes. Comparison "A" derives the iron abundances using published EWs and model parameters from the other study and our machinery. Comparison "B" derives the iron abundances using published EWs from the other study, our derived model parameters, and our machinery. Comparison "C" derives the iron abundances using EWs measured by us, stellar parameters derived by the other study, and our machinery. Comparison "C"- "A" is the difference in iron abundances when only the EWs and line lists change. Differences are in the sense of "as derived here" minus "published." Quantities in parenthesis refer to the standard deviation.

changed. We calculate that the $\log g f$ values for $\mathrm{Fe}$ I and Fe II lines in common are different by $<0.03$ dex on average. Thus any residual differences in the final column in Table 10 are the result of which lines are actually used in the analysis.

These comparisons are imperfect. For example, some of these other studies used Turbospectrum, SPTOOL, or employed earlier versions of MOOG that did not include Rayleigh scattering in the source function. When appropriate, we have reverted to the earlier version of MOOG when making these calculations for comparison. The OSMARCS and earlier ATLAS grids of model atmospheres were used in some other studies, and the interpolation codes for the ATLAS9 grid are also different. The adopted solar iron abundance varies from $\log \epsilon(\mathrm{Fe})=7.50$ to 7.52 among these studies, and the scaled solar compositions adopted vary from one model grid to another. Finally, we simply adopt published model atmosphere parameters without rederiving them from scratch before determining the iron abundances.

Nevertheless, Table 10 indicates that the differences in the iron abundances are often small or not significant. It is beyond the scope of the present study to identify the source of the remaining discrepancies. Using the comparison with the First Stars sample as an example, we conclude based on comparison "A" that our analysis would have derived Fe I abundances lower by 0.027 dex using their methods. The differences in the EW measurements and lines used for analysis are negligible, as demonstrated by comparisons "B," "C," "C"- "A," and Figure 4. These comparisons should give some guidance to investigators attempting to place our study in the context of others.

\subsection{Systematic Uncertainties}

The true magnitude of systematic uncertainties is more difficult to quantify. The scatter observed when comparing our model parameters to those in previous studies of stars in common may give some guidance here. For stars in the evolutionary classes $\mathrm{RG} / \mathrm{SG} / \mathrm{HB} / \mathrm{MS}$, when comparing with studies where model parameters were derived by similar techniques, these comparisons yield standard deviations of $151 / 211 / 156 / 71 \mathrm{~K}$ in $T_{\text {eff }}, 0.40 / 0.34 / 0.42 / 0.16$ in $\log g, 0.41 / 0.33 / 0.26 / 0.30 \mathrm{~km} \mathrm{~s}^{-1}$ in $v_{\mathrm{t}}$, and $0.24 / 0.22 / 0.16 / 0.07 \mathrm{dex}$ in $[\mathrm{Fe} \mathrm{II} / \mathrm{H}]$. In most cases these uncertainties dominate the statistical uncertainties.

We may further investigate the scale of systematic uncertainties by comparing our model parameters with those derived by alternative techniques. We compare our spectroscopically derived temperatures with ones derived from color $-T_{\text {eff }}$ relations (hereafter "photometric" temperatures). We compute photometric temperatures from the $J-H$ and $J-K$ relations of Alonso et al. (1999b). This is illustrated in Figure 12, where we show the difference in photometric and spectroscopic temperature for 219 stars in our sample. There is a large amount of scatter at any given temperature, but there is a significant offset (approaching $200 \mathrm{~K}$ on average) for stars cooler than $\sim 5100 \mathrm{~K}$ and $(V-K)_{0}>1.8$. This finding echoes that of many previous studies, including Johnson (2002), Cayrel et al. (2004), Aoki et al. (2007), Cohen et al. (2008), and Lai et al. (2008). The underlying physical cause of this discrepancy is not fully understood, but it likely stems at least in part from the inability of 1D hydrostatic LTE model atmospheres to capture the essential physics of convection, radiative transfer, and so on. This naturally affects the predicted colors and line formation. Frebel et al. (2013) discuss this issue at length and propose an empirical calibration based on seven well-studied nearby stars to bring the two scales into agreement. The relationship between the $T_{\text {eff }}$ differences and $T_{\text {eff }}$ shown in their Figure 2 agrees nearly perfectly with the mean offsets shown in our Figure 10. Frebel et al. also note that the discrepancy between the two scales becomes even more exaggerated for stars with $[\mathrm{Fe} / \mathrm{H}]<-3.5$. We see a similar outcome: for the 27 stars (counting repeat analyses) with $[\mathrm{Fe} / \mathrm{H}]<-3.5$ in common between our sample and previous ones (Figure 10), our $T_{\text {eff }}$ values are lower by $323 \mathrm{~K}(\sigma=90 \mathrm{~K})$ 

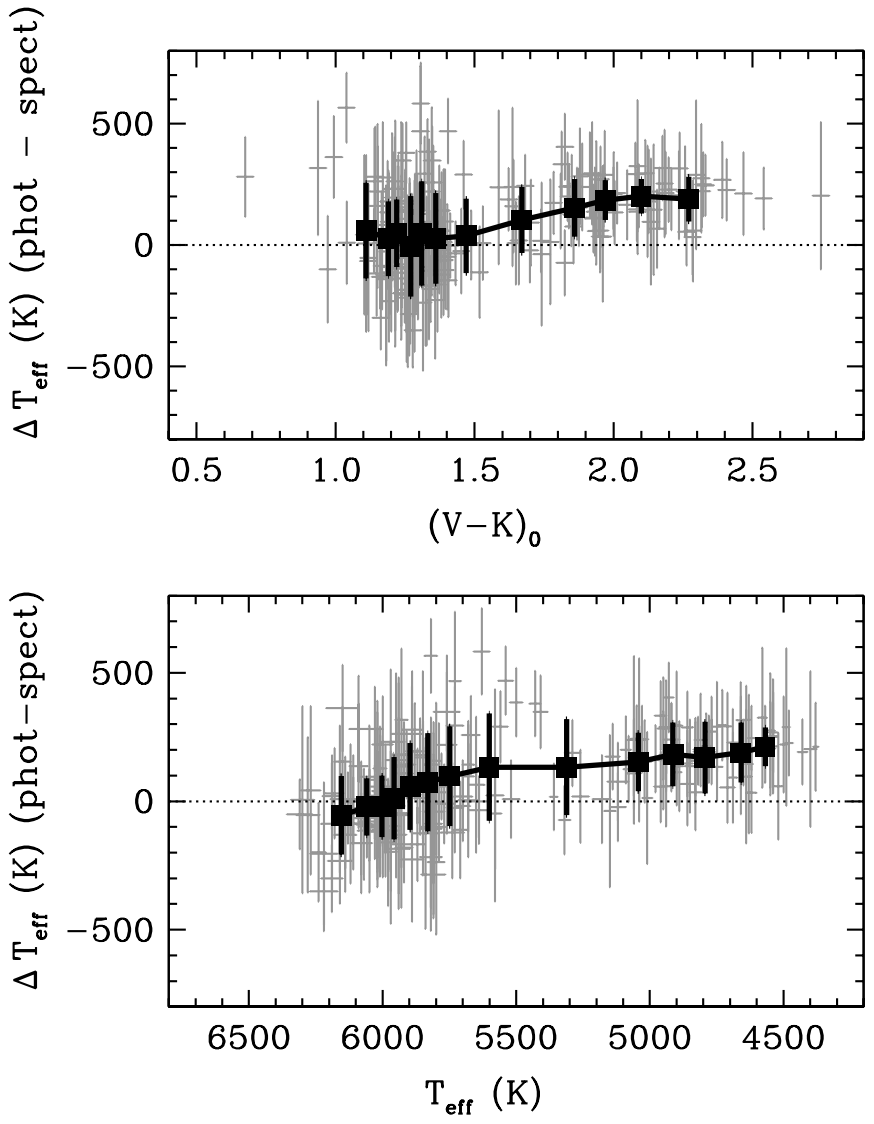

Figure 12. Difference in photometric and spectroscopic $T_{\mathrm{eff}}$ as a function of $(V-K)_{0}$ (top) and spectroscopic $T_{\text {eff }}$ (bottom). The photometric $T_{\text {eff }}$ values are predicted from the Alonso et al. (1999b) $(J-H)$ and $(J-K)$ relations, using the methods described in Section 6. Stars with discrepant photometry are ignored. Only stars evolved beyond the MSTO but not yet on the HB are shown. Gray crosses indicate individual stars, and the large black points mark the weighted average and standard deviation computed by passing a box of 30 stars with an overlap of 15 stars through the data. The dotted line indicates a difference of zero.

on average. This demonstrates that the cooler temperature scale in our study relative to previous studies that used color $-T_{\text {eff }}$ relations is a consequence of the method used to derive $T_{\text {eff }}$, as discussed previously in Section 8.3.

For a few nearby stars, we compare our gravities with those calculated from parallaxes measured by the Hipparcos mission (Perryman 1997), using the data from the reduction by van Leeuwen (2007). We restrict this comparison to stars with Hipparcos parallax precisions better than $20 \%$ and $E(B-$ $V)<0.12$; only 16 stars in our sample meet these criteria. Using the parallax, apparent magnitude, reddening, bolometric corrections (BC; Alonso et al. 1999a), temperature, metallicity, mass (assumed to be $0.8 M_{\odot}$ ), and the solar constants $M_{\mathrm{bol}, \odot}=$ $4.74, T_{\text {eff } \odot}=5780 \mathrm{~K}$, and $\log g_{\odot}=4.44$, we can calculate $\log g$ by means of the relation

$$
\begin{aligned}
\log g= & 0.4\left(M_{K, \star}+\mathrm{BC}_{K}-M_{\mathrm{bol}, \odot}\right)+\log g_{\odot} \\
& +4 \log \left(T_{\mathrm{eff}, \star} / T_{\mathrm{eff}, \odot}\right)+\log \left(m_{\star} / m_{\odot}\right) .
\end{aligned}
$$

Figure 13 compares these "physical" $\log g$ values with our derived $\log g$ values for these 16 stars. We also compare our gravity for HD 122563 with that derived from the recent measurement of its angular diameter by Creevey et al. (2012). Our $\log g$ values are systematically lower than the physical ones by $0.49 \operatorname{dex}(\sigma=0.32 \mathrm{dex})$, independent of the method we have

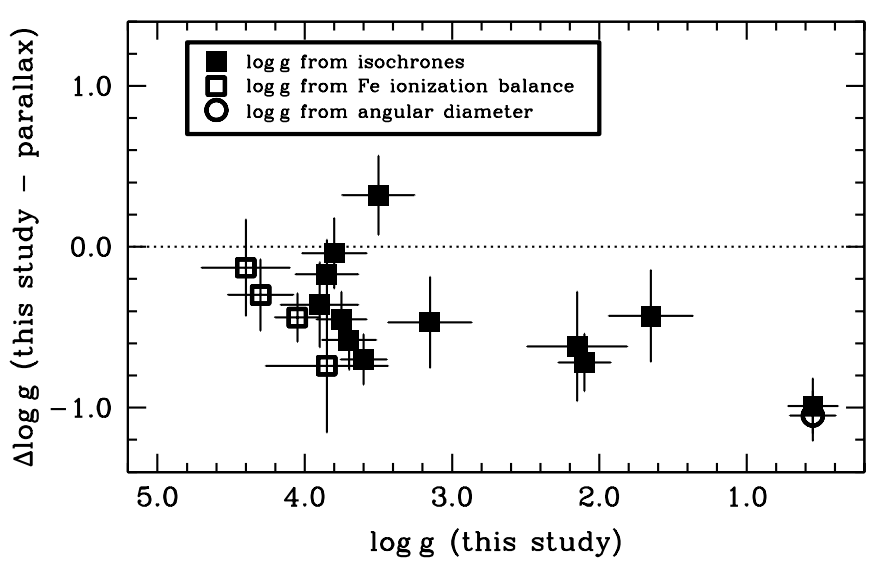

Figure 13. Comparison of $\log g$ derived in this study with those computed from Hipparcos parallaxes. Only the 16 stars with $E(B-V)<0.12$ and Hipparcos uncertainties smaller than $20 \%$ are considered. The fill and shape of the points indicates the method by which the $\log g$ was obtained. For display purposes, the difference in the "angular diameter" measurement is plotted alongside the "parallax" measurements, but these are really two separate methods to derive $\log g$. The dotted line indicates a difference of zero.

used to derive $\log g$. This offset is similar to that found $(-0.57$, $\sigma=0.42$ ) when comparing our $\log g$ values with those calculated from color $-T_{\text {eff }}$ relations by other investigators (Section 8.3).

We use the $T_{\text {eff }}$ predicted by $(V-K)_{0}$ instead of a spectroscopically derived $T_{\text {eff }}$ as an alternative method to calculate $\log g$ from isochrones. For the 11 stars to which we can apply both the parallax method and the isochrone method, the photometric temperatures give $\log g$ values greater by only 0.15 dex than the physical ones. This would be encouraging if not for the fact that the dispersion in $\log g$ for the photometric $T_{\text {eff }}$ input is substantially larger $(\sigma=0.63 \mathrm{dex})$ than that using the spectroscopic $T_{\text {eff }}$ input $(\sigma=0.36 \mathrm{dex})$.

Figure 14 shows the difference in the derived iron abundances using Fe II or Fe I lines. Differences are mostly found in the range $-0.1 \mathrm{dex}<\left[\mathrm{Fe}\right.$ II $\left./ \mathrm{Fe}_{\mathrm{I}}\right]<+0.3 \mathrm{dex}$, with a mean difference of 0.10 dex $(\sigma=0.13$ dex $)$. These differences do not show a strong dependence on metallicity, but they are slightly larger for the warmest $(\approx 6100 \mathrm{~K})$ and coolest $(\approx 4600 \mathrm{~K})$ stars in the sample. The differences are comparable to what is expected if overionization of $\mathrm{Fe}_{\mathrm{I}}$ is responsible (e.g., Thévenin \& Idiart 1999).

We suggest that the statistical uncertainties, listed in Table 7, should be considered when comparing abundance ratios of stars with similar parameters. The total uncertainties, which are more difficult to assess, should be used when absolute abundances are considered. The values listed at the beginning of this section may be considered representative of the total uncertainties.

\subsection{The Impact of $\log g$ on Metallicity}

Our $\log g$ values are systematically lower than those implied by several other derivation methods. As demonstrated in Section 9.1, ionized species are more sensitive to $\log g$ than neutral species are. Our metallicities are based on the iron abundance derived from Fe II lines, so this difference does have an impact.

Tables 9 and 10 can be used to quantify this impact on the iron abundances. Two of the sets of comparisons listed, the $0 \mathrm{Z}$ studies and the Lai et al. (2008) study, calculated photometric $T_{\text {eff }}$ values and used these to calculate $\log g$ from isochrones. Their values of $T_{\text {eff }}$ and $\log g$ are equivalent to what we would have calculated 

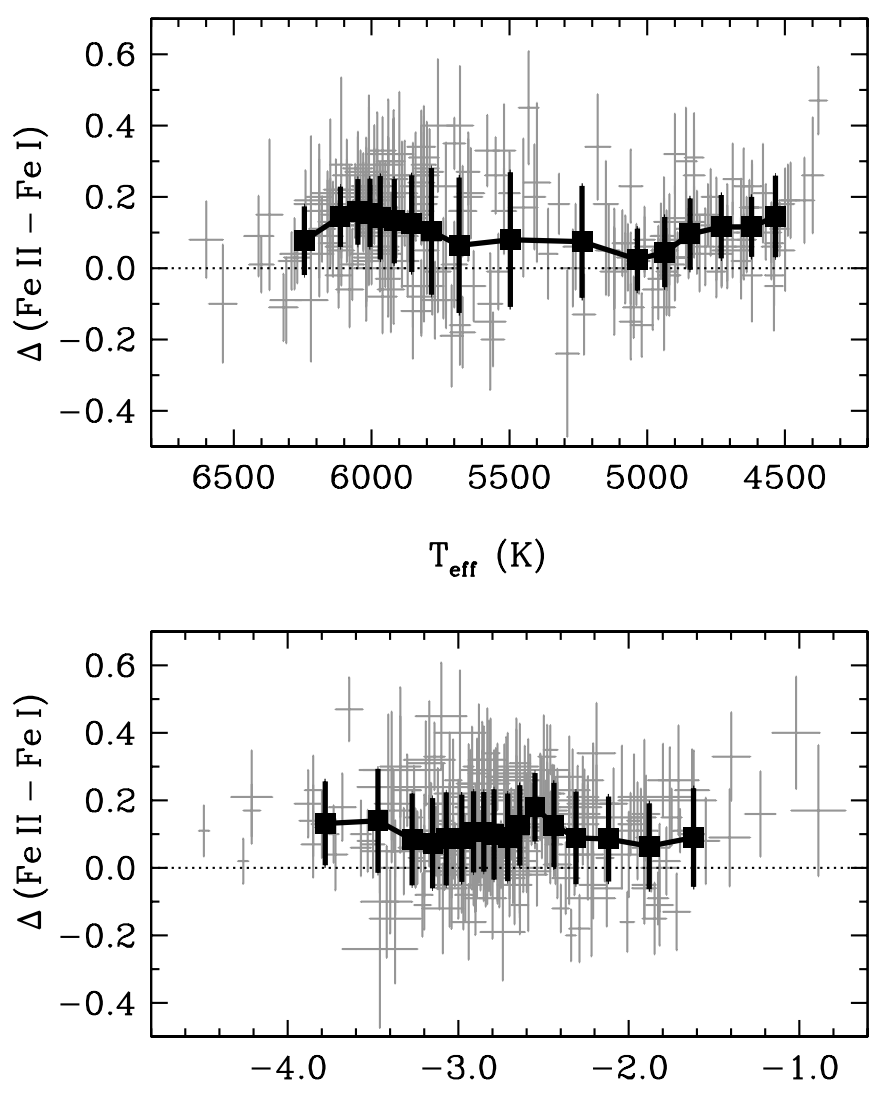

$[\mathrm{Fe} \mathrm{II} / \mathrm{H}]$

Figure 14. Difference in iron abundance derived from $\mathrm{Fe}$ II and $\mathrm{Fe}$ I as a function of $T_{\text {eff }}$ and [Fe II/H]. Only stars in the SG and RG classes are shown. Gray crosses indicate individual stars, and the large black points mark the weighted average and standard deviation computed by passing a box of 30 stars with an overlap of 15 stars through the data. The dotted line indicates a difference of zero.

using this approach. Note that the $0 \mathrm{Z}$ set of studies listed in Table 10 did not have enough stars in common to merit inclusion in Table 9, so we perform an identical comparison separately and list the results here. For the five stars in common, the mean differences are $-227 \mathrm{~K}(\sigma=169 \mathrm{~K})$ in $T_{\text {eff }},-0.48 \mathrm{dex}(\sigma=$ $0.42 \mathrm{dex})$ in $\log g,-0.55 \mathrm{~km} \mathrm{~s}^{-1}\left(\sigma=0.22 \mathrm{~km} \mathrm{~s}^{-1}\right)$ in $v_{\mathrm{t}}$, and $-0.15 \mathrm{dex}(\sigma=0.22 \mathrm{dex})$ in $[\mathrm{Fe} \mathrm{II} / \mathrm{H}]$.

These results suggest that our metallicities are lower by 0.15 dex (0Z) or 0.08 dex (Lai et al. 2008) in a straight-up comparison. Comparison " $\mathrm{C}$ " in Table 10 lists the $[\mathrm{Fe} \mathrm{I} / \mathrm{H}]$ and $[\mathrm{Fe} \mathrm{II} / \mathrm{H}]$ abundances that we would have derived for our sample using others' photometric $T_{\text {eff }}$ and $\log g$ values with our EW measurements, line list, model grid, and MOOG. These corrections must be applied to the values above. Thus, we conclude that our metallicities are lower by 0.19 dex $(0 Z)$ or 0.20 dex (Lai et al.) because we have used spectroscopic $T_{\text {eff }}$ values to predict $\log g$ using the $\mathrm{Y}^{2}$ isochrones.

For the stars in common with the $0 \mathrm{Z}$ and Lai et al. (2008) studies, using our approach the mean $[\mathrm{Fe} I \mathrm{II} / \mathrm{Fe} \mathrm{I}]$ ratio is $+0.16 \pm 0.04 \operatorname{dex}(\sigma=0.15 \mathrm{dex})$. Using the alternate approach the mean is $+0.12 \pm 0.02 \mathrm{dex}(\sigma=0.08 \mathrm{dex})$. We also divide these stars into classes of giants or subgiants. For the giants, using the alternate approach would reduce the mean difference from $+0.20 \pm 0.04(\sigma=0.14)$ to $+0.14 \pm 0.02(\sigma=0.07)$. For the subgiants, the differences are even smaller, $+0.10 \pm 0.07$ $(\sigma=0.17)$ using our approach and $+0.09 \pm 0.04(\sigma=0.10)$ using the alternate approach. These results suggest that our ap- proach may slightly overestimate the amount of overionization occurring for Fe I in the giants.

\subsection{Iron Abundance Trends with Wavelength}

Roederer et al. (2012) identified a relationship between wavelength and iron abundance derived from Fe I lines in four metalpoor giants, and Lawler et al. (2013) found a similar effect in a metal-poor turnoff star. This effect is characterized as a decrease in the average abundance by $\approx 0.05$ to 0.20 dex at short wavelengths (mainly $2280<\lambda<4000 \AA$ ) compared to long wavelengths $(\lambda>4400 \AA)$. Roederer et al. investigated several explanations for this effect and favored an unidentified extra source of continuous opacity at short wavelengths that was not accounted for. Ultimately that study only adopted an empirical correction to the abundances derived from lines at short wavelengths so as to match the abundances derived from longer wavelengths. The four stars in that study are also in our study (HD 108317, HD 122563, HD 126238, and HD 128279). Our derived temperatures are different by 30 to $70 \mathrm{~K}$ because of the different techniques used to derive the model atmosphere parameters. We do not find a similar effect for these four stars, although the number of Fe I lines studied at short wavelengths is considerably smaller than the sample examined by Roederer et al.

We take advantage of our large sample of stars to improve the statistics at short wavelengths. Figure 15 illustrates the wavelength dependence of abundances derived from $\mathrm{Fe}$ I lines in our study for 68 giants and 142 subgiants. Abundances derived from $\mathrm{Fe}$ I lines at short wavelengths are, on average, lower by 0.04 dex or less than lines at long wavelengths. The results do not change when comparing smaller subsets of stars with similar $T_{\text {eff }}$ and metallicity. This is a very small difference compared to what Roederer et al. (2012) found, and the difference may be related to the methods used to derive model parameters. Roederer et al. derived model parameters mainly using lines at long wavelengths, only considering the abundances from lines at short wavelengths once the model parameters had been set. In this study, we have included all Fe I lines when deriving the model parameters. This choice was necessary because a substantial part of our sample is warmer and more metal-poor than the stars considered by Roederer et al., hence fewer lines at long wavelengths are available to us. If lines at short wavelength yield systematically lower abundances, they will preferentially be culled by our $2 \sigma$-clipping algorithm described in Section 8.1. Our experience suggests that this is a possibility, so the results shown in Figure 15 should be taken as lower limits to the differences at short wavelengths.

Lines of other species are not affected by the $2 \sigma$-clipping algorithm but would be affected if there is a physical origin of this effect, like missing continuous opacity. We devote our attention in Section 9.3 to finding other evidence of such an effect.

\subsection{Other Considerations}

McWilliam et al. (1995a) noted that the first (uppermost) levels of the grids of model atmospheres available at the time, those of Bell et al. (1976) and Kurucz, ${ }^{5}$ began at different atmosphere layers. For a typical metal-poor giant (BD-18 5550), these models began at column masses ("RHOX") near 13 and $0.2 \mathrm{~g} \mathrm{~cm}^{-2}$, respectively. In the Bell et al. model, the optical

\footnotetext{
5 Cited by McWilliam et al. (1995a) as R. L. Kurucz 1992, private communication.
} 

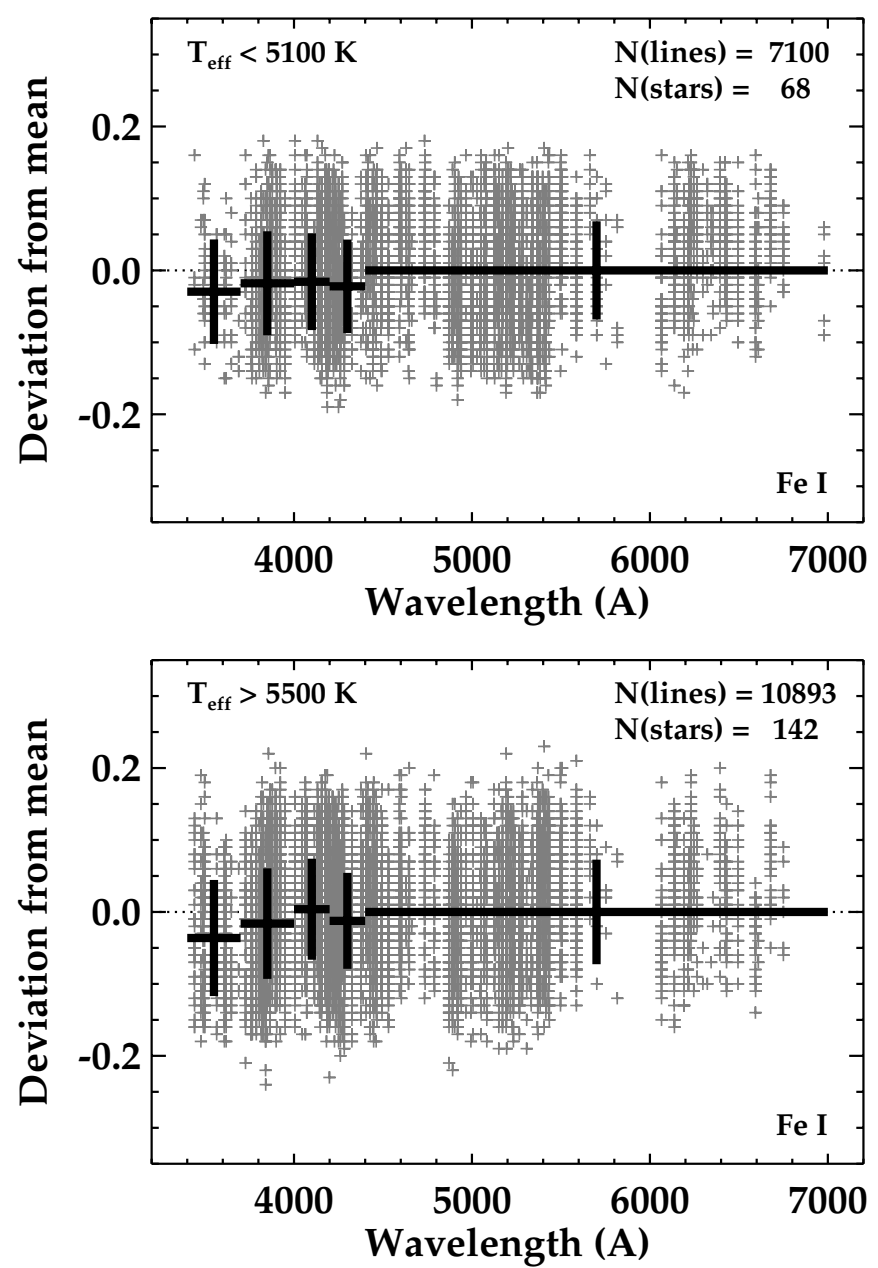

Figure 15. Line-by-line abundances derived from Fe I lines as a function of wavelength. The top panel shows lines in stars on the upper giant branch with $T_{\text {eff }}<5100 \mathrm{~K}$, and the bottom panel shows lines in stars with $T_{\text {eff }}>5500 \mathrm{~K}$ and cooler than the MSTO. Small gray crosses mark individual lines, and bold black crosses mark the mean and standard deviation in each of several wavelength bins. All abundances are normalized to the mean abundance for each star derived from lines with $\lambda>4400 \AA$, which is marked by the dotted black line.

depth at line center of lines stronger than $\log (\mathrm{EW} / \lambda)>-4.7$ was nonzero in the first layer, indicating that a significant portion of the line was formed above this layer. While the Kurucz models covered the entire line-forming region, they too failed to include the temperature inversion expected in the low density layers of the chromosphere. This introduced a different set of problems since the cores of strong lines may be formed in these layers. Models of BD-18 5550 from the current MARCS and ATLAS9 grids extend to 1.3 and $0.02 \mathrm{~g} \mathrm{~cm}^{-2}$, respectively. This covers the line-forming region for lines up to $\log (\mathrm{EW} / \lambda) \gg-4.0$ in the ATLAS9 models and $\log (\mathrm{EW} / \lambda)=-4.45$ in the MARCS models. Fortuitously, $\log (\mathrm{EW} / \lambda)=-4.45$ is also the typical upper bound for the EWs we have measured in giants, so both model grids treat the lines in our data set comparably in this regard. Tests by McWilliam et al. (1995a) to account for the presence of a chromosphere indicate a zero-point uncertainty of approximately 0.1 dex for Fe I lines with $\log (\mathrm{EW} / \lambda)>-4.7$ in metal-poor giants. This issue is not resolved by the updated models and will affect our results for the giants. For typical metal-poor dwarf or subgiant models we obtain similar results, in that the MARCS models do not encompass the entire line-forming region for $\log (\mathrm{EW} / \lambda)>-4.50$.
Fe I lines with $\log (\mathrm{EW} / \lambda)>-4.50(-4.45)$ comprise less than $2.0 \%(0.7 \%)$ of the total Fe I lines in our sample and are unlikely to skew the derived atmospheric parameters significantly. This could, however, impact species whose abundance can only be deduced from strong lines. In Section 9.3 we consider systematic abundance offsets associated with different lines of the same species.

Using spherically symmetric model atmospheres with an analysis code that treats the line-forming layers as parallel slabs can lead to systematic errors in the derived abundances. The spherical models have a slightly lower temperature structure in the uppermost layers due to dilution of the radiation field from lower layers (Gustafsson et al. 2008). We find that the magnitude of this effect is small, less than a few percent in the most extreme cases (i.e., stars with very low surface gravity), where the main difference lies in optically thin layers with $\log \tau<-1$. We therefore make no corrections for this effect.

The current version of MOOG includes Rayleigh scattering from neutral $\mathrm{H}$ atoms in the source function (Sobeck et al. 2011). Our calculations indicate that opacity from Rayleigh scattering contributes $14 \%$ of the total continuous opacity at $3700 \AA$ in typical metal-poor red giants at $\tau=1$. To the extent that the Rayleigh scattering contribution is being properly computed, it should be included. (The other dominant contribution to the continuous opacity in these stars comes from boundfree absorption by $\mathrm{H}^{-}$.) In typical metal-poor subgiants, the contribution from Rayleigh scattering is much less than $1 \%$ at all wavelengths considered.

The classical technique of deriving the microturbulence velocity from Fe I lines, which we have used, may systematically overestimate $v_{\mathrm{t}}$ because of correlated errors in the measured EWs and derived abundances (Magain 1984). Theoretical EWs computed from the stellar model could eliminate this particular bias. Mucciarelli (2011) has reexamined the situation, finding that the two methods produce equivalent results when moderately high $\mathrm{S} / \mathrm{N}$ data are used $(\mathrm{S} / \mathrm{N} \gtrsim 70 / 1)$. Our data generally fall in this regime since we are not typically measuring weak lines (i.e., those most prone to the bias) at blue wavelengths where the $\mathrm{S} / \mathrm{N}$ is lower. Even in our HET spectra, where the $\mathrm{S} / \mathrm{N}$ is lowest, no more than $4 \%$ of our Fe I lines would be susceptible to this bias. We do not pursue the matter further.

Distances calculated from absolute magnitudes computed from spectroscopically derived $T_{\text {eff }}$ or $\log g$ will be systematically overestimated relative to distances calculated from photometric determinations of $T_{\text {eff }}$ or $\log g$. Any analysis that makes use of these distances to examine the kinematic properties of our sample will be affected. A proper analysis of the stellar kinematics is deferred for future work.

\section{ABUNDANCE ANALYSIS}

On average, we derive abundances or upper limits from 216 $(\sigma=48)$ of the 474 lines considered in each star. For all lines with an EW measurement listed in Table 5, we use MOOG to compute theoretical EWs that are forced to match the measured EWs by adjusting the input abundance. For lines denoted "synth" in Table 5, we use MOOG to generate a series of synthetic spectra where the abundance of the element producing the line of interest is varied to match the observed spectrum. Each synthesis spans $\pm 3 \AA$ on either side of this line. We generate the line lists used in the synthesis using the Kurucz $\&$ Bell (1995) line lists as a starting point. We then replace the $\log g f$ values of the line of interest and any other lines with data from laboratory or more detailed theoretical studies, where 
Table 11

Abundances Derived from Individual Lines

\begin{tabular}{lccrc}
\hline \hline Star & Species & $\begin{array}{c}\text { Wavelength } \\
(\AA)\end{array}$ & $\log \epsilon$ & $\sigma$ \\
\hline CS 22166-016 & Li I & 6707.80 & $<0.44$ & $\ldots$ \\
CS 22166-016 & Mg I & 4057.51 & 5.02 & 0.17 \\
CS 22166-016 & Mg I & 4167.27 & 5.07 & 0.18 \\
CS 22166-016 & Mg I & 5172.68 & 5.15 & 0.39 \\
CS 22166-016 & Mg I & 5183.60 & 5.02 & 0.37 \\
CS 22166-016 & Mg I & 5528.40 & 5.13 & 0.19 \\
\hline
\end{tabular}

(This table is available in its entirety in machine-readable and Virtual Observatory (VO) forms in the online journal. A portion is shown here for guidance regarding its form and content.)

available. Occasionally, especially for lines in the blue regions of the spectrum, we are forced to alter the theoretical $\log g f$ values to produce a reasonable fit to the observed spectrum. These lists are then employed, unchanged, for syntheses of all stars in the sample. We do allow for abundance variations of elements other than the one of interest based on our EW analysis for each star. Abundances derived from lines of $\mathrm{Li}$ I, CH, CN, NH, Al I, Sc II, V I, V II, Mn I, Mn II, Co I, CuI, and elements with $Z>30$ are determined via the synthesis method. All others are derived using EWs.

We estimate an upper limit on the abundance for lines not detected in our spectra. The $3 \sigma$ upper limits are calculated from a version of the formula presented on p. 590 of Frebel et al. (2008b), which itself is derived from Equation (A8) of Bohlin et al. (1983). When multiple lines of the same species are not detected, we adopt the upper limit that provides the strongest constraint on the abundance.

The presence of multiple isotopes of some lines of interest may lead to small energy shifts in the transition wavelength, and our syntheses account for the IS for Ba II, Nd II, Sm II, Eu II, $\mathrm{Yb}$ II, Ir I, and $\mathrm{Pb}$ I. In all cases we adopt the $r$-process isotopic ratios presented in Sneden et al. (2008) unless our analysis reveals a substantial contribution of $s$-process material. In that case, we adopt an appropriate mix of $r$ - and $s$-process isotopes based on our derived abundances.

Abundances and uncertainties for each line in each star are reported in Table 11. The mean abundances and uncertainties are presented in Table 12. The full versions of Tables 11 and 12 are available in the online edition of the journal. The meanings of the different uncertainty estimates in Table 12 are discussed in Section 9.1. The ratios relative to the solar values, $[\mathrm{X} / \mathrm{H}]$ or $[\mathrm{X} / \mathrm{Fe}]$, where $\mathrm{X}$ stands for any metal, are computed relative to the Asplund et al. (2009) solar photospheric abundances, listed in Table 13. In cases where the photospheric value is poorly known, we adopt the abundance measured in CI-type carbonaceous meteorites instead. These cases are noted in Table 13. We remind readers that $[\mathrm{X} / \mathrm{Fe}]$ ratios are constructed using the abundances derived from species in the same ionization state; i.e., neutrals to neutrals and ions to ions. Only $\log \epsilon$ abundances or upper limits, not $[\mathrm{X} / \mathrm{Fe}]$ ratios, are presented for lithium and technetium.

Molecular abundances are derived by spectrum synthesis. For the NH lines near $3360 \AA$, we adopt the Kurucz \& Bell (1995) line list with $\log g f$ values reduced by a factor of two and a dissociation potential of $3.45 \mathrm{eV}$, as recommended by Johnson et al. (2007). For the CN lines near $3880 \AA$, we adopt the Kurucz $\&$ Bell line lists without change and a dissociation potential of $7.65 \mathrm{eV}$. For the $\mathrm{CH}$ lines near $4310 \AA$, we adopt the line list of B. Plez (2007, private communication) and a dissociation potential of $3.47 \mathrm{eV}$. We adopt a default fitting uncertainty of $0.15 \mathrm{dex}$ for the $\mathrm{CH}$ lines, $0.20 \mathrm{dex}$ for the $\mathrm{CN}$ lines, and 0.30 dex for the $\mathrm{NH}$ lines. The final abundances listed in Table 12 reflect the $\mathrm{CH}$ and $\mathrm{NH}$ abundances, when possible, otherwise the nitrogen abundance is derived from the $\mathrm{CN}$ abundance after carbon has been set using the $\mathrm{CH}$ lines. Molecular formation in cool stellar atmospheres is sensitive to the temperature and density, especially the presence and degree of granulation found in $3 \mathrm{D}$ hydrodynamical models (e.g., Collet et al. 2007). These effects are difficult to quantify. Our 1D LTE results should be treated with due caution when using them for anything besides gross discriminants of carbon and nitrogen enrichment.

For all lines with $\lambda>5670 \AA$, we examine the stellar spectrum simultaneously with a telluric spectrum of Earth's atmosphere (Hinkle et al. 2000). In general, we did not observe hot telluric standards during our observing program, so any lines that appear to be compromised by telluric absorption are discarded from further consideration. Furthermore, the Na I D lines are sometimes blended with interstellar sodium absorption. We do not attempt to derive abundances from these lines when they appear asymmetric, broadened beyond the typical stellar line widths, or when the telluric spectrum suggests that they may be compromised.

Several stars in our sample have low $[\alpha / \mathrm{Fe}]$ ratios, so using the $\alpha$-enhanced grid of models may not, in principle, be appropriate. To test how much of an effect this may have on our analysis, we have performed the abundance analysis for several key species using an $\alpha$-enhanced model and an $\alpha$ normal model for the two most $\alpha$-poor stars in our sample, G004-036 and BD + 80 245. The results of this test are listed in Table 14. For G004-036, a subgiant, the differences in derived $\log \epsilon$ are all smaller than $0.007 \mathrm{dex}$, and ratios constructed among abundances derived from like ionization states differ by 0.002 dex or less. For BD +80245 , the differences in derived $\log \epsilon$ are larger than 0.002 dex only for ionized species, for which the differences are as large as 0.019 dex. Ratios constructed among abundances derived from like ionization states differ by 0.005 dex or less. Differences among abundances derived from unlike ionization states, e.g., [Fe I/Fe II], are $0.021 \mathrm{dex}$ or smaller, and we (again) advise against constructing abundance ratios this way. For stars with intermediate $[\alpha / \mathrm{Fe}]$ ratios, these differences will be smaller. On the basis of this test, we conclude that using the $\alpha$-enhanced models for all stars in the sample will have, at most, a minimal effect on the derived abundance ratios.

\subsection{Uncertainties}

We estimate abundance uncertainties following the formalism presented in McWilliam et al. (1995a). The standard deviation of a single line is calculated according to Equation (A5) of McWilliam et al. To evaluate the partial derivatives, we have selected model atmospheres representing stars in our sample on the red giant branch, subgiant branch, HB, and MS. Then, following McWilliam et al., we alter the model parameters one by one to estimate the change in the abundance that results in fictitious iron lines whose strength spans the full range of line strengths considered in our sample. We repeat this exercise for both neutral lines and singly ionized lines.

The results of this exercise are illustrated in Figures 16 through 18. As expected, lines of neutral atoms are more sensitive to $T_{\text {eff }}$ than lines of ionized atoms are, and the opposite is true for pressure sensitivity $(\log g)$. For strong lines, the microturbulence velocity parameter dominates the 
Table 12

Mean Abundances

\begin{tabular}{|c|c|c|c|c|c|c|c|c|}
\hline Star & Species & $N_{\text {lines }}$ & $\log \epsilon$ & {$[\mathrm{X} / \mathrm{Fe}]^{\mathrm{a}}$} & $\sigma_{\text {statistical }}$ & $\sigma_{\text {total }}$ & $\sigma_{\text {neutrals }}$ & $\sigma_{\text {ions }}$ \\
\hline CS $22166-016$ & $\mathrm{Fe} I$ & 96 & 4.28 & -3.22 & 0.06 & 0.15 & 0.00 & 0.00 \\
\hline CS 22166-016 & Fe II & 11 & 4.41 & -3.09 & 0.07 & 0.15 & 0.00 & 0.00 \\
\hline CS 22166-016 & Li I & 1 & $<0.52$ & $\ldots$ & $\ldots$ & $\ldots$ & $\ldots$ & $\ldots$ \\
\hline CS $22166-016$ & $\mathrm{C}(\mathrm{CH})$ & 1 & 5.35 & 0.01 & 0.15 & 0.25 & 0.19 & 0.19 \\
\hline CS 22166-016 & $\mathrm{N}(\mathrm{NH})$ & 1 & 5.34 & 0.60 & 0.30 & 0.36 & 0.32 & 0.32 \\
\hline CS 22166-016 & OI & 0 & $\ldots$ & $\ldots$ & $\ldots$ & $\ldots$ & $\ldots$ & $\ldots$ \\
\hline CS 22166-016 & $\mathrm{NaI}$ & 0 & $\ldots$ & $\ldots$ & $\ldots$ & $\ldots$ & $\ldots$ & $\ldots$ \\
\hline CS 22166-016 & $\operatorname{Mg}_{I}$ & 5 & 5.07 & 0.69 & 0.05 & 0.15 & 0.08 & 0.18 \\
\hline CS 22166-016 & $\mathrm{Al}$ I & 1 & 2.56 & -0.67 & 0.09 & 0.29 & 0.24 & 0.30 \\
\hline CS 22166-016 & Si I & 0 & $\ldots$ & $\ldots$ & $\ldots$ & $\ldots$ & $\ldots$ & $\ldots$ \\
\hline CS 22166-016 & $\mathrm{K}_{\mathrm{I}}$ & 0 & $\ldots$ & $\ldots$ & $\ldots$ & $\ldots$ & $\ldots$ & $\ldots$ \\
\hline CS 22166-016 & $\mathrm{Ca} \mathrm{I}$ & 11 & 3.73 & 0.62 & 0.11 & 0.17 & 0.12 & 0.20 \\
\hline CS 22166-016 & Sc II & 6 & 0.01 & -0.05 & 0.06 & 0.15 & 0.17 & 0.10 \\
\hline CS 22166-016 & Ti I & 15 & 2.01 & 0.29 & 0.07 & 0.15 & 0.09 & 0.18 \\
\hline CS 22166-016 & Ti II & 22 & 2.08 & 0.22 & 0.06 & 0.15 & 0.17 & 0.09 \\
\hline CS 22166-016 & $\mathrm{V}_{\mathrm{I}}$ & 1 & 0.68 & -0.03 & 0.14 & 0.19 & 0.15 & 0.22 \\
\hline CS 22166-016 & V II & 2 & 0.99 & 0.15 & 0.20 & 0.24 & 0.26 & 0.21 \\
\hline CS 22166-016 & CrI & 6 & 2.27 & -0.15 & 0.06 & 0.14 & 0.08 & 0.18 \\
\hline CS 22166-016 & Cr II & 2 & 2.63 & 0.08 & 0.11 & 0.18 & 0.20 & 0.13 \\
\hline CS 22166-016 & Mn I & 4 & 1.78 & -0.42 & 0.08 & 0.16 & 0.10 & 0.20 \\
\hline CS 22166-016 & Mn II & 4 & 1.74 & -0.60 & 0.15 & 0.21 & 0.23 & 0.17 \\
\hline CS 22166-016 & CoI & 5 & 1.77 & 0.00 & 0.14 & 0.20 & 0.15 & 0.23 \\
\hline CS 22166-016 & Ni I & 6 & 3.07 & 0.07 & 0.11 & 0.21 & 0.16 & 0.23 \\
\hline CS 22166-016 & $\mathrm{Cu} \mathrm{I}$ & 0 & $\ldots$ & $\ldots$ & $\ldots$ & $\ldots$ & $\ldots$ & $\ldots$ \\
\hline CS 22166-016 & $\mathrm{Zn} \mathrm{I}$ & 0 & $\ldots$ & $\ldots$ & $\ldots$ & $\ldots$ & $\ldots$ & $\ldots$ \\
\hline CS 22166-016 & Ga I & 0 & $\ldots$ & $\ldots$ & $\ldots$ & $\ldots$ & $\ldots$ & $\ldots$ \\
\hline CS 22166-016 & $\mathrm{Rb} \mathrm{I}_{\mathrm{I}}$ & 0 & $\ldots$ & $\ldots$ & $\ldots$ & $\ldots$ & $\ldots$ & $\ldots$ \\
\hline CS 22166-016 & Sr II & 2 & -0.50 & -0.28 & 0.04 & 0.25 & 0.25 & 0.25 \\
\hline CS 22166-016 & Y II & 1 & -1.41 & -0.53 & 0.10 & 0.17 & 0.20 & 0.12 \\
\hline CS 22166-016 & $\mathrm{Zr}$ II & 3 & -0.57 & -0.06 & 0.12 & 0.18 & 0.21 & 0.14 \\
\hline CS 22166-016 & $\mathrm{Nb}$ II & 1 & $<0.12$ & 1.75 & $\ldots$ & $\ldots$ & $\ldots$ & $\ldots$ \\
\hline CS 22166-016 & Mo I & 1 & $<-0.41$ & 0.93 & $\ldots$ & $\ldots$ & $\ldots$ & $\ldots$ \\
\hline CS 22166-016 & $\mathrm{Tc} \mathrm{I}$ & 1 & $<-0.11$ & $\ldots$ & $\ldots$ & $\ldots$ & $\ldots$ & $\ldots$ \\
\hline CS 22166-016 & Ru I & 0 & $\ldots$ & $\ldots$ & $\ldots$ & $\ldots$ & $\ldots$ & $\ldots$ \\
\hline CS 22166-016 & Sn I & 1 & $<0.95$ & 2.10 & $\ldots$ & $\ldots$ & $\ldots$ & $\ldots$ \\
\hline CS 22166-016 & Ba II & 3 & -1.35 & -0.44 & 0.05 & 0.15 & 0.17 & 0.09 \\
\hline CS 22166-016 & La II & 1 & -2.07 & -0.08 & 0.18 & 0.22 & 0.24 & 0.19 \\
\hline CS 22166-016 & $\mathrm{Ce}$ II & 5 & $<-1.62$ & -0.11 & $\ldots$ & $\ldots$ & $\ldots$ & $\ldots$ \\
\hline CS 22166-016 & Pr II & 1 & -1.84 & 0.53 & 0.17 & 0.22 & 0.24 & 0.18 \\
\hline CS 22166-016 & Nd II & 1 & -1.85 & -0.18 & 0.11 & 0.18 & 0.20 & 0.13 \\
\hline CS 22166-016 & Sm II & 0 & $\ldots$ & $\ldots$ & $\ldots$ & $\ldots$ & $\ldots$ & $\ldots$ \\
\hline CS 22166-016 & Eu II & 3 & -2.37 & 0.19 & 0.13 & 0.19 & 0.21 & 0.15 \\
\hline CS 22166-016 & Gd II & 2 & $<-1.21$ & 0.81 & $\ldots$ & $\ldots$ & $\ldots$ & $\ldots$ \\
\hline CS 22166-016 & Tb II & 0 & $\ldots$ & $\ldots$ & $\ldots$ & $\ldots$ & $\ldots$ & $\ldots$ \\
\hline CS 22166-016 & Dy II & 1 & -1.70 & 0.29 & 0.14 & 0.20 & 0.22 & 0.15 \\
\hline CS 22166-016 & Ho II & 0 & $\ldots$ & $\ldots$ & $\ldots$ & $\ldots$ & $\ldots$ & $\ldots$ \\
\hline CS 22166-016 & Er II & 1 & -1.70 & 0.47 & 0.40 & 0.43 & 0.44 & 0.41 \\
\hline CS 22166-016 & Tm II & 0 & $\ldots$ & $\ldots$ & $\ldots$ & $\ldots$ & $\ldots$ & $\ldots$ \\
\hline CS 22166-016 & Yb II & 1 & -2.10 & 0.07 & 0.12 & 0.19 & 0.21 & 0.14 \\
\hline CS 22166-016 & Hf II & 2 & $<-0.94$ & 1.30 & $\ldots$ & $\ldots$ & $\ldots$ & $\ldots$ \\
\hline CS 22166-016 & Ir I & 0 & $\ldots$ & $\ldots$ & $\ldots$ & $\ldots$ & $\ldots$ & $\ldots$ \\
\hline CS 22166-016 & $\mathrm{Pb}$ & 1 & $<0.53$ & 1.71 & $\ldots$ & $\ldots$ & $\ldots$ & $\ldots$ \\
\hline CS 22166-016 & Th II & 3 & $<-1.95$ & 1.08 & $\ldots$ & $\ldots$ & $\ldots$ & $\ldots$ \\
\hline
\end{tabular}

Notes. ${ }^{\mathrm{a}}[\mathrm{Fe} / \mathrm{H}]$ is indicated for Fe I and Fe II.

(This table is available in its entirety in machine-readable and Virtual Observatory (VO) forms in the online journal. A portion is shown here for guidance regarding its form and content.)

uncertainties. In practice, we fit the relationships shown in Figures 16 through 18 by polynomial functions (often just constants over much of the range of line strength) and use these in our calculations. The uncertainty in abundance resulting from uncertainty in the EW measurement is estimated in a similar manner by altering the line strength by $1 \mathrm{~m} \AA$, as shown in
Figure 19. As expected, this corresponds to a proportionally larger uncertainty for weaker lines. In practice, we adopt a wavelength-dependent uncertainty for the EW based on the median $\mathrm{S} / \mathrm{N}$ ratios.

The cross term for $T_{\text {eff }}$ and $\log g$ in Equation (A5) of McWilliam et al. (1995a) is evaluated using the procedure 
Table 13

Adopted Solar Abundances

\begin{tabular}{|c|c|c|}
\hline Element & $Z$ & $\log \epsilon$ \\
\hline $\mathrm{Li}$ & 3 & $\ldots$ \\
\hline $\mathrm{C}$ & 6 & 8.43 \\
\hline $\mathrm{N}$ & 7 & 7.83 \\
\hline $\mathrm{O}$ & 8 & 8.69 \\
\hline $\mathrm{Na}$ & 11 & 6.24 \\
\hline $\mathrm{Mg}$ & 12 & 7.60 \\
\hline $\mathrm{Al}$ & 13 & 6.45 \\
\hline $\mathrm{Si}$ & 14 & 7.51 \\
\hline K & 19 & 5.03 \\
\hline $\mathrm{Ca}$ & 20 & 6.34 \\
\hline $\mathrm{Sc}$ & 21 & 3.15 \\
\hline $\mathrm{Ti}$ & 22 & 4.95 \\
\hline V & 23 & 3.93 \\
\hline $\mathrm{Cr}$ & 24 & 5.64 \\
\hline Mn & 25 & 5.43 \\
\hline $\mathrm{Fe}$ & 26 & 7.50 \\
\hline Co & 27 & 4.99 \\
\hline $\mathrm{Ni}$ & 28 & 6.22 \\
\hline $\mathrm{Cu}$ & 29 & 4.19 \\
\hline $\mathrm{Zn}$ & 30 & 4.56 \\
\hline $\mathrm{Ga}$ & 31 & 3.04 \\
\hline $\mathrm{Rb}$ & 37 & 2.52 \\
\hline $\mathrm{Sr}$ & 38 & 2.87 \\
\hline $\mathrm{Y}$ & 39 & 2.21 \\
\hline $\mathrm{Zr}$ & 40 & 2.58 \\
\hline $\mathrm{Nb}$ & 41 & 1.46 \\
\hline Mo & 42 & 1.88 \\
\hline $\mathrm{Tc}$ & 43 & $\ldots$ \\
\hline $\mathrm{Ru}$ & 44 & 1.75 \\
\hline $\mathrm{Sn}$ & 50 & $2.07^{\mathrm{a}}$ \\
\hline $\mathrm{Ba}$ & 56 & 2.18 \\
\hline $\mathrm{La}$ & 57 & 1.10 \\
\hline $\mathrm{Ce}$ & 58 & 1.58 \\
\hline $\operatorname{Pr}$ & 59 & 0.72 \\
\hline $\mathrm{Nd}$ & 60 & 1.42 \\
\hline $\mathrm{Sm}$ & 62 & 0.96 \\
\hline $\mathrm{Eu}$ & 63 & 0.52 \\
\hline $\mathrm{Gd}$ & 64 & 1.07 \\
\hline $\mathrm{Tb}$ & 65 & 0.30 \\
\hline Dy & 66 & 1.10 \\
\hline Ho & 67 & 0.48 \\
\hline $\mathrm{Er}$ & 68 & 0.92 \\
\hline $\mathrm{Tm}$ & 69 & 0.10 \\
\hline $\mathrm{Yb}$ & 70 & $0.92^{\mathrm{a}}$ \\
\hline Hf & 72 & 0.85 \\
\hline Ir & 77 & 1.38 \\
\hline $\mathrm{Pb}$ & 82 & $2.04^{\mathrm{a}}$ \\
\hline $\mathrm{Th}$ & 90 & $0.06^{\mathrm{a}}$ \\
\hline
\end{tabular}

Note. ${ }^{a}$ Meteoritic abundance.

outlined by Johnson (2002). For each of our model atmospheres of representative stars, we compare the $\log g$ parameters derived when altering the input $T_{\text {eff }}$ by an amount corresponding to a random draw of $T_{\text {eff }}$ from a normal distribution with $\sigma=\sigma_{T_{\text {eff }}}$, where $\sigma_{T_{\text {eff }}}$ is typical for stars in each evolutionary state. We repeat this exercise 10 times for each covariance for a representative case for each evolutionary state. The covariance, $\sigma_{T \log g}$, is then estimated according to Equation (3) of Johnson (2002). For stars in the RG, SG, HB, and MS classes, $\sigma_{T} \log g$ is $5.1,1.2,2.8$, and $2.2 ; \sigma_{\log g v t}$ is $-0.04,-0.02,-0.03$, and -0.01 ; and $\sigma_{v t T}$ is $44,38,45$, and 16 . The $1 \sigma$ uncertainties are listed for each line in each star in Table 11.

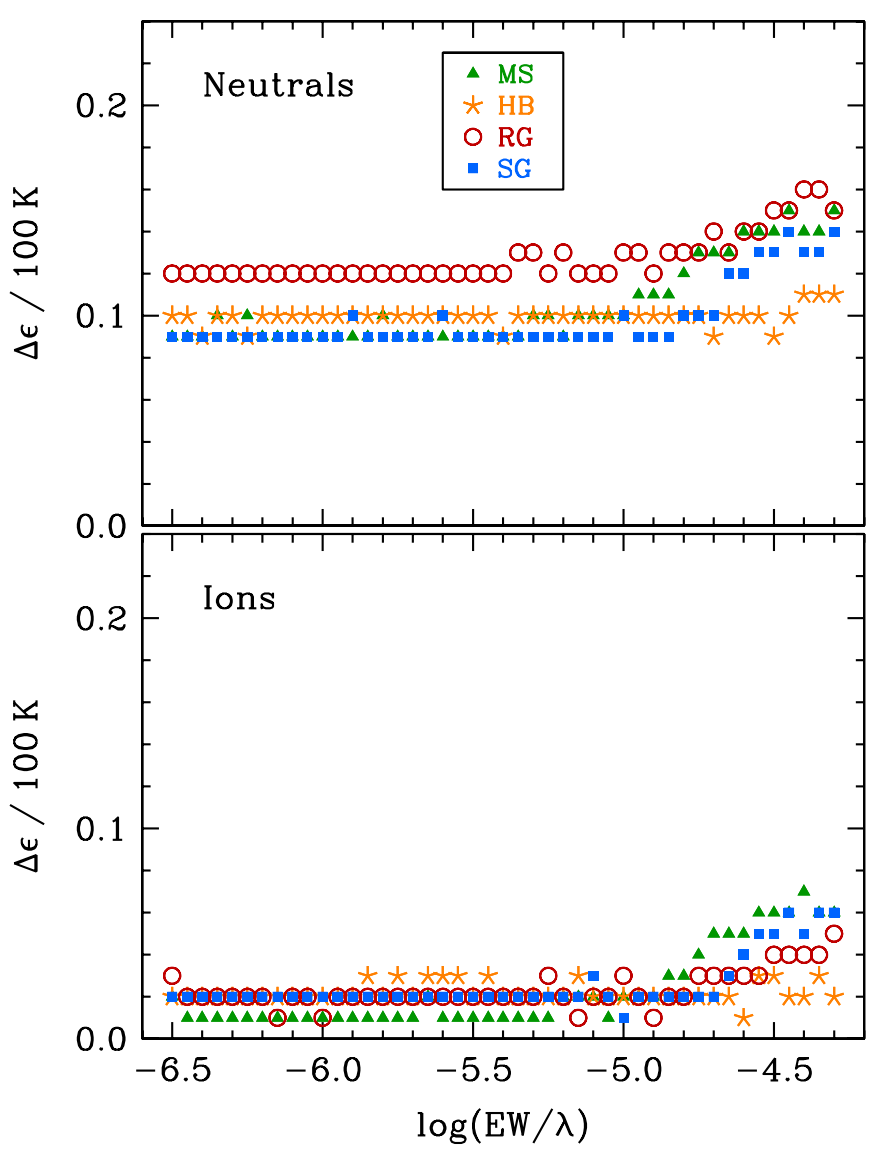

Figure 16. Change in derived abundances of neutral lines (top) and singly ionized lines (bottom) resulting from a change in $T_{\text {eff }}$ of $100 \mathrm{~K}$.

(A color version of this figure is available in the online journal.)

Table 14

Abundance Differences Derived from $\alpha$-enhanced and $\alpha$-normal Models

\begin{tabular}{|c|c|c|c|c|c|c|}
\hline \multirow[t]{2}{*}{ Species or Ratio } & \multicolumn{3}{|c|}{ G004-036 } & \multicolumn{3}{|c|}{$\mathrm{BD}+80245$} \\
\hline & $\Delta$ & $\sigma$ & $N_{\text {lines }}$ & $\Delta$ & $\sigma$ & $N_{\text {lines }}$ \\
\hline $\log \epsilon\left(\mathrm{Mg}_{\mathrm{I}}\right)$ & +0.004 & 0.005 & 7 & +0.010 & 0.012 & 4 \\
\hline $\log \epsilon(\mathrm{Ca} I)$ & +0.004 & 0.005 & 10 & +0.001 & 0.005 & 11 \\
\hline $\log \epsilon(\mathrm{Ti} \mathrm{I})$ & +0.005 & 0.005 & 15 & -0.002 & 0.004 & 13 \\
\hline $\log \epsilon$ (Ti II $)$ & +0.007 & 0.005 & 19 & +0.019 & 0.003 & 17 \\
\hline $\log \epsilon(\mathrm{Cr} I)$ & +0.003 & 0.005 & 9 & +0.000 & 0.004 & 11 \\
\hline $\log \epsilon(\mathrm{Cr}$ II $)$ & +0.007 & 0.006 & 3 & +0.013 & 0.006 & 3 \\
\hline $\log \epsilon\left(\mathrm{Fe}_{\mathrm{I}}\right)$ & +0.003 & 0.005 & 87 & +0.002 & 0.006 & 66 \\
\hline $\log \epsilon(\mathrm{Fe}$ II $)$ & +0.006 & 0.005 & 10 & +0.017 & 0.005 & 10 \\
\hline $\log \epsilon(\mathrm{Ni} \mathrm{I})$ & +0.006 & 0.005 & 7 & +0.001 & 0.004 & 8 \\
\hline$\left[\mathrm{Mg} \mathrm{I} / \mathrm{Fe}_{\mathrm{I}}\right]$ & +0.001 & 0.007 & $\ldots$ & +0.008 & 0.013 & $\ldots$ \\
\hline$[\mathrm{Ca} \mathrm{I} / \mathrm{Fe} \mathrm{I}]$ & +0.001 & 0.007 & $\ldots$ & -0.002 & 0.008 & $\ldots$ \\
\hline [Ti I/Fe I] & +0.001 & 0.007 & $\ldots$ & -0.005 & 0.007 & $\ldots$ \\
\hline [Ti II/Fe II] & +0.001 & 0.007 & $\ldots$ & +0.002 & 0.006 & $\ldots$ \\
\hline$[\mathrm{Cr} \mathrm{I} / \mathrm{Fe} \mathrm{I}]$ & +0.000 & 0.007 & $\ldots$ & -0.002 & 0.007 & $\ldots$ \\
\hline [Cr II/Fe II $]$ & +0.001 & 0.008 & $\ldots$ & -0.004 & 0.008 & $\ldots$ \\
\hline [Ni I/Fe I] & +0.002 & 0.007 & $\ldots$ & -0.001 & 0.007 & $\ldots$ \\
\hline [Ti I/Ti II] & -0.003 & 0.007 & $\ldots$ & -0.021 & 0.006 & $\ldots$ \\
\hline [Cr I/Cr II] & -0.003 & 0.008 & $\ldots$ & -0.013 & 0.007 & $\ldots$ \\
\hline$[\mathrm{Fe}$ I/Fe II $]$ & -0.003 & 0.007 & $\ldots$ & -0.015 & 0.008 & $\ldots$ \\
\hline
\end{tabular}

We compute mean abundances weighted by the uncertainty given by Equation (A5) of McWilliam et al. (1995a). These abundances are reported in Table 12. Several sets of uncertainties are listed in this table. The statistical uncertainty, $\sigma_{\text {statistical }}$, is that given by Equation (A17) of McWilliam et al., which 


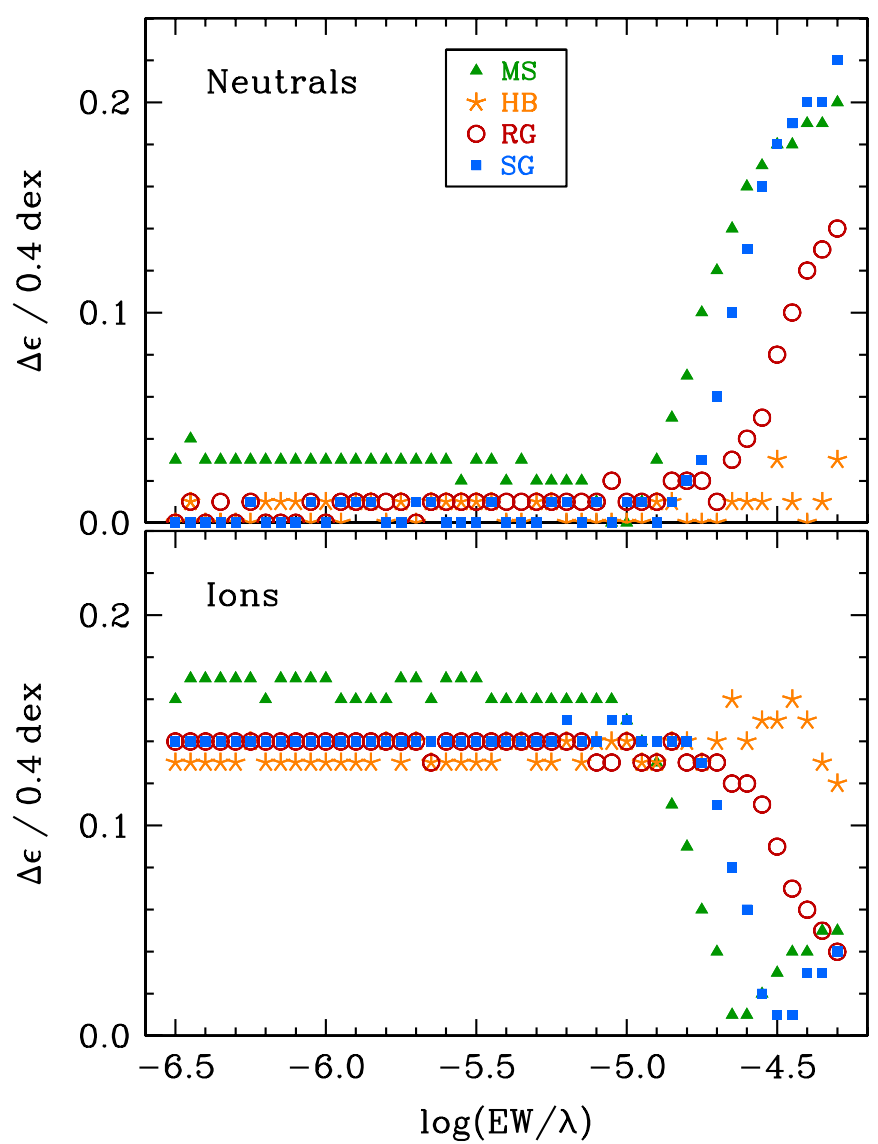

Figure 17. Change in derived abundances of neutral lines (top) and singly ionized lines (bottom) resulting from a change in $\log g$ of 0.4 dex. Symbols are the same as in Figure 16.

(A color version of this figure is available in the online journal.)

includes uncertainties in the EW measurement and $\log g f$ values. This uncertainty generally decreases as the number of lines examined increases, although we have forced an artificial minimum uncertainty of $0.02 \mathrm{dex}$ to guard against unreasonably small values. The total uncertainty, $\sigma_{\text {total }}$, is that given by Equation (A16) of McWilliam et al. This includes the statistical uncertainty and uncertainties in the model atmosphere parameters and does not decrease appreciably as the number of lines increases. For this calculation, we adopt the estimates of the systematic uncertainties in the model atmosphere parameters given in Section 8.5. The remaining two uncertainties listed in Table 12 are approximations of the abundance ratio uncertainties given by Equations (A19) and (A20) of McWilliam et al. Rather than calculating and presenting this uncertainty for every possible element pair, we have computed these uncertainties using $\mathrm{Fe}_{\mathrm{I}}$ and Fe II as representative cases. We suggest that $\sigma_{\text {neutrals }}$ for element A should be added in quadrature with $\sigma_{\text {statistical }}$ for element B when computing the ratio [A/B] when $\mathrm{B}$ is derived from neutral lines. Similarly, we suggest that $\sigma_{\text {ions }}$ for element $\mathrm{A}$ should be added in quadrature with $\sigma_{\text {statistical }}$ for element B when element B is derived from ionized lines. (The uncertainty in the ratio $[\mathrm{B} / \mathrm{A}]$ may not necessarily equal that for [A/B], but in general they are comparable.)

\subsection{Corrections for Departures from LTE in the Line Formation Calculations}

Great effort in recent years has been dedicated to identifying transitions that are not well represented by the assumptions of

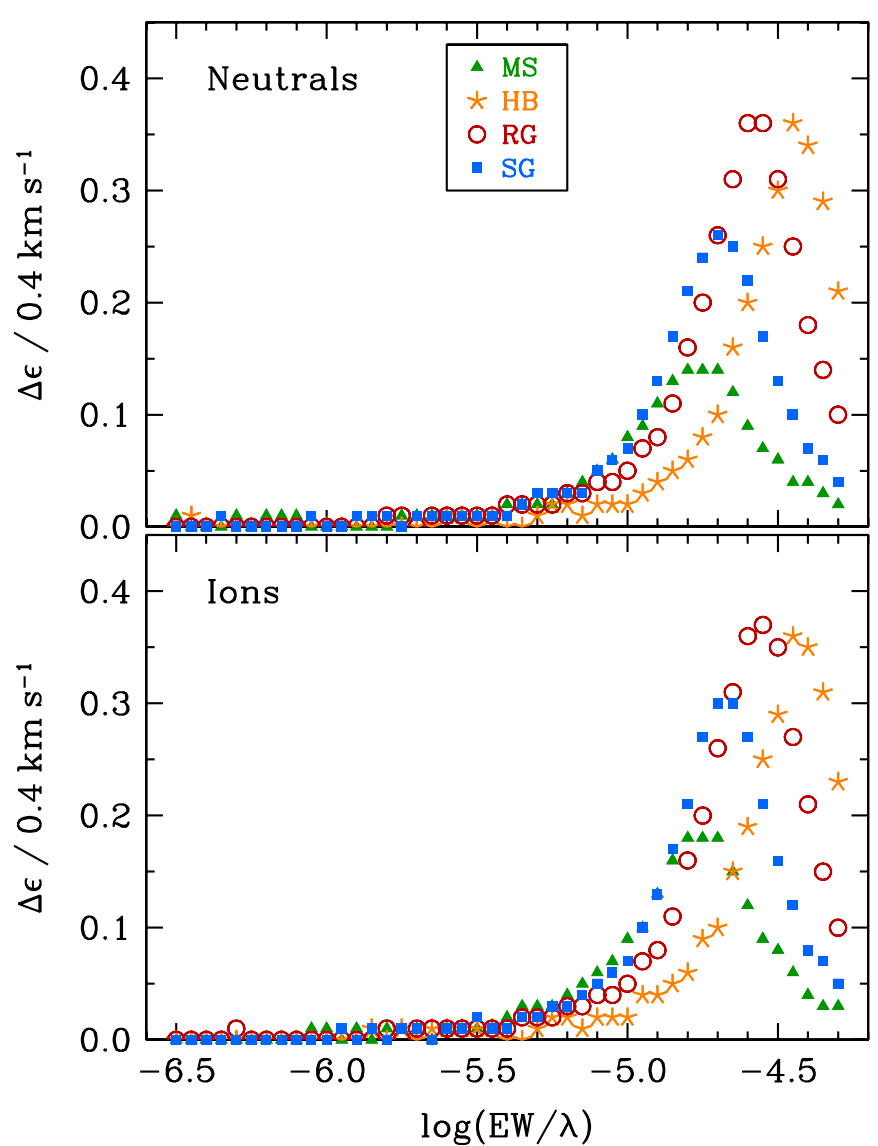

Figure 18. Change in derived abundances of neutral lines (top) and singly ionized lines (bottom) resulting from a change in $v_{\mathrm{t}}$ of $0.4 \mathrm{~km} \mathrm{~s}^{-1}$. Symbols are the same as in Figure 16.

(A color version of this figure is available in the online journal.)

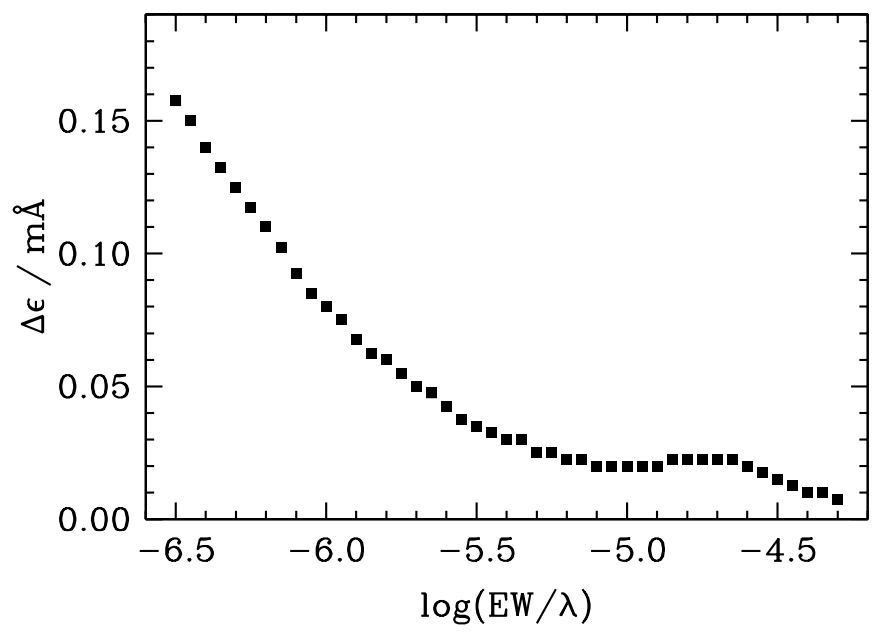

Figure 19. Change in derived abundances of lines of various strength resulting from a change in $\mathrm{EW}$ of $1 \mathrm{m \AA}$.

LTE in late-type stellar atmospheres. We adopt non-LTE corrections for the abundances derived from lines of $\mathrm{Li}$ I, O I, Na I, and $\mathrm{KI}$. Grids of non-LTE calculations spanning a range of stellar parameters and metallicities have been presented for various lines of these species, as discussed below. The abundances presented in Tables 11 and 12 reflect these corrections on Li I, $\mathrm{O}$ I, NaI, and KI. For investigators who wish to make use of our uncorrected LTE abundances of these species, we list the corrections in Table 15. The complete version of this table is 
Table 15

Non-LTE Abundance Corrections

\begin{tabular}{|c|c|c|c|c|c|c|c|c|c|c|}
\hline \multirow[t]{2}{*}{ Star } & \multirow{2}{*}{$\begin{array}{c}\text { Li I } \\
6707\end{array}$} & \multicolumn{3}{|c|}{ O I } & \multicolumn{4}{|c|}{$\mathrm{NaI}$} & \multicolumn{2}{|c|}{$\mathrm{K}_{\mathrm{I}}$} \\
\hline & & 7771 & 7774 & 7775 & 5682 & 5688 & 5889 & 5895 & 7664 & 7698 \\
\hline CS 22166-016 & +0.08 & $\ldots$ & $\ldots$ & $\ldots$ & $\ldots$ & $\ldots$ & $\ldots$ & $\ldots$ & $\ldots$ & $\ldots$ \\
\hline CS 22169-008 & -0.03 & -0.22 & -0.22 & $\ldots$ & $\ldots$ & $\ldots$ & $\ldots$ & $\ldots$ & $\ldots$ & $\ldots$ \\
\hline CS 22169-035 & +0.14 & -0.05 & -0.05 & -0.05 & $\ldots$ & $\ldots$ & $\ldots$ & $\ldots$ & $\ldots$ & -0.27 \\
\hline CS 22171-031 & -0.04 & -0.26 & -0.26 & -0.25 & $\ldots$ & $\ldots$ & $\ldots$ & $\ldots$ & $\ldots$ & $\ldots$ \\
\hline CS 22171-037 & -0.03 & -0.46 & -0.45 & -0.45 & $\ldots$ & $\ldots$ & -0.05 & -0.05 & $\ldots$ & $\ldots$ \\
\hline
\end{tabular}

(This table is available in its entirety in machine-readable and Virtual Observatory (VO) forms in the online journal. A portion is shown here for guidance regarding its form and content.)

available only in the online edition of the journal. Lines of other species may not be formed in LTE, but grids of non-LTE abundance calculations for these species in late-type stars are not readily available.

For the Li I $6707 \AA$ line, we use the corrections computed by Lind et al. (2009). For the few stars with parameters outside the grid of their calculations $\left(4000 \mathrm{~K} \leqslant T_{\text {eff }} \leqslant 8000 \mathrm{~K}\right.$, $1.0 \leqslant \log g \leqslant 5.0,1.0 \mathrm{~km} \mathrm{~s}^{-1} \leqslant v_{\mathrm{t}} \leqslant 5.0 \mathrm{~km} \mathrm{~s}^{-1}$, $-5.0 \leqslant[\mathrm{Fe} / \mathrm{H}] \leqslant 0$ ), we use the value at the nearest point on the grid. The corrections are generally positive for cool stars and negative for warm stars. The corrections for upper limits are calculated based on the $3 \sigma \mathrm{EW}$ estimated from the $\mathrm{S} / \mathrm{N}$ measurements, so they should be treated with caution. Most corrections are small, $< \pm 0.05$ dex, but a few are as large as \pm 0.13 dex for lines detected. We include an additional 0.05 dex statistical uncertainty in our error estimates to account for uncertainties in the corrections.

The O I triplet at 7771,7774 , and $7775 \AA$ is not formed in LTE, and we adopt the corrections presented by Fabbian et al. (2009). For the few stars with parameters outside the grid of their calculations $\left(4500 \mathrm{~K} \leqslant T_{\text {eff }} \leqslant 6500 \mathrm{~K}, 2.0 \leqslant \log g \leqslant\right.$ $5.0,-3.0 \leqslant[\mathrm{Fe} / \mathrm{H}] \leqslant 0$ ), we use the value at the nearest point on the grid. We include an additional 0.1 dex statistical uncertainty in our error estimates to account for uncertainties in the corrections. After correcting these abundances, however, the resulting oxygen abundances are, on average, $0.50 \pm 0.06 \mathrm{dex}$ $(\sigma=0.25$ dex $)$ higher than those derived from the [O I] $6300 \AA$ line in the seven stars where both abundance indicators could be reliably measured. The [O I] $6300 \AA$ line is generally considered to be a reliable abundance indicator formed under conditions of LTE (Kiselman 2001). The offset between abundances derived from $\left[\mathrm{O}_{\mathrm{I}}\right]$ and $\mathrm{O}_{\mathrm{I}}$ is reminiscent of the result found by García Pérez et al. (2006) in metal-poor giants. We apply a correction for this offset as discussed in Section 9.3.

The lines of the Na I D resonance doublet at 5898 and $5895 \AA$ are not formed under conditions of LTE. We adopt corrections to our LTE abundances using the grid presented by Lind et al. (2011). The corrections are always negative, in the sense that LTE underestimates the line strength and overestimates the abundance. Lind et al. also present corrections for the higher excitation Na I 5682 and $5688 \AA$ lines. We also include these corrections, which are generally small $(<0.1 \mathrm{dex})$ for consistency. For the few stars with parameters outside the grid of their calculations $\left(4000 \mathrm{~K} \leqslant T_{\text {eff }} \leqslant 8000 \mathrm{~K}, 1.0 \leqslant \log g \leqslant 5.0\right.$, $\left.1.0 \mathrm{~km} \mathrm{~s}^{-1} \leqslant v_{\mathrm{t}} \leqslant 5.0 \mathrm{~km} \mathrm{~s}^{-1},-5.0 \leqslant[\mathrm{Fe} / \mathrm{H}] \leqslant+0.5\right)$, we use the value at the nearest point on the grid. We include an additional 0.1 dex statistical uncertainty in our error estimates to account for uncertainties in the corrections.
Observational challenges have limited studies of potassium in metal-poor stars. Only two $\mathrm{K}_{\mathrm{I}}$ lines are routinely detectable in late-type metal-poor stars, the 7664 and $7698 \AA$ K I resonance doublet. These stellar lines are often contaminated with atmospheric $\mathrm{O}_{2}$ lines. Our spectra extend redward enough to observe these lines. As in the case of the Na I resonance doublet discussed in Section 9, we only measure EWs of these lines when one or both appears well separated from the model telluric absorption spectrum. These resonance lines are likely formed out of LTE, and we adopt corrections for the $7698 \AA$ line from the grid of Takeda et al. (2002). Corrections for the $7664 \AA$ line are made from an analogous grid kindly sent by Y. Takeda (2007, private communication). The corrections are almost always negative, in the sense that LTE underestimates the line strength and overestimates the abundance. For stars with parameters outside the grid of their calculations $\left(4500 \mathrm{~K} \leqslant T_{\text {eff }} \leqslant 6500 \mathrm{~K}\right.$, $1.0 \leqslant \log g \leqslant 5.0,1.0 \mathrm{~km} \mathrm{~s}^{-1} \leqslant v_{\mathrm{t}} \leqslant 3.0 \mathrm{~km} \mathrm{~s}^{-1}$, $-3.0 \leqslant[\mathrm{Fe} / \mathrm{H}] \leqslant 0$ ), we use the value at the nearest point on the grid. We report detections of the K I 7664 and $7698 \AA$ lines in 41 stars and 72 stars, respectively, for a total of 98 stars with potassium abundance derivations. Both lines are detected in 15 stars, and the corrected abundances agree well in these stars: $\Delta=-0.019 \pm 0.024(\sigma=0.093)$. We include an additional 0.1 dex statistical uncertainty in our error estimates to account for uncertainties in the corrections.

\subsection{Line-by-line Abundance Offsets and Corrections}

One challenge in producing a homogeneous abundance data set for stars spanning several dex in metallicity is that the set of useful lines for analysis changes from metal-poor to metalrich stars. If systematic line-to-line differences in the derived abundances persist, they will masquerade as subtle changes in the abundance trends. While observers are generally aware of this effect (e.g., Cohen et al. 2004 discuss it in detail for the case of $\mathrm{Mg}$ I lines ${ }^{6}$ ), limitations in the size of the stellar sample often preclude attempts to characterize it reliably for large numbers of elements. This is of little consolation to those wishing to make use of abundance tables to constrain chemical evolution models. We attempt to account for these effects by leveraging our large data set to identify and correct for lines that are systematically discrepant. In this section we discuss the process we use to identify those lines and the empirical corrections that we apply to the abundances.

\footnotetext{
6 The line-by-line differences for five Mg I lines listed in Table 12 of Cohen et al. (2004) agree in sign but not magnitude with ours in Table 16 after correcting for the different $\log g f$ values and number of lines examined. Their corrections would range from +0.16 dex for the $4057 \mathrm{~A}$ line to $-0.23 \mathrm{dex}$ for the $5183 \AA$ line if computed on the same $\log g f$ scale and in the same manner as ours. We cannot trace the source of this difference.
} 
These differences may originate from inaccurate $\log g f$ values or damping constants, departures from LTE in the line-forming layers, poor estimates of the continuous opacity, misidentification of the continuum, or unidentified blends. We can estimate which effects may dominate. The line density is highest in the blue region of the spectrum. If unidentified blends are the dominant source, the abundances we derive from blue lines should be higher on average than abundances derived from red lines, so the corrections would preferentially skew negative for blue lines. The corrections are both positive and negative in the blue region of the spectrum. This indicates that unidentified blends are not the main source of systematic uncertainty here. The magnitude of the corrections is higher in the blue than in the red, however. This suggests that higher line density and lower $\mathrm{S} / \mathrm{N}$ ratios are rendering the continuum placement more uncertain. Simply adopting different analysis techniques-for example, using spectrum synthesis in place of a traditional EW analysis_-does not offer a panacea. The dominant effects are likely to be uncertain continuum placement in the blue region of the spectrum and the deficiencies of using LTE to model the line formation.

For most species, we determine the corrections using the following process. We separate the stars into groups of different evolutionary classes (the RG, SG, HB, MS, and BS categories presented in Section 8.1). For each star in each group, using only stars with large numbers of lines of a given species measured, we compute the difference between each line's resulting abundance and the mean abundance in that star. Within each evolutionary group, we then compute the mean and standard deviation of these differences. Finally, we correct all abundances from a given line in all stars of a given evolutionary group by subtracting these mean differences. These corrections are listed in Table 16. For example, we find that the Mg I $5183 \AA$ line in the SG class yields abundances higher than the mean by $0.09 \mathrm{dex}(\sigma=0.08$, $N=49$ stars). Therefore we reduce the abundance derived from the $5183 \AA$ line by 0.09 dex for all stars in the SG class. As a penalty incurred for making this statistical correction, we add in quadrature the standard deviation, in this case $0.08 \mathrm{dex}$, with the statistical uncertainty. Typically these uncertainties are not the dominant component of the statistical error budget. We apply all corrections, even if some are not statistically meaningful, to preserve the overall mean abundance of the sample. Some evolutionary groups do not contain enough measurements of a particular line to make a reliable assessment of the mean offset, in which case no correction is made. These cases are denoted by blanks in Table 16. A few species present unique challenges that require minor modifications to this process, as discussed below.

We use four sodium abundance indicators in our analysis, the Na I resonance doublet at 5889 and $5895 \AA$ and the higher excitation doublet at 5682 and $5688 \AA$. After correcting for nonLTE effects, the two lines within each doublet yield abundances in excellent agreement with each other on average: $\Delta_{5889-5895}=$ $-0.02 \pm 0.02(\sigma=0.06)$ and $\Delta_{5688-5682}=-0.01 \pm 0.03(\sigma=$ 0.13 ). There are only two stars where three or four of these lines could be measured reliably, so we are unable to assess whether they differ systematically. Furthermore, there are insufficient data to assess systematic differences among stars of different evolutionary state, so we adopt a single set of corrections for all stars.

Previous studies (e.g., Preston et al. 2006a) have revealed relationships between $[\mathrm{Si} / \mathrm{Fe}]$ and $T_{\text {eff }}$ when the silicon abundance is derived from the Si I $3905 \AA$ line. When possible, we avoid using this line as an abundance indicator and instead prefer the high-excitation $(4.90 \mathrm{eV} \leqslant \mathrm{EP} \leqslant 5.06 \mathrm{eV}) \mathrm{Si}$ I lines at $5665,5701,5708$, and $5772 \AA$. Our analysis reveals that the low excitation Si I 3905 and $4102 \AA$ lines $(\mathrm{EP}=1.91 \mathrm{eV})$ give consistent results in the three stars where both can be reliably measured $(\Delta=0.00 \pm 0.04, \sigma=0.07)$. In the nine stars with at least one high-excitation line and at least one low excitation line used as abundance indicators, the high-excitation lines yield abundances higher by $0.14 \pm 0.05 \operatorname{dex}(\sigma=0.15)$ on average. Here, we adopt the convention to correct the abundances of the low excitation lines in all stars by +0.14 dex to match the average abundance of the high-excitation lines. We do not include the low excitation lines in the reported mean silicon abundance for the 12 stars where at least one high-excitation line is also used. This correction accounts for the higher silicon abundances reported for the more metal-poor stars in our sample when compared with other recent investigations.

Previous studies of Mn I lines in late-type stars have demonstrated that the three Mn I resonance lines at 4030, 4033, and $4034 \AA$ yield abundances in LTE that are several tenths of a dex lower than the high-excitation neutral or singly ionized lines (e.g., Cayrel et al. 2004; Roederer et al. 2010). We thus take the following approach to identifying line-by-line systematic offsets in our data set. First, we identify any offsets among the high-excitation neutral lines from the mean of all highexcitation neutral lines within a given star. Then, we recompute the mean manganese abundance derived from the corrected high-excitation lines for each star. Finally, we identify any offsets among the neutral resonance lines relative to the corrected mean. This forces the resonance lines, on average, to have the same mean abundance as the high-excitation lines. Fortuitously, the mean $[\mathrm{Mn} / \mathrm{Fe}]$ ratio derived from neutral lines shows only a small difference from the mean $[\mathrm{Mn} / \mathrm{Fe}]$ ratio derived from the ionized lines, with a mean difference (ion minus neutral) of only $-0.038 \pm 0.011(\sigma=0.14)$. This level of agreement, not enforced by our method, is encouraging.

For $\mathrm{Cu}$ I, Tb II, Tm II, Ir I, and Th II, there are not enough stars with two or more lines measured to assess systematic offsets. For Co I, Y II, Ba II, and La II, there are not enough stars with two or more lines measured to assess systematic offsets in the MS class; in these cases, we adopt the corrections from stars in the SG class for the stars in the MS class. For Co I, a similar situation exists for stars in the HB class, and we also adopt the corrections from the stars in the SG class for stars in the HB class. For K I, Ce II, Pr II, Nd II, Sm II, Eu II, Gd II, Dy II, and Er II we adopt a single correction for stars in all evolutionary states. There are never enough stars in the BS class to define a separate set of corrections, so the abundances in these stars are corrected by adopting the offsets found for the stars in the SG class.

\subsection{Comparison with Previous Studies}

\subsubsection{Differences in the $[X / F e]$ Ratios}

We compare our derived $[\mathrm{X} / \mathrm{Fe}]$ ratios, where $\mathrm{X}$ is a given element, with those derived by previous studies. In particular, we focus on the 18 red giants in common with the First Stars analysis. To keep the comparisons manageable when comparing with other studies, we limit ourselves to two other studies that also examined large numbers of stars in common with the First Stars analysis, those of McWilliam et al. (1995a) and Yong et al. (2013). Note that Yong et al. rederived abundances of these stars from published EW values. We also compare with the 
Table 16

Line Abundance Corrections and Uncertainties

\begin{tabular}{|c|c|c|c|c|c|c|c|c|c|}
\hline \multirow[t]{2}{*}{ Species } & \multirow{2}{*}{$\begin{array}{c}\text { Wavelength } \\
(\AA ̊)\end{array}$} & \multicolumn{2}{|c|}{ RG } & \multicolumn{2}{|c|}{ SG } & \multicolumn{2}{|c|}{$\mathrm{HB}$} & \multicolumn{2}{|c|}{ MS } \\
\hline & & Correction & $\sigma$ & Correction & $\sigma$ & Correction & $\sigma$ & Correction & $\sigma$ \\
\hline O I & 7771.94 & -0.50 & 0.25 & -0.50 & 0.25 & -0.50 & 0.25 & -0.50 & 0.25 \\
\hline OI & 7774.17 & -0.50 & 0.25 & -0.50 & 0.25 & -0.50 & 0.25 & -0.50 & 0.25 \\
\hline OI & 7775.39 & -0.50 & 0.25 & -0.50 & 0.25 & -0.50 & 0.25 & -0.50 & 0.25 \\
\hline $\mathrm{NaI}$ & 5682.63 & +0.00 & 0.13 & +0.00 & 0.13 & +0.00 & 0.13 & +0.00 & 0.13 \\
\hline $\mathrm{Na}$ & 5688.20 & +0.00 & 0.13 & +0.00 & 0.13 & +0.00 & 0.13 & +0.00 & 0.13 \\
\hline $\mathrm{NaI}$ & 5889.95 & +0.01 & 0.06 & +0.01 & 0.06 & +0.01 & 0.06 & +0.01 & 0.06 \\
\hline $\mathrm{NaI}$ & 5895.92 & -0.01 & 0.06 & -0.01 & 0.06 & -0.01 & 0.06 & -0.01 & 0.06 \\
\hline $\mathrm{Mg}_{\mathrm{I}}$ & 3829.36 & +0.04 & 0.08 & +0.19 & 0.07 & +0.04 & 0.08 & $\ldots$ & $\ldots$ \\
\hline $\mathrm{Mg}_{\mathrm{I}}$ & 3832.30 & +0.14 & 0.09 & +0.34 & 0.07 & $\ldots$ & $\ldots$ & $\ldots$ & $\ldots$ \\
\hline $\mathrm{Mg}_{\mathrm{I}}$ & 3838.29 & +0.18 & 0.10 & +0.44 & 0.04 & $\ldots$ & $\ldots$ & $\ldots$ & $\ldots$ \\
\hline $\mathrm{Mg}_{\mathrm{I}}$ & 4057.51 & +0.02 & 0.07 & -0.01 & 0.07 & +0.02 & 0.09 & -0.01 & 0.16 \\
\hline $\mathrm{Mg}_{\mathrm{I}}$ & 4167.27 & -0.05 & 0.09 & -0.02 & 0.05 & +0.04 & 0.07 & -0.11 & 0.07 \\
\hline $\operatorname{Mg}_{I}$ & 4702.99 & +0.06 & 0.04 & +0.05 & 0.06 & +0.12 & 0.05 & +0.10 & 0.10 \\
\hline $\mathrm{Mg}_{\mathrm{I}}$ & 5172.68 & -0.05 & 0.07 & -0.08 & 0.08 & -0.20 & 0.11 & -0.11 & 0.04 \\
\hline $\mathrm{Mg}_{\mathrm{I}}$ & 5183.60 & -0.10 & 0.07 & -0.09 & 0.08 & -0.35 & 0.13 & -0.13 & 0.09 \\
\hline $\mathrm{Mg}_{\mathrm{I}}$ & 5528.40 & -0.05 & 0.08 & -0.05 & 0.05 & -0.02 & 0.05 & -0.01 & 0.08 \\
\hline $\mathrm{Mg}_{\mathrm{I}}$ & 5711.09 & -0.02 & 0.13 & -0.02 & 0.08 & -0.06 & 0.08 & +0.00 & 0.06 \\
\hline $\mathrm{Al}$ I & 3944.00 & -0.04 & 0.09 & -0.04 & 0.08 & -0.01 & 0.10 & $\ldots$ & $\ldots$ \\
\hline $\mathrm{Al}$ I & 3961.52 & +0.04 & 0.09 & +0.04 & 0.08 & +0.01 & 0.10 & $\ldots$ & $\ldots$ \\
\hline Si I & 3905.52 & +0.14 & 0.16 & +0.14 & 0.16 & +0.14 & 0.16 & +0.14 & 0.16 \\
\hline Si I & 4102.94 & +0.14 & 0.16 & +0.14 & 0.16 & +0.14 & 0.16 & +0.14 & 0.16 \\
\hline Si I & 5665.55 & +0.02 & 0.11 & +0.02 & 0.11 & +0.02 & 0.11 & +0.02 & 0.11 \\
\hline Si I & 5701.10 & +0.02 & 0.11 & +0.02 & 0.11 & +0.02 & 0.11 & +0.02 & 0.11 \\
\hline Si I & 5708.40 & +0.00 & 0.11 & +0.00 & 0.11 & +0.00 & 0.11 & +0.00 & 0.11 \\
\hline Si I & 5772.15 & -0.04 & 0.13 & -0.04 & 0.13 & -0.04 & 0.13 & -0.04 & 0.13 \\
\hline $\mathrm{K}_{\mathrm{I}}$ & 7664.90 & +0.01 & 0.09 & +0.01 & 0.09 & +0.01 & 0.09 & +0.01 & 0.09 \\
\hline $\mathrm{K}_{\mathrm{I}}$ & 7698.96 & -0.01 & 0.09 & -0.01 & 0.09 & -0.01 & 0.09 & -0.01 & 0.09 \\
\hline $\mathrm{CaI}$ & 4226.73 & +0.18 & 0.07 & +0.06 & 0.13 & -0.23 & 0.06 & $\ldots$ & $\ldots$ \\
\hline $\mathrm{CaI}$ & 4283.01 & -0.09 & 0.08 & +0.01 & 0.04 & +0.03 & 0.05 & +0.01 & 0.06 \\
\hline $\mathrm{Ca}$ & 4318.65 & +0.07 & 0.04 & +0.05 & 0.04 & +0.08 & 0.04 & +0.11 & 0.05 \\
\hline $\mathrm{Ca}$ & 4425.44 & +0.05 & 0.07 & +0.05 & 0.05 & +0.09 & 0.03 & +0.07 & 0.07 \\
\hline $\mathrm{Ca}$ & 4434.96 & +0.01 & 0.06 & +0.02 & 0.04 & +0.05 & 0.07 & -0.01 & 0.02 \\
\hline $\mathrm{Ca}$ & 4435.69 & +0.02 & 0.05 & +0.05 & 0.06 & +0.00 & 0.05 & +0.05 & 0.06 \\
\hline $\mathrm{CaI}$ & 4454.78 & +0.10 & 0.06 & +0.08 & 0.04 & +0.09 & 0.04 & +0.10 & 0.05 \\
\hline $\mathrm{Ca} I$ & 4455.89 & +0.05 & 0.06 & +0.07 & 0.06 & +0.03 & 0.12 & +0.09 & 0.05 \\
\hline $\mathrm{Ca} I$ & 5588.76 & -0.05 & 0.05 & -0.09 & 0.08 & -0.05 & 0.05 & -0.11 & 0.04 \\
\hline $\mathrm{CaI}$ & 5857.45 & -0.05 & 0.11 & -0.06 & 0.07 & -0.05 & 0.06 & -0.07 & 0.07 \\
\hline $\mathrm{Ca} I$ & 6102.72 & -0.06 & 0.07 & -0.08 & 0.08 & -0.14 & 0.07 & -0.06 & 0.04 \\
\hline $\mathrm{Ca} I$ & 6122.21 & -0.10 & 0.04 & -0.08 & 0.06 & -0.09 & 0.05 & -0.09 & 0.03 \\
\hline $\mathrm{Ca} I$ & 6162.17 & -0.07 & 0.02 & -0.07 & 0.06 & -0.07 & 0.04 & -0.07 & 0.04 \\
\hline $\mathrm{Ca}$ & 6439.07 & +0.09 & 0.05 & +0.07 & 0.06 & +0.10 & 0.03 & +0.07 & 0.07 \\
\hline Sc II & 3576.34 & -0.10 & 0.14 & -0.10 & 0.12 & -0.21 & 0.21 & $\ldots$ & $\ldots$ \\
\hline Sc II & 3590.47 & -0.23 & 0.17 & -0.04 & 0.11 & -0.13 & 0.14 & $\ldots$ & $\ldots$ \\
\hline Sc II & 3645.31 & -0.14 & 0.12 & +0.00 & 0.06 & -0.11 & 0.10 & $\ldots$ & $\ldots$ \\
\hline $\mathrm{Sc}_{\text {II }}$ & 4246.82 & +0.08 & 0.10 & -0.04 & 0.09 & -0.06 & 0.09 & +0.04 & 0.03 \\
\hline Sc II & 4400.39 & +0.03 & 0.04 & +0.00 & 0.04 & +0.05 & 0.05 & +0.00 & 0.02 \\
\hline $\mathrm{Sc}_{\text {II }}$ & 4415.54 & +0.03 & 0.04 & +0.01 & 0.05 & +0.06 & 0.03 & +0.01 & 0.03 \\
\hline Sc II & 4670.41 & -0.02 & 0.04 & +0.02 & 0.05 & +0.03 & 0.06 & +0.04 & 0.02 \\
\hline $\mathrm{Sc}_{\text {II }}$ & 5526.79 & +0.01 & 0.05 & +0.03 & 0.05 & +0.01 & 0.06 & +0.02 & 0.04 \\
\hline $\mathrm{Sc}_{\mathrm{II}}$ & 5657.91 & -0.03 & 0.07 & -0.02 & 0.03 & -0.03 & 0.10 & -0.08 & 0.02 \\
\hline Ti I & 3989.76 & -0.01 & 0.10 & -0.09 & 0.06 & -0.05 & 0.18 & -0.06 & 0.02 \\
\hline Ti I & 3998.64 & +0.05 & 0.08 & -0.01 & 0.07 & +0.08 & 0.09 & +0.02 & 0.03 \\
\hline Ti I & 4512.73 & -0.02 & 0.09 & +0.03 & 0.04 & +0.00 & 0.07 & +0.01 & 0.04 \\
\hline Ti I & 4518.02 & -0.01 & 0.10 & -0.01 & 0.05 & -0.02 & 0.02 & +0.01 & 0.04 \\
\hline Ti I & 4533.24 & +0.03 & 0.05 & -0.01 & 0.04 & +0.05 & 0.07 & +0.01 & 0.04 \\
\hline Ti I & 4534.78 & +0.04 & 0.04 & +0.01 & 0.05 & +0.07 & 0.08 & +0.03 & 0.02 \\
\hline Ti I & 4548.76 & +0.02 & 0.08 & +0.03 & 0.07 & +0.02 & 0.11 & +0.03 & 0.03 \\
\hline Ti I & 4555.48 & +0.02 & 0.08 & +0.05 & 0.08 & -0.03 & 0.10 & +0.05 & 0.02 \\
\hline Ti I & 4656.47 & +0.01 & 0.08 & +0.01 & 0.06 & -0.02 & 0.15 & +0.00 & 0.02 \\
\hline Ti I & 4681.91 & +0.01 & 0.06 & +0.01 & 0.11 & +0.01 & 0.05 & +0.00 & 0.03 \\
\hline Ti I & 4840.87 & -0.02 & 0.11 & +0.02 & 0.06 & -0.02 & 0.18 & +0.04 & 0.06 \\
\hline Ti I & 4981.73 & +0.03 & 0.06 & +0.02 & 0.07 & +0.07 & 0.12 & +0.03 & 0.02 \\
\hline Ti I & 4991.07 & -0.03 & 0.07 & -0.06 & 0.11 & -0.02 & 0.07 & -0.08 & 0.04 \\
\hline Ti I & 4999.50 & -0.02 & 0.10 & -0.01 & 0.06 & -0.03 & 0.08 & -0.04 & 0.06 \\
\hline Ti I & 5016.16 & -0.04 & 0.10 & +0.01 & 0.02 & +0.03 & 0.04 & -0.01 & 0.10 \\
\hline
\end{tabular}


Table 16

(Continued)

\begin{tabular}{|c|c|c|c|c|c|c|c|c|c|}
\hline \multirow[t]{2}{*}{ Species } & \multirow{2}{*}{ 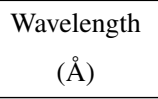 } & \multicolumn{2}{|c|}{ RG } & \multicolumn{2}{|c|}{ SG } & \multicolumn{2}{|c|}{$\mathrm{HB}$} & \multicolumn{2}{|c|}{ MS } \\
\hline & & Correction & $\sigma$ & Correction & $\sigma$ & Correction & $\sigma$ & Correction & $\sigma$ \\
\hline Ti I & 5064.65 & -0.05 & 0.08 & -0.03 & 0.06 & -0.07 & 0.02 & -0.04 & 0.02 \\
\hline Ti I & 5173.74 & -0.01 & 0.08 & -0.03 & 0.14 & -0.03 & 0.04 & $\ldots$ & \\
\hline Ti I & 5192.97 & -0.03 & 0.09 & -0.01 & 0.09 & -0.04 & 0.06 & -0.03 & 0.02 \\
\hline Ti I & 5210.38 & +0.01 & 0.09 & +0.00 & 0.06 & -0.03 & 0.07 & -0.14 & 0.09 \\
\hline Ti II & 3372.79 & $\ldots$ & $\ldots$ & +0.05 & 0.18 & $\ldots$ & $\ldots$ & $\ldots$ & $\ldots$ \\
\hline Ti II & 3387.83 & +0.00 & 0.15 & -0.03 & 0.11 & $\ldots$ & $\ldots$ & $\ldots$ & $\ldots$ \\
\hline Ti II & 3394.57 & -0.02 & 0.20 & -0.08 & 0.11 & $\ldots$ & $\ldots$ & $\ldots$ & $\ldots$ \\
\hline Ti II & 3456.38 & +0.24 & 0.07 & +0.21 & 0.11 & $\ldots$ & $\ldots$ & $\ldots$ & $\ldots$ \\
\hline Ti II & 3477.18 & $\ldots$ & $\ldots$ & -0.17 & 0.05 & $\ldots$ & $\ldots$ & $\ldots$ & $\ldots$ \\
\hline Ti II & 3489.74 & -0.23 & 0.13 & -0.10 & 0.13 & $\ldots$ & $\ldots$ & $\ldots$ & $\ldots$ \\
\hline Ti II & 3491.05 & -0.11 & 0.14 & -0.05 & 0.09 & $\ldots$ & $\ldots$ & $\ldots$ & .. \\
\hline Ti II & 3759.29 & -0.01 & 0.07 & -0.04 & 0.05 & $\ldots$ & $\ldots$ & $\ldots$ & .. \\
\hline Ti II & 3761.32 & -0.04 & 0.08 & +0.00 & 0.08 & $\ldots$ & $\ldots$ & +0.02 & 0.12 \\
\hline Ti II & 3913.46 & -0.13 & 0.11 & -0.11 & 0.09 & $\ldots$ & $\ldots$ & +0.06 & 0.04 \\
\hline Ti II & 4028.34 & +0.06 & 0.11 & +0.06 & 0.07 & +0.08 & 0.06 & -0.04 & 0.11 \\
\hline Ti II & 4337.91 & +0.05 & 0.08 & $\ldots$ & & $\ldots$ & $\ldots$ & $\ldots$ & \\
\hline Ti II & 4394.06 & +0.01 & 0.05 & +0.05 & 0.08 & +0.01 & 0.04 & -0.04 & 0.08 \\
\hline Ti II & 4395.03 & -0.09 & 0.09 & -0.08 & 0.10 & -0.18 & 0.12 & -0.06 & 0.11 \\
\hline Ti II & 4395.84 & +0.09 & 0.07 & +0.12 & 0.05 & +0.06 & 0.03 & +0.13 & 0.12 \\
\hline Ti II & 4398.29 & +0.23 & 0.07 & $\ldots$ & $\ldots$ & $\ldots$ & $\ldots$ & $\ldots$ & $\ldots$ \\
\hline Ti II & 4399.77 & -0.04 & 0.08 & -0.02 & 0.10 & +0.05 & 0.05 & -0.07 & 0.15 \\
\hline Ti II & 4409.52 & +0.09 & 0.05 & $\ldots$ & $\ldots$ & +0.02 & 0.13 & $\ldots$ & $\ldots$ \\
\hline Ti II & 4417.71 & -0.03 & 0.07 & -0.03 & 0.08 & +0.02 & 0.03 & -0.01 & 0.08 \\
\hline Ti II & 4418.33 & +0.03 & 0.06 & +0.04 & 0.07 & +0.02 & 0.06 & -0.06 & 0.04 \\
\hline Ti II & 4443.80 & -0.02 & 0.09 & -0.13 & 0.09 & -0.06 & 0.06 & -0.01 & 0.06 \\
\hline Ti II & 4444.55 & +0.03 & 0.05 & +0.08 & 0.08 & +0.05 & 0.05 & +0.08 & 0.05 \\
\hline Ti II & 4450.48 & -0.04 & 0.07 & -0.03 & 0.07 & -0.01 & 0.04 & -0.01 & 0.09 \\
\hline Ti II & 4464.45 & -0.01 & 0.04 & +0.04 & 0.05 & +0.01 & 0.02 & -0.03 & 0.04 \\
\hline Ti II & 4470.85 & +0.13 & 0.06 & +0.16 & 0.05 & +0.14 & 0.04 & +0.12 & 0.06 \\
\hline Ti II & 4493.52 & +0.10 & 0.08 & +0.16 & 0.10 & +0.11 & 0.12 & +0.20 & 0.05 \\
\hline Ti II & 4501.27 & -0.06 & 0.08 & -0.15 & 0.08 & -0.05 & 0.06 & -0.07 & 0.07 \\
\hline Ti II & 4533.96 & -0.05 & 0.11 & -0.10 & 0.11 & -0.08 & 0.07 & -0.06 & 0.09 \\
\hline Ti II & 4571.97 & +0.03 & 0.12 & -0.09 & 0.11 & -0.02 & 0.07 & -0.09 & 0.10 \\
\hline Ti II & 4583.41 & -0.01 & 0.12 & +0.10 & 0.10 & -0.10 & 0.12 & +0.04 & 0.06 \\
\hline Ti II & 4636.32 & +0.13 & 0.08 & $\ldots$ & $\ldots$ & $\ldots$ & $\ldots$ & $\ldots$ & $\ldots$ \\
\hline Ti II & 4657.20 & +0.01 & 0.07 & +0.04 & 0.06 & +0.00 & 0.00 & +0.07 & 0.06 \\
\hline Ti II & 4708.66 & -0.02 & 0.08 & +0.08 & 0.05 & +0.03 & 0.08 & +0.05 & 0.07 \\
\hline Ti II & 4798.53 & -0.04 & 0.08 & +0.04 & 0.10 & -0.13 & 0.11 & +0.04 & 0.09 \\
\hline Ti II & 5129.16 & -0.01 & 0.11 & -0.02 & 0.10 & +0.02 & 0.10 & -0.04 & 0.05 \\
\hline Ti II & 5185.90 & +0.08 & 0.09 & +0.05 & 0.08 & +0.11 & 0.11 & $\ldots$ & $\ldots$ \\
\hline Ti II & 5188.69 & -0.08 & 0.12 & -0.10 & 0.10 & -0.03 & 0.10 & -0.16 & 0.13 \\
\hline Ti II & 5226.54 & -0.01 & 0.06 & +0.00 & 0.07 & +0.05 & 0.06 & -0.04 & 0.03 \\
\hline Ti II & 5336.79 & +0.02 & 0.06 & +0.07 & 0.06 & +0.08 & 0.05 & +0.01 & 0.06 \\
\hline Ti II & 5381.02 & -0.02 & 0.08 & +0.03 & 0.06 & -0.04 & 0.05 & +0.01 & 0.04 \\
\hline Ti II & 5418.77 & -0.02 & 0.08 & -0.01 & 0.10 & +0.07 & 0.03 & +0.00 & 0.05 \\
\hline $\mathrm{V}_{\text {II }}$ & 3951.96 & +0.06 & 0.09 & +0.04 & 0.12 & +0.04 & 0.10 & +0.03 & 0.17 \\
\hline $\mathrm{V}_{\text {II }}$ & 4005.71 & -0.06 & 0.09 & -0.04 & 0.12 & -0.04 & 0.10 & -0.03 & 0.17 \\
\hline Cr I & 3578.68 & +0.11 & 0.14 & -0.06 & 0.18 & -0.02 & 0.13 & $\ldots$ & $\ldots$ \\
\hline CrI & 4274.80 & +0.01 & 0.11 & -0.09 & 0.09 & -0.01 & 0.14 & $\ldots$ & $\ldots$ \\
\hline Cr I & 4289.72 & +0.10 & 0.17 & +0.00 & 0.08 & +0.18 & 0.13 & +0.07 & 0.05 \\
\hline Cr I & 4545.95 & -0.01 & 0.07 & +0.00 & 0.06 & -0.02 & 0.13 & +0.03 & 0.08 \\
\hline Cr I & 4646.15 & +0.01 & 0.04 & -0.03 & 0.03 & +0.03 & 0.05 & -0.02 & 0.03 \\
\hline CrI & 4651.28 & -0.03 & 0.12 & +0.00 & 0.06 & -0.04 & 0.04 & -0.02 & 0.07 \\
\hline CrI & 4789.34 & -0.06 & 0.08 & -0.06 & 0.12 & $\ldots$ & $\ldots$ & -0.02 & 0.05 \\
\hline $\mathrm{Cr} \mathrm{I}$ & 5206.04 & +0.00 & 0.08 & -0.07 & 0.08 & -0.04 & 0.14 & +0.00 & 0.06 \\
\hline Cr I & 5296.69 & -0.03 & 0.07 & +0.04 & 0.08 & -0.06 & 0.15 & +0.00 & 0.03 \\
\hline $\mathrm{Cr} \mathrm{I}$ & 5298.28 & -0.06 & 0.06 & -0.01 & 0.05 & -0.05 & 0.06 & -0.06 & 0.09 \\
\hline $\mathrm{CrI}$ & 5300.74 & +0.10 & 0.05 & +0.01 & 0.11 & $\ldots$ & $\ldots$ & +0.09 & 0.05 \\
\hline Cr I & 5345.80 & -0.01 & 0.07 & +0.02 & 0.04 & -0.02 & 0.09 & -0.04 & 0.06 \\
\hline Cr I & 5348.31 & +0.05 & 0.07 & +0.06 & 0.06 & +0.02 & 0.11 & +0.00 & 0.04 \\
\hline CrI & 5409.77 & +0.00 & 0.06 & +0.01 & 0.06 & +0.01 & 0.04 & -0.06 & 0.11 \\
\hline Cr II & 3408.74 & +0.09 & 0.11 & +0.08 & 0.20 & $\ldots$ & $\ldots$ & $\ldots$ & $\ldots$ \\
\hline $\mathrm{Cr}$ II & 4558.59 & -0.03 & 0.07 & -0.03 & 0.05 & -0.05 & 0.06 & -0.02 & 0.07 \\
\hline $\mathrm{Cr}$ II & 4588.14 & +0.04 & 0.05 & +0.01 & 0.05 & +0.00 & 0.04 & +0.03 & 0.09 \\
\hline $\mathrm{Cr}$ II & 4591.99 & -0.03 & 0.07 & +0.03 & 0.07 & +0.06 & 0.09 & -0.02 & 0.08 \\
\hline
\end{tabular}


Table 16

(Continued)

\begin{tabular}{|c|c|c|c|c|c|c|c|c|c|}
\hline \multirow[t]{2}{*}{ Species } & \multirow{2}{*}{ 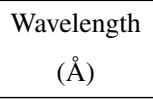 } & \multicolumn{2}{|c|}{ RG } & \multicolumn{2}{|c|}{ SG } & \multicolumn{2}{|c|}{$\mathrm{HB}$} & \multicolumn{2}{|c|}{ MS } \\
\hline & & Correction & $\sigma$ & Correction & $\sigma$ & Correction & $\sigma$ & Correction & $\sigma$ \\
\hline Mn I & 4030.75 & +0.15 & 0.19 & +0.11 & 0.25 & +0.33 & 0.22 & -0.12 & 0.19 \\
\hline Mn I & 4033.06 & +0.38 & 0.13 & +0.23 & 0.17 & +0.40 & 0.14 & +0.07 & 0.08 \\
\hline Mn I & 4034.48 & +0.38 & 0.09 & +0.20 & 0.14 & +0.33 & 0.14 & -0.02 & 0.27 \\
\hline Mn I & 4041.35 & +0.04 & 0.06 & +0.01 & 0.08 & +0.02 & 0.06 & -0.01 & 0.11 \\
\hline Mn I & 4754.04 & +0.00 & 0.06 & +0.02 & 0.09 & -0.01 & 0.05 & +0.07 & 0.09 \\
\hline Mn I & 4762.37 & -0.06 & 0.07 & -0.08 & 0.08 & -0.06 & 0.07 & -0.01 & 0.14 \\
\hline Mn I & 4823.52 & -0.01 & 0.06 & +0.02 & 0.08 & +0.02 & 0.03 & -0.03 & 0.07 \\
\hline Mn I & 6021.82 & -0.03 & 0.08 & -0.03 & 0.05 & $\ldots$ & $\ldots$ & -0.05 & 0.06 \\
\hline Mn II & 3441.99 & +0.01 & 0.10 & +0.00 & 0.14 & -0.09 & 0.07 & +0.03 & 0.05 \\
\hline Mn II & 3460.32 & +0.05 & 0.12 & +0.01 & 0.09 & +0.05 & 0.04 & -0.01 & 0.05 \\
\hline Mn II & 3482.90 & +0.06 & 0.11 & +0.01 & 0.10 & +0.01 & 0.11 & +0.01 & 0.08 \\
\hline Mn II & 3488.68 & -0.05 & 0.12 & -0.02 & 0.08 & +0.05 & 0.17 & +0.00 & 0.11 \\
\hline Mn II & 3497.53 & -0.11 & 0.09 & -0.03 & 0.08 & -0.06 & 0.09 & -0.05 & 0.15 \\
\hline Co I & 3409.18 & +0.11 & 0.05 & $\ldots$ & $\ldots$ & $\ldots$ & $\ldots$ & $\ldots$ & $\ldots$ \\
\hline Co I & 3412.34 & -0.09 & 0.16 & +0.05 & 0.15 & +0.05 & 0.15 & +0.05 & 0.15 \\
\hline Co I & 3412.63 & -0.24 & 0.09 & -0.18 & 0.14 & -0.18 & 0.14 & -0.18 & 0.14 \\
\hline Co I & 3449.44 & +0.10 & 0.06 & +0.02 & 0.03 & +0.02 & 0.03 & +0.02 & 0.03 \\
\hline CoI & 3502.28 & +0.38 & 0.13 & +0.15 & 0.09 & +0.15 & 0.09 & +0.15 & 0.09 \\
\hline CoI & 3518.34 & +0.19 & 0.07 & +0.12 & 0.10 & +0.12 & 0.10 & +0.12 & 0.10 \\
\hline Co I & 3521.57 & -0.06 & 0.08 & -0.04 & 0.04 & -0.04 & 0.04 & -0.04 & 0.04 \\
\hline Co I & 3529.03 & +0.07 & 0.11 & -0.05 & 0.07 & -0.05 & 0.07 & -0.05 & 0.07 \\
\hline Co I & 3842.05 & -0.10 & 0.08 & -0.10 & 0.06 & -0.10 & 0.06 & -0.10 & 0.06 \\
\hline CoI & 3845.47 & -0.02 & 0.07 & -0.02 & 0.05 & -0.02 & 0.05 & -0.02 & 0.05 \\
\hline CoI & 3894.08 & +0.07 & 0.07 & +0.10 & 0.02 & +0.10 & 0.02 & +0.10 & 0.02 \\
\hline CoI & 4121.32 & -0.11 & 0.07 & -0.02 & 0.04 & -0.02 & 0.04 & -0.02 & 0.04 \\
\hline Ni I & 3437.28 & -0.05 & 0.14 & +0.01 & 0.12 & $\ldots$ & $\ldots$ & $\ldots$ & $\ldots$ \\
\hline $\mathrm{Ni} I$ & 3452.89 & +0.02 & 0.15 & -0.07 & 0.11 & $\ldots$ & $\ldots$ & $\ldots$ & $\ldots$ \\
\hline $\mathrm{Ni} I$ & 3472.54 & +0.13 & 0.13 & -0.05 & 0.13 & $\ldots$ & $\ldots$ & $\ldots$ & $\ldots$ \\
\hline Ni I & 3492.96 & +0.20 & 0.11 & +0.04 & 0.12 & $\ldots$ & $\ldots$ & $\ldots$ & $\ldots$ \\
\hline $\mathrm{Ni} I$ & 3500.85 & -0.02 & 0.15 & -0.08 & 0.12 & -0.02 & 0.20 & -0.07 & 0.23 \\
\hline $\mathrm{Ni}$ I & 3519.77 & +0.01 & 0.14 & +0.06 & 0.08 & $\ldots$ & $\ldots$ & $\ldots$ & $\ldots$ \\
\hline $\mathrm{Ni}$ I & 3524.54 & +0.11 & 0.08 & +0.06 & 0.14 & $\ldots$ & $\ldots$ & $\ldots$ & $\ldots$ \\
\hline $\mathrm{Ni} I$ & 3597.70 & +0.14 & 0.15 & +0.03 & 0.09 & +0.09 & 0.07 & +0.05 & 0.28 \\
\hline Ni I & 3783.53 & +0.03 & 0.13 & +0.08 & 0.07 & $\ldots$ & $\ldots$ & +0.25 & 0.14 \\
\hline Ni I & 3807.14 & +0.15 & 0.14 & +0.08 & 0.09 & +0.19 & 0.13 & +0.20 & 0.16 \\
\hline Ni I & 4605.00 & +0.02 & 0.08 & +0.04 & 0.09 & +0.07 & 0.04 & +0.05 & 0.02 \\
\hline Ni I & 4686.22 & +0.07 & 0.04 & -0.01 & 0.06 & $\ldots$ & $\ldots$ & -0.01 & 0.12 \\
\hline $\mathrm{Ni}$ I & 4904.41 & -0.05 & 0.09 & -0.04 & 0.09 & +0.00 & 0.05 & +0.00 & 0.04 \\
\hline $\mathrm{Ni} I$ & 5081.11 & -0.04 & 0.08 & -0.01 & 0.10 & +0.01 & 0.09 & +0.06 & 0.07 \\
\hline Ni I & 5084.08 & +0.04 & 0.08 & +0.06 & 0.10 & +0.02 & 0.06 & +0.13 & 0.04 \\
\hline Ni I & 5115.40 & -0.02 & 0.11 & -0.02 & 0.06 & +0.04 & 0.08 & $\ldots$ & $\ldots$ \\
\hline Ni I & 5155.76 & +0.02 & 0.06 & -0.03 & 0.06 & $\ldots$ & $\ldots$ & -0.02 & 0.12 \\
\hline Ni I & 5476.91 & -0.06 & 0.09 & -0.14 & 0.09 & -0.12 & 0.05 & -0.23 & 0.12 \\
\hline $\mathrm{Ni}$ I & 6643.64 & -0.05 & 0.09 & -0.05 & 0.05 & -0.02 & 0.04 & -0.09 & 0.08 \\
\hline Ni I & 6767.77 & -0.05 & 0.06 & -0.02 & 0.04 & +0.01 & 0.02 & -0.11 & 0.03 \\
\hline $\mathrm{Zn} \mathrm{I}$ & 4680.14 & -0.07 & 0.09 & -0.05 & 0.10 & -0.05 & 0.12 & $\ldots$ & $\ldots$ \\
\hline Zn I & 4722.16 & +0.04 & 0.12 & +0.01 & 0.16 & -0.02 & 0.15 & +0.00 & 0.10 \\
\hline $\mathrm{Zn}$ I & 4810.54 & +0.08 & 0.12 & +0.04 & 0.16 & +0.06 & 0.15 & +0.00 & 0.10 \\
\hline Sr II & 4077.71 & +0.00 & 0.12 & +0.00 & 0.12 & -0.01 & 0.16 & +0.03 & 0.14 \\
\hline Sr II & 4215.52 & +0.00 & 0.12 & +0.00 & 0.12 & +0.01 & 0.16 & -0.03 & 0.14 \\
\hline$Y_{\text {II }}$ & 3600.73 & +0.01 & 0.11 & -0.06 & 0.19 & -0.05 & 0.15 & -0.06 & 0.19 \\
\hline$Y_{\text {II }}$ & 3774.33 & -0.01 & 0.12 & -0.05 & 0.08 & +0.00 & 0.13 & -0.05 & 0.08 \\
\hline$Y_{\text {II }}$ & 4398.01 & +0.08 & 0.08 & +0.16 & 0.05 & +0.10 & 0.07 & +0.16 & 0.05 \\
\hline $\mathrm{Y}_{\mathrm{II}}$ & 4883.68 & -0.02 & 0.06 & +0.01 & 0.07 & -0.04 & 0.06 & +0.01 & 0.07 \\
\hline$Y_{\text {II }}$ & 5087.42 & -0.06 & 0.07 & -0.01 & 0.06 & -0.09 & 0.08 & -0.01 & 0.06 \\
\hline$Y_{\text {II }}$ & 5200.41 & -0.01 & 0.09 & +0.06 & 0.06 & +0.02 & 0.09 & +0.06 & 0.06 \\
\hline$Y_{\text {II }}$ & 5205.72 & -0.05 & 0.04 & -0.09 & 0.06 & $\ldots$ & $\ldots$ & -0.09 & 0.06 \\
\hline $\mathrm{Zr}$ II & 4149.20 & -0.04 & 0.12 & -0.04 & 0.12 & -0.03 & 0.12 & -0.04 & 0.10 \\
\hline Zr II & 4161.20 & +0.03 & 0.12 & +0.00 & 0.12 & -0.01 & 0.12 & +0.03 & 0.10 \\
\hline Zr II & 4208.98 & +0.00 & 0.12 & +0.05 & 0.12 & +0.05 & 0.12 & +0.01 & 0.10 \\
\hline Ba II & 4554.03 & +0.05 & 0.11 & +0.01 & 0.09 & -0.04 & 0.17 & +0.01 & 0.09 \\
\hline Ba II & 5853.68 & +0.11 & 0.05 & +0.08 & 0.09 & +0.16 & 0.13 & +0.08 & 0.09 \\
\hline Ba II & 6141.71 & +0.00 & 0.06 & -0.02 & 0.06 & -0.07 & 0.11 & -0.02 & 0.06 \\
\hline Ba II & 6496.90 & -0.13 & 0.04 & -0.10 & 0.05 & -0.22 & 0.12 & -0.10 & 0.05 \\
\hline La II & 3988.51 & +0.04 & 0.05 & +0.04 & 0.06 & +0.02 & 0.03 & +0.04 & 0.06 \\
\hline
\end{tabular}




\begin{tabular}{|c|c|c|c|c|c|c|c|c|c|}
\hline & & & & $\begin{array}{r}\text { Table 1 } \\
\text { (Continue }\end{array}$ & & & & & \\
\hline \multirow[t]{2}{*}{ Species } & \multirow{2}{*}{$\begin{array}{c}\text { Wavelength } \\
\text { (Å) }\end{array}$} & \multicolumn{2}{|c|}{ RG } & \multicolumn{2}{|c|}{ SG } & \multicolumn{2}{|c|}{ HB } & \multicolumn{2}{|c|}{ MS } \\
\hline & & Correction & $\sigma$ & Correction & $\sigma$ & Correction & $\sigma$ & Correction & $\sigma$ \\
\hline La II & 3995.74 & +0.05 & 0.04 & +0.05 & 0.05 & +0.00 & 0.05 & +0.05 & 0.05 \\
\hline La II & 4086.71 & -0.05 & 0.06 & -0.08 & 0.04 & -0.06 & 0.06 & -0.08 & 0.04 \\
\hline La II & 4123.22 & -0.01 & 0.05 & +0.01 & 0.06 & +0.06 & 0.06 & +0.01 & 0.06 \\
\hline Ce II & 3999.24 & +0.06 & 0.06 & +0.06 & 0.06 & +0.06 & 0.06 & +0.06 & 0.06 \\
\hline Ce II & 4073.47 & +0.04 & 0.03 & +0.04 & 0.03 & +0.04 & 0.03 & +0.04 & 0.03 \\
\hline Ce II & 4083.22 & -0.01 & 0.06 & -0.01 & 0.06 & -0.01 & 0.06 & -0.01 & 0.06 \\
\hline Ce II & 4418.78 & -0.05 & 0.08 & -0.05 & 0.08 & -0.05 & 0.08 & -0.05 & 0.08 \\
\hline Ce II & 4562.36 & -0.03 & 0.04 & -0.03 & 0.04 & -0.03 & 0.04 & -0.03 & 0.04 \\
\hline Pr II & 4062.80 & -0.13 & 0.20 & -0.13 & 0.20 & -0.13 & 0.20 & -0.13 & 0.20 \\
\hline Pr II & 4143.13 & +0.04 & 0.20 & +0.04 & 0.20 & +0.04 & 0.20 & +0.04 & 0.20 \\
\hline Pr II & 4179.40 & -0.10 & 0.20 & -0.10 & 0.20 & -0.10 & 0.20 & -0.10 & 0.20 \\
\hline Pr II & 4222.95 & +0.13 & 0.20 & +0.13 & 0.20 & +0.13 & 0.20 & +0.13 & 0.20 \\
\hline Pr II & 4408.81 & +0.05 & 0.20 & +0.05 & 0.20 & +0.05 & 0.20 & +0.05 & 0.20 \\
\hline Nd II & 4109.45 & -0.10 & 0.08 & -0.10 & 0.08 & -0.10 & 0.08 & -0.10 & 0.08 \\
\hline Nd II & 4446.38 & +0.10 & 0.06 & +0.10 & 0.06 & +0.10 & 0.06 & +0.10 & 0.06 \\
\hline Nd II & 4462.98 & -0.06 & 0.04 & -0.06 & 0.04 & -0.06 & 0.04 & -0.06 & 0.04 \\
\hline Nd II & 4706.54 & +0.01 & 0.04 & +0.01 & 0.04 & +0.01 & 0.04 & +0.01 & 0.04 \\
\hline Nd II & 4825.48 & +0.03 & 0.04 & +0.03 & 0.04 & +0.03 & 0.04 & +0.03 & 0.04 \\
\hline Sm II & 4424.34 & +0.05 & 0.10 & +0.05 & 0.10 & +0.05 & 0.10 & +0.05 & 0.10 \\
\hline Sm II & 4467.34 & -0.05 & 0.10 & -0.05 & 0.10 & -0.05 & 0.10 & -0.05 & 0.10 \\
\hline Eu II & 3819.67 & +0.02 & 0.05 & +0.02 & 0.05 & +0.02 & 0.05 & +0.02 & 0.05 \\
\hline Eu II & 3907.11 & +0.02 & 0.06 & +0.02 & 0.06 & +0.02 & 0.06 & +0.02 & 0.06 \\
\hline Eu II & 4129.72 & -0.02 & 0.07 & -0.02 & 0.07 & -0.02 & 0.07 & -0.02 & 0.07 \\
\hline Eu II & 4205.04 & -0.02 & 0.04 & -0.02 & 0.04 & -0.02 & 0.04 & -0.02 & 0.04 \\
\hline Gd II & 4049.85 & +0.02 & 0.08 & +0.02 & 0.08 & +0.02 & 0.08 & +0.02 & 0.08 \\
\hline Gd II & 4130.37 & -0.10 & 0.08 & -0.10 & 0.08 & -0.10 & 0.08 & -0.10 & 0.08 \\
\hline Gd II & 4251.73 & +0.09 & 0.08 & +0.09 & 0.08 & +0.09 & 0.08 & +0.09 & 0.08 \\
\hline Dy II & 3757.37 & +0.05 & 0.20 & +0.05 & 0.20 & +0.05 & 0.20 & +0.05 & 0.20 \\
\hline Dy II & 3944.68 & +0.07 & 0.20 & +0.07 & 0.20 & +0.07 & 0.20 & +0.07 & 0.20 \\
\hline Dy II & 4103.31 & -0.15 & 0.20 & -0.15 & 0.20 & -0.15 & 0.20 & -0.15 & 0.20 \\
\hline Dy II & 4449.70 & +0.04 & 0.20 & +0.04 & 0.20 & +0.04 & 0.20 & +0.04 & 0.20 \\
\hline Er II & 3729.52 & -0.01 & 0.09 & -0.01 & 0.09 & -0.01 & 0.09 & -0.01 & 0.09 \\
\hline Er II & 3830.48 & +0.02 & 0.03 & +0.02 & 0.03 & +0.02 & 0.03 & +0.02 & 0.03 \\
\hline Er II & 3896.23 & -0.02 & 0.06 & -0.02 & 0.06 & -0.02 & 0.06 & -0.02 & 0.06 \\
\hline
\end{tabular}

Bonifacio et al. (2009) sample of dwarfs. We perform a straight comparison of the results without accounting for differences in, e.g., the set of lines used or the transition probabilities.

The results of this comparison are listed in Table 17. The $[\mathrm{X} / \mathrm{Fe}]$ ratios, many derived from transitions in neutral atoms, are generally overabundant in our analysis. This can easily be traced to the fact that our iron abundances derived from $\mathrm{Fe}_{\mathrm{I}}$ lines are $\sim 0.1$ dex lower than the iron abundance derived from $\mathrm{Fe}$ II (Figure 14), which are themselves lower than published $[\mathrm{Fe} / \mathrm{H}]$ values by $0.27 \mathrm{dex}$, on average, for red giants (Table 9). A lower $[\mathrm{Fe} / \mathrm{H}]$ value increases $[\mathrm{X} / \mathrm{Fe}]$ if element $\mathrm{X}$ is not also derived from lines of similar excitation potential and strength. Ratios of neutral elements with the largest positive discrepancies, $[\mathrm{Mg} / \mathrm{Fe}]$ and $[\mathrm{Si} / \mathrm{Fe}]$, are often derived from (only) strong $\mathrm{Mg}$ I and $\mathrm{Si}$ I lines that are sensitive to the microturbulent velocity, and our derived $v_{\mathrm{t}}$ values are lower than those derived by previous studies (Table 9 ). This drives the $[\mathrm{Mg} / \mathrm{Fe}]$ and $[\mathrm{Si} / \mathrm{Fe}]$ ratios to even higher values. Our line-by-line corrections (Table 16) further increase $[\mathrm{Si} / \mathrm{Fe}]$ when the $3905 \AA \mathrm{Si}$ I line is considered. The underabundances of $[\mathrm{Sc} / \mathrm{Fe}]$ and $[\mathrm{Co} / \mathrm{Fe}]$ with respect to Cayrel et al. (2004) can be accounted for by our inclusion of the hyperfine structure for Co I and Sc II lines, which desaturate the lines and lower the derived abundances. In principle, additional discrepancies may arise from differences in the transition probabilities, although we have not checked this explicitly since doing so would require examining which sets of lines of each species were employed in each star by each analysis.

\subsubsection{Dispersion in the $[X / F e]$ Ratios}

We can also compare the standard deviation of the $[\mathrm{X} / \mathrm{Fe}]$ ratios for stars in common with the First Stars, McWilliam et al. (1995a), and Yong et al. (2013) samples. In the absence of cosmic dispersion, this comparison offers an independent quantitative measure of the precision of the derived abundances. In practice, many of these abundance ratios do exhibit at least a small amount of cosmic dispersion, so the standard deviations are useful in a comparative sense. Table 18 lists the standard deviation in each ratio and the number of stars used to compute it for each of the four surveys.

The First Stars survey used higher quality spectroscopic observations than our survey or that of McWilliam et al. (1995b). The typical spectral resolution and $\mathrm{S} / \mathrm{N}$ ratios are also listed in Table 18 for the stars in common among these three surveys. The First Stars observations correspond to an increase of a factor of $\approx 10$ in total photons $\AA^{-1}$ over our data and a factor of $>70$ over the data of McWilliam et al. 
Table 17

Comparison of $[\mathrm{X} / \mathrm{Fe}]$ Ratios with Previous Studies

\begin{tabular}{|c|c|c|c|c|}
\hline Ratio & McWilliam et al. (1995a) ${ }^{\mathrm{a}}$ & Cayrel et al. (2004) ${ }^{\mathrm{b}}$ & Yong et al. (2013) & Bonifacio et al. (2009) \\
\hline$[\mathrm{C} / \mathrm{Fe}]$ & $-0.05(0.18,12)$ & $-0.14(0.20,17)$ & $\ldots$ & $+0.22(0.15,5)$ \\
\hline$[\mathrm{N} / \mathrm{Fe}]$ & $\ldots$ & $-0.12(0.23,13)$ & $\ldots$ & $\ldots$ \\
\hline$[\mathrm{Na} / \mathrm{Fe}]$ & $+0.15(0.13,5)$ & $+0.08(0.19,6)$ & $-0.07(0.21,6)$ & $\ldots$ \\
\hline$[\mathrm{Mg} / \mathrm{Fe}]$ & $+0.23(0.18,14)$ & $+0.26(0.14,18)$ & $+0.28(0.13,18)$ & $+0.17(0.08,9)$ \\
\hline$[\mathrm{Al} / \mathrm{Fe}]$ & $+0.01(0.25,14)$ & $+0.16(0.14,18)$ & $+0.08(0.15,18)$ & $\ldots$ \\
\hline [Si/Fe] & $+0.33(0.29,11)$ & $+0.48(0.20,15)$ & $\ldots$ & $+0.26(0.12,9)$ \\
\hline$[\mathrm{Ca} / \mathrm{Fe}]$ & $+0.04(0.10,14)$ & $+0.15(0.09,18)$ & $+0.16(0.07,18)$ & $+0.14(0.05,9)$ \\
\hline$[\mathrm{Sc} / \mathrm{Fe}]$ & $-0.28(0.25,14)$ & $-0.19(0.08,18)$ & $-0.30(0.11,18)$ & $-0.25(0.20,9)$ \\
\hline [Ti I/Fe] & $-0.11(0.21,12)$ & $-0.05(0.07,18)$ & $-0.10(0.08,18)$ & \\
\hline$[\mathrm{Ti}$ II $/ \mathrm{Fe}]$ & $-0.16(0.13,14)$ & $-0.07(0.10,18)$ & $-0.11(0.18,18)$ & $-0.23(0.18,9)$ \\
\hline$[\mathrm{CrI} / \mathrm{Fe}]$ & $+0.12(0.15,14)$ & $+0.11(0.08,18)$ & $+0.11(0.10,18)$ & $+0.01(0.10,9)$ \\
\hline$[\mathrm{Mn} \mathrm{I} / \mathrm{Fe}]$ & $+0.22(0.30,13)$ & $-0.08(0.12,18)$ & $+0.05(0.21,8)$ & $+0.22(0.11,8)$ \\
\hline$[\mathrm{Fe} \mathrm{I} / \mathrm{H}]$ & $-0.41(0.14,14)$ & $-0.31(0.12,18)$ & $-0.31(0.13,18)$ & $-0.27(0.15,9)$ \\
\hline$[\mathrm{Fe}$ II $/ \mathrm{H}]$ & $-0.27(0.14,14)$ & $-0.20(0.13,18)$ & $-0.21(0.13,18)$ & $-0.10(0.13,9)$ \\
\hline$[\mathrm{Co} / \mathrm{Fe}]$ & $-0.28(0.11,14)$ & $-0.29(0.13,18)$ & $-0.16(0.09,18)$ & $-0.15(0.12,7)$ \\
\hline$[\mathrm{Ni} / \mathrm{Fe}]$ & $-0.23(0.31,13)$ & $+0.12(0.10,18)$ & $+0.07(0.11,18)$ & $+0.05(0.11,9)$ \\
\hline$[\mathrm{Zn} / \mathrm{Fe}]$ & $\ldots$ & $+0.21(0.12,16)$ & $\ldots$ & $\ldots$ \\
\hline$[\mathrm{Sr} / \mathrm{Fe}]$ & $-0.06(0.25,14)$ & $-0.03(0.20,17)$ & $+0.07(0.45,7)$ & $-0.32(0.45,9)$ \\
\hline$[\mathrm{Ba} / \mathrm{Fe}]$ & $+0.04(0.25,11)$ & $-0.24(0.18,16)$ & $\ldots$ & $+0.01(0.52,3)$ \\
\hline
\end{tabular}

Notes. Differences are in the sense of this study minus the other. Each entry represents the mean difference, standard deviation, and number of stars: $\langle\Delta\rangle(\sigma, N)$. ${ }^{a}$ Includes [Ba/Fe] from McWilliam (1998).

${ }^{b}$ Includes $[\mathrm{C} / \mathrm{Fe}]$ and $[\mathrm{N} / \mathrm{Fe}]$ from Spite et al. (2005) and $[\mathrm{Sr} / \mathrm{Fe}]$ and $[\mathrm{Ba} / \mathrm{Fe}]$ from François et al. (2007).

Table 18

Data Quality and Dispersions in Abundance Ratios for 18 Stars in Common among Four Large Surveys

\begin{tabular}{lcccc}
\hline \hline & This Study & First Stars & Yong et al. (2013) & McWilliam et al. (1995b, 1995a) \\
\hline Resolution $\equiv \lambda / \Delta \lambda$ & 41000 & 47000 & $\ldots$ & 22000 \\
$\lambda$ of S/N estimate $^{-1}$ & $3950 \AA$ & $4000 \AA$ & $\ldots$ \\
Median S/N pixel $^{-1}$ & 72 & 150 & $\ldots$ & 35 \\
Median S/N RE $^{-1}$ & 112 & 335 & $\ldots$ & 67 \\
${\text { Median photons }{ }^{-1}}$ & $1.3 \times 10^{5}$ & $1.3 \times 10^{6}$ & $\ldots$ & $\sigma .0 \times 10^{4}$ \\
\hline$[\mathrm{C} / \mathrm{Fe}]$ & $\sigma(N)$ & $\sigma(N)$ & $\sigma(N)$ & $0.48(12)$ \\
{$[\mathrm{N} / \mathrm{Fe}]$} & $0.54(17)$ & $0.49(17)$ & $\ldots$ & $\ldots$ \\
{$[\mathrm{Na} / \mathrm{Fe}]$} & $0.78(12)$ & $0.70(15)$ & $\ldots$ & $0.64(13)$ \\
{$[\mathrm{Mg} / \mathrm{Fe}]$} & $0.15(6)$ & $0.44(14)$ & $0.55(15)$ & $0.31(14)$ \\
{$[\mathrm{Al} / \mathrm{Fe}]$} & $0.28(18)$ & $0.33(18)$ & $0.31(18)$ & $0.38(14)$ \\
{$[\mathrm{Si} / \mathrm{Fe}]$} & $0.27(18)$ & $0.21(18)$ & $\ldots$ & $0.28(14)$ \\
{$[\mathrm{Ca} / \mathrm{Fe}]$} & $0.25(15)$ & $0.17(18)$ & $0.12(18)$ & $0.18(14)$ \\
{$[\mathrm{Sc} / \mathrm{Fe}]$} & $0.13(18)$ & $0.12(18)$ & $0.16(18)$ & $0.26(14)$ \\
{$[\mathrm{Ti} / \mathrm{Fe}]$} & $0.12(18)$ & $0.12(18)$ & $0.14(18)$ & $0.26(12)$ \\
{$[\mathrm{Ti} / \mathrm{II} / \mathrm{Fe}]$} & $0.17(18)$ & $0.11(18)$ & $0.21(18)$ & $0.14(14)$ \\
{$[\mathrm{Cr} / \mathrm{Fe}]$} & $0.15(18)$ & $0.13(18)$ & $0.06(18)$ & $0.17(14)$ \\
{$[\mathrm{Mn} / \mathrm{Fe}]$} & $0.08(18)$ & $0.06(18)$ & $0.24(8)$ & $0.24(13)$ \\
{$[\mathrm{Co} / \mathrm{Fe}]$} & $0.20(18)$ & $0.13(18)$ & $0.21(18)$ & $0.19(14)$ \\
{$[\mathrm{Ni} / \mathrm{Fe}]$} & $0.21(18)$ & $0.16(18)$ & $0.15(18)$ & $\ldots$ \\
{$[\mathrm{Zn} / \mathrm{Fe}]$} & $0.13(18)$ & $0.14(18)$ & $\ldots$ & $0.34(13)$ \\
{$[\mathrm{Sr} / \mathrm{Fe}]$} & $0.24(16)$ & $0.19(18)$ & $\ldots .47(7)$ & $0.80(14)$ \\
{$[\mathrm{Ba} / \mathrm{Fe}]^{a}$} & $0.83(18)$ & $0.72(17)$ & $\ldots .71(11)$ \\
\hline
\end{tabular}

Note. ${ }^{a}$ Includes $[\mathrm{Ba} / \mathrm{Fe}]$ from McWilliam (1998).

Figure 20 illustrates these results for the $[\mathrm{Mg} / \mathrm{Fe}]$ through $[\mathrm{Zn} / \mathrm{Fe}]$ ratios, which are measured in most stars in each of these four surveys. The naive expectation would be that the abundance precision should roughly correlate with the data quality, and the dispersions generally support this expectation. The dispersions are generally smallest for the First Stars sample, followed by the Yong et al. (2013) rederivation and our sample, followed by the McWilliam et al. (1995a) sample. We do not achieve the same internal precision as the First Stars survey does for the giants; however, we have achieved reasonable precision on a photon budget roughly 10 times lower per star.

\subsubsection{Detailed Comparisons of [X/Fe] for Individual Stars}

We also compare the detailed abundance patterns of two wellstudied stars. CS 22892-052 is a well-studied giant with a high level of $r$-process enhancement. CS 22949-037 is another wellstudied giant with substantial enhancement of carbon, nitrogen, and other light elements. The abundance pattern of each of these 


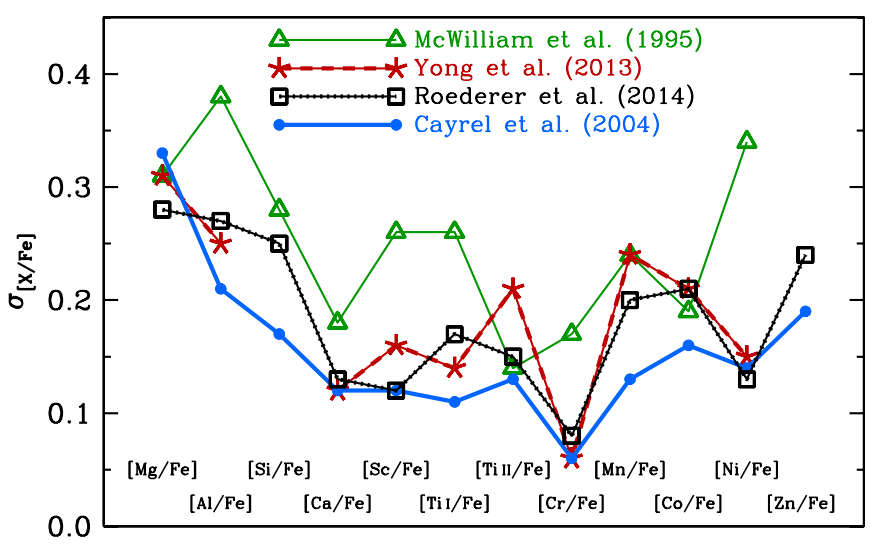

Abundance Ratio

Figure 20. Dispersion in the derived abundance ratios of stars in common between our survey (Roederer et al. 2014), McWilliam et al. (1995a), Yong et al. (2013), and Cayrel et al. (2004). The number of stars used to compute each dispersion is listed in Table 18.

(A color version of this figure is available in the online journal.)

stars has been subject to close scrutiny by investigators over the last two decades, and we limit our comparison to a few extensive studies of each. Figure 21 illustrates these comparisons, and references are given in the figure caption.

In general the agreement is superb, but a couple of features stand out. Our $[\mathrm{Si} / \mathrm{Fe}]$ ratios are higher than found by previous work, as discussed above. The other prominent difference is that the $[\mathrm{X} / \mathrm{Fe}]$ ratios, where $\mathrm{X}$ represents one of the neutroncapture elements detected in its ionized state, are lower in CS 22892-052 when compared with previous results (Sneden et al. 1994, 1996, 2003, 2009; McWilliam et al. 1995a; Honda et al. 2004b; Barklem et al. 2005; François et al. 2007). For example, our derived $[\mathrm{Eu} / \mathrm{Fe}]$ ratios are lower by $0.20 \operatorname{dex}(\sigma=0.07)$ on average. Figure 21 illustrates that our derived ratios among the neutron-capture elements in CS 22892-052 are in very good agreement with the previous studies. In other words, there is generally a constant offset between our $[\mathrm{X} / \mathrm{Fe}]$ ratios and those of Sneden et al. and François et al. (The $[\mathrm{X} / \mathrm{Fe}]$ ratios derived by François et al. are also systematically lower than those derived by Sneden et al. by about half as much as ours.) Our results for CS 31082-001, another giant with a high level of $r$-process enrichment, exhibit a similar offset with respect to Hill et al. (2002), Sneden et al. (2009), and others. Our derived abundance ratios for well-studied giants with sub-solar [Eu/Fe] ratios, like HD 122563 and HD 128279, are similarly low (cf., e.g., Johnson 2002).

The explanation for this offset is the cumulative effect of several differences between our study and previous ones, and we demonstrate this using the Sneden et al. (2003) analysis of CS 22892-052. For this test, we adopt the model preferred by Sneden et al., their Fe II EW measurements, their $\log g f$ values, their solar abundances, and we only consider the four Eu II lines in common with our study. Using the appropriate earlier version of MOOG, we derive $[\mathrm{Fe} / \mathrm{H}]=-3.09$ from $\mathrm{Fe}$ II lines (identical to Sneden et al.) and $[\mathrm{Eu} / \mathrm{Fe}]=+1.57$ from our spectrum. This is 0.22 dex higher than our result, and it is in much better agreement with the value derived by Sneden et al., +1.64 . The remaining difference must be attributed to their higher-quality spectrum of CS 22892-052.

In summary, the abundance differences can be attributed to different, but reasonable, choices made during the course of the each analysis.

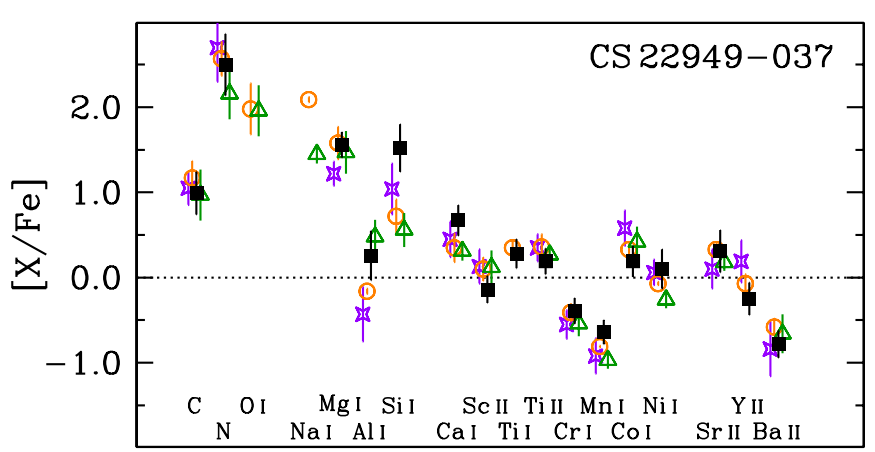

Species
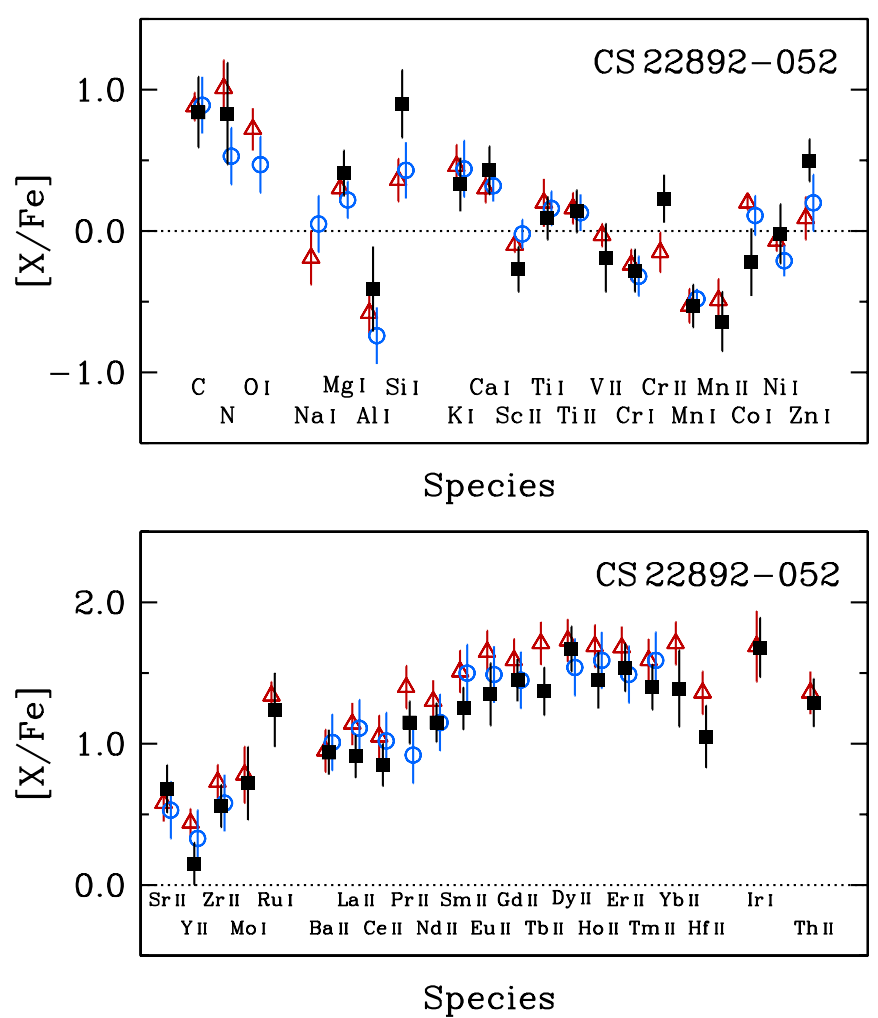

Figure 21. Comparison of our derived $[\mathrm{X} / \mathrm{Fe}]$ ratios with previous work for two well-studied stars, CS 22949-037 and CS 22892-052. In the top panel, our results (black squares) are compared with those of Norris et al. (2001; purple stars), Depagne et al. (2002; orange circles), and Cohen et al. (2008; green triangles). In the middle and bottom panels, our results are compared with those of Sneden et al. (2003, 2009) and Cowan et al. (2005; red triangles) as well as Cayrel et al. (2004) and François et al. (2007; blue circles).

(A color version of this figure is available in the online journal.)

\subsection{Abundance Trends with Effective Temperature}

Figures 22 through 51 illustrate the relationship between the derived abundances and $T_{\text {eff }}$ for most species examined. Each of these figures is subdivided into four panels, one each for the four classes of stars in our study. Similar comparisons for Ga I, Rb I, $\mathrm{Nb}$ II, Mo I, Tc I, Ru I, Sn I, Ir I, and Pb I are generally comprised of uninteresting upper limits. Similar comparisons for most of the rare earth elements, Hf II, and Th II closely resemble the La II and Eu II abundances shown in Figures 49 and 50.

Many of these species show no [X/Fe] trends with $T_{\text {eff }}$ (where $\mathrm{X}$ stands for the element of interest). The $[\mathrm{Ca} / \mathrm{Fe}]$ ratios shown in Figure 31, for example, demonstrate this scenario. Other species show a false trend in that lines of species of low abundance can only be detected in the coolest stars. The $[\mathrm{Eu} / \mathrm{Fe}]$ ratios shown in 


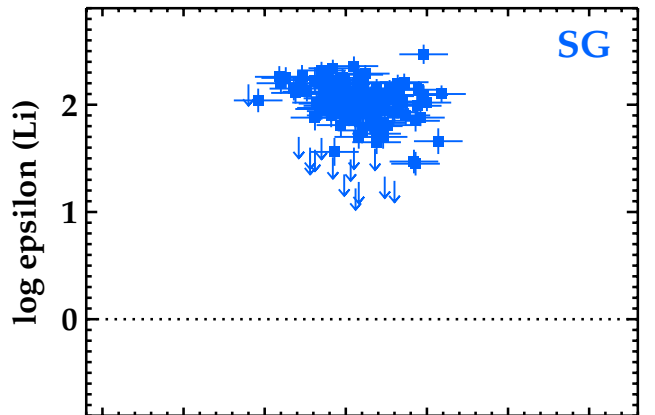

7500700065006000550050004500 $\mathrm{T}_{\text {eff }}(\mathrm{K})$

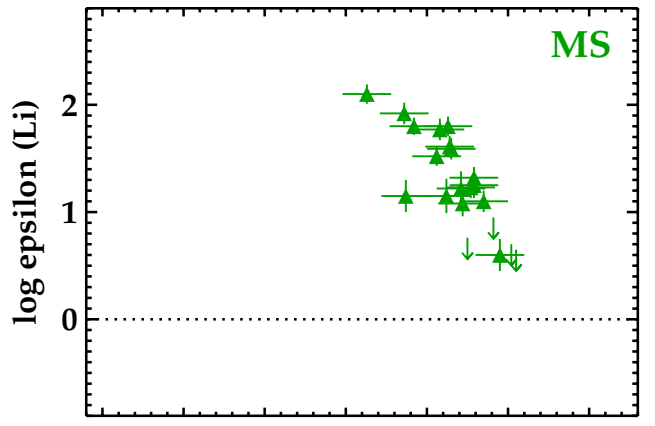

7500700065006000550050004500

$\mathrm{T}_{\text {eff }}(\mathrm{K})$

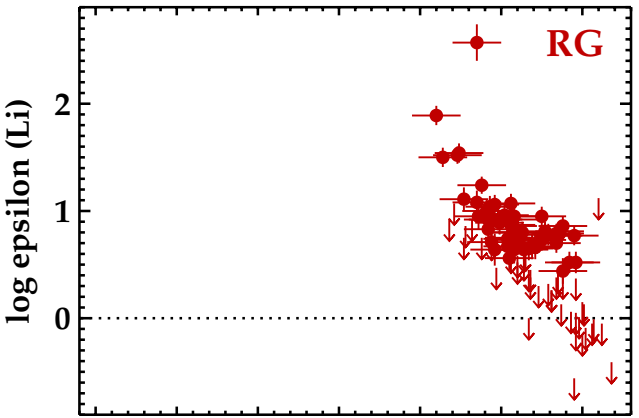

7500700065006000550050004500 $T_{\text {eff }}(K)$

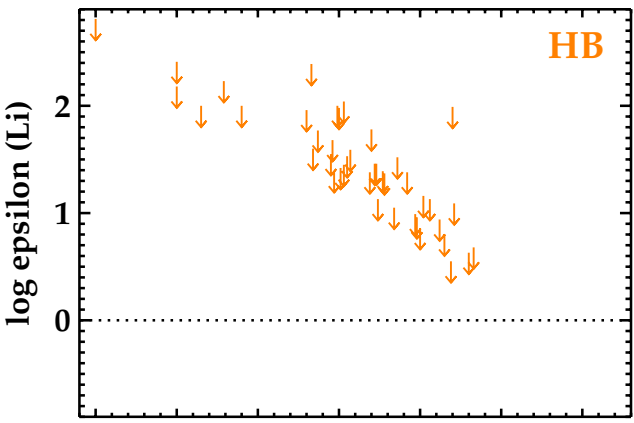

7500700065006000550050004500

$$
\mathrm{T}_{\text {eff }}(\mathrm{K})
$$

Figure 22. Derived lithium abundances as a function of $T_{\text {eff }}$. Each panel illustrates a different class of stars, and the three stars in class "BS" are included in the plot for stars in class "HB." The $1 \sigma$ uncertainties are indicated. Downward arrows indicate $3 \sigma$ upper limits, and the actual value is marked by the end with no arrow head. (A color version of this figure is available in the online journal.)

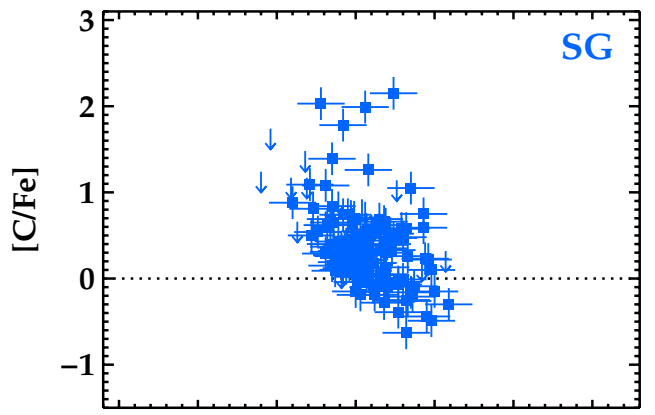

7500700065006000550050004500

$\mathrm{T}_{\text {eff }}(\mathrm{K})$

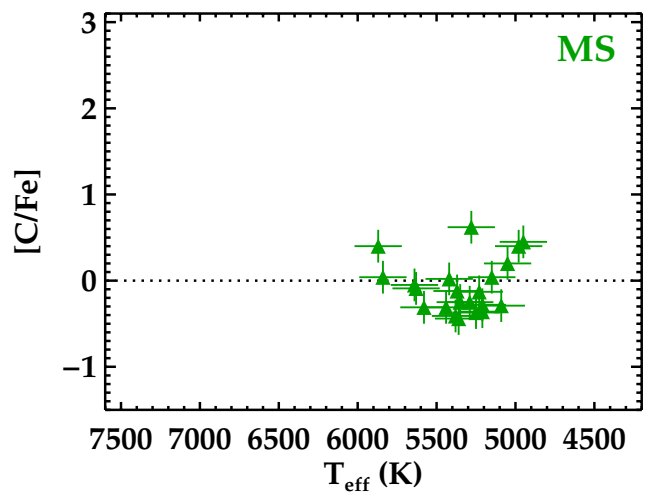

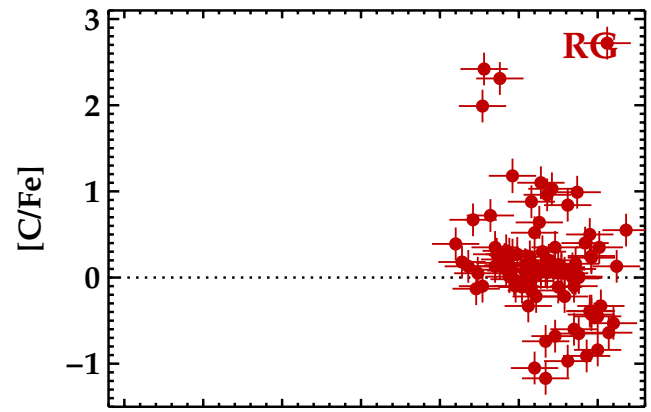

7500700065006000550050004500

$\mathrm{T}_{\text {eff }}(\mathrm{K})$

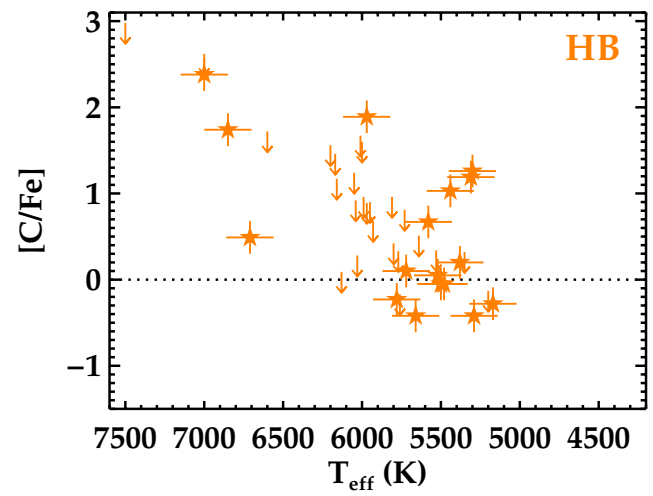

Figure 23. Derived $[\mathrm{C} / \mathrm{Fe}]$ ratios as a function of $T_{\text {eff }}$. The carbon abundance is derived from the $\mathrm{CH} A^{2} \Delta-X^{2} \Pi G$ band. The dotted lines indicate the solar ratio. All other symbols are the same as in Figure 22.

(A color version of this figure is available in the online journal.) 

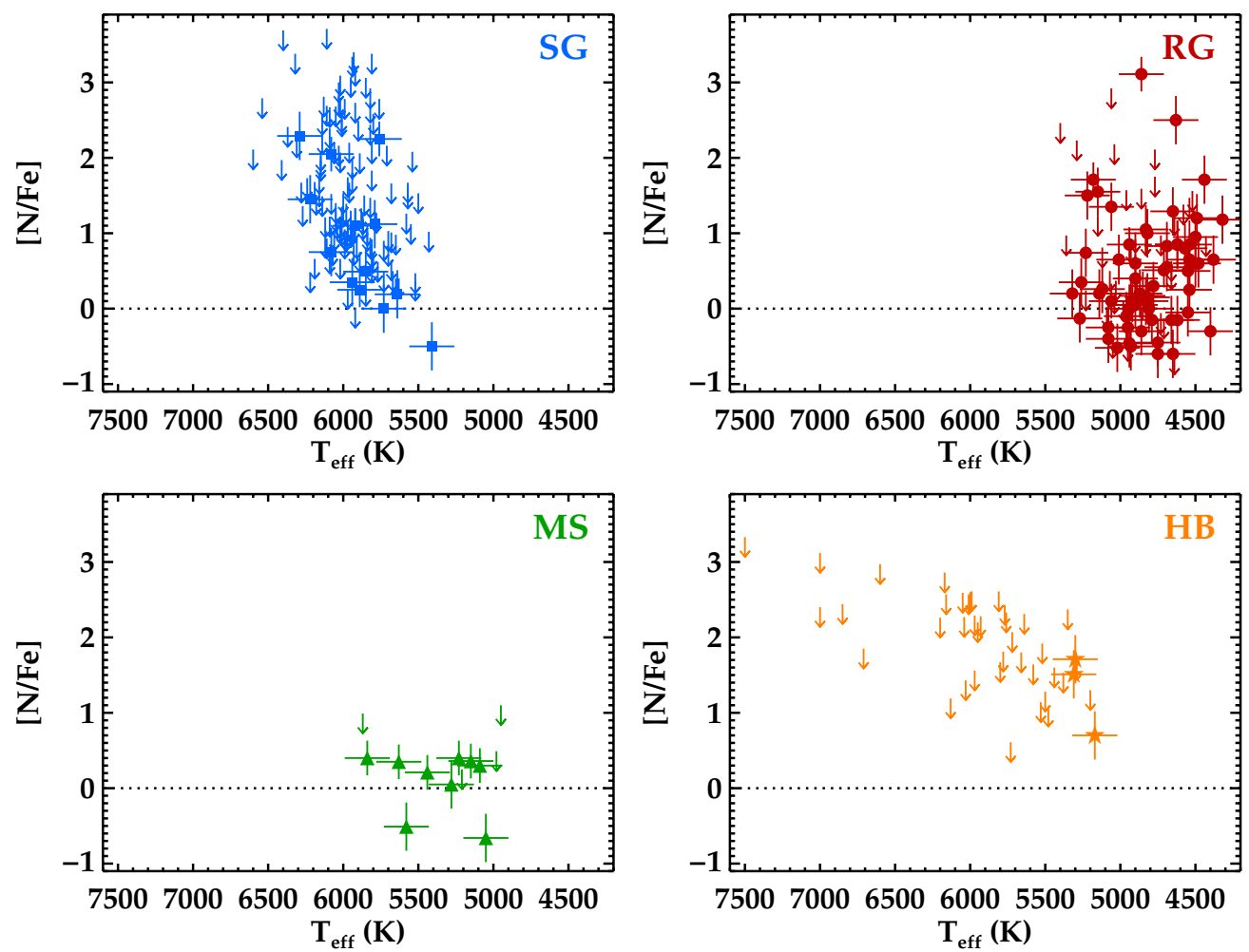

Figure 24. Derived $[\mathrm{N} / \mathrm{Fe}]$ ratios as a function of $T_{\text {eff. }}$. The nitrogen abundance is derived from the $\mathrm{NH} A^{3} \Pi-X^{3} \Sigma$ band or the CN $B^{2} \Sigma-X^{2} \Sigma$ band. Symbols are the same as in Figures 22 and 23.

(A color version of this figure is available in the online journal.)
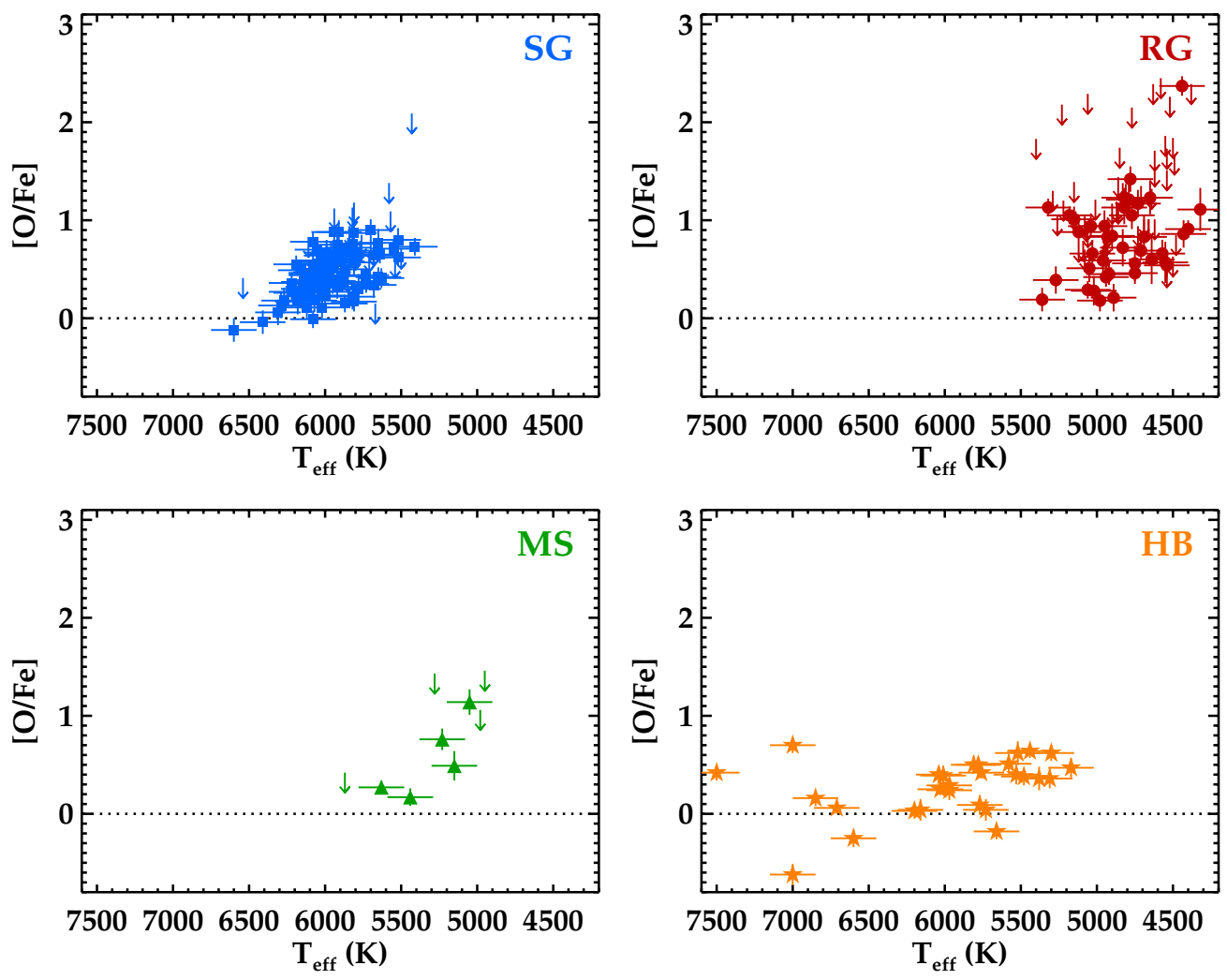

Figure 25. Derived $[\mathrm{O} / \mathrm{Fe}]$ ratios as a function of $T_{\text {eff. }}$. Symbols are the same as in Figures 22 and 23.

(A color version of this figure is available in the online journal.) 

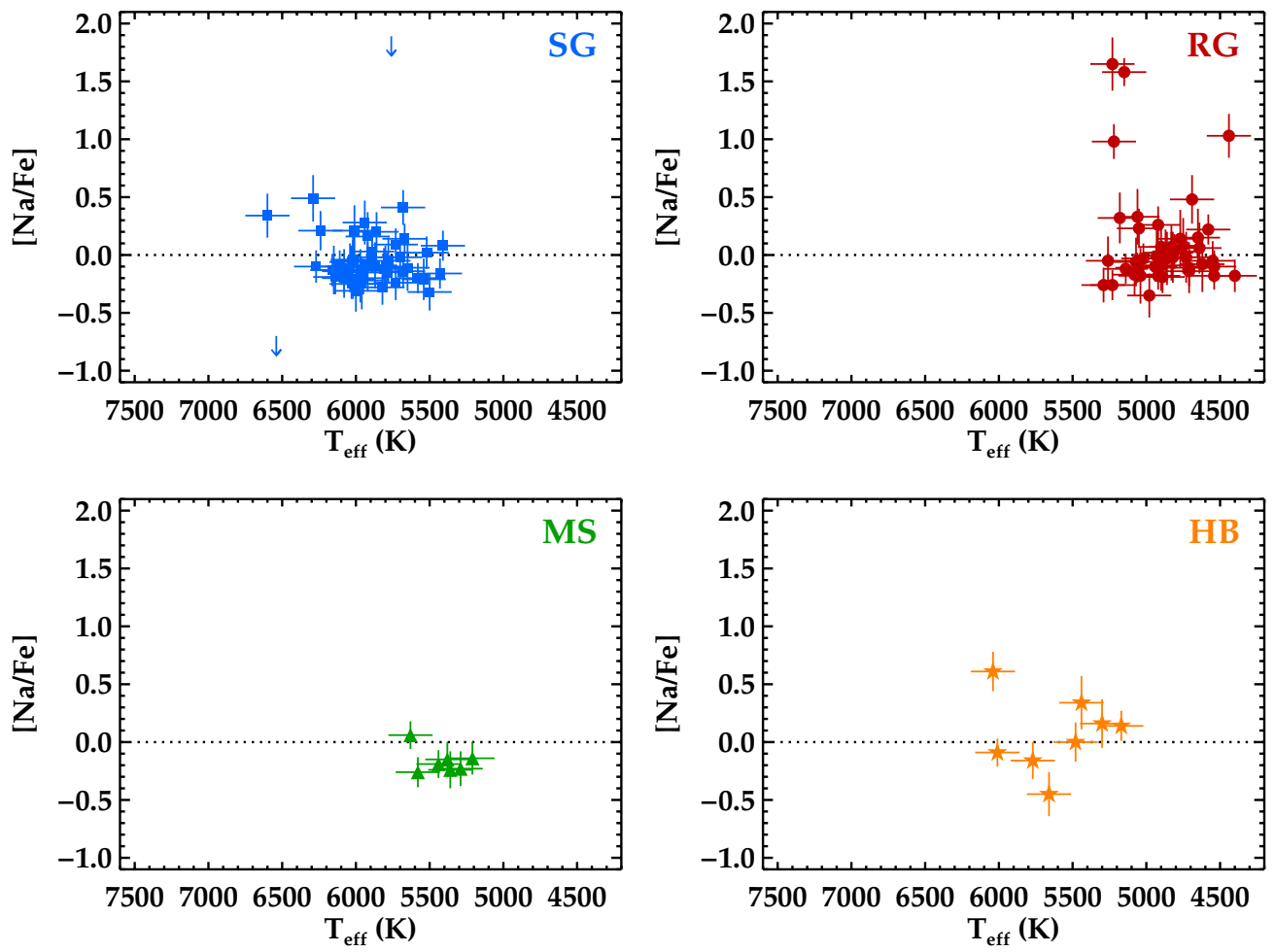

Figure 26. Derived $[\mathrm{Na} / \mathrm{Fe}]$ ratios as a function of $T_{\text {eff }}$. Symbols are the same as in Figures 22 and 23 .

(A color version of this figure is available in the online journal.)
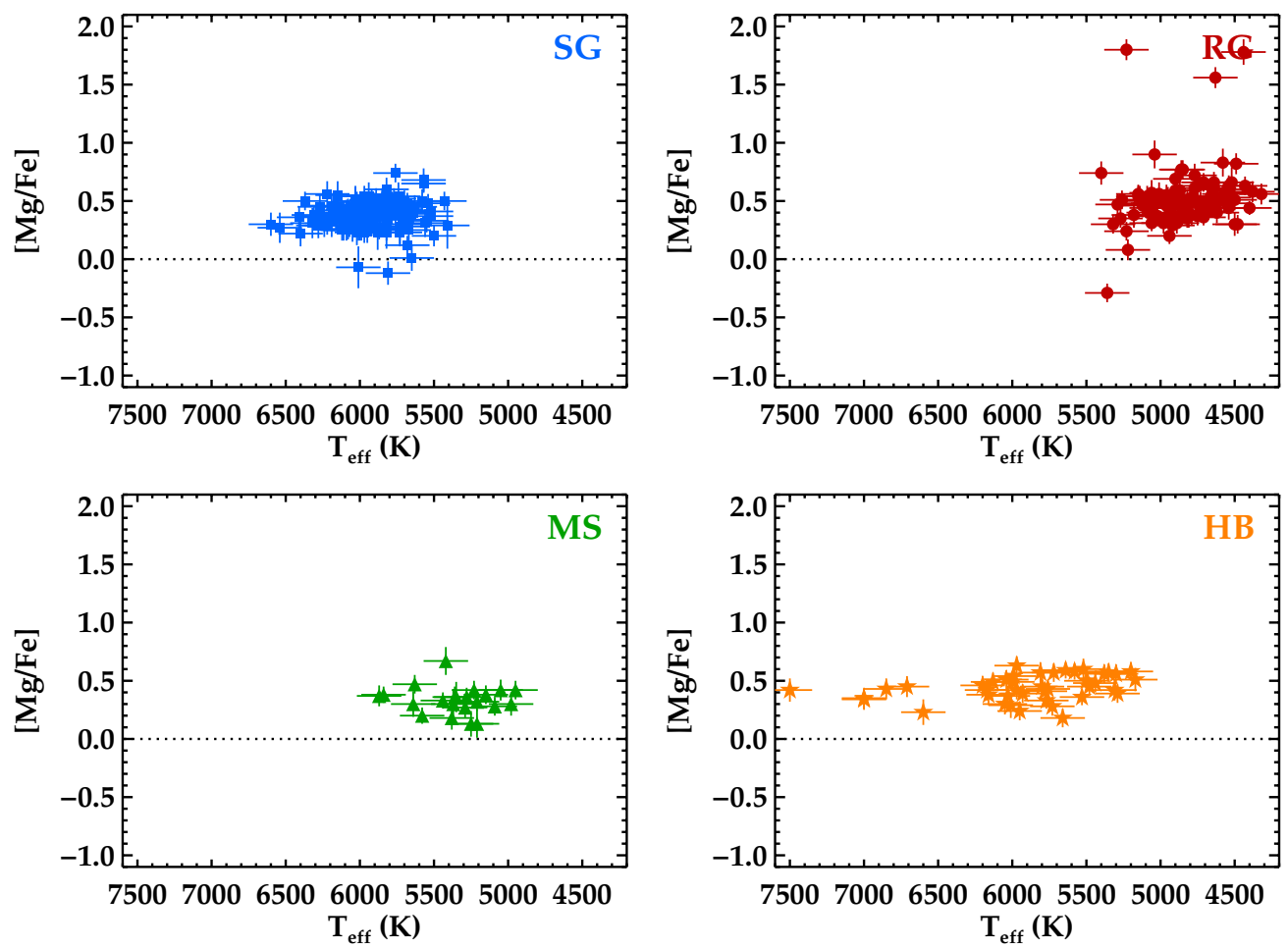

Figure 27. Derived $[\mathrm{Mg} / \mathrm{Fe}]$ ratios as a function of $T_{\text {eff }}$. Symbols are the same as in Figures 22 and 23.

(A color version of this figure is available in the online journal.)

Figure 50, for example, demonstrate this scenario. Other species show a real trend that relates to internal mixing during the course of normal stellar evolution. The lithium abundances and $[\mathrm{C} / \mathrm{Fe}]$ ratios shown in Figures 22 and 23, for example, demonstrate this scenario.
Other species show genuine abundance trends with $T_{\text {eff }}$, including Si I, Ti I, Cr I, Co I, and-to a lesser degree-O I, Sc II, V I, V II, Mn I, and Mn II. Several of these trends are apparent in stars along the subgiant and red giant branches as well as the $\mathrm{HB}$, suggesting it is not related to internal mixing or processing. 

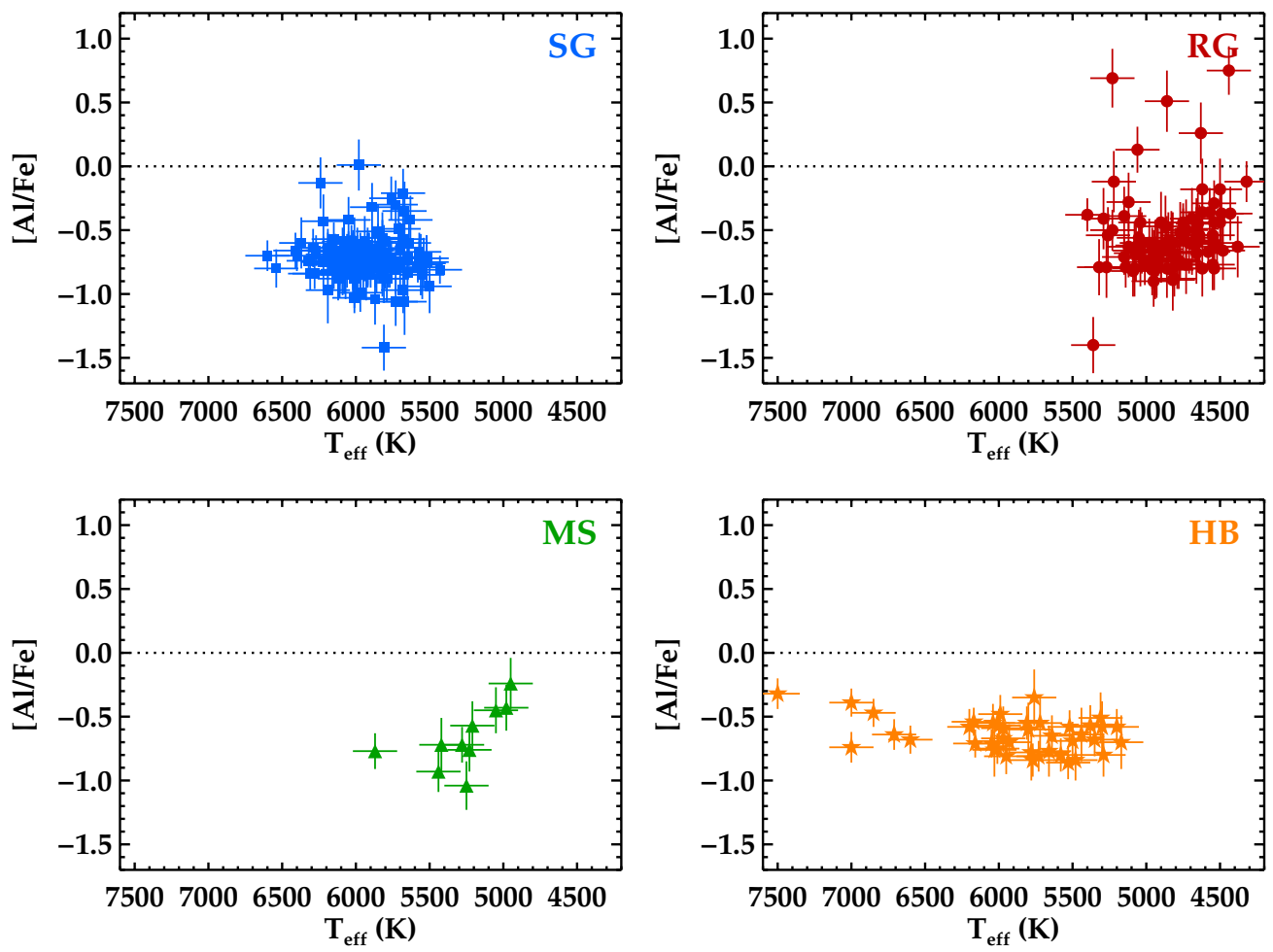

Figure 28. Derived $[\mathrm{Al} / \mathrm{Fe}]$ ratios as a function of $T_{\text {eff }}$. Symbols are the same as in Figures 22 and 23.

(A color version of this figure is available in the online journal.)
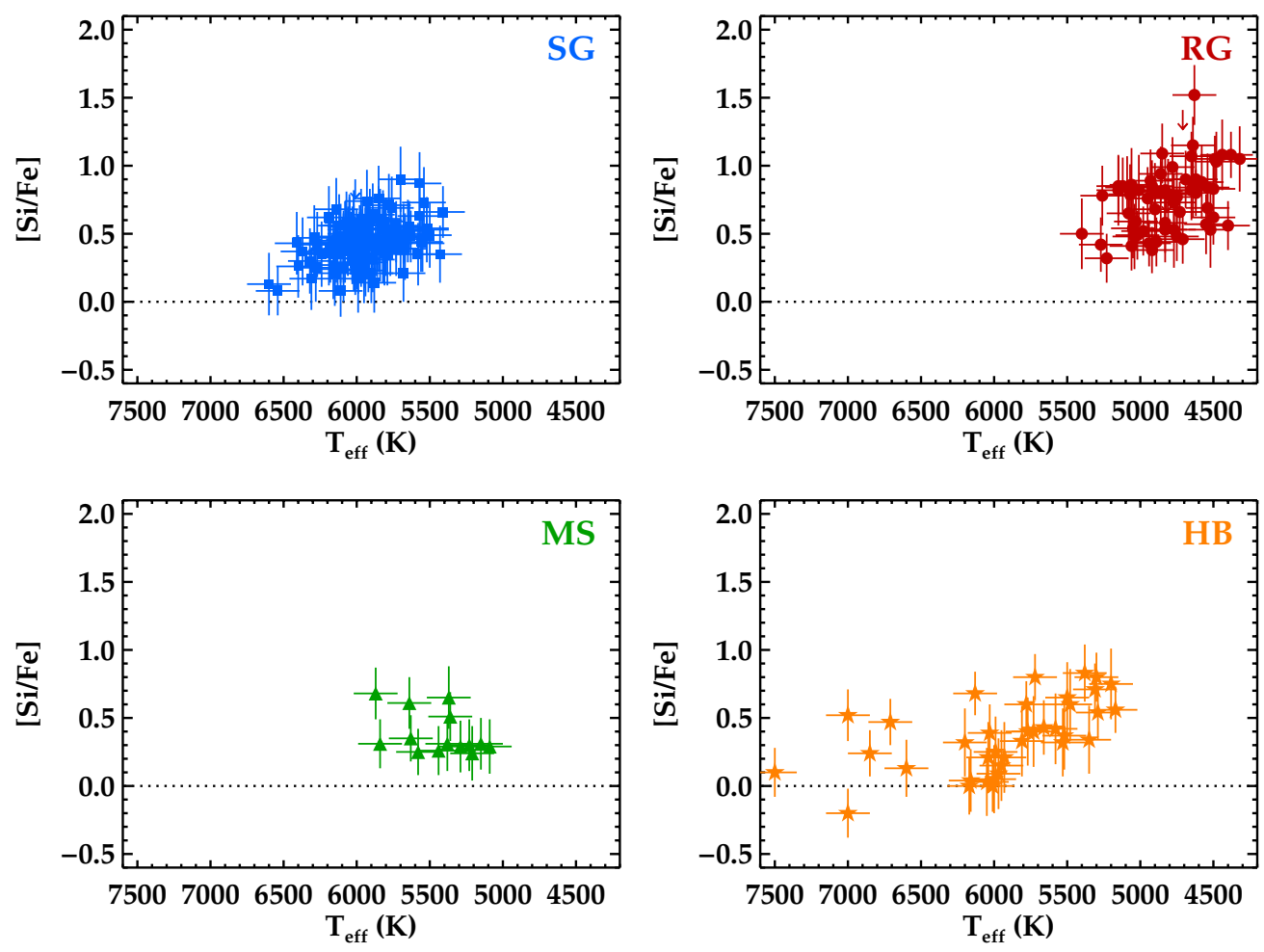

Figure 29. Derived [ $\mathrm{Si} / \mathrm{Fe}]$ ratios as a function of $T_{\text {eff }}$. Symbols are the same as in Figures 22 and 23.

(A color version of this figure is available in the online journal.)

Preston et al. (2006a) identified such an effect for Si I. Lai et al. (2008) also identified these trends in Si I, Ti I, and Cr I, although their study also found that $\mathrm{Ti}$ I and $\mathrm{Ti}$ II both show a trend with $T_{\text {eff }}$, while our Ti II abundances show no such trend.
The silicon abundance trend is characterized as showing higher abundances at lower temperatures. The silicon abundance is derived mostly from a single Si I line at $3905 \AA$, so an unidentified blend that grows stronger in cooler stars could, 

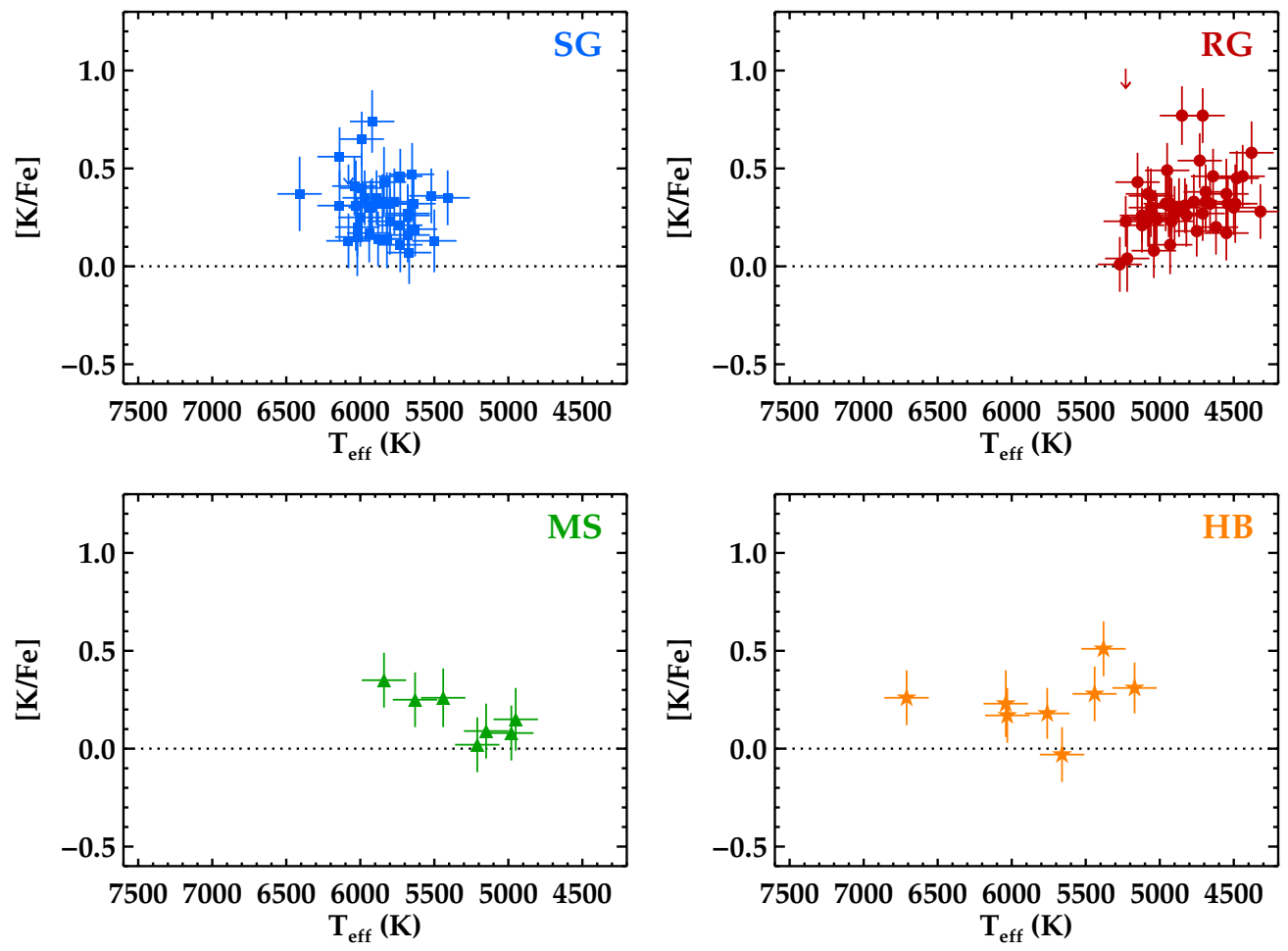

Figure 30. Derived $[\mathrm{K} / \mathrm{Fe}]$ ratios as a function of $T_{\text {eff }}$. Symbols are the same as in Figures 22 and 23.

(A color version of this figure is available in the online journal.)

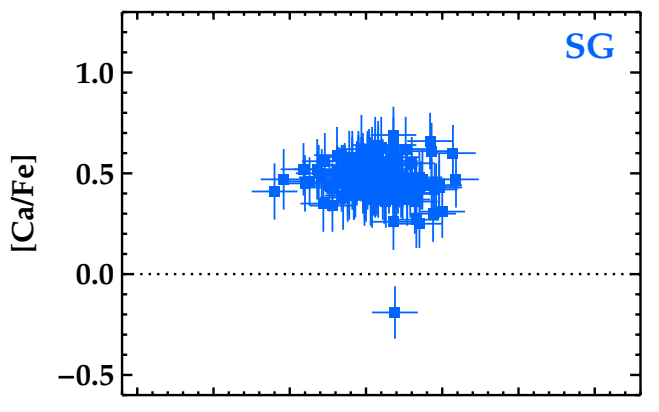

7500700065006000550050004500

$T_{\text {eff }}(\mathrm{K})$

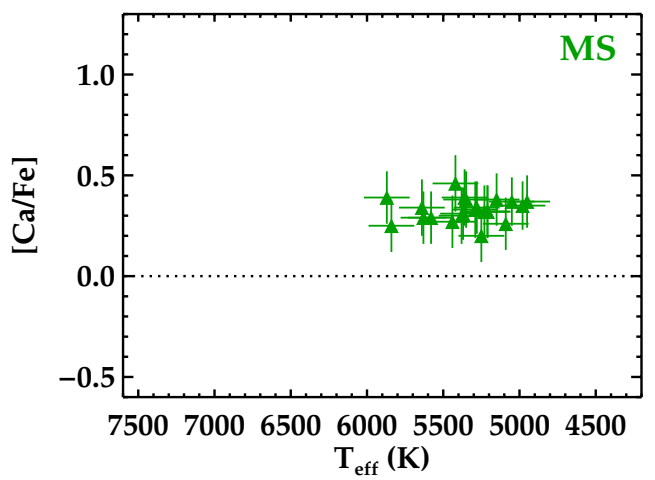

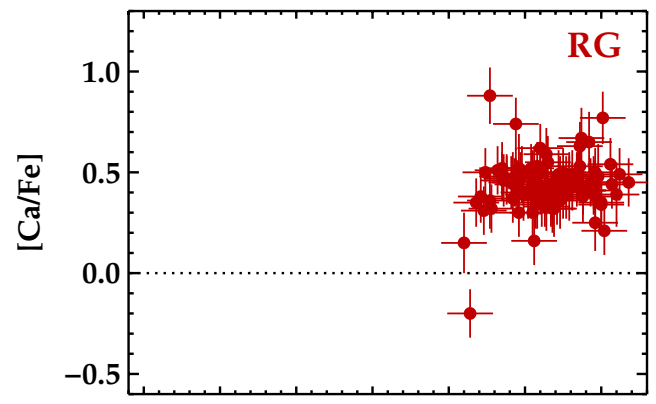

7500700065006000550050004500 $\mathrm{T}_{\text {eff }}(\mathrm{K})$

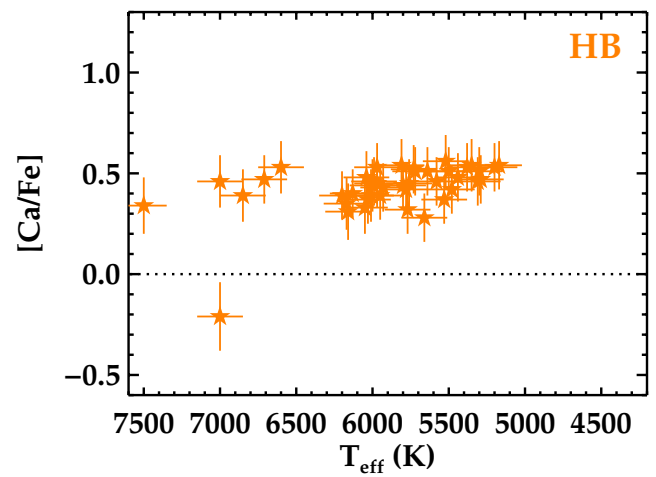

Figure 31. Derived $[\mathrm{Ca} / \mathrm{Fe}]$ ratios as a function of $T_{\text {eff }}$. Symbols are the same as in Figures 22 and 23.

(A color version of this figure is available in the online journal.)

in principle, explain this effect. Preston et al. (2006a) examined whether known $\mathrm{CH}$ molecular features could account for this extra absorption and concluded that this could bias the silicon abundance by a few percent at most, which is far insufficient to explain the observations. The non-LTE calculations of Shi et al.
(2009) suggest that the use of LTE could account for part of the discrepancy when using the Si I $3905 \AA$ line, but this matter is not fully resolved at present.

The oxygen trend with $T_{\text {eff }}$ goes in the same direction, but the oxygen abundance is usually derived from the O I triplet at 7771, 

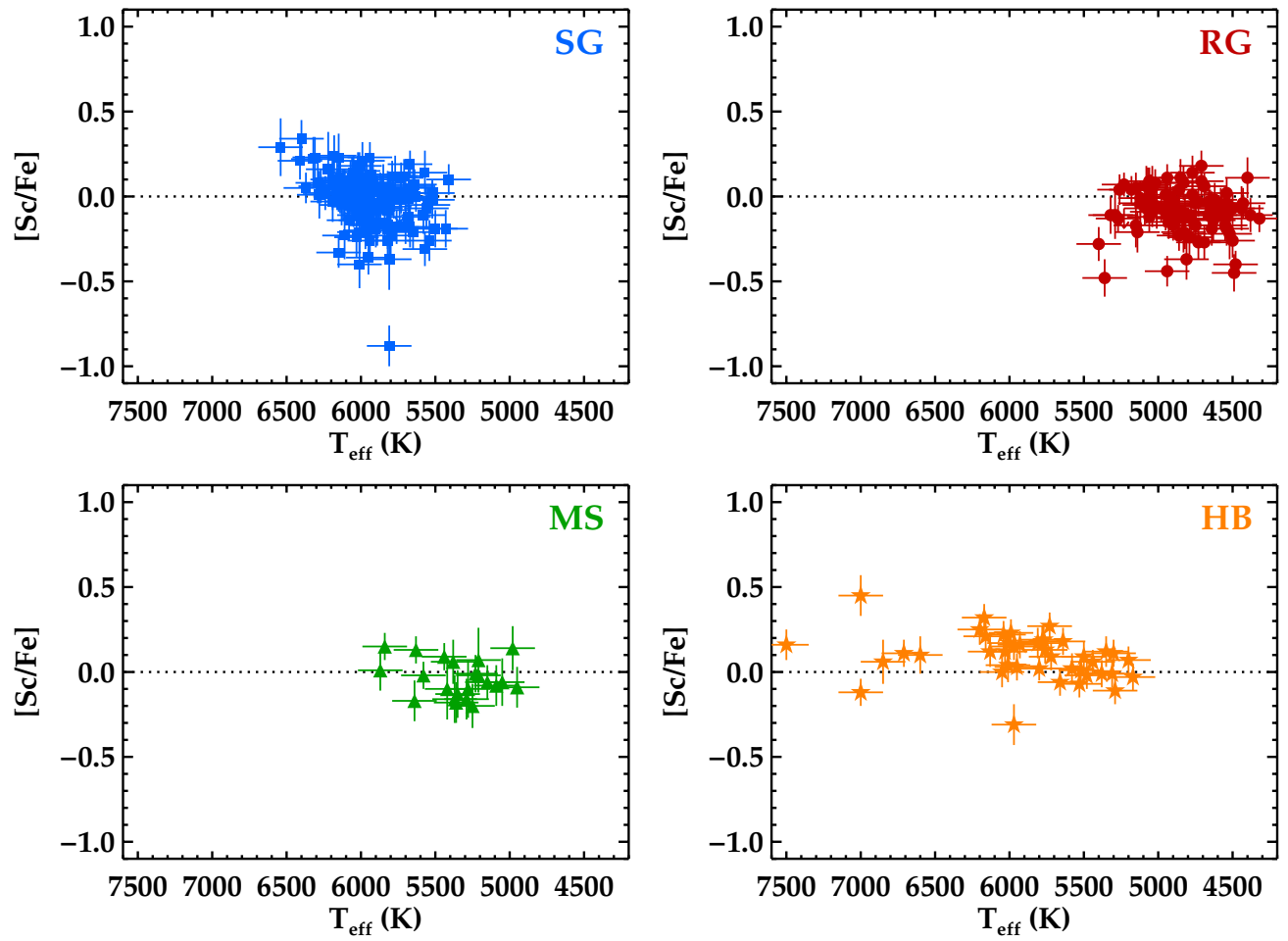

Figure 32. Derived [ $\mathrm{Sc} / \mathrm{Fe}]$ ratios as a function of $T_{\text {eff }}$. Symbols are the same as in Figures 22 and 23.

(A color version of this figure is available in the online journal.)
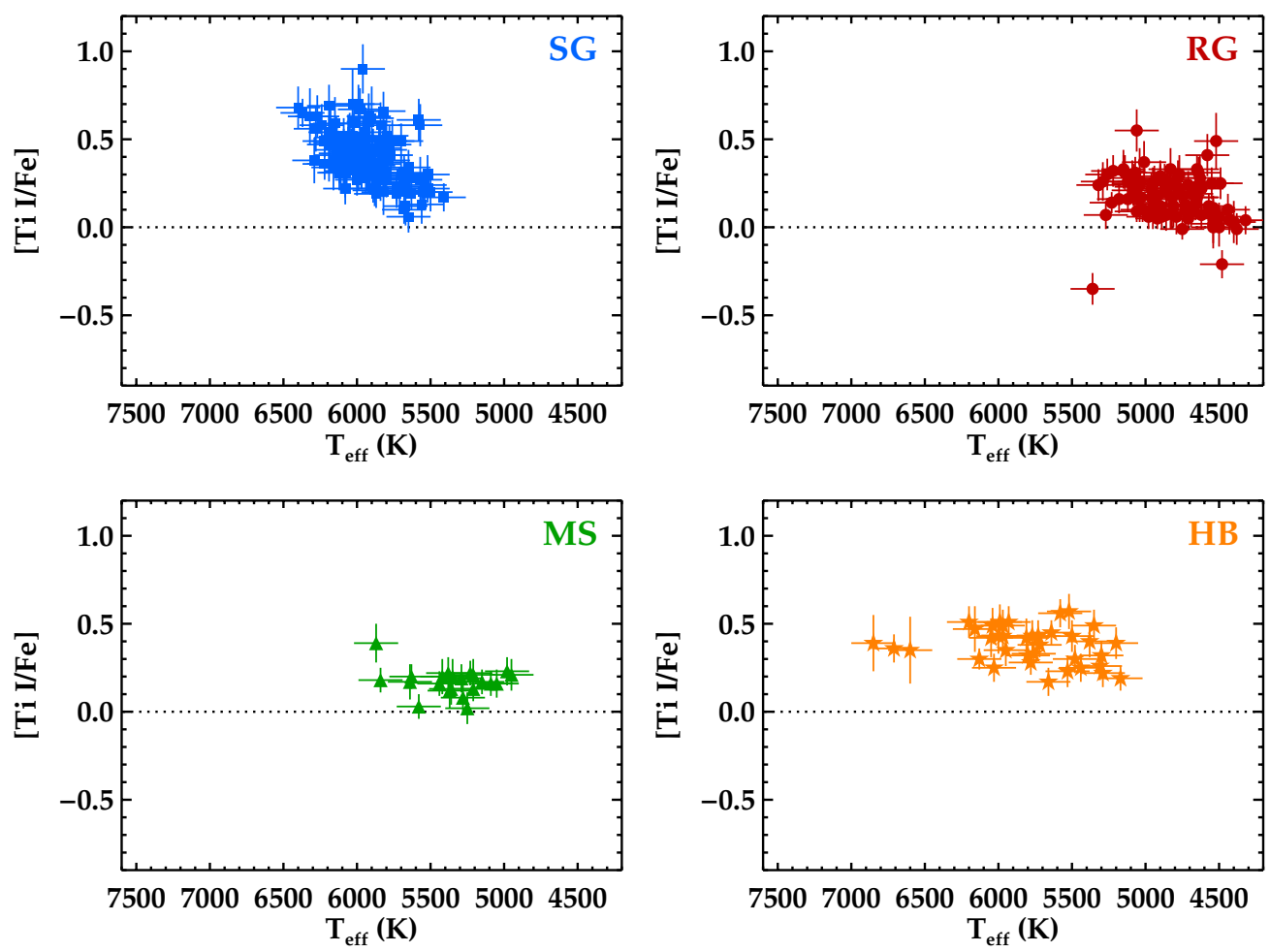

Figure 33. Derived $[\mathrm{Ti} / \mathrm{Fe}]$ ratios for neutral lines as a function of $T_{\mathrm{eff}}$. Symbols are the same as in Figures 22 and 23. (A color version of this figure is available in the online journal.)

7774 , and $7775 \AA$ A. At these wavelengths, line contamination is unlikely. We have corrected our O I triplet abundances for departures from LTE according to the prescriptions of Fabbian et al. (2009). These corrections, while certainly better than a pure LTE analysis, may still be imperfect, and in a few cases our stars span a wider parameter range than their grid $(4500 \mathrm{~K} \leqslant$ $T_{\text {eff }} \leqslant 6500 \mathrm{~K}, 2.0 \leqslant \log g \leqslant 5.0,-3.0 \leqslant[\mathrm{Fe} / \mathrm{H}] \leqslant 0$ ). Given that the trend is mostly seen for warm subgiants that do fall within the grid, this explanation alone hardly seems adequate. 

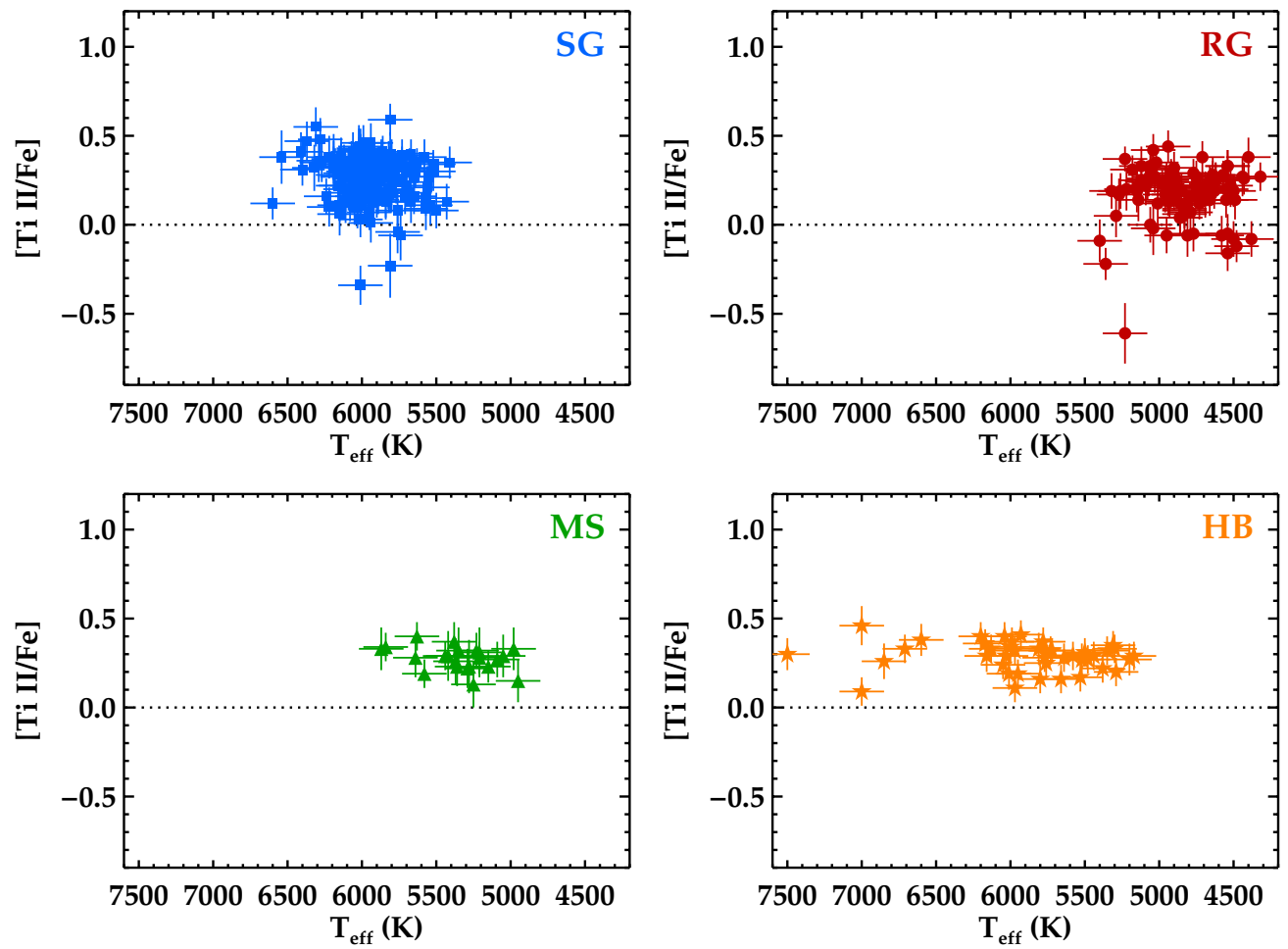

Figure 34. Derived $[\mathrm{Ti} / \mathrm{Fe}]$ ratios for ionized lines as a function of $T_{\text {eff. }}$. Symbols are the same as in Figures 22 and 23. (A color version of this figure is available in the online journal.)
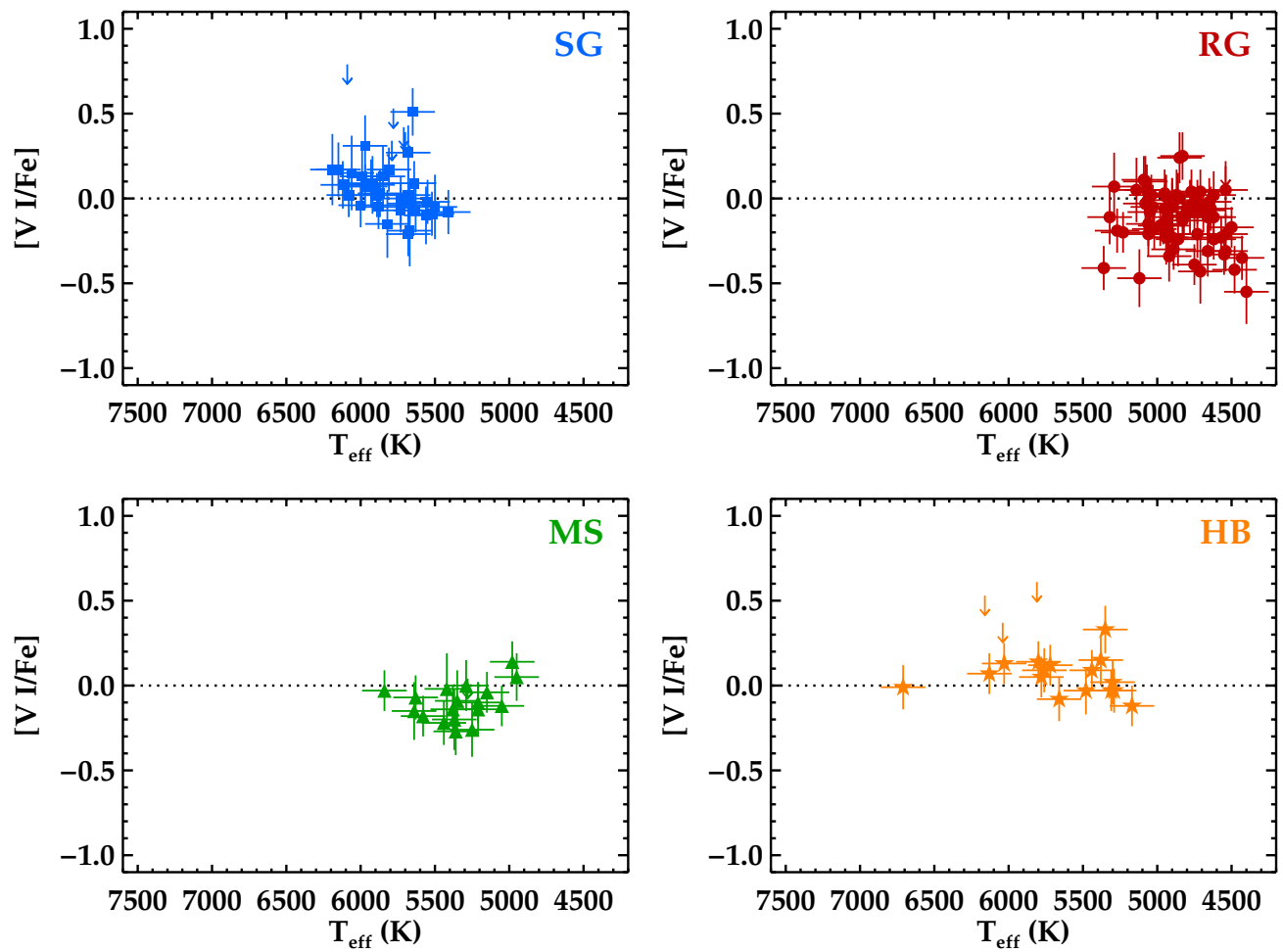

Figure 35. Derived $[\mathrm{V} / \mathrm{Fe}]$ ratios for neutral lines as a function of $T_{\text {eff. }}$. Symbols are the same as in Figures 22 and 23.

(A color version of this figure is available in the online journal.)

The Sc II, Ti I, V I, V II, Cr I, Mn I, Mn II, and Co I trends all run in the opposite sense from the Si I and O I trends. Most of these species are derived from many lines: the median number of lines used in the analysis for Ti I, Cr I, Mn I, Mn II, and Co I is eight, five, three, three, and three, respectively. VI and V II are each derived from one or two lines. For a trend of decreasing average abundance with decreasing $T_{\text {eff }}$, however, contamination by a molecular feature can be excluded. Thus it seems that the cause of the correlation in these cases is not unidentified blends. 

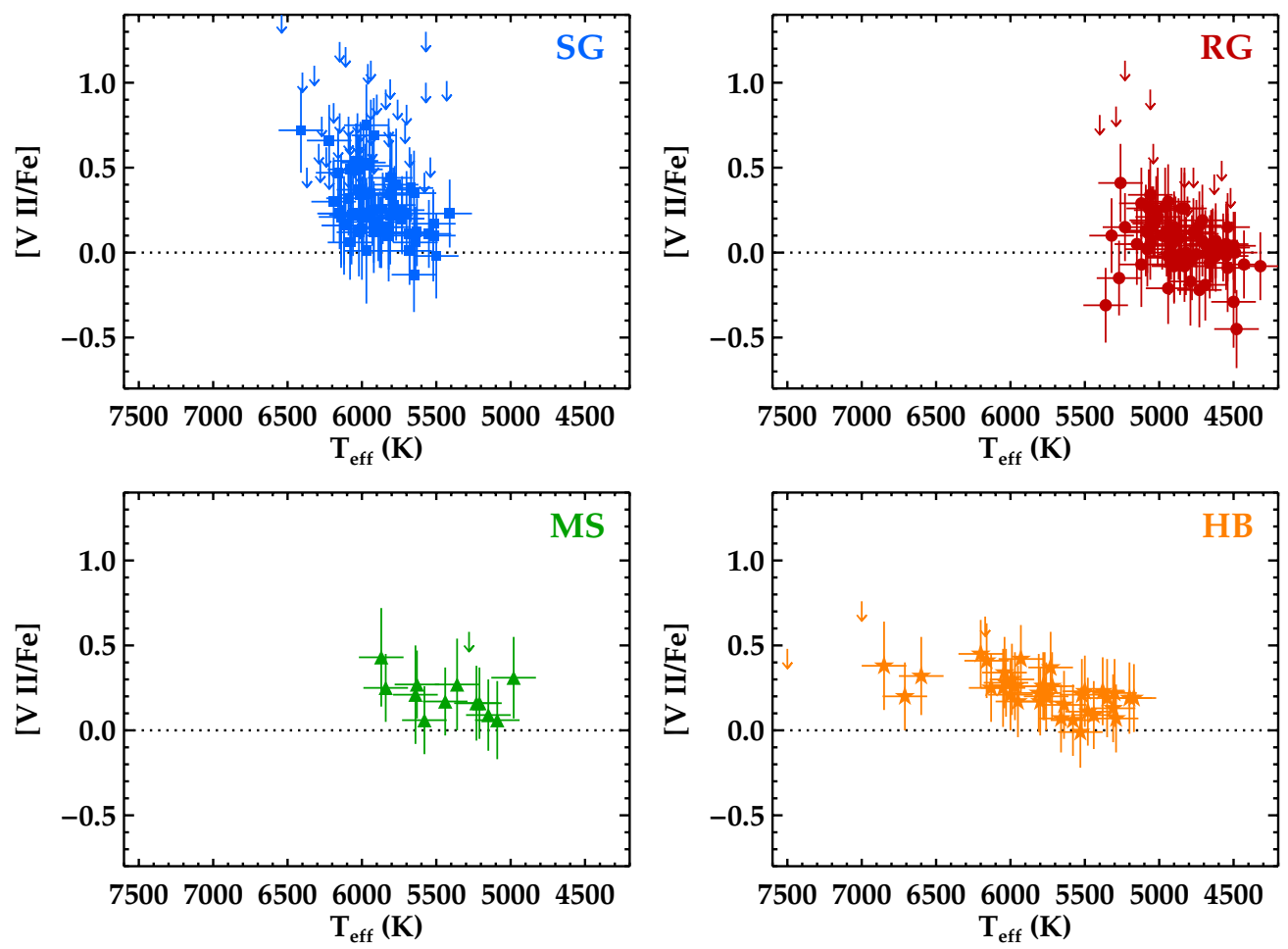

Figure 36. Derived $[\mathrm{V} / \mathrm{Fe}]$ ratios for ionized lines as a function of $T_{\text {eff }}$. Symbols are the same as in Figures 22 and 23. (A color version of this figure is available in the online journal.)
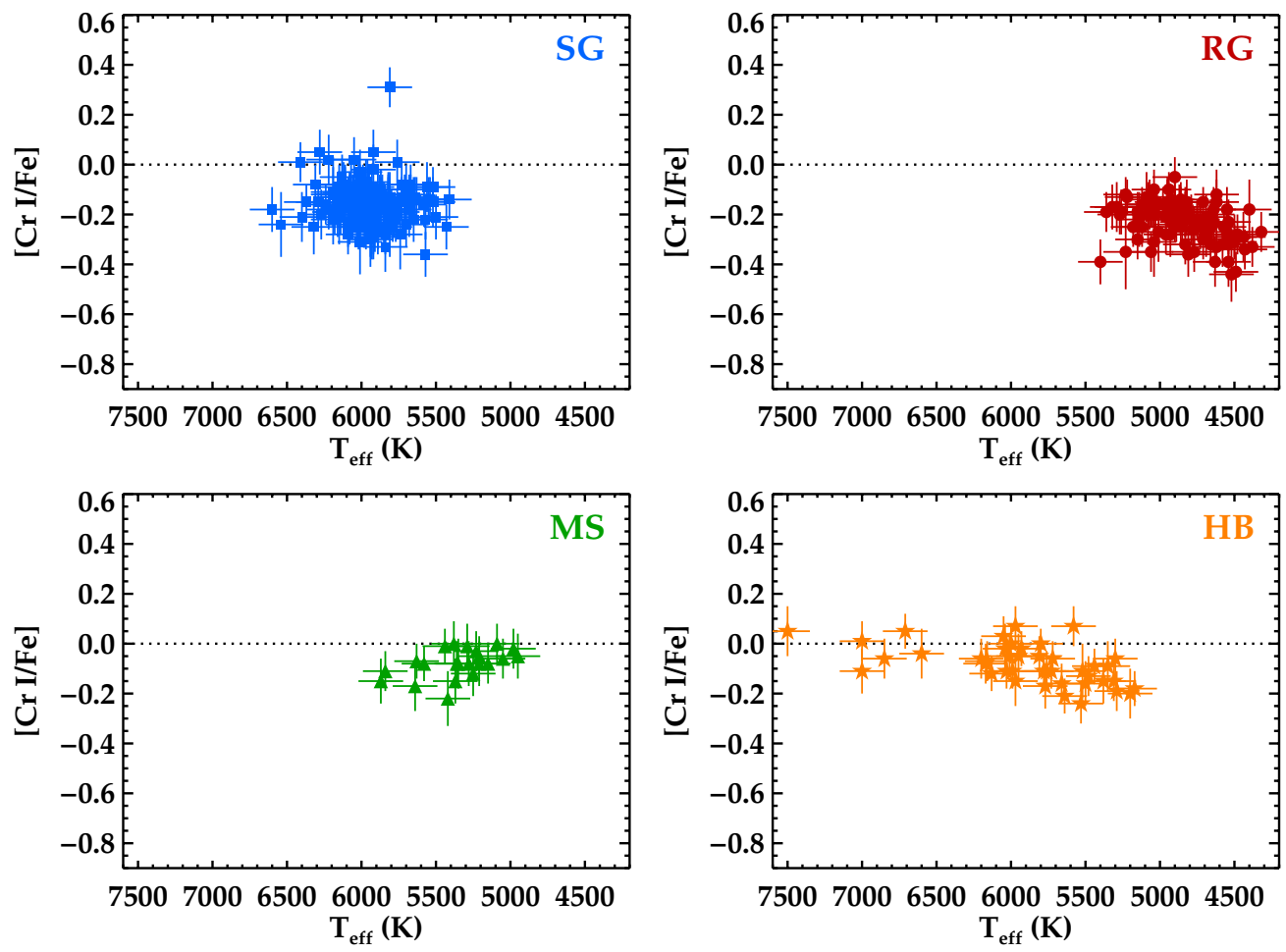

Figure 37. Derived $[\mathrm{Cr} / \mathrm{Fe}]$ ratios for neutral lines as a function of $T_{\text {eff. }}$. Symbols are the same as in Figures 22 and 23. (A color version of this figure is available in the online journal.)

We have included hfs components in our syntheses for all odd- $Z$ iron group species except $\mathrm{V}$ II, for which we are unable to locate published values for the hyperfine $\mathrm{A}$ and $\mathrm{B}$ constants for the levels of interest. In principle, this could lead to an overestimate of the VII abundance, especially for stars with stronger absorption lines. We have simulated possible ranges of broadening for the $\mathrm{V}_{\text {II lines up to }} 0.03 \AA$, which is larger than the broadening found for Sc II or Mn II. In a cool red giant star with EWs in the 80th percentile of strongest $V$ II lines for our sample, where neglecting the hfs might affect the abundance most 

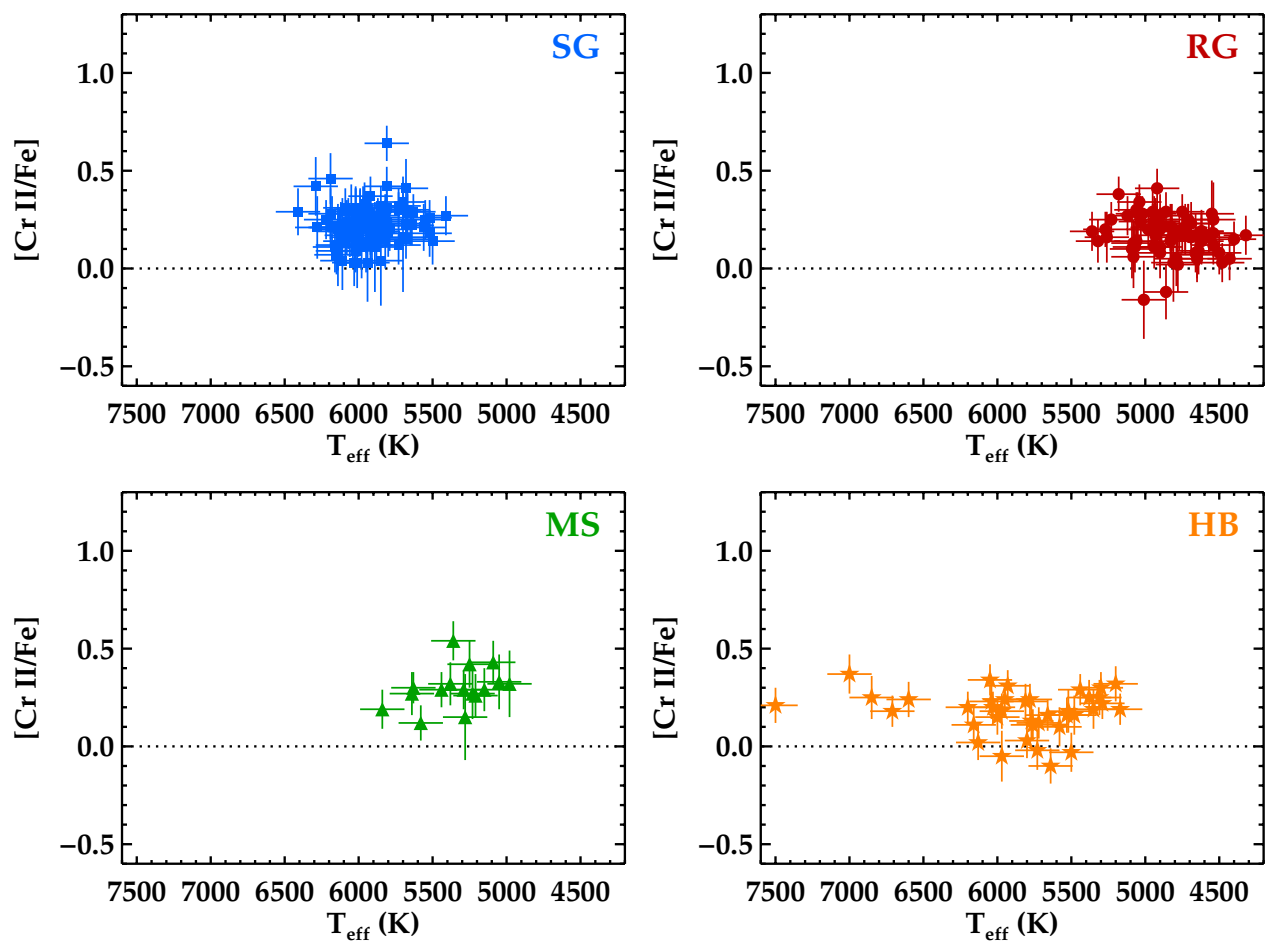

Figure 38. Derived $[\mathrm{Cr} / \mathrm{Fe}]$ ratios for ionized lines as a function of $T_{\text {eff. }}$. Symbols are the same as in Figures 22 and 23.

(A color version of this figure is available in the online journal.)
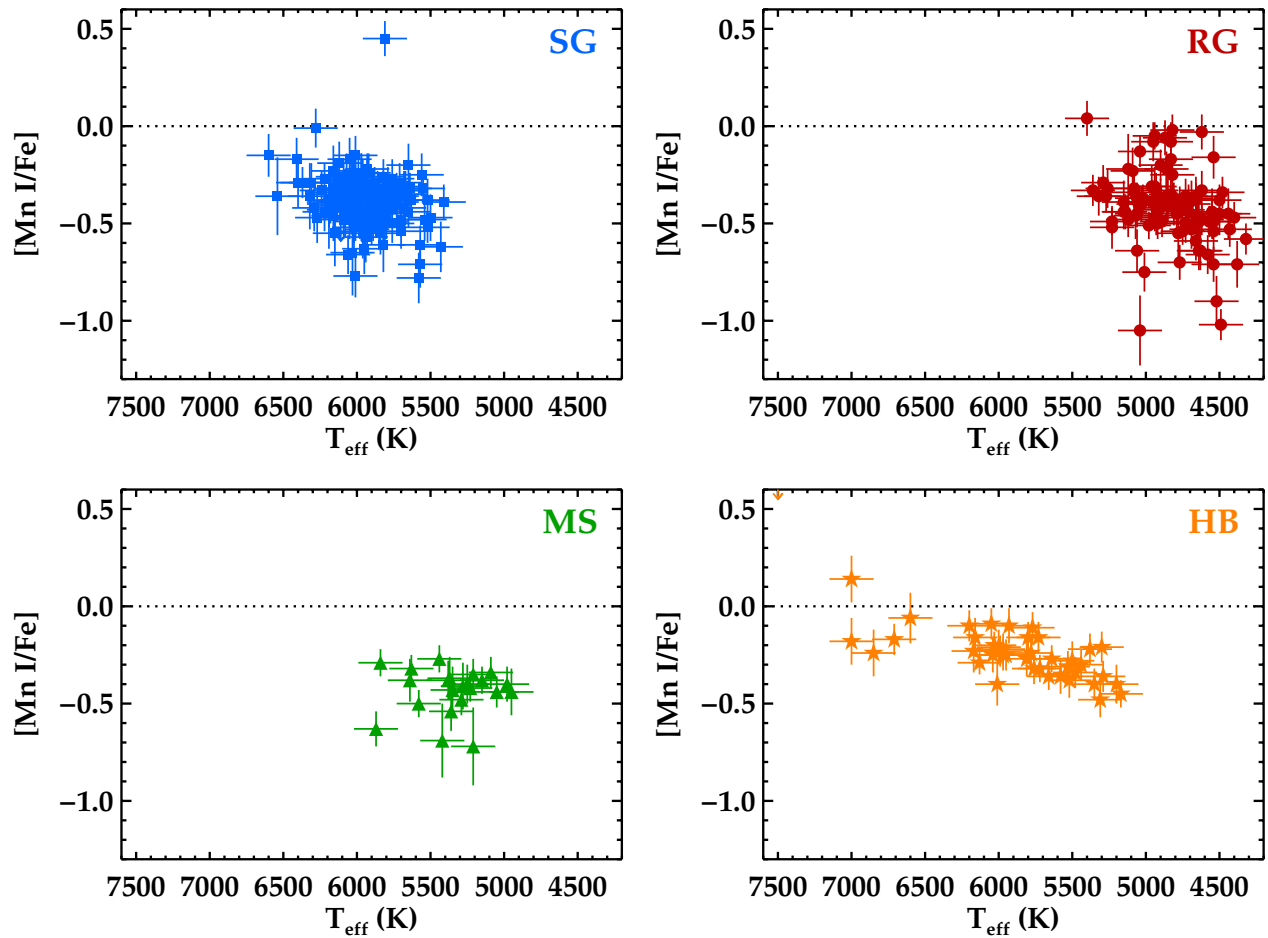

Figure 39. Derived $[\mathrm{Mn} / \mathrm{Fe}]$ ratios for neutral lines as a function of $T_{\text {eff. }}$. Symbols are the same as in Figures 22 and 23. (A color version of this figure is available in the online journal.)

significantly, we could potentially underestimate the vanadium abundance by $<0.1$ dex. Regardless, this correction goes in the wrong direction. Cooler stars with stronger absorption lines should yield higher abundances when neglecting hfs. Therefore neglecting the $\mathrm{hfs}$ for ${ }^{51} \mathrm{~V}$ is not the source of the $\mathrm{V}_{\text {II }}$ trend with $T_{\text {eff }}$.
In Figures 52 through 55 we show the [Ti II/Ti I], [V II/V I], [Cr II/Cr I] and [Mn II/Mn I] ratios plotted as functions of $T_{\text {eff }}$. We remind readers that these ratios denote the average total abundance of each element as derived from the ionized or neutral species after ionization corrections assuming LTE have been applied. None shows any trend with $T_{\text {eff }}$. 

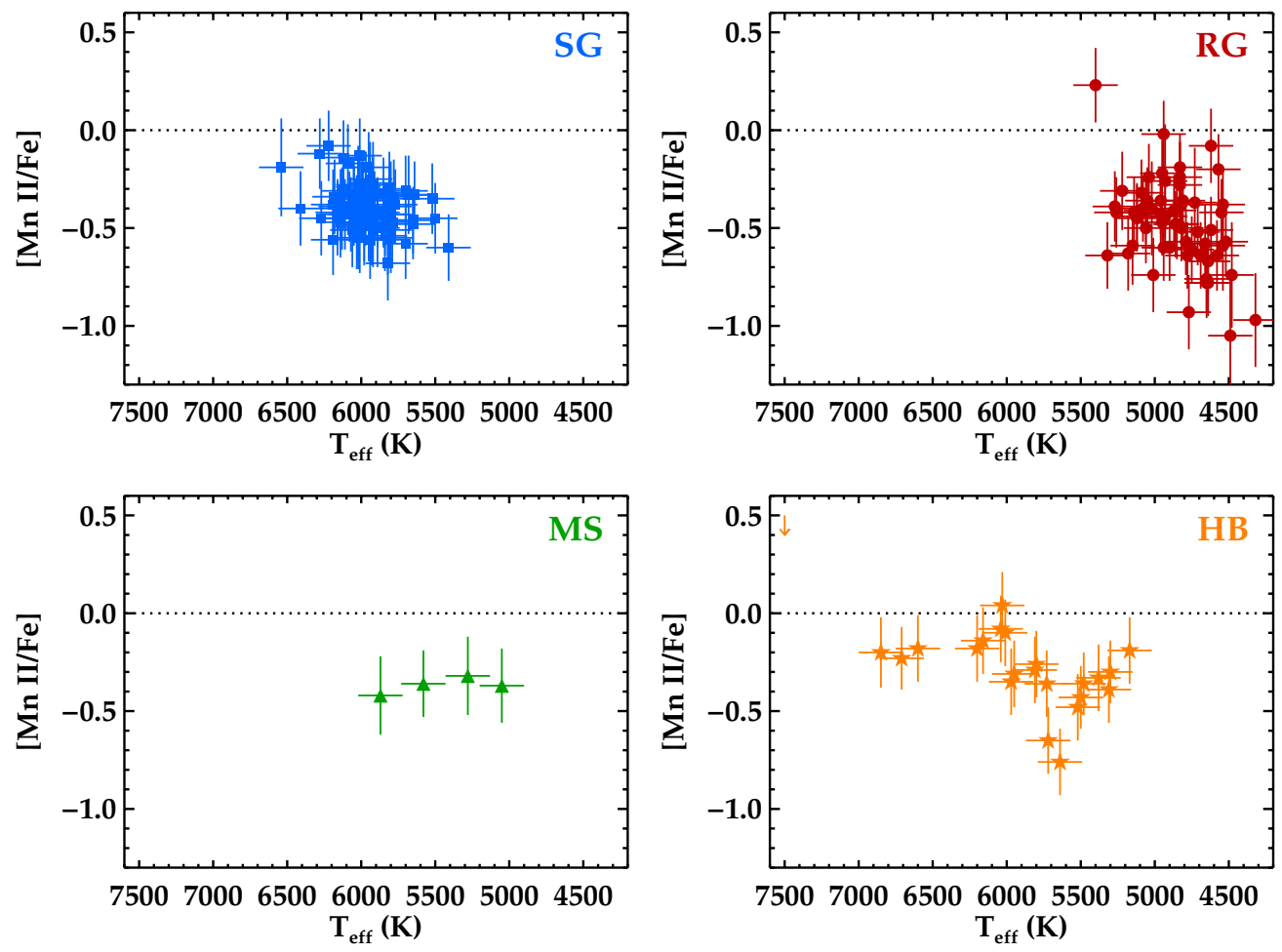

Figure 40. Derived $[\mathrm{Mn} / \mathrm{Fe}]$ ratios for ionized lines as a function of $T_{\mathrm{eff}}$. Symbols are the same as in Figures 22 and 23. (A color version of this figure is available in the online journal.)

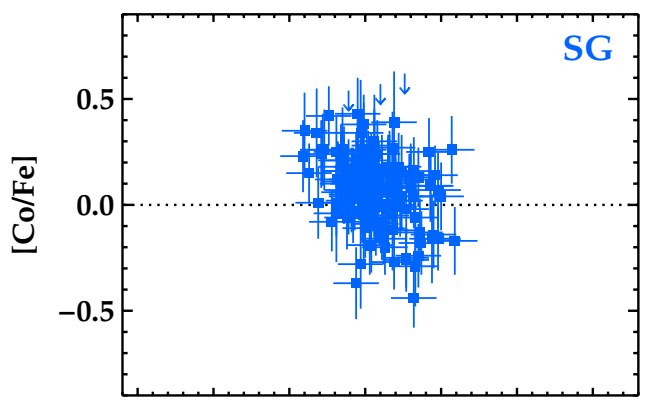

7500700065006000550050004500 $\mathrm{T}_{\text {eff }}(\mathrm{K})$

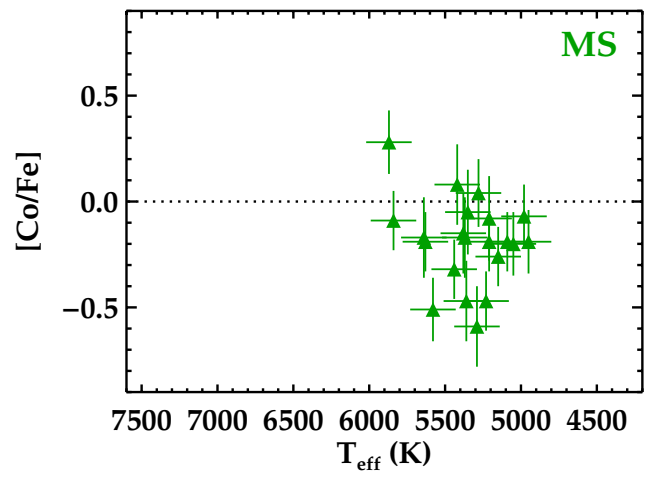

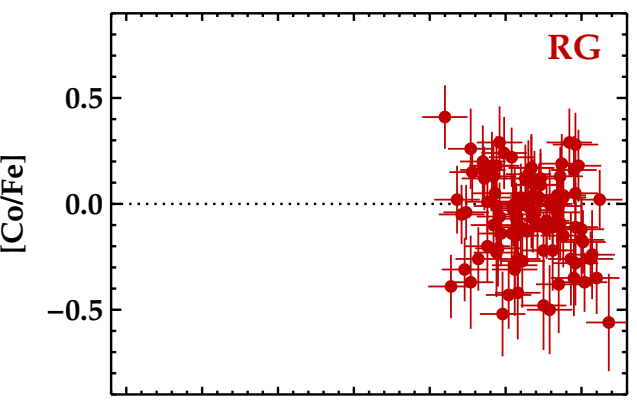

7500700065006000550050004500 $\mathbf{T}_{\text {eff }}(\mathbf{K})$

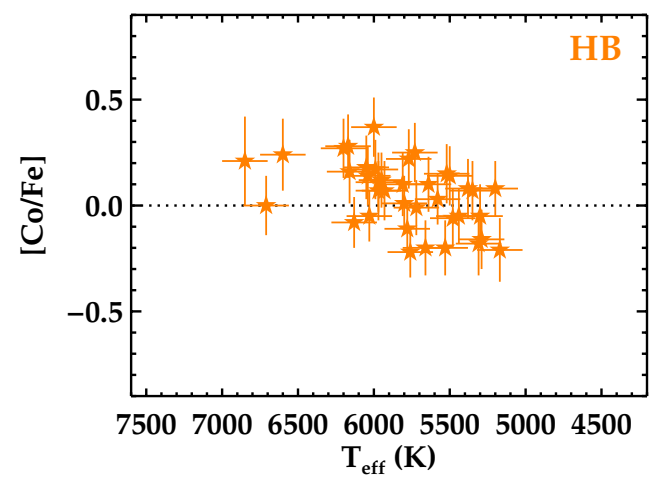

Figure 41. Derived $[\mathrm{Co} / \mathrm{Fe}]$ ratios as a function of $T_{\mathrm{eff}}$. Symbols are the same as in Figures 22 and 23.

(A color version of this figure is available in the online journal.)

[Ti II/Ti I] and [V II/V I] show a significant trend when plotted as a function of metallicity, as shown in Figures 56 and 57. These ratios increase with increasing metallicity. This trend is present in stars in each of the different evolutionary classes, though the magnitude of the slope differs. One possible expla- nation is that Saha equilibrium is an inadequate description of the ionization distribution for stars in our sample. [Cr II/CrI] shows no correlation with metallicity (Figure 58), and [Mn II/Mn I] shows, at most, a weak trend among the SG class only (Figure 59). 

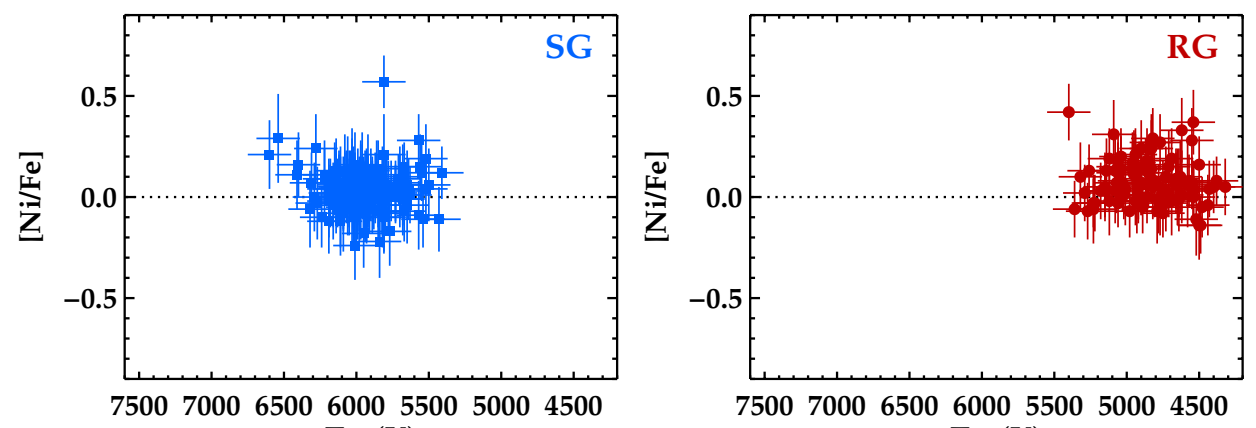

$\mathrm{T}_{\text {eff }}(\mathrm{K})$

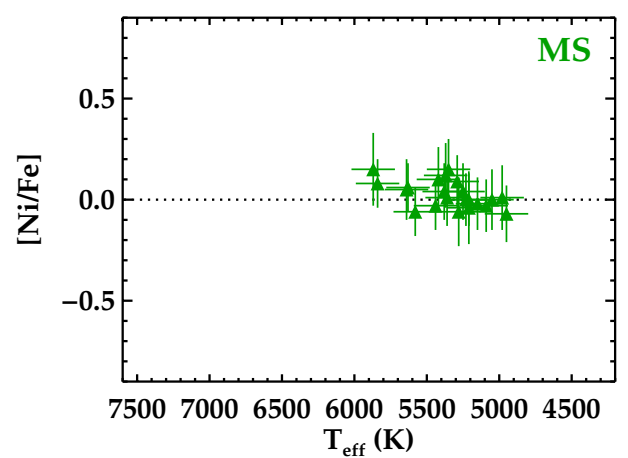

7500700065006000550050004500

$\mathrm{T}_{\text {eff }}(\mathrm{K})$

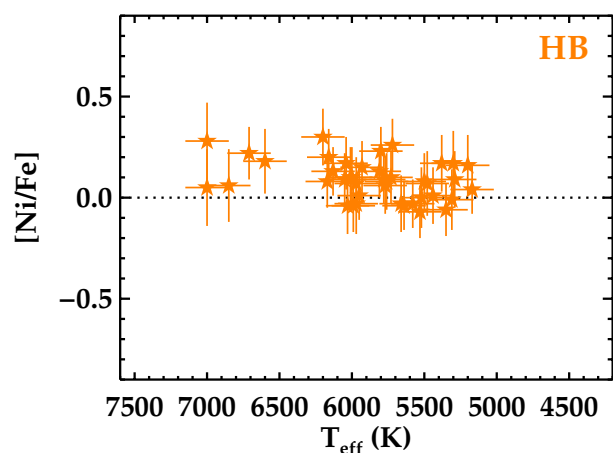

Figure 42. Derived $[\mathrm{Ni} / \mathrm{Fe}]$ ratios as a function of $T_{\text {eff }}$. Symbols are the same as in Figures 22 and 23.

(A color version of this figure is available in the online journal.)
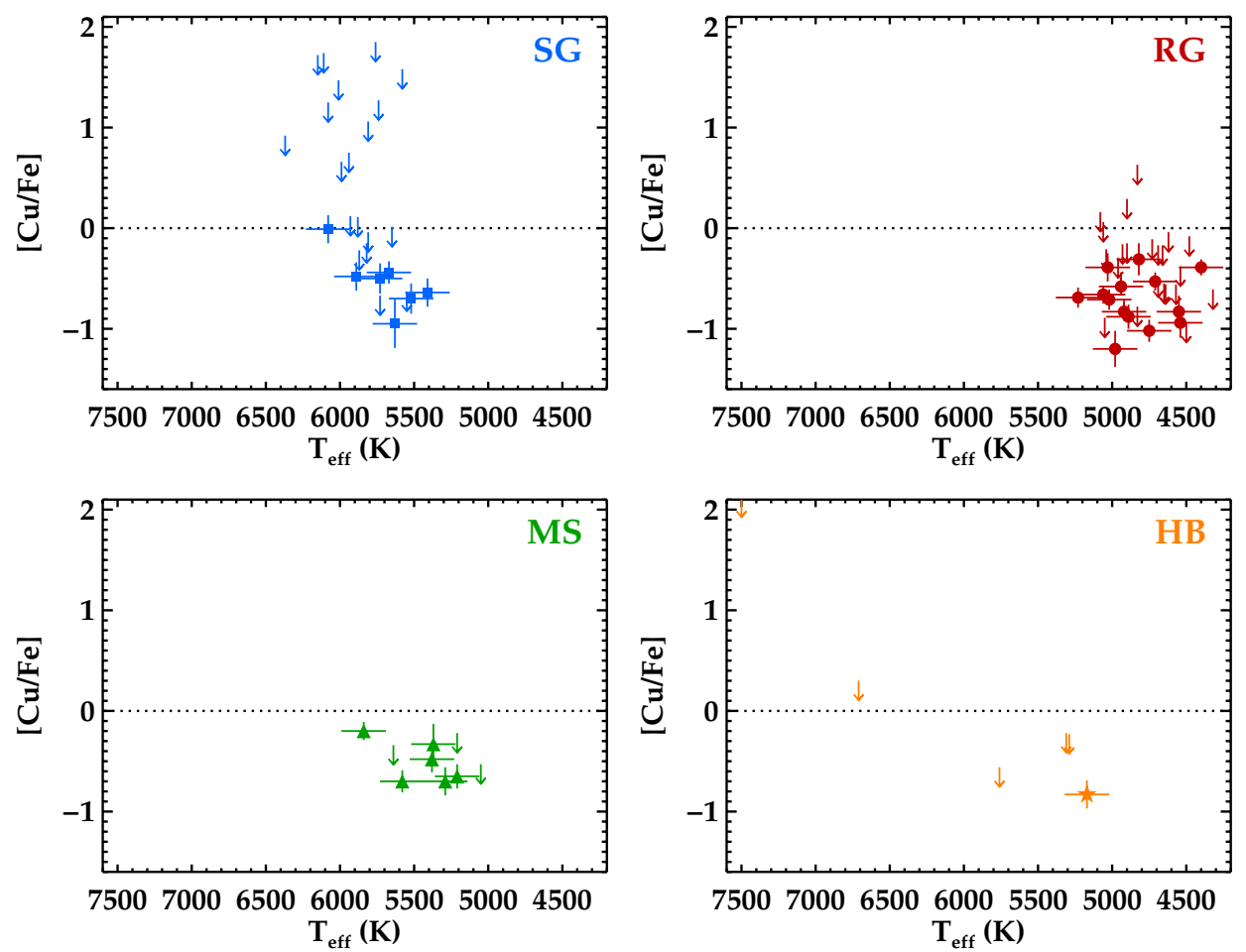

Figure 43. Derived $[\mathrm{Cu} / \mathrm{Fe}]$ ratios as a function of $T_{\text {eff }}$. Symbols are the same as in Figures 22 and 23.

(A color version of this figure is available in the online journal.)

In conclusion, we cannot offer explanations for all of the non-zero abundance trends with $T_{\text {eff }}$. We urge those who wish to make use of our abundances to be careful with the species discussed in this section. One approach to mitigate the influence of these effects is to consider abundances of stars in only a limited range of $T_{\text {eff }},[\mathrm{Fe} / \mathrm{H}]$, and evolutionary state. Our sample of 313 stars is large enough that sampling narrow ranges of parameter space still provides satisfactory numbers for statistical comparison in most cases.

\section{SUMMARY}

This paper presents the technical details of our analysis to measure EWs, radial velocities, derive model atmosphere 

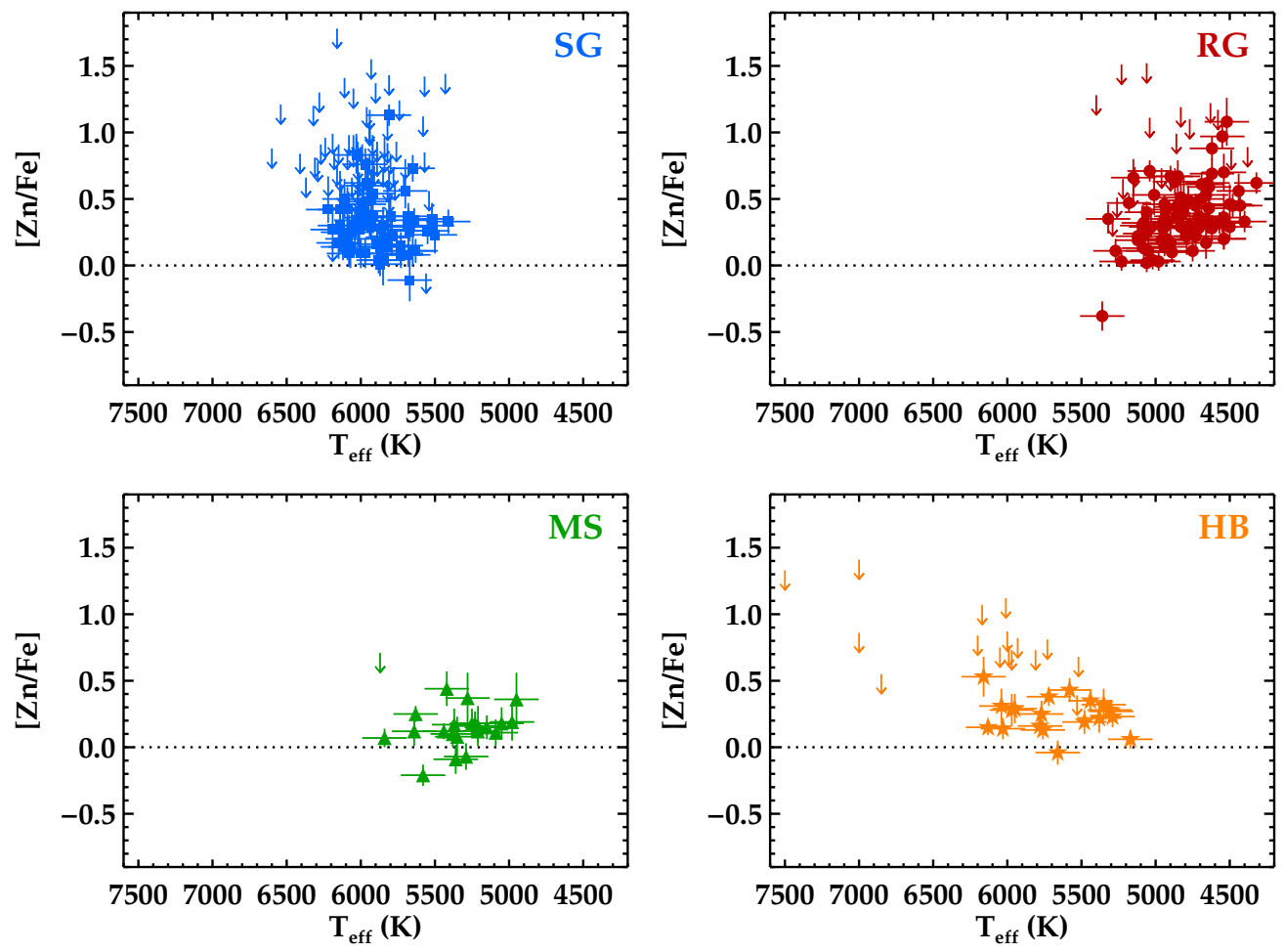

Figure 44. Derived $[\mathrm{Zn} / \mathrm{Fe}]$ ratios as a function of $T_{\text {eff }}$. Symbols are the same as in Figures 22 and 23.

(A color version of this figure is available in the online journal.)
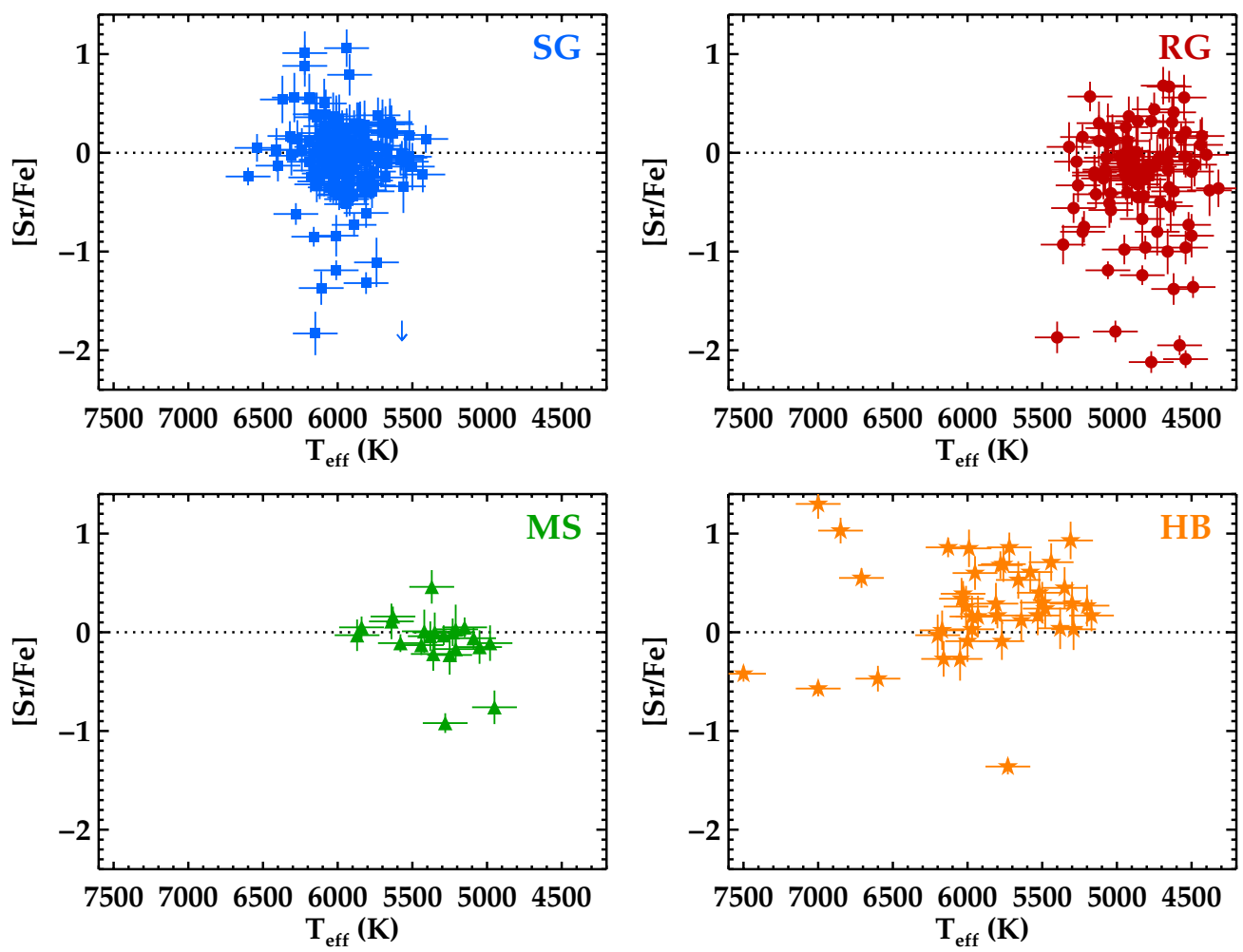

Figure 45. Derived [ $\mathrm{Sr} / \mathrm{Fe}]$ ratios as a function of $T_{\text {eff }}$. Symbols are the same as in Figures 22 and 23.

(A color version of this figure is available in the online journal.)

parameters, and derive chemical abundances from hundreds of individual high resolution spectroscopic observations of metalpoor halo stars. Abundances or upper limits are reported for 53 species of 48 elements in 313 metal-poor stars. Our analysis finds 19 stars with metallicities $[\mathrm{Fe} / \mathrm{H}] \leqslant-3.5,84$ stars with $[\mathrm{Fe} / \mathrm{H}] \leqslant-3.0$, and 210 stars with $[\mathrm{Fe} / \mathrm{H}] \leqslant-2.5$. For the stars selected from the HK Survey, the numbers of stars below these three metallicity thresholds are 15, 67, and 173, respectively. 

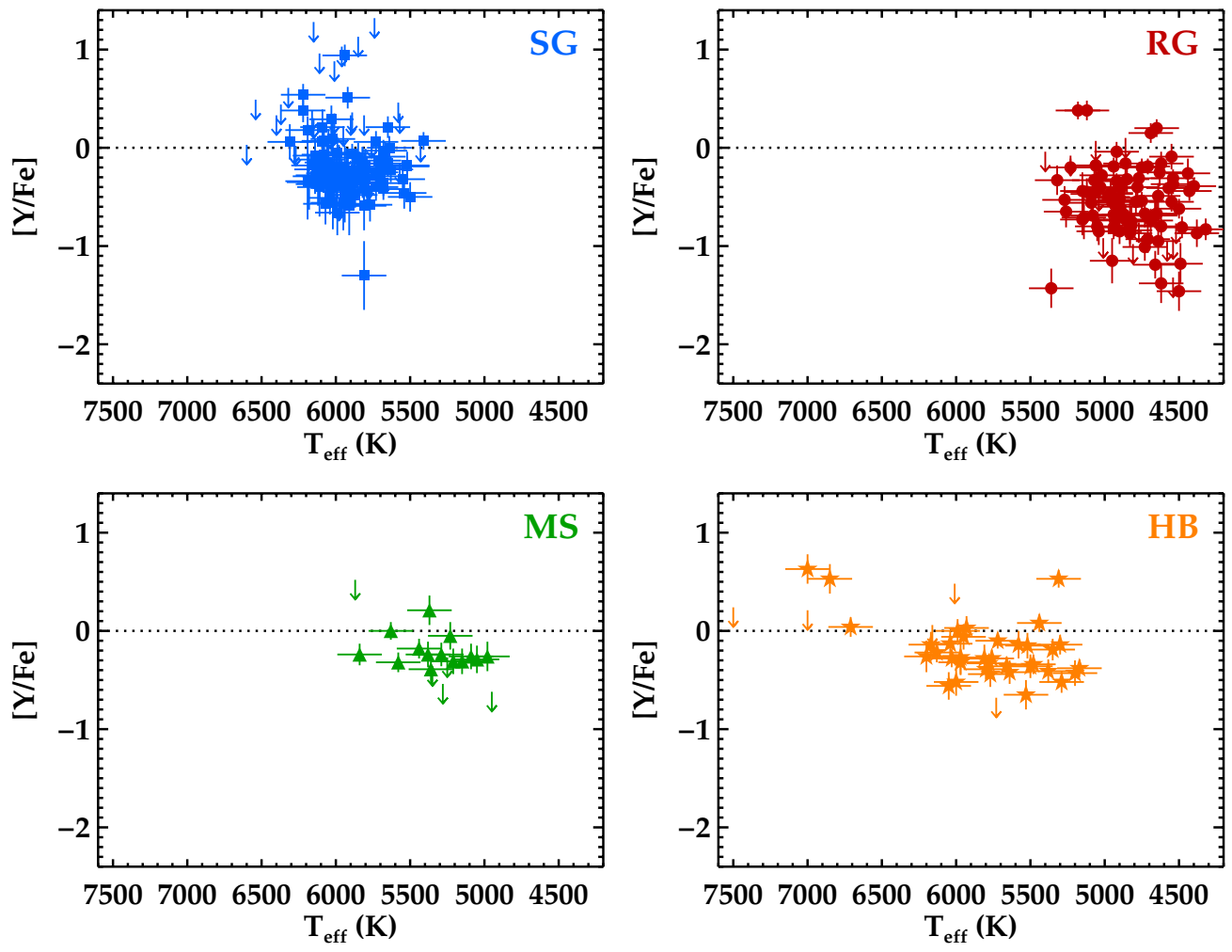

Figure 46. Derived [Y/Fe] ratios as a function of $T_{\text {eff }}$. Symbols are the same as in Figures 22 and 23.

(A color version of this figure is available in the online journal.)
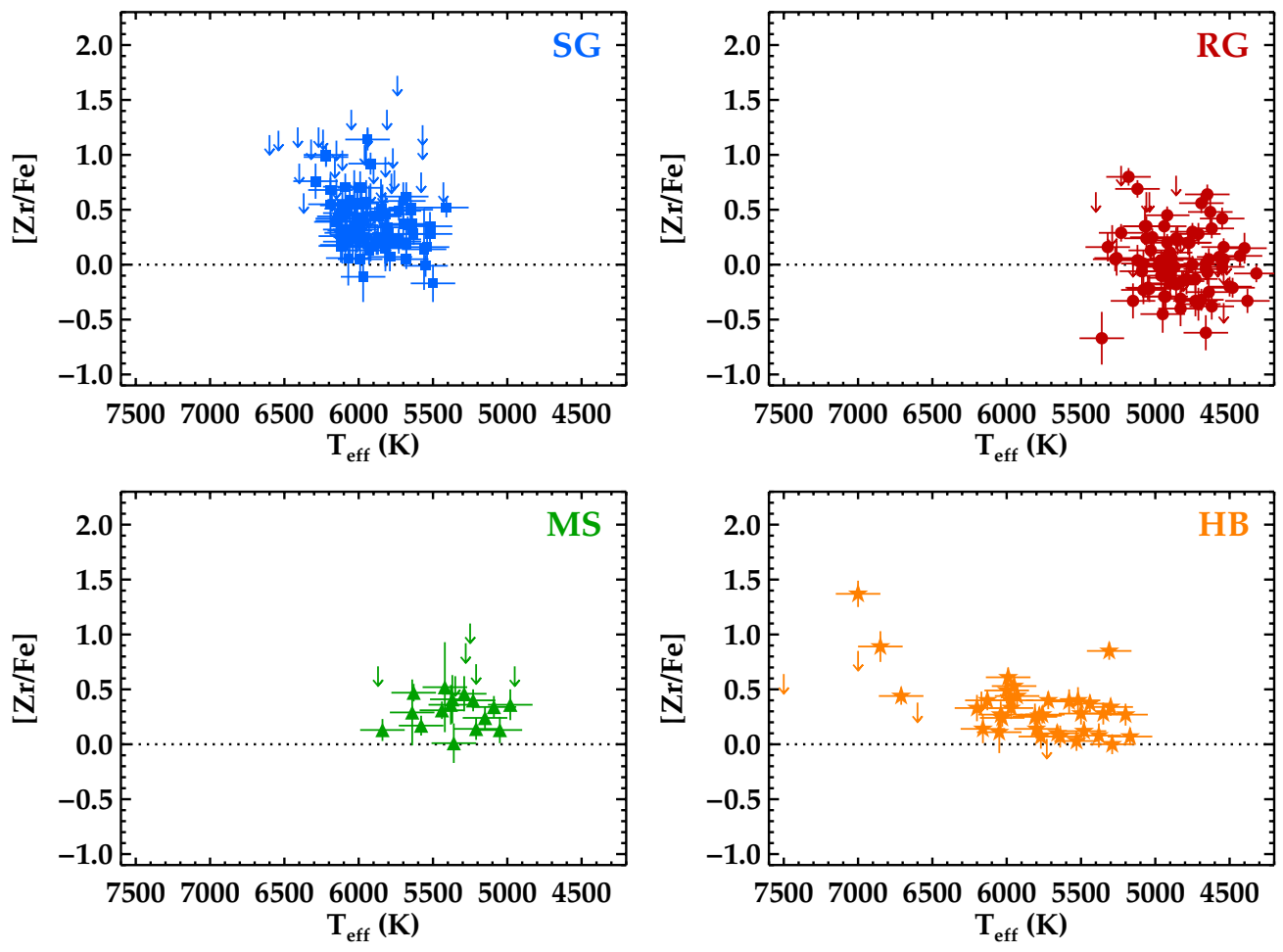

Figure 47. Derived $[\mathrm{Zr} / \mathrm{Fe}]$ ratios as a function of $T_{\text {eff }}$. Symbols are the same as in Figures 22 and 23.

(A color version of this figure is available in the online journal.)

In subsequent papers, we will discuss the interpretation of these abundances regarding the chemical evolution of the Galactic halo and stellar nucleosynthesis in the early universe. We welcome other investigators to make use of these results in their own work; a few words of caution, however, are appropriate.

First, our analysis is performed assuming that LTE holds in the line-forming layers of the photosphere. We employ static, 1D, 

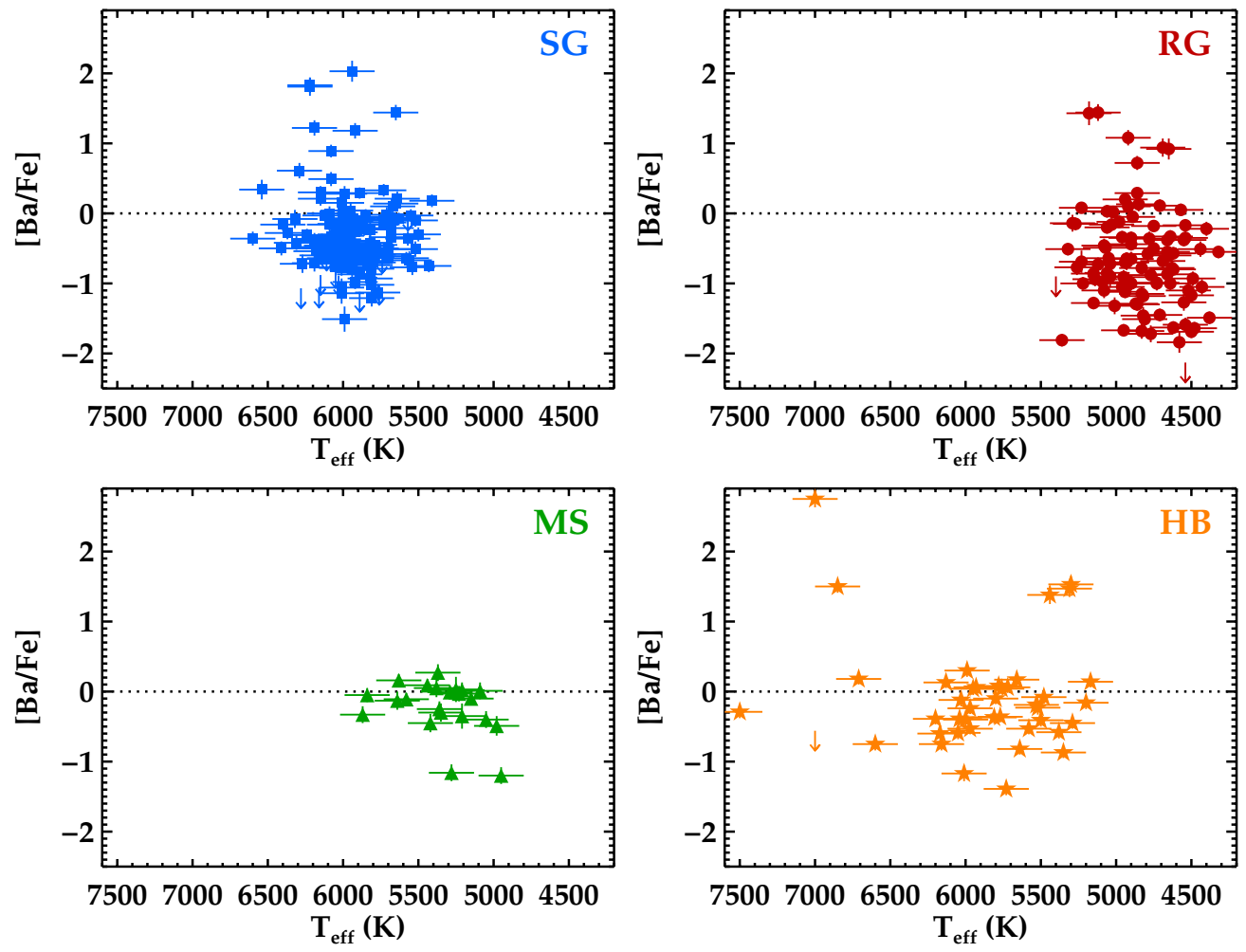

Figure 48. Derived $[\mathrm{Ba} / \mathrm{Fe}]$ ratios as a function of $T_{\text {eff }}$. Symbols are the same as in Figures 22 and 23.

(A color version of this figure is available in the online journal.)

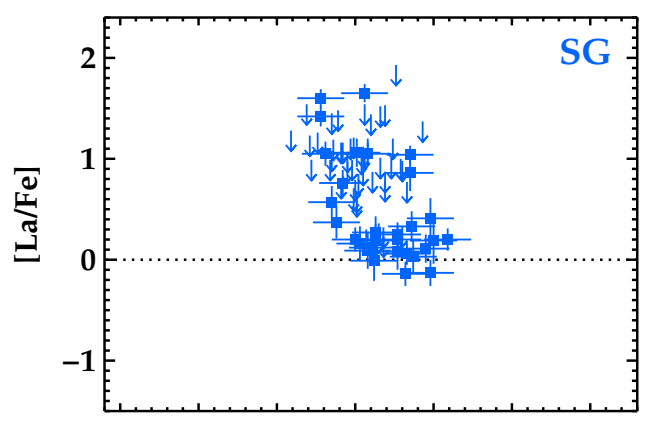

7500700065006000550050004500 $\mathrm{T}_{\text {eff }}(\mathrm{K})$

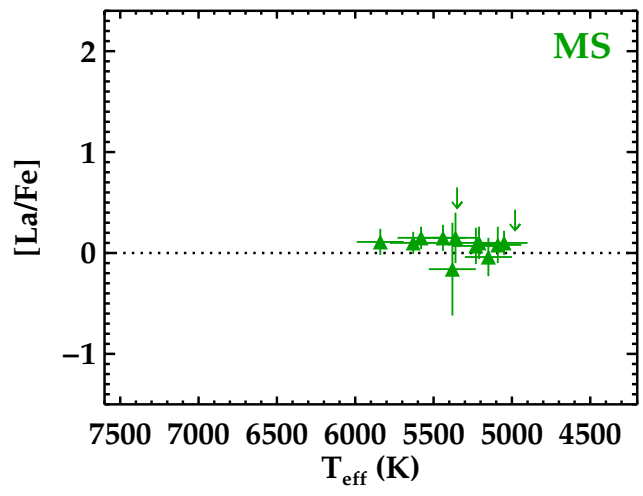

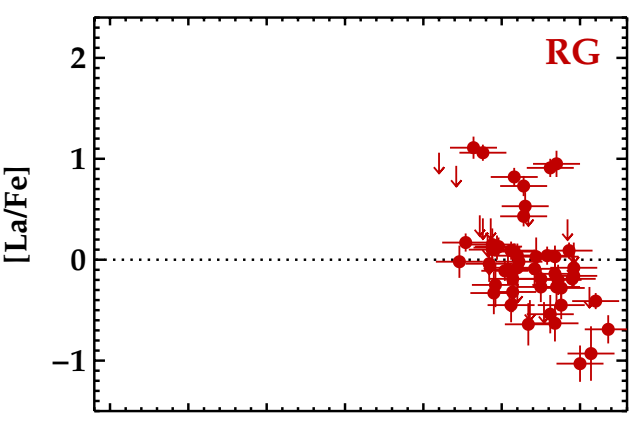

7500700065006000550050004500 $\mathrm{T}_{\text {eff }}(\mathrm{K})$

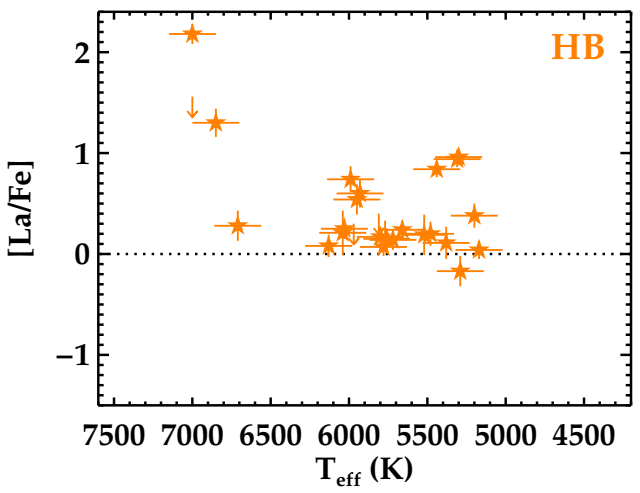

Figure 49. Derived $[\mathrm{La} / \mathrm{Fe}]$ ratios as a function of $T_{\text {eff }}$. Symbols are the same as in Figures 22 and 23.

(A color version of this figure is available in the online journal.)

plane-parallel model atmospheres constructed assuming LTE for a fixed set of abundances. To relax these assumptions would require substantial increases in computing power and relevant atomic data, and it is currently not practical to do so for a survey of this scale. Our results will differ, of course, from those computed using such techniques for individual stars.

Second, as discussed in detail in Section 8.3, our metallicity scale is slightly lower than that found by previous investigations 

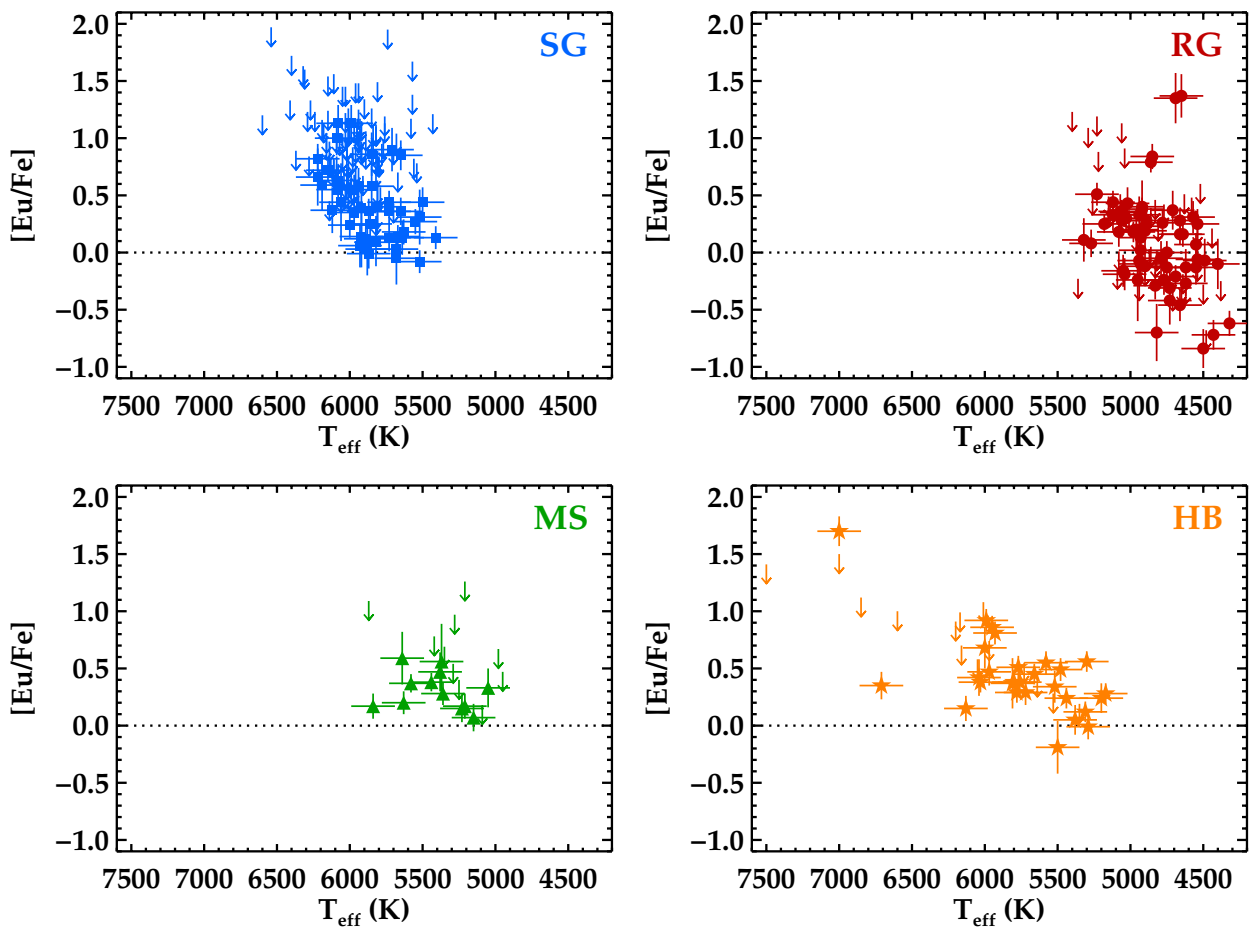

Figure 50. Derived $[\mathrm{Eu} / \mathrm{Fe}]$ ratios as a function of $T_{\mathrm{eff}}$. Symbols are the same as in Figures 22 and 23.

(A color version of this figure is available in the online journal.)
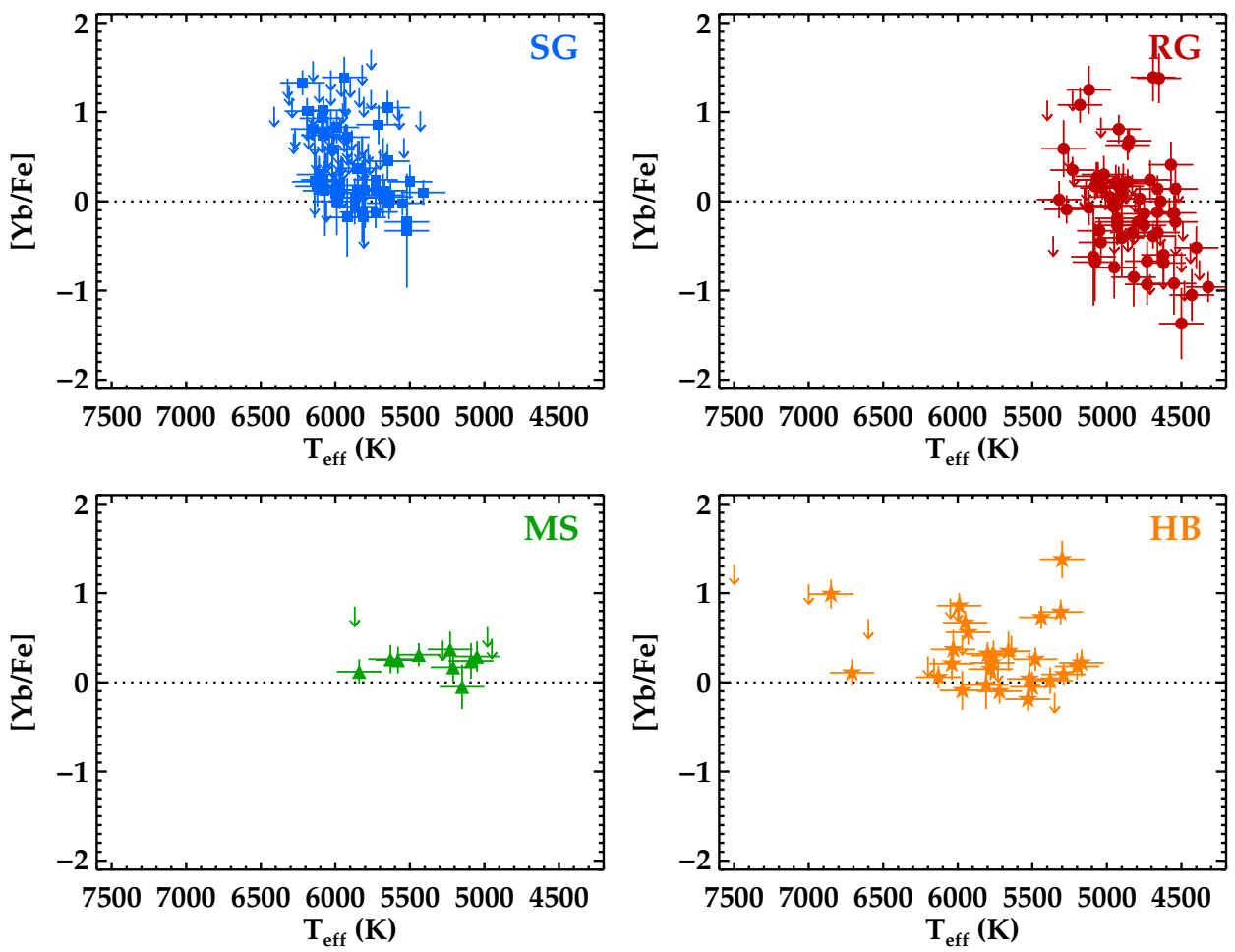

Figure 51. Derived [ $\mathrm{Yb} / \mathrm{Fe}]$ ratios as a function of $T_{\text {eff }}$. Symbols are the same as in Figures 22 and 23.

(A color version of this figure is available in the online journal.)

of stars in common. We have derived most model atmosphere parameters by spectroscopic methods, whereas recent abundance studies of extremely metal-poor stars use a combination of photometric and spectroscopic methods to derive these quantities. A natural consequence of this approach is that our derived metallicities are, on average, lower by $\approx 0.25$ dex for red giants and $\approx 0.04$ dex for subgiants in common with previous studies. The mean metallicity differences are a function of the evolutionary state, as reported in Table 9.

Third, this is a biased sample, and the biases are not easily quantified. We have drawn our targets from a variety of sources, and even those selected from the HK Survey are a heterogeneous 

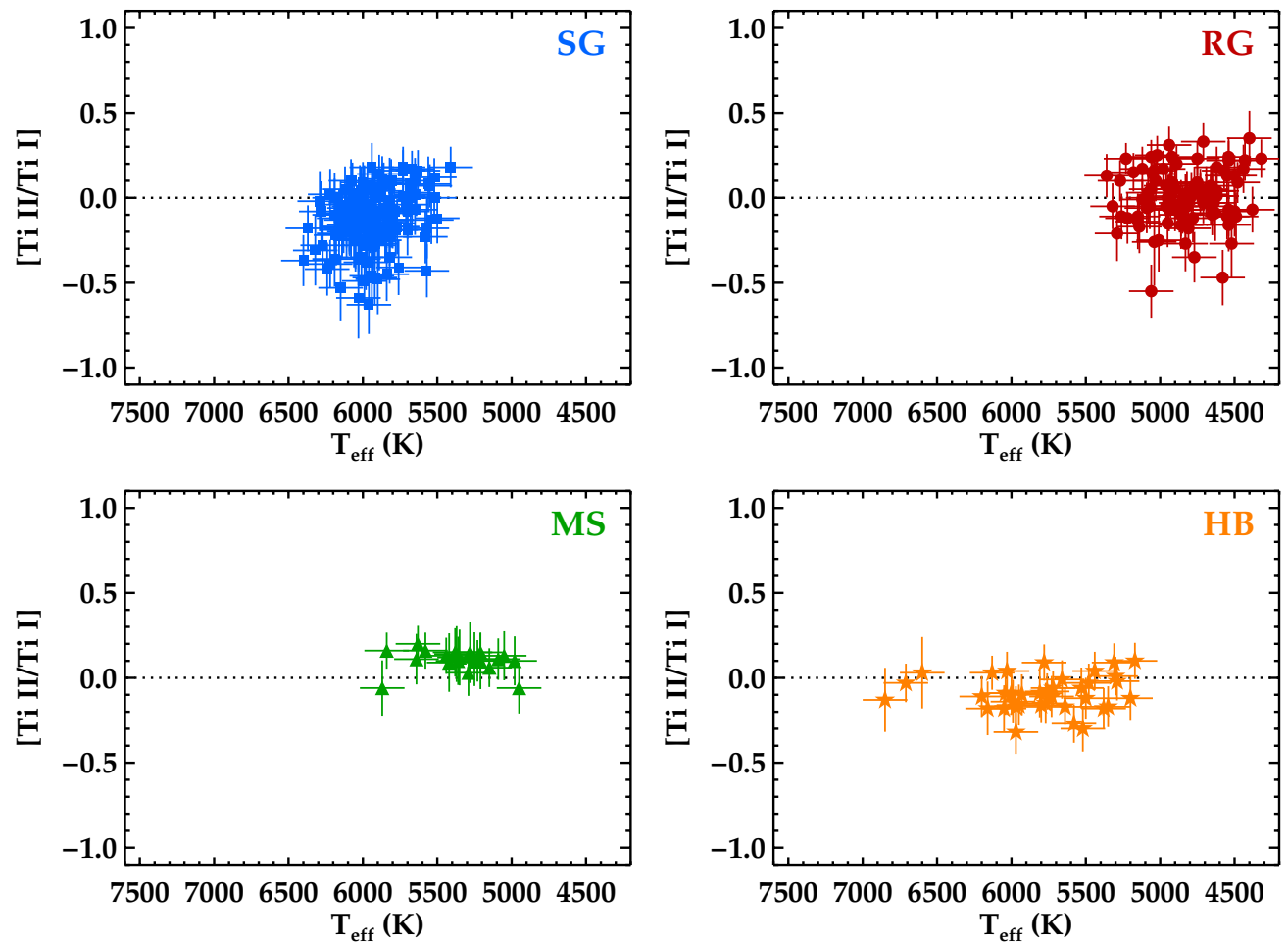

Figure 52. Ratios of the total titanium abundance derived from each of the ionized and neutral species as a function of $T_{\text {eff }}$. Each star is displayed only if both species have been detected. Symbols are the same as in Figures 22 and 23.

(A color version of this figure is available in the online journal.)
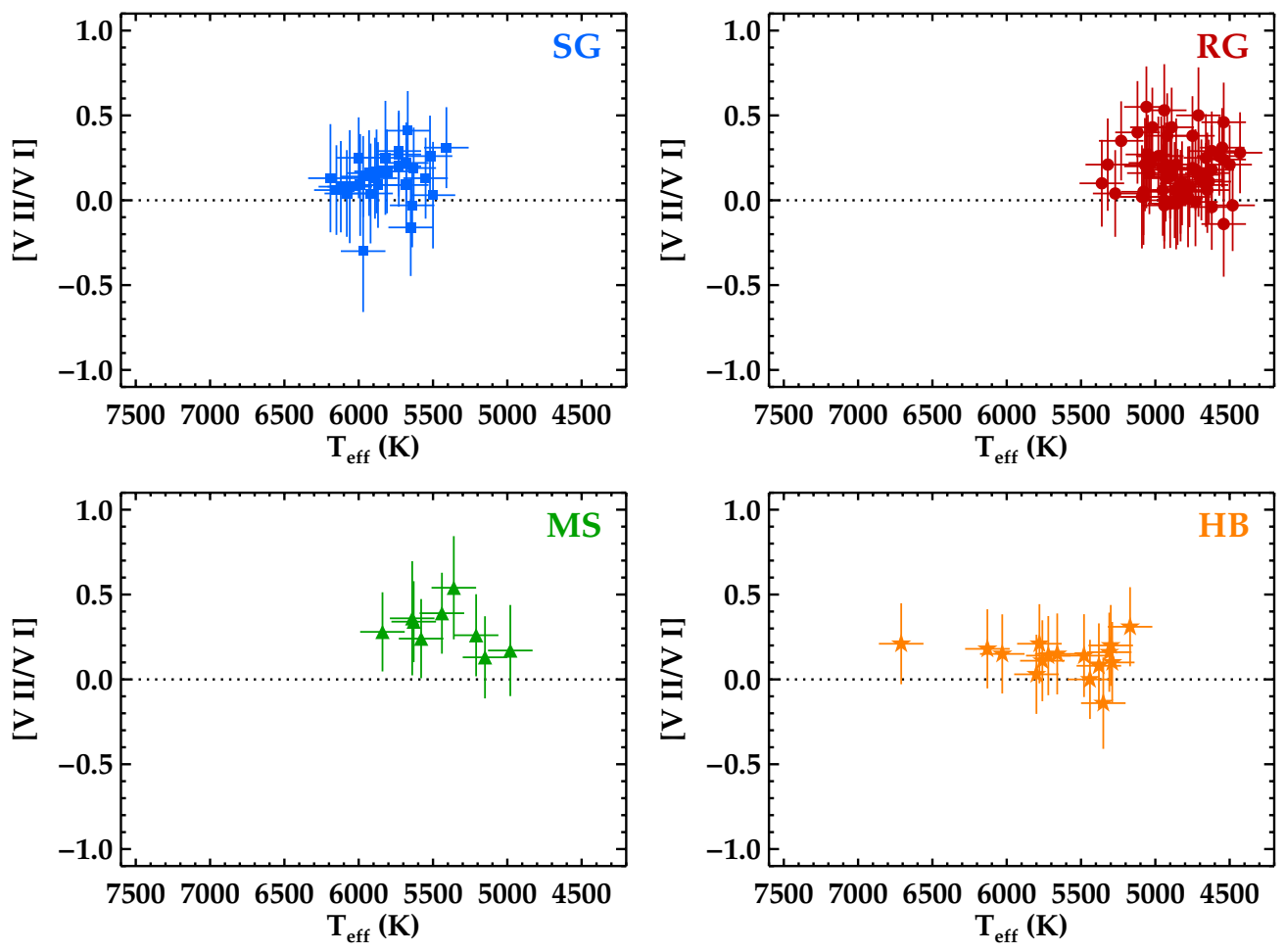

Figure 53. Ratios of the total vanadium abundance derived from each of the ionized and neutral species as a function of $T_{\text {eff. }}$ Each star is displayed only if both species have been detected. Symbols are the same as in Figures 22 and 23.

(A color version of this figure is available in the online journal.)

sample where stars we deem to be chemically interesting (based on previous studies) are overrepresented. Efforts to reconstruct the metallicity distribution function or estimate the frequency of carbon-enhanced stars, for example, using these data alone are not advised. Our data can be used, however, to calibrate other samples whose biases are well quantified.

Finally, some elemental ratios show a dependence on the stellar evolutionary state. For this reason, we strongly urge users to 

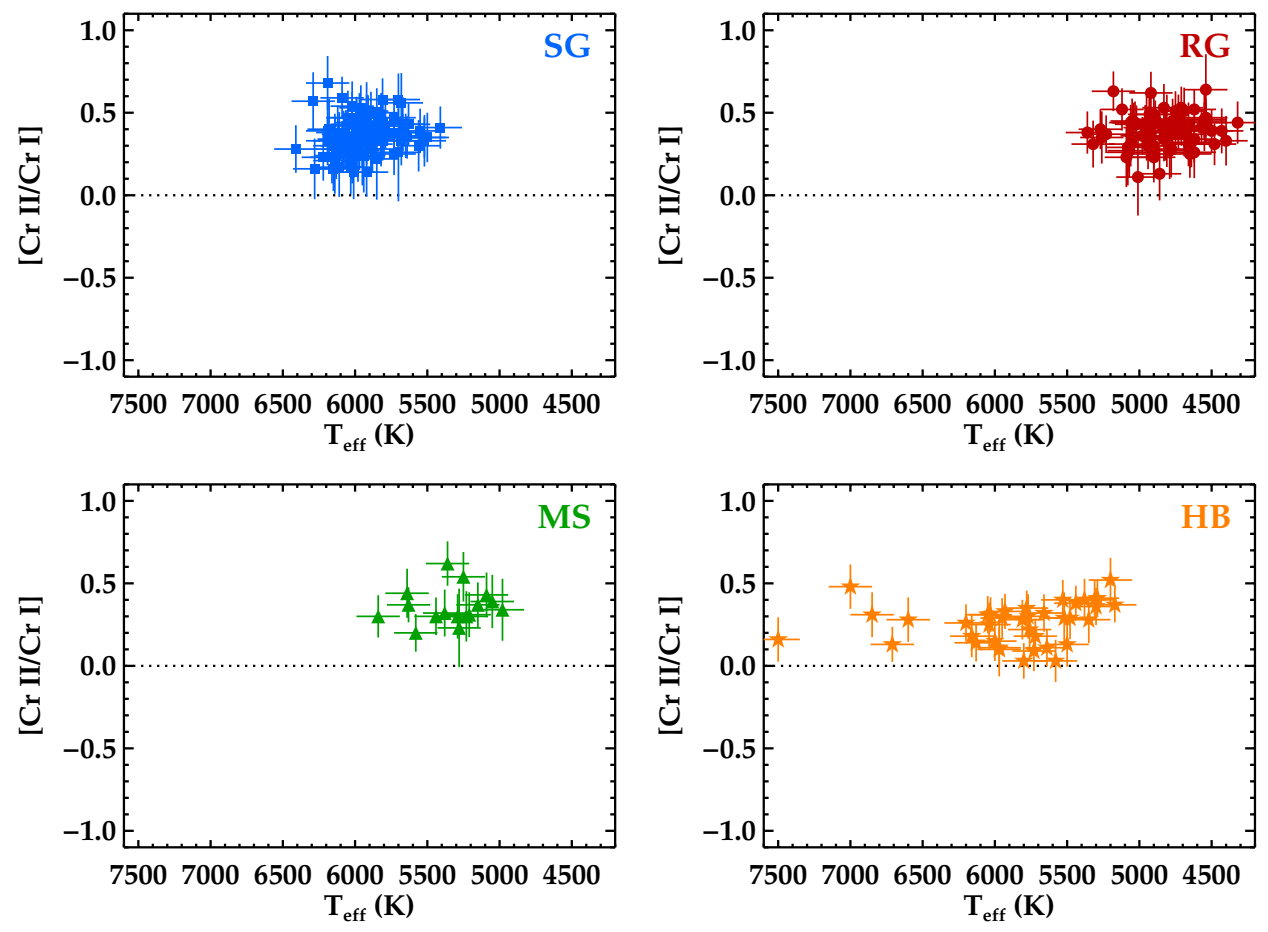

Figure 54. Ratios of the total chromium abundance derived from each of the ionized and neutral species as a function of $T_{\text {eff }}$. Each star is displayed only if both species have been detected. Symbols are the same as in Figures 22 and 23.

(A color version of this figure is available in the online journal.)
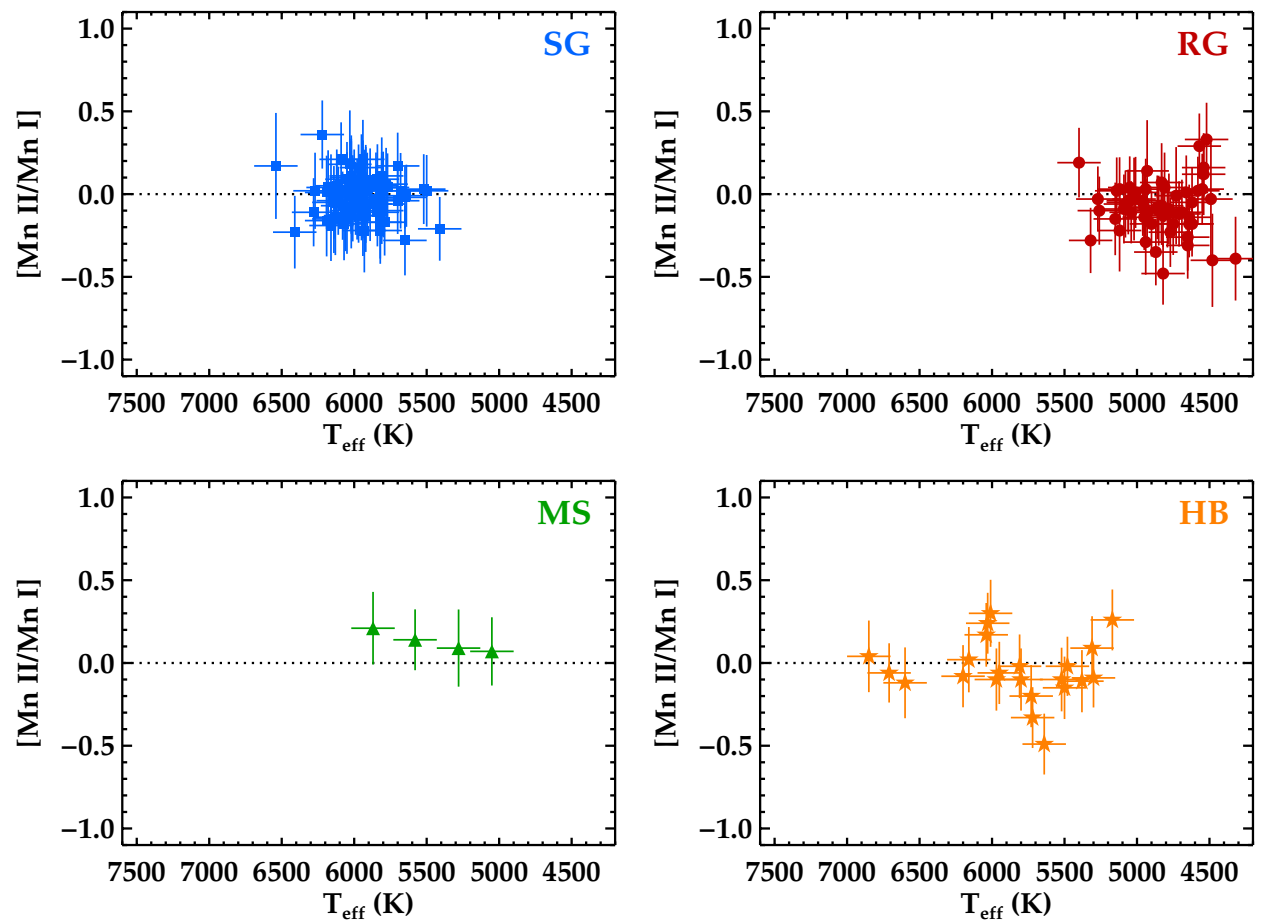

Figure 55. Ratios of the total manganese abundance derived from each of the ionized and neutral species as a function of $T_{\text {eff }}$. Each star is displayed only if both species have been detected. Symbols are the same as in Figures 22 and 23.

(A color version of this figure is available in the online journal.)

avoid plotting abundances of all 313 stars on the same diagram when detailed comparisons are intended. Instead, the size of this sample may be exploited to minimize systematic errors arising from the analysis techniques. For example, it is possible to select stars spanning a small range of effective temperature, surface gravity, and metallicity and still obtain statistically mean- ingful samples. We intend to employ this strategy in our own analyses.

It is a pleasure to thank so many of our friends and colleagues for encouragement and advice throughout the course of this project, especially T. Beers and A. McWilliam. We appreciate 

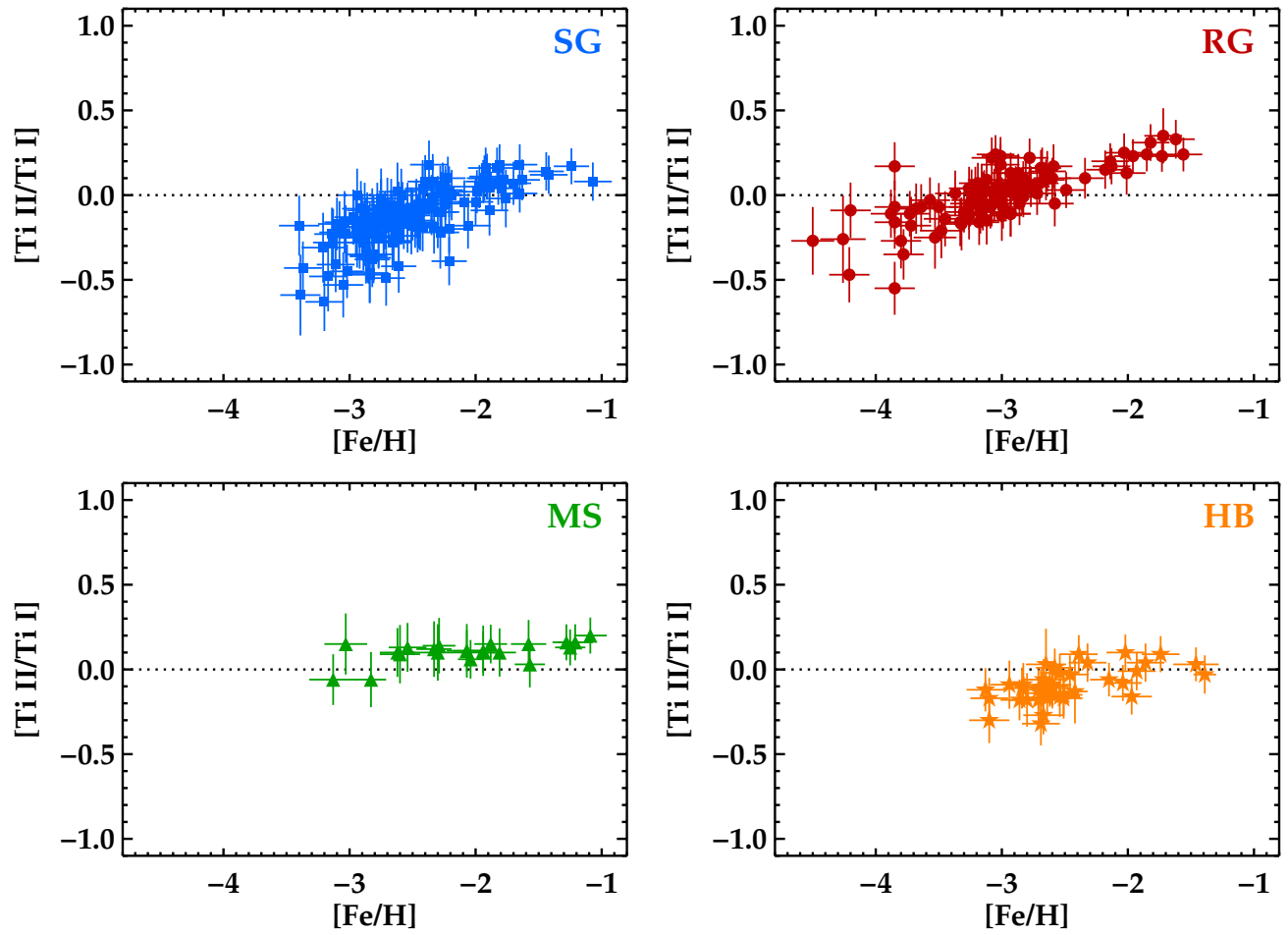

Figure 56. Ratios of the total titanium abundance derived from each of the ionized and neutral species as a function of [Fe/H]. Each star is displayed only if both species have been detected. Symbols are the same as in Figures 22 and 23.

(A color version of this figure is available in the online journal.)
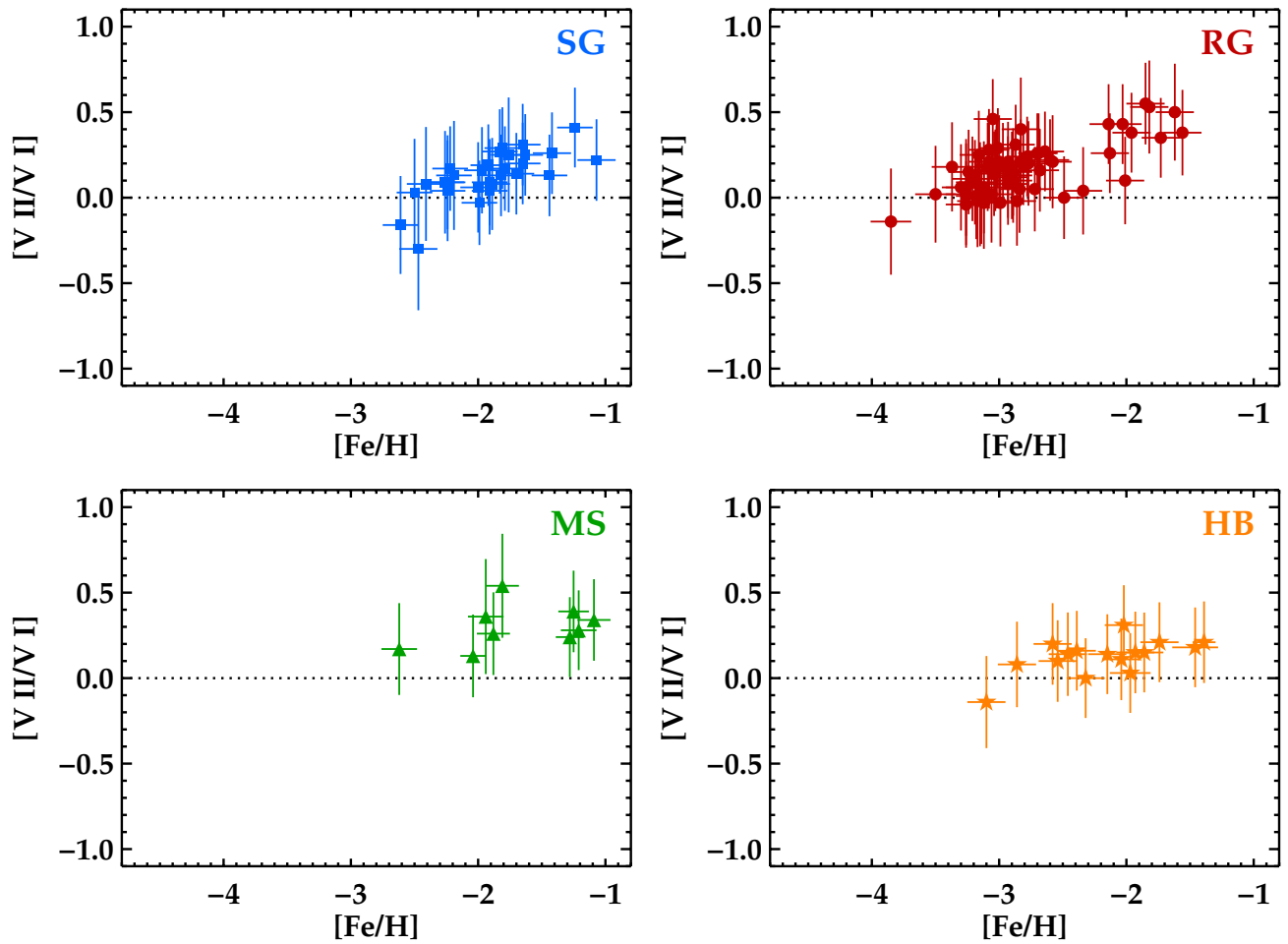

Figure 57. Ratios of the total vanadium abundance derived from each of the ionized and neutral species as a function of [Fe/H]. Each star is displayed only if both species have been detected. Symbols are the same as in Figures 22 and 23.

(A color version of this figure is available in the online journal.)

the patience of the Magellan and McDonald TAC members. I.U.R. also thanks D. Fabbian, K. Lind, A. McWilliam, and Y. Takeda for providing their interpolation codes, J. Lawler and M. Wood for sending results in advance of publication, J.
Sobeck for assistance with abundance comparisons and MOOG installations, the referees for their diligent readings of the manuscript, and $\mathrm{T}$. Beers for commenting on portions of an earlier version of the manuscript. 

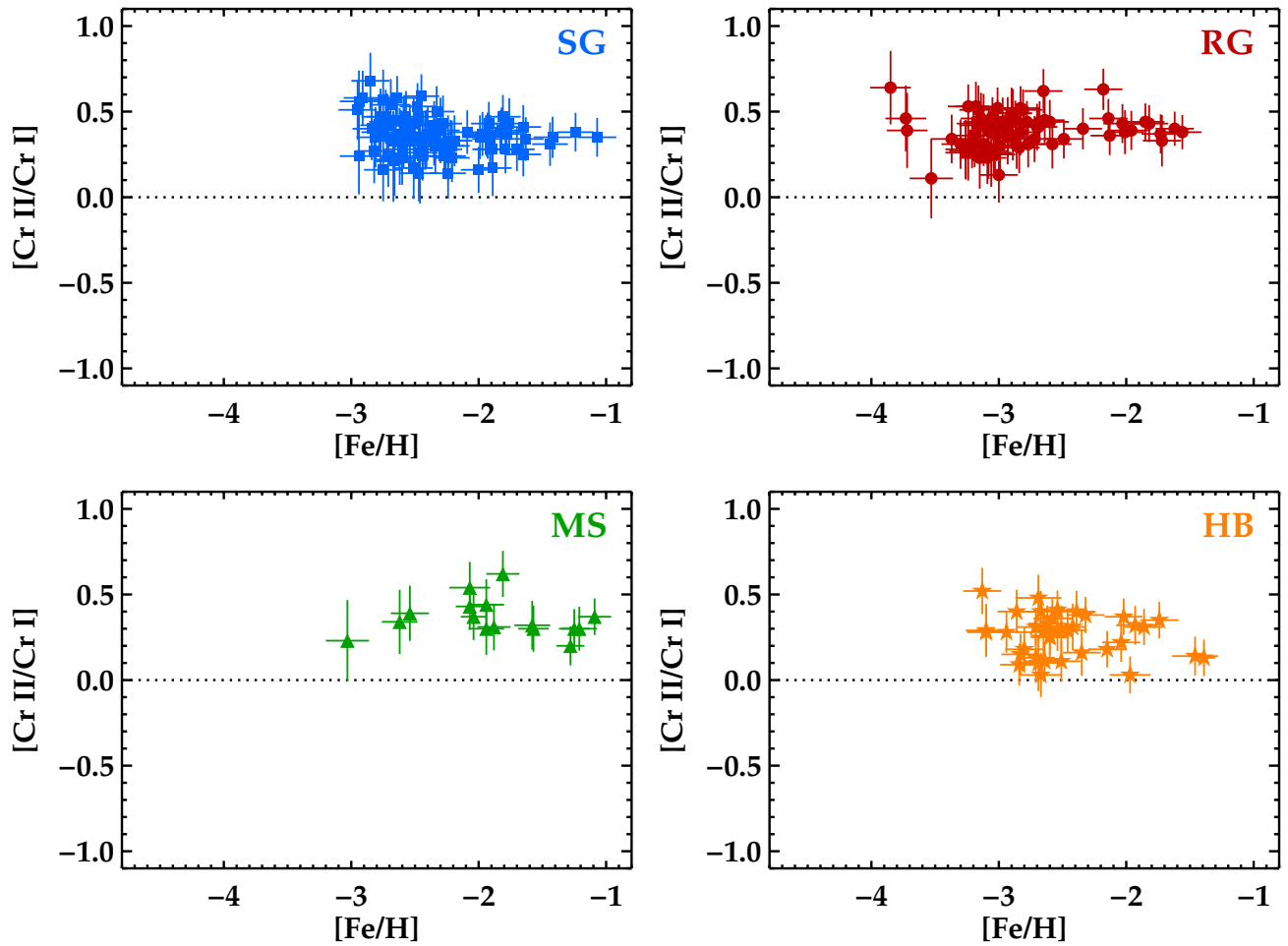

Figure 58. Ratios of the total chromium abundance derived from each of the ionized and neutral species as a function of [Fe/H]. Each star is displayed only if both species have been detected. Symbols are the same as in Figures 22 and 23.

(A color version of this figure is available in the online journal.)
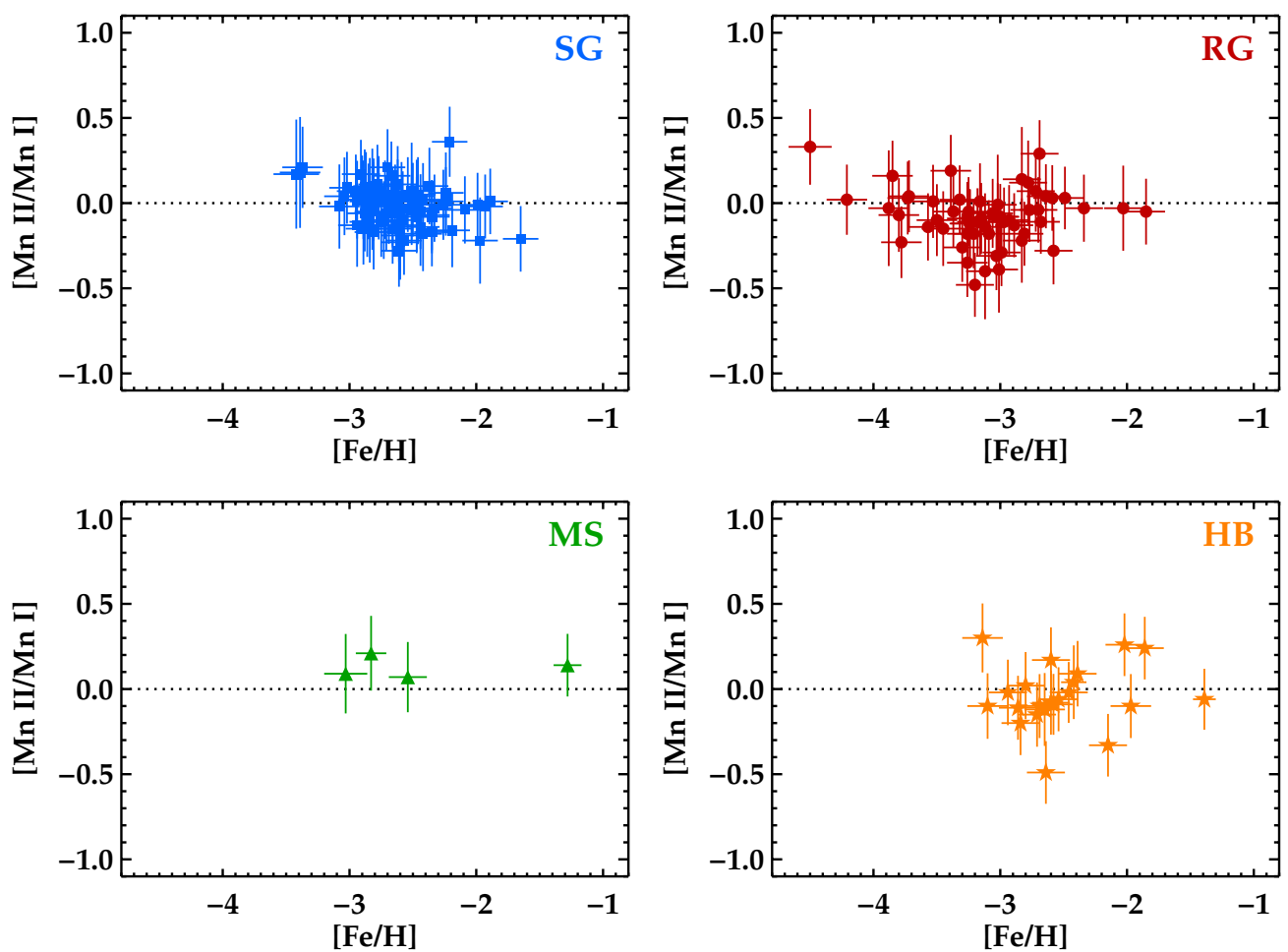

Figure 59. Ratios of the total manganese abundance derived from each of the ionized and neutral species as a function of [Fe/H]. Each star is displayed only if both species have been detected. Symbols are the same as in Figures 22 and 23.

(A color version of this figure is available in the online journal.)

This research has made use of NASA's Astrophysics Data System Bibliographic Services, the arXiv preprint server operated by Cornell University, the SIMBAD and VizieR databases hosted by the Strasbourg Astronomical Data Center, and the
Atomic Spectra Database hosted by the National Institute of Standards and Technology. Our appreciation for the reliability of these data archives cannot be understated. IRAF is distributed by the National Optical Astronomy Observatories, which are 
operated by the Association of Universities for Research in Astronomy, Inc., under cooperative agreement with the National Science Foundation. This publication makes use of data products from the Two Micron All Sky Survey, which is a joint project of the University of Massachusetts and the Infrared Processing and Analysis Center/California Institute of Technology, funded by the National Aeronautics and Space Administration and the National Science Foundation. The HET is a joint project of the University of Texas at Austin, the Pennsylvania State University, Stanford University, Ludwig-Maximilians-Universität München, and Georg-August-Universität Göttingen. The HET is named in honor of its principal benefactors, William P. Hobby and Robert E. Eberly.

I.U.R. acknowledges support by the Barbara McClintock Fellowship from the Carnegie Institution for Science. C.S. acknowledges support by the U.S. National Science Foundation (grants AST 06-07708, AST 09-08978, and AST 12-11585).

Facilities: HET (HRS), Magellan:Baade (MIKE), Magellan:Clay (MIKE), Smith (Tull)

\section{REFERENCES}

Aldenius, M., Lundberg, H., \& Blackwell-Whitehead, R. 2009, A\&A, 502, 989 Aldenius, M., Tanner, J. D., Johansson, S., Lundberg, H., \& Ryan, S. G. 2007, A\&A, 461, 767

Alonso, A., Arribas, S., \& Martínez-Roger, C. 1999a, A\&AS, 139, 335

Alonso, A., Arribas, S., \& Martínez-Roger, C. 1999b, A\&AS, 140, 261

Aoki, W., Barklem, P. S., Beers, T. C., et al. 2009, ApJ, 698, 1803

Aoki, W., Beers, T. C., Christlieb, N., et al. 2007, ApJ, 655, 492

Aoki, W., Beers, T. C., Lee, Y. S., et al. 2013, AJ, 145, 13

Aoki, W., Beers, T. C., Sivarani, T., et al. 2008, ApJ, 678, 1351

Aoki, W., Honda, S., Beers, T. C., et al. 2005, ApJ, 632, 611

Aoki, W., Norris, J. E., Ryan, S. G., Beers, T. C., \& Ando, H. 2002a, ApJ, 567,1166

Aoki, W., Norris, J. E., Ryan, S. G., Beers, T. C., \& Ando, H. 2002b, ApJL, 576, L141

Aoki, W., Ryan, S. G., Norris, J. E., et al. 2002c, ApJ, 580, 1149

Asplund, M. 2005, ARA\&A, 43, 481

Asplund, M., Grevesse, N., Sauval, A. J., \& Scott, P. 2009, ARA\&A, 47, 481

Babcock, H. D., \& Herzberg, L. 1948, ApJ, 108, 167

Barklem, P. S., \& Aspelund-Johansson, J. 2005, A\&A, 435, 373

Barklem, P. S., Christlieb, N., Beers, T. C., et al. 2005, A\&A, 439, 129

Barklem, P. S., Piskunov, N., \& O'Mara, B. J. 2000, A\&AS, 142, 467

Beers, T. C. 2013, in ASP Conf. Ser., CTIO 50th Anniversary Symposium (San Francisco, CA: ASP), in press

Beers, T. C., Chiba, M., Yoshii, Y., et al. 2000, AJ, 119, 2866

Beers, T. C., \& Christlieb, N. 2005, ARA\&A, 43, 531

Beers, T. C., Flynn, C., Rossi, S., et al. 2007, ApJS, 168, 128

Beers, T. C., Kage, J. A., Preston, G. W., \& Shectman, S. A. 1990, AJ, 100, 849

Beers, T. C., Preston, G. W., \& Shectman, S. A. 1985, AJ, 90, 2089

Beers, T. C., Preston, G. W., \& Shectman, S. A. 1992, AJ, 103, 1987 (BPS92)

Beers, T. C., \& Sommer-Larsen, J. 1995, ApJS, 96, 175

Bell, R. A., Eriksson, K., Gustafsson, B., \& Nordlund, A. 1976, A\&AS, 23, 37 Bernstein, R., Shectman, S. A., Gunnels, S. M., Mochnacki, S., \& Athey, A. E. 2003, Proc. SPIE, 4841, 1694

Bidelman, W. P., \& MacConnell, D. J. 1973, AJ, 78, 687

Biémont, É., Blagoev, K., Engström, L., et al. 2011, MNRAS, 414, 3350

Biémont, E., Garnir, H. P., Palmeri, P., Li, Z. S., \& Svanberg, S. 2000, MNRAS, 312,116

Biémont, E., Grevesse, N., Faires, L. M., Marsden, G., \& Lawler, J. E. 1989 , A\&A, 209, 391

Bohlin, R. C., Hill, J. K., Jenkins, E. B., et al. 1983, ApJS, 51, 277

Bond, H. E. 1970, ApJS, 22, 117

Bond, H. E. 1980, ApJS, 44, 517

Bonifacio, P., Centurion, M., \& Molaro, P. 1999, MNRAS, 309, 533

Bonifacio, P., Molaro, P., Beers, T. C., \& Vladilo, G. 1998, A\&A, 332, 672

Bonifacio, P., Monai, S., \& Beers, T. C. 2000, AJ, 120, 2065

Bonifacio, P., Sbordone, L., Caffau, E., et al. 2012, A\&A, 542, A87

Bonifacio, P., Spite, M., Cayrel, R., et al. 2009, A\&A, 501, 519

Bouwens, R. J., Illingworth, G. D., Franx, M., \& Ford, H. 2007, ApJ, 670, 928

Caffau, E., Bonifacio, P., François, P., et al. 2011, A\&A, 534, A4

Cardelli, J. A., Clayton, G. C., \& Mathis, J. S. 1989, ApJ, 345, 245
Cardon, B. L., Smith, P. L., Scalo, J. M., \& Testerman, L. 1982, ApJ, 260, 395 Carney, B. W. 1979, ApJ, 233, 211

Carney, B. W. 1983, AJ, 88, 610

Carney, B. W., \& Aaronson, M. 1979, AJ, 84, 867

Carney, B. W., \& Latham, D. W. 1986, AJ, 92, 60

Carney, B. W., Latham, D. W., Laird, J. B., \& Aguilar, L. A. 1994, AJ, 107,2240

Carney, B. W., Latham, D. W., Stefanik, R. P., \& Laird, J. B. 2008, AJ, 135, 196

Carney, B. W., Latham, D. W., Stefanik, R. P., Laird, J. B., \& Morse, J. A. 2003, AJ, 125, 293

Carretta, E., Gratton, R., Cohen, J. G., Beers, T. C., \& Christlieb, N. 2002, AJ, 124,481

Castelli, F., \& Kurucz, R. L. 2003, in IAU Symp. 210, Modelling of Stellar Atmospheres, ed. N. Piskunov et al. (Cambridge: Cambridge Univ. Press), A20

Cayrel, R., Depagne, E., Spite, M., et al. 2004, A\&A, 416, 1117

Cayrel, R., Hill, V., Beers, T. C., et al. 2001, Natur, 409, 691

Chang, T. N., \& Tang, X. 1990, JQSRT, 43, 207

Christlieb, N., Schörck, T., Frebel, A., et al. 2008, A\&A, 484, 721

Cohen, J. G., Christlieb, N., Beers, T. C., Gratton, R., \& Carretta, E. 2002, AJ, 124,470

Cohen, J. G., Christlieb, N., McWilliam, A., et al. 2004, ApJ, 612, 1107

Cohen, J. G., Christlieb, N., McWilliam, A., et al. 2008, ApJ, 672, 320

Cohen, J. G., McWilliam, A., Shectman, S., et al. 2006, AJ, 132, 137

Collet, R., Asplund, M., \& Trampedach, R. 2007, A\&A, 469, 687

Cooke, R., Pettini, M., Steidel, C. C., Rudie, G. C., \& Nissen, P. E. 2011, MNRAS, 417, 1534

Cowan, J. J., Sneden, C., Beers, T. C., et al. 2005, ApJ, 627, 238

Creevey, O. L., Thévenin, F., Boyajian, T. S., et al. 2012, A\&A, 545, A17

Demarque, P., Woo, J.-H., Kim, Y.-C., \& Yi, S. K. 2004, ApJS, 155, 667

Deng, L.-C., Newberg, H. J., Liu, C., et al. 2012, RAA, 12, 735

Den Hartog, E. A., Lawler, J. E., Sneden, C., \& Cowan, J. J. 2003, ApJS, 148,543

Den Hartog, E. A., Lawler, J. E., Sneden, C., \& Cowan, J. J. 2006, ApJS, 167,292

Den Hartog, E. A., Lawler, J. E., Sobeck, J. S., Sneden, C., \& Cowan, J. J. 2011, ApJS, 194, 35

Depagne, E., Hill, V., Spite, M., et al. 2002, A\&A, 390, 187

Doerr, A., Kock, M., Kwiatkowski, M., \& Werner, K. 1985, JQSRT, 33, 55

Dotter, A., Sarajedini, A., Anderson, J., et al. 2010, ApJ, 708, 698

Fabbian, D., Asplund, M., Barklem, P. S., Carlsson, M., \& Kiselman, D. 2009, A\&A, 500, 1221

Fitzpatrick, M. J., \& Sneden, C. 1987, BAAS, 19, 1129

François, P., Depagne, E., Hill, V., et al. 2007, A\&A, 476, 935

Frebel, A., Allende Prieto, C., Roederer, I. U., et al. 2008a, in ASP Conf. Ser. 393, New Horizons in Astronomy, ed. A. Frebel, J. R. Maund, J. Shen, \& M. H. Siegel (San Francisco, CA: ASP), 203

Frebel, A., Casey, A. R., Jacobson, H. R., \& Yu, Q. 2013, ApJ, 769, 57

Frebel, A., Collet, R., Eriksson, K., Christlieb, N., \& Aoki, W. 2008b, ApJ, 684,588

Fuhr, J. R., \& Wiese, W. L. 2009, in Atomic Transition Probabilities, CRC Handbook of Chemistry and Physics, ed. D. R. Lide (90th ed.; Boca Raton, FL: CRC), 10

Fulbright, J. P. 2000, AJ, 120, 1841

Fulbright, J. P. 2002, AJ, 123, 404

Fulbright, J. P., Wyse, R. F. G., Ruchti, G. R., et al. 2010, ApJL, 724, L104

García Pérez, A. E., Asplund, M., Primas, F., Nissen, P. E., \& Gustaffson, B. 2006, A\&A, 451, 621

Giclas, H. L., Burnham, R., \& Thomas, N. G. 1971, Lowell Proper Motion Survey Northern Hemisphere: The G Numbered Stars; 8991 Stars Fainter Than Magnitude 8 with Motions $>0$ '. 26/year (Flagstaff, AZ: Lowell Observatory) Giclas, H. L., Burnham, R., Jr., \& Thomas, N. G. 1978, LowOB, 8, 89

Gilroy, K. K., Sneden, C., Pilachowski, C. A., \& Cowan, J. J. 1988, ApJ, 327,298

Giridhar, S., Lambert, D. L., Gonzalez, G., \& Pandey, G. 2001, PASP, 113, 519 González Hernández, J. I., \& Bonifacio, P. 2009, A\&A, 497, 497

Gratton, R. G., Carretta, E., \& Castelli, F. 1996, A\&A, 314, 191

Gratton, R. G., Carretta, E., Desidera, S., et al. 2003, A\&A, 406, 131

Gratton, R. G., \& Sneden, C. 1988, A\&A, 204, 193

Gratton, R. G., \& Sneden, C. 1991, A\&A, 241, 501

Gratton, R. G., \& Sneden, C. 1994, A\&A, 287, 927

Griffin, R., \& Griffin, R. 1973, MNRAS, 162, 255

Gustafsson, B., Edvardsson, B., Eriksson, K., et al. 2008, A\&A, 486, 951

Harris, D. L., III, \& Upgren, A. R. 1964, ApJ, 140, 151

Hartmann, K., \& Gehren, T. 1988, A\&A, 199, 269

Hill, V., Plez, B., Cayrel, R., et al. 2002, A\&A, 387, 560 
Hinkle, K., Wallace, L., Valenti, J., \& Harmer, D. (ed.) 2000, Visible and Near Infrared Atlas of the Arcturus Spectrum 3727-9300 A (San Francisco, CA: ASP)

Hollek, J. K., Frebel, A., Roederer, I. U., et al. 2011, ApJ, 742, 54

Honda, S., Aoki, W., Ando, H., et al. 2004a, ApJS, 152, 113

Honda, S., Aoki, W., Kajino, T., et al. 2004b, ApJ, 607, 474

Ishigaki, M., Chiba, M., \& Aoki, W. 2010, PASJ, 62, 143

Ito, H., Aoki, W., Beers, T. C., et al. 2013, ApJ, 773, 33

Ivans, I. I., Simmerer, J., Sneden, C., et al. 2006, ApJ, 645, 613

Ivans, I. I., Sneden, C., Gallino, R., Cowan, J. J., \& Preston, G. W. 2005, ApJL, 627, L145

Ivans, I. I., Sneden, C., James, C. R., et al. 2003, ApJ, 592, 906

Ivarsson, S., Andersen, J., Nordström, B., et al. 2003, A\&A, 409, 1141

Johnson, J. A. 2002, ApJS, 139, 219

Johnson, J. A., Herwig, F., Beers, T. C., \& Christlieb, N. 2007, ApJ, 658, 1203

Keller, S. C., SkyMapper Team, \& Aegis Team. 2012, in ASP Conf. Ser. 458, Galactic Archaeology: Near-Field Cosmology and the Formation of the Milky Way, ed. W. Aoki, M. Ishigaki, T. Suda, T. Tsujimoto, \& N. Arimoto (San Francisco, CA: ASP), 409

Kelson, D. D. 2003, PASP, 115, 688

Kiselman, D. 2001, NewAR, 45, 559

Kurucz, R. L., \& Bell, B. 1995, Kurucz CD-ROM (Cambridge, MA: Smithsonian Astrophysical Observatory)

Lai, D. K., Bolte, M., Johnson, J. A., \& Lucatello, S. 2004, AJ, 128, 2402

Lai, D. K., Bolte, M., Johnson, J. A., et al. 2008, ApJ, 681, 1524

Latham, D. W., Davis, R. J., Stefanik, R. P., Mazeh, T., \& Abt, H. A. 1991, AJ, 101,625

Latham, D. W., Stefanik, R. P., Torres, G., et al. 2002, AJ, 124, 1144

Lawler, J. E., Bonvallet, G., \& Sneden, C. 2001a, ApJ, 556, 452

Lawler, J. E., \& Dakin, J. T. 1989, JOSAB, 6, 1457

Lawler, J. E., Den Hartog, E. A., Labby, Z. E., et al. 2007, ApJS, 169, 120

Lawler, J. E., Den Hartog, E. A., Sneden, C., \& Cowan, J. J. 2006, ApJS, 162,227

Lawler, J. E., Guzman, A., Wood, M. P., Sneden, C., \& Cowan, J. J. 2013, ApJS, 205,11

Lawler, J. E., Sneden, C., \& Cowan, J. J. 2004, ApJ, 604, 850

Lawler, J. E., Sneden, C., Cowan, J. J., Ivans, I. I., \& Den Hartog, E. A. 2009, ApJS, 182, 51

Lawler, J. E., Sneden, C., Cowan, J. J., et al. 2008, ApJS, 178, 71

Lawler, J. E., Wickliffe, M. E., Cowley, C. R., \& Sneden, C. 2001b, ApJS, 137,341

Lawler, J. E., Wickliffe, M. E., den Hartog, E. A., \& Sneden, C. 2001c, ApJ, 563,1075

Lawler, J. E., Wyart, J.-F., \& Blaise, J. 2001d, ApJS, 137, 351

Lazauskaite, R., \& Tautvaisiene, G. 1990, VilOB, 85, 30

Li, R., Chatelain, R., Holt, R. A., et al. 2007, PhyS, 76, 577

Lind, K., Asplund, M., \& Barklem, P. S. 2009, A\&A, 503, 541

Lind, K., Asplund, M., Barklem, P. S., \& Belyaev, A. K. 2011, A\&A, 528, A103

Lind, K., Bergemann, M., \& Asplund, M. 2012, MNRAS, 427, 50

Ljung, G., Nilsson, H., Asplund, M., \& Johansson, S. 2006, A\&A, 456, 1181

Lucatello, S., Tsangarides, S., Beers, T. C., et al. 2005, ApJ, 625, 825

Luck, R. E., \& Bond, H. E. 1981, ApJ, 244, 919

Luck, R. E., \& Bond, H. E. 1985, ApJ, 292, 559

Luyten, W. J. 1979 , NLTT Catalogue, Volume I: +90 to +30 , Volume II: +30 to 0 (Minneapolis, MN: Univ. Minnesota)

Magain, P. 1984, A\&A, 134, 189

Magain, P. 1989, A\&A, 209, 211

Masseron, T., Johnson, J. A., Lucatello, S., et al. 2012, ApJ, 751, 14

Masseron, T., Johnson, J. A., Plez, B., et al. 2010, A\&A, 509, A93

Matejek, M. S., \& Simcoe, R. A. 2012, ApJ, 761, 112

McWilliam, A. 1998, AJ, 115, 1640

McWilliam, A., Preston, G. W., Sneden, C., \& Searle, L. 1995a, AJ, 109, 2757

McWilliam, A., Preston, G. W., Sneden, C., \& Shectman, S. 1995b, AJ, 109,2736

Meléndez, J., \& Barbuy, B. 2009, A\&A, 497, 611

Molaro, P., \& Bonifacio, P. 1990, A\&A, 236, L5

Molaro, P., \& Castelli, F. 1990, A\&A, 228, 426

Mucciarelli, A. 2011, A\&A, 58, A44

Nicolet, B. 1978, A\&AS, 34, 1

Nilsson, H., Hartman, H., Engström, L., et al. 2010, A\&A, 511, A16

Nilsson, H., Ljung, G., Lundberg, H., \& Nielsen, K. E. 2006, A\&A, 445, 1165

Nilsson, H., Zhang, Z. G., Lundberg, H., Johansson, S., \& Nordström, B. 2002, A\&A, 382, 368

Nitz, D. E., Kunau, A. E., Wilson, K. L., \& Lentz, L. R. 1999, ApJS, 122, 557

Nordström, B., et al. 2004, A\&A, 418, 989

Norris, J. 1986, ApJS, 61, 667

Norris, J., Bessell, M. S., \& Pickles, A. J. 1985, ApJS, 58, 463
Norris, J. E., Bessell, M. S., Yong, D., et al. 2013, ApJ, 762, 25

Norris, J. E., Peterson, R. C., \& Beers, T. C. 1993, ApJ, 415, 797

Norris, J. E., Ryan, S. G., \& Beers, T. C. 1996, ApJS, 107, 391

Norris, J. E., Ryan, S. G., \& Beers, T. C. 1997, ApJL, 489, L169

Norris, J. E., Ryan, S. G., \& Beers, T. C. 1999, ApJS, 123, 639

Norris, J. E., Ryan, S. G., \& Beers, T. C. 2001, ApJ, 561, 1034

O'Brian, T. R., Wickliffe, M. E., Lawler, J. E., Whaling, W., \& Brault, J. W. 1991, JOSAB, 8, 1185

Palmeri, P., Fischer, C. F., Wyart, J.-F., \& Godefroid, M. R. 2005, MNRAS, 363,452

Perryman, M. A. C., \& ESA, 1997, in The Hipparcos and Tycho Catalogues (ESA Special Publication Series, Vol. 1200; Noordwijk: ESA)

Peterson, R. C., Kurucz, R. L., \& Carney, B. W. 1990, ApJ, 350, 173

Pickering, J. C., Thorne, A. P., \& Perez, R. 2001, ApJS, 132, 403

Pickering, J. C., Thorne, A. P., \& Perez, R. 2002, ApJS, 138, 247

Piskunov, N. E., \& Valenti, J. A. 2002, A\&A, 385, 1095

Pourbaix, D., Tokovinin, A. A., Batten, A. H., et al. 2004, A\&A, 424, 727

Preston, G. W. 2009, PASA, 26, 372

Preston, G. W., Beers, T. C., \& Shectman, S. A. 1994, AJ, 108, 538

Preston, G. W., Shectman, S. A., \& Beers, T. C. 1991, ApJS, 76, 1001

Preston, G. W., \& Sneden, C. 2000, AJ, 120, 1014

Preston, G. W., \& Sneden, C. 2001, AJ, 122, 1545

Preston, G. W., Sneden, C., Thompson, I. B., Shectman, S. A., \& Burley, G. S. 2006a, AJ, 132, 85

Preston, G. W., Thompson, I. B., Sneden, C., Stachowski, G., \& Shectman, S. A. 2006 b, AJ, 132,1714

Primas, F., Molaro, P., \& Castelli, F. 1994, A\&A, 290, 885

Ramírez, I., \& Meléndez, J. 2005, ApJ, 626, 446

Ramsey, L. W., Adams, M. T., Barnes, T. G., et al. 1998, Proc. SPIE, 3352, 34 Rhee, J. 2001, PASP, 113, 1569

Rockosi, C. M. 2012, BAAS, 219, 205.01

Roederer, I. U., Frebel, A., Shetrone, M. D., et al. 2008a, ApJ, 679, 1549

Roederer, I. U., Kratz, K.-L., Frebel, A., et al. 2009, ApJ, 698, 1963

Roederer, I. U., \& Lawler, J. E. 2012, ApJ, 750, 76

Roederer, I. U., Lawler, J. E., Sneden, C., et al. 2008b, ApJ, 675, 723

Roederer, I. U., Lawler, J. E., Sobeck, J. S., et al. 2012, ApJS, 203, 27

Roederer, I. U., Preston, G. W., Thompson, I. B., Shectman, S. A., \& Sneden, C. 2014, ApJ, 784, 158

Roederer, I. U., Sneden, C., Thompson, I. B., Preston, G. W., \& Shectman, S. A. 2010, ApJ, 711, 573

Rossi, S., Beers, T. C., Sneden, C., et al. 2005, AJ, 130, 2804

Ryan, S. G. 1989, AJ, 98, 1693

Ryan, S. G., \& Norris, J. E. 1991, AJ, 101, 1835

Ryan, S. G., Norris, J. E., \& Beers, T. C. 1996, ApJ, 471, 254

Ryan, S. G., Norris, J. E., \& Bessell, M. S. 1991, AJ, 102, 303

Saha, M. N. 1921, RSPSA, 99, 135

Sandage, A. 1969, ApJ, 158, 1115

Sandage, A., \& Kowal, C. 1986, AJ, 91, 1140

Sargent, W. L. W., Boksenberg, A., \& Steidel, C. C. 1988, ApJS, 68, 539

Sbordone, L., Bonifacio, P., Caffau, E., et al. 2010, A\&A, 522, A26

Schlegel, D. J., Finkbeiner, D. P., \& Davis, M. 1998, ApJ, 500, 525

Schuster, W. J., Beers, T. C., Michel, R., Nissen, P. E., \& García, G. 2004, A\&A, 422, 527

Shetrone, M., Cornell, M. E., Fowler, J. R., et al. 2007, PASP, 119, 556

Shi, J. R., Gehren, T., Mashonkina, L., \& Zhao, G. 2009, A\&A, 503, 533

Simmerer, J., Sneden, C., Cowan, J. J., et al. 2004, ApJ, 617, 1091

Sivarani, T., Beers, T. C., Bonifacio, P., et al. 2006, A\&A, 459, 125

Skrutskie, M. F., Cutri, R. M., Stiening, R., et al. 2006, AJ, 131, 1163

Smith, V. V., Lambert, D. L., \& Nissen, P. E. 1998, ApJ, 506, 405

Sneden, C., Cowan, J. J., \& Gallino, R. 2008, ARA\&A, 46, 241

Sneden, C., Cowan, J. J., Lawler, J. E., et al. 2003, ApJ, 591, 936

Sneden, C., Lawler, J. E., Cowan, J. J., Ivans, I. I., \& Den Hartog, E. A. 2009, ApJS, 182, 80

Sneden, C., McWilliam, A., Preston, G. W., et al. 1996, ApJ, 467, 819

Sneden, C., Preston, G. W., \& Cowan, J. J. 2003, ApJ, 592, 504

Sneden, C., Preston, G. W., McWilliam, A., \& Searle, L. 1994, ApJL, 431, L27

Sneden, C. A. 1973, PhD thesis, Univ. Texas at Austin

Sobeck, J. S., Kraft, R. P., Sneden, C., et al. 2011, AJ, 141, 175

Sobeck, J. S., Lawler, J. E., \& Sneden, C. 2007, ApJ, 667, 1267

Spite, M., Cayrel, R., Plez, B., et al. 2005, A\&A, 430, 655

Spite, M., Depagne, E., Nordström, B., et al. 2000, A\&A, 360, 1077

Stephens, A., \& Boesgaard, A. M. 2002, AJ, 123, 1647

Takeda, Y., Zhao, G., Chen, Y.-Q., Qiu, H.-M., \& Takada-Hidai, M. 2002, PASJ, 54,275

Thévenin, F., \& Idiart, T. P. 1999, ApJ, 521, 753

Thompson, I. B., Ivans, I. I., Bisterzo, S., et al. 2008, ApJ, 677, 556

Tsangarides, S., Ryan, S. G., \& Beers, T. C. 2004, MmSAI, 75, 772 
Tull, R. G. 1998, Proc. SPIE, 3355, 387

Tull, R. G., MacQueen, P. J., Sneden, C., \& Lambert, D. L. 1995, PASP, 107,251

Unsöld, A. 1955, Physik der Sternatmosphären (Berlin: Springer)

Van Eck, S., Goriely, S., Jorissen, A., \& Plez, B. 2003, A\&A, 404, 291

van Leeuwen, F. 2007, A\&A, 474, 653

Whaling, W., \& Brault, J. W. 1988, PhyS, 38, 707

Wickliffe, M. E., \& Lawler, J. E. 1997, JOSAB, 14, 737
Wickliffe, M. E., Lawler, J. E., \& Nave, G. 2000, JQSRT, 66, 363

Wickliffe, M. E., Salih, S., \& Lawler, J. E. 1994, JQSRT, 51, 545

Wisotzki, L., Christlieb, N., Bade, N., et al. 2000, A\&A, 358, 77

Wood, M. P., Lawler, J. E., Sneden, C., \& Cowan, J. J. 2013, ApJS, 208, 27

Yanny, B., Rockosi, C., Newberg, H. J., et al. 2009, AJ, 137, 4377

Yong, D., Norris, J. E., Bessell, M. S., et al. 2013, ApJ, 762, 26

Zhang, L., Ishigaki, M., Aoki, W., Zhao, G., \& Chiba, M. 2009, ApJ, 706, 1095

Zhao, G., \& Magain, P. 1990, A\&A, 238, 242 